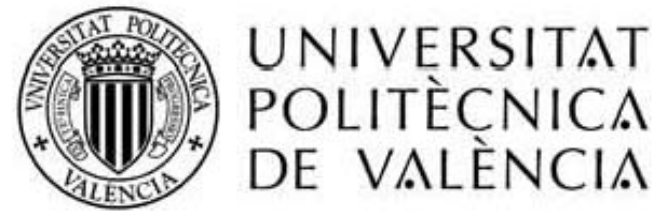

Departamento de Biotecnología

Aplicación de los estudios de resultados de salud en el hospital

Francisco Javier Tomás Carrera Hueso

TESIS DOCTORAL

Director:

Profesor D. Manuel Hernández Pérez 

Manuel Hernández Pérez, Profesor Titular del Departamento de Biotecnología de la Universidad Politécnica de Valencia,

\section{CERTIFICA}

Que la presente memoria titulada "Aplicación de los estudios de resultados de salud en el hospital" ha sido realizada por Francisco Javier Tomás Carrera Hueso bajo su dirección en el Departamento de Biotecnología de la U.P.V. y constituye su Memoria de Tesis para optar al grado de Doctor por la Universidad Politécnica de Valencia.

Y para que así conste a todos los efectos oportunos, firma el presente certificado en Valencia, a catorce de abril de dos mil once.

Manuel Hernández Pérez 

Ituxi, Leire y Losé

a mis padres 



\section{AGRADECIMIENTDS}

Quisiera hacer mi público agradecimiento a mi familia, a los que dedico este trabajo, que soportan muchas horas de mi ausencia debido a mis compromisos laborales. Ellos son mi motor y objetivo de felicidad, ya que ésta sin ser compartida no sería lo mismo.

A mis padres, José (d.e.p) y Josefina, que supieron implantar en mi la suficiente cordura, sentido común, inteligencia, educación y todos los demás buenos valores, desde su ejemplo diario y esforzado que supone sacar adelante una familia numerosa y con el éxito de que todos seamos profesionales universitarios.

A mis hermanos, Luisa, Pepa, José, Susa, Migue y Alicia; por su desinteresada ayuda y amor que siempre me han mostrado, siendo todos ellos un ineludible referente en todas y cada una de las situaciones que el devenir de la vida me hizo transitar.

A mis hermanos políticos: Nacho, José, Joaquín, Marga y Fernando; por servirme de apoyo y comprender mi situación, y sobre todo por vuestro desinteresado afecto y amor conmigo y los míos. Siempre os he considerado como hermanos y así me lo habéis demostrado.

A mis sobrinos: Luisa, Candela, Belén, Marcos, Emma, Andrés, Ana, María, Marta, Gonzalo, Fernando y Javier; por permitirme quereros como hijos o hermanos y aprender también de vosotros las dificultosas relaciones personales.

A mi tía Elvira, por su desinteresada ayuda y bondad, y por estar siempre ahí.

A mis amigos de toda la vida: Manuel, Alberto, Manolo, Jaime, Carlos, David, Jorge, y todos los demás que no cabéis en estas líneas; por oírme, comprenderme, animarme y en definitiva ayudarme cuando os necesité.

A la Universidad Politécnica de Valencia y concretamente al Departamento de Biotecnología que por medio del Profesor Manuel Hernández me ha brindado la oportunidad de realizar este trabajo para obtener el título de Doctor. 
A mis compañeros, que de ellos aprendí todo lo que soy profesionalmente hoy en día. Especialmente a los del Servicio de Farmacia del Hospital Dr Moliner donde trabajo actualmente y a los del Servicio de Farmacia del Hospital General de Lanzarote. También he de ser agradecido con todos mis compañeros médicos por sus valiosas enseñanzas.

A los pacientes, que ellos son mi fuente de enseñanza para saber lo que realmente es el sentido de la vida.

Por último, aunque no pueda recoger aquí a todos aquellos que me enseñaron, animaron y estimularon en mi trayectoria personal, mi más sentido agradecimiento por la oportunidad que me dieron de mejorar, aprender y crecer, no solo profesionalmente sino más importante como persona.

Para finalizar quisiera nombrar uno de los principios que me han guiado en mi vida y no es otro que:

"Sé el más exigente aprendiendo y el más generoso al enseñar o compartir el conocimiento" 


\section{Índice}

RESUMEN............................................................................

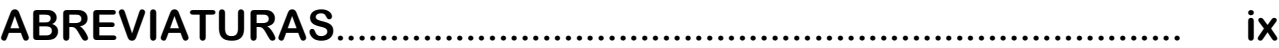

INTRODUCCIÓN.................................................................... 1

1.1. Sostenibilidad del sistema sanitario........................ 2

1.2. Gestión clínica y atención farmacéutica..................... 8

1.3. Estudios de resultados sanitarios............................. 12

1.3.1. Eficacia, efectividad y eficiencia...................................... 14

1.4. Estudios farmacoeconómicos................................... 16

1.4.1. Diseño, alternativas, perspectiva y horizonte temporal...... 17

1.4.2. Costes...................................................................... 20

1.4.3. Beneficios................................................................... 23

1.4.4. Tipos de evaluaciones sanitarias..................................... 25

1.4.5. Modelización.................................................................. 31

1.4.6. Análisis de la incertidumbre .......................................... 32

1.5. Estudios de calidad de vida, CVRS........................... 38

1.5.1. Cuestionarios genéricos............................................... 40

1.5.2. Cuestionarios específicos.............................................. 42

1.5.3. Cuestionarios para aspectos específicos de CVRS............ 43

OBJETIVOS ............................................................................ 44

RESULTADOS Y DISCUSIÓN.................................................. 46 
Capítulo 1: Carrera-Hueso FJ. Análisis de los resultados sanitarios y su aplicación. Rev Med Univ Navarra 1997; 41: 31-38.

Capítulo 2: Carrera-Hueso FJ. Aplicación de los estudios 56 farmacoeconómicos en el hospital. Med Clín (Barc) 1998; 111: 347-53.

Capítulo 3: Carrera-Hueso FJ. Estudios de calidad de vida relacionados con la salud. Farm Clín 1997; 14: 98-109.

Capítulo 4: Carrera-Hueso FJ. Calidad de vida en un paciente epiléptico. Farm Clín 1997; 14: 74-89.

Capítulo 5: Carrera-Hueso FJ, Ramón Barrios A, Carrera Hueso JA, Poquet Jornet JE. Coste-efectividad de la tromboprofilaxis extendida en fractura de cadera: fondaparinux frente a enoxaparina. Aten Farm 2010; 12 (3): 144-55.

Capítulo 6: Carrera-Hueso FJ, Poquet Jornet JE, Ramón Barrios A, Conde Fernández F. Coste-efectividad en la inducción del parto con dinoprostona. Aten Farm 2010; 12 (6): 359-68.

Capítulo 7: Carrera-Hueso FJ, Ramón Barrios A. Análisis de sensibilidad estructural. Farm Hosp 2011; 35 (Supl 1): 10-17.

Capítulo 8: Amat Díaz M, Poveda Andrés JL, Carrera-Hueso FJ. Aplicación de la farmacoeconomía en la gestión clínica. Farm Hosp 2011; 35 (Supl 1): 18-24.

Capítulo 9: Poquet Jornet JE, Carrera-Hueso FJ, Gasent Blesa JM, Peris Godoy M. Aspectos farmacoeconómicos de los citostáticos orales. Farm Hosp 2011; 35 (Supl 1): 25-31.

DISCUSIÓN GENERAL

CONCLUSIONES 
RESUMEN 


\section{Resumen}

En el presente trabajo se pretende mostrar la incorporación de los estudios farmacoeconómicos en el ámbito hospitalario, aplicar los estudios farmacoeconómicos en situaciones clínicas reales adaptados a nuestro entorno, utilizar el análisis de la incertidumbre para garantizar la robustez de los resultados obtenidos, realizar un análisis de sensibilidad estructural, mostrar la utilidad de medir la calidad de vida relacionada con la salud en la atención sanitaria a los pacientes y utilizar la información proporcionada por los estudios de resultados en salud tanto como herramienta en la toma de decisiones farmacoterapéuticas como para la justificación de servicios clínicos.

Se exponen en nueve capítulos los estudios publicados y posteriormente se discuten de manera general los mismos según las principales estrategias para su incorporación en el hospital, como son: a) utilizar la bibliografía publicada, b) mediante realización de modelos, c) realizando un ensayo clínico, un estudio pragmático y/o un estudio observacional.

Se concluye que es factible aplicar los estudios de resultados sanitarios en la práctica hospitalaria diaria y su traslación permite tomar mejores decisiones clínicas. Se deben utilizar todas las opciones que ofrecen los modelos farmacoeconómicos y el análisis de sensibilidad, ya sea determinístico o estocástico, dado que es una herramienta necesaria para verificar la robustez de sus resultados. El análisis de sensibilidad estructural aporta información valiosa sobre el problema que se quiere representar y da una mayor validez y fiabilidad al modelo. La calidad de vida relacionada con la salud es un resultado en sí mismo por lo que debemos incorporarla como variable en nuestros estudios. Los programas asistenciales de atención farmacéutica y nuevos servicios clínicos se verán reforzados al aplicar esta tecnología en su implantación para demostrar su valor. 


\section{Resum}

En el present treball es pretén mostrar la incorporació dels estudis farmacoeconòmics en l'àmbit hospitalari, aplicar els estudis farmacoeconòmics adaptats al nostre entorn en situacions clíniques reals, utilitzar l'anàlisi de la incertesa per garantir la robustesa dels resultats obtinguts, realitzar una anàlisi de sensibilitat estructural, mostrar la utilitat de mesurar la qualitat de vida relacionada amb la salut en l'atenció sanitària als pacients, i utilitzar la informació proporcionada pels estudis de resultats en salut tant com a eina en la presa de decisions farmacoterapèutiques com per a la justificació de serveis clínics.

S'exposen en nou capítols els estudis publicats i posteriorment es discuteixen de manera general els mateixos, segons les principals estratègies per a incorporar-los a I'hospital, com són: a) utilitzar la bibliografia publicada, b) mitjançant la realització de models, c) realitzant un assaig clínic, estudi pragmàtic y/o un estudi observacional.

Es conclou que és factible aplicar els estudis de resultats sanitaris a la pràctica hospitalària diària, i la seva translació permet prendre millors decisions clíniques. S'han d'utilitzar totes les opcions que ofereixen els models farmacoeconòmics i l'anàlisi de sensibilitat, ja sigui determinístic o estocàstic, atès que és una eina necessària per verificar la robustesa dels seus resultats. L'anàlisi de sensibilitat estructural aporta informació valuosa sobre el problema que es vol representar i dóna una major validesa i fiabilitat al model. La qualitat de vida relacionada amb la salut és un resultat en si mateix per la qual cosa l'hem d'incorporar com a variable als nostres estudis. Els programes assistencials d'atenció farmacèutica i els nous serveis clínics es veuran reforçats en aplicar aquesta tecnologia en la seva implantació per a demostrar el seu valor. 


\section{Summary}

The presented work shows the incorporation of pharmacoeconomic studies in the hospital, implementing pharmacoeconomic studies in current clinical situations adapted to our environment, using analysis of uncertainties, like analysis of structural sensitivity, shows to ensure the robustness of the final results. It Shows too the usefulness of measuring the quality of life related to the health care of patients. The information provided by health studies influences on the pharmacotherapeutic decision-making and in justifying clinical services.

It is presented in nine chapters which include published studies with general discussions on the main strategies for the incorporation of pharmacoeconomics into the hospital, such as: a) use the published literature, b) by construction of models, c) performing a clinical trial or pragmatic or observational study.

It concludes that it is feasible to apply the outcomes of health studies in the everyday hospital practice in order to make better clinical decisions. All the options offered by pharmacoeconomic models and sensitivity analysis must be used, either deterministic or stochastic, since they are considered as a necessary tool to verify the robustness of their results. Structural sensitivity analysis provides valuable information about the problem being represented, and increase the validity and reliability of the model. The quality of life related to health is a result in itself and we must incorporate it as a variable in our studies. Assistance programs of pharmaceutical care and new clinical services will be enhanced by applying the implementation of this technology to demonstrate its value. 


\section{Abreviaturas}

AGEMED: $\quad$ Agencia Española del Medicamento.

AVAC: $\quad$ Años de vida ajustados por calidad.

CFT: $\quad$ Comisión de Farmacia y Terapéutica.

CVRS: $\quad$ Calidad de vida relacionada con la salud.

D.D.D.: $\quad$ Dosis Diarias Definidas.

EuroQol Cuestionario de calidad de vida EuroQol.

FE: $\quad$ Farmacoeconomía.

GENESIS: Grupo de Evaluación de Novedades, Estandarización e Investigación en Selección de Medicamentos.

GRD: $\quad$ Grupos relacionados con el diagnóstico.

MSPSI: Ministerio de Sanidad, Política Social e Igualdad.

NICE Instituto Británico para la Excelencia Clínica.

NHP Cuestionario de calidad de vida Nottingham Health Profile.

OCDE: $\quad$ Organización para la Cooperación y el Desarrollo Económicos.

OMS: $\quad$ Organización Mundial de la Salud.

PIB: $\quad$ Producto Interior Bruto.

PVL: $\quad$ Precio de Venta de Laboratorio.

RNM: $\quad$ Resultados negativos asociados a la medicación.

SEFH: $\quad$ Sociedad Española de Farmacia Hospitalaria.

SIP Cuestionario calidad de vida Sickness Impact Profile.

SF: $\quad$ Servicio de Farmacia.

SF-36: $\quad$ Cuestionario de calidad de vida Medical Outcomes Study Short Form-36.

SFT: $\quad$ Seguimiento Farmacoterapéutico.

SNS: $\quad$ Sistema Nacional de Salud. 


\section{INTRODUCCIÓN}




\subsection{Sostenibilidad del sistema sanitario.}

El derecho a la protección de su salud se recoge en nuestra Carta Magna y mediante la Ley 14/1986 General de Sanidad, y se define el SNS como un instrumento esencial del Estado de Bienestar, que se rige por los principios básicos de unidad, universalidad, equidad y solidaridad, y contribuye efectivamente a la cohesión económica y social de nuestro país. El apoyo a la creatividad y la innovación en los servicios sanitarios es parte de la misión del propio SNS.

Asimismo, durante el transcurso de los años, el SNS ha sabido plasmar su capacidad para proporcionar resultados óptimos en relación a distintos parámetros referentes al estado de salud de la población. Otros resultados de salud son atribuibles a la acción del propio sistema sanitario como parámetros de cobertura, acceso y equidad financiera, resultados relacionados con la calidad y seguridad de la atención sanitaria, así como, en el grado de satisfacción de los usuarios y su legitimación por parte de la población (García-Armesto et al., 2010). Y todo ello, aparentemente, a un "buen precio", traducido con un porcentaje del PIB dedicado a sufragar el gasto sanitario por debajo del promedio europeo y situado en un $9 \%$ para el 2008, según los últimos datos proporcionados por la OCDE (OCDE, 2010).

Pero sería equívoco caer en la euforia momentánea que puede desprenderse de lo logros expuestos. Así, diferentes factores como el envejecimiento de la población, la mayor complejidad de los problemas de salud, el incremento sustancial de sus necesidades y exigencias socio-sanitarias, la inmigración, el aluvión constante de nuevas tecnologías e incluso la amplia variabilidad en la práctica médica entre muchos otros factores, pueden hacer tambalear a cualquier modelo sanitario. Un sistema sanitario que, además, se soporta en base a las recaudaciones fiscales y, por ende, está confinado a los límites de las contribuciones. Simplificando, en un escenario en el que los recursos son limitados y unido a situaciones específicas, como son los fenómenos sociales o incluso la actual crisis económica, el debate acerca de la sostenibilidad de nuestro sistema sanitario se acrecienta y están seguramente en la agenda de las decisiones políticas futuras a corto plazo. 
El gasto sanitario total en España en 2007 ascendió a 88.828 millones de $€$, del cual un $71,8 \%$ (63.768 millones $€$ ) correspondió al gasto Sanitario público, incluyendo gasto de cuidados de larga duración. Como porcentaje del PIB, este gasto sanitario total representó un $8,40 \%$ y el gasto farmacéutico un $1,88 \%$. Desde una dimensión funcional del gasto sanitario público, los servicios hospitalarios y especializados $(54,0 \%)$ son los que representan un mayor porcentaje del mismo, seguidos por la prestación farmacéutica, 21,70\% y los servicios de Atención Primaria de salud con un $15,7 \%$. Los servicios de Salud Pública con $1,4 \%$ del gasto, muestran un aparentemente pequeño peso relativo consecuencia de, además de la manera de definir y clasificar esta actividad en los sistemas contables, por el hecho de que las actividades de salud pública, prevención y promoción, se desarrollan básicamente a través de la red de Atención Primaria y no están contabilizadas de manera específica (Martín-Conde et al., 2011).

La crisis económica y financiera, que manifestó sus primeros efectos en Europa durante la segunda mitad de 2007, impactó con especial crudeza en 2009. En ese ejercicio, el PIB de la UE-27 registró una caída real del $-4,2 \%$, en comparación con el crecimiento del $+0,8 \%$ del ejercicio anterior. Por ello, la práctica totalidad de ámbitos de la Administración Pública ha ejecutado una serie de medidas que afectarán a la inversión pública en infraestructuras, a la masa salarial de los empleados públicos, a la revalorización de las pensiones, y a otras partidas de gasto público entre las que se encuentran dos medidas de reducción del gasto farmacéutico con un objetivo de ahorro superior a los 2.500 millones de euros anuales (RD-L 4/2010; RD-L 8/2010).

De los indicadores de la Prestación Farmacéutica del SNS (medicamentos, productos sanitarios y fórmulas) con receta destacamos que el gasto farmacéutico en el 2010 ha sido de 12.211 millones $€$, lo que refleja un decrecimiento del 2,36\%, en relación con el año anterior. Con estas cifras, se ha logrado contener la subida de la factura pública en medicamentos, confirmándose la tendencia a la moderación que comenzó en abril de 2004, cuando el incremento interanual se situaba en el $11,02 \%$. Además, esta moderación en el crecimiento se ha manifestado tanto en el 
gasto medio por receta, que ha decrecido un $4,79 \%$ con respecto a 2009 , como en el número de recetas facturadas, 957 millones de recetas (unas 20 por habitante), que se han incrementado un 2,56\%, por debajo del 4,94\% del año 2010 (MSPSI, 2010).

En la Encuesta Nacional de Salud de 2006 el 69\% de los mayores de 16 años manifestaba haber tomado algún medicamento en las dos últimas semanas, cifra que llegaba al $92 \%$ en los mayores de 65 años; datos superiores a los anotados en la misma Encuesta en el año 2001 (52\% y 83\% respectivamente), lo que junto a los datos de mayor frecuentación de los servicios sanitarios muestran una sociedad más medicalizada y muy "medicamentada".

En el 2006, los fármacos más consumidos expresados en términos de D.D.D. correspondieron al grupo de los antihipertensivos, hipolipemiantes y antiulcerosos, mostrando en todos los casos una tendencia alcista (MSPSI, 2006).

Respecto a la factura hospitalaria, ralentizó su crecimiento en 2010 en relación a años anteriores (IMS, 2010). Por primera vez en los últimos años el crecimiento registrado en valores fue inferior al $10 \%$, situándose en un $8,1 \%$. Así, las ventas a PVL ascendieron a 5.779.675.493 $€$ (casi la tercera parte del coste promedio de las recetas dispensadas en las oficinas de farmacia). No obstante, esta cifra está sobreestimada, ya que no tiene en cuenta ninguno de los descuentos (comerciales o normativos) aplicados por la industria a los hospitales (RD-L 4/2010; RD-L 8/2010). Sólo cinco moléculas (tenofovir, emtricitabina, adalimumab, efavirenz y bevacizumab) han representado el 20,19\% (IMS, 2010). Finalmente, el denominado gasto farmacéutico debería contemplar no sólo el importe de multiplicar unidades consumidas por su precio, sino también el coste del fracaso terapéutico y el coste de las reacciones adversas.

Martín-Conde y colaboradores proponen una serie de avances, para mitigar esta realidad que pone en peligro la propia sostenibilidad del sistema sanitario, y son (Martín-Conde et al., 2011): 
- Afianzar la evaluación económica de la oferta farmacéutica.

A nivel de la macrogestión, cabe esperar el desarrollo normativo de la Ley incorporando la Farmacoeconomía (Oliva et al., 2008; Oliva et al., 2008a; Pinto y Abellán, 2008; Pinto-Prades et al., 2009) como criterio de peso en la negociación del precio y la decisión de la financiación pública de medicamentos. Aunque la Ley 29/2006 señala como competente a la AGEMED para realizar los análisis farmacoeconómicos y estudios de utilidad terapéutica comparada, algunos autores (Del Llano et al., 2008) abogan por la creación de un nuevo organismo en el que integrar el Comité de Utilidad Terapéutica. Además habría que optimizar la producción de las diversas agencias regionales de evaluación, sociedades científicas, etc. Por tanto, se hace imprescindible la cooperación política con un papel más activo del Consejo Interterritorial del SNS.

En el ámbito de la gestión clínica es notorio que existe una mayor consideración del impacto económico en la toma de decisiones, traducida en la programación docente, fijación de incentivos a prescriptores, etc. De especial relevancia a nuestro juicio, es la existencia del Grupo GENESIS dentro de la SEFH (GENESIS, 2011). Con la metodología desarrollada, trabajo en red y su espíritu colaborador ha logrado vertebrar el funcionamiento de la mayoría de CFT en nuestro país (Puigventós et al., 2010). Mencionar que los informes de evaluación de un nuevo fármaco con su posicionamiento terapéutico (condiciones de uso, algoritmos y recomendaciones para el intercambio terapéutico) es uno de sus objetivos. Asimismo, GENESIS ha aportado a las CFT las claves y el procedimiento para que asuman su rol institucional en la evaluación de los fármacos en situaciones especiales, de la promoción de protocolos asistenciales y la evaluación de resultados (Delgado et al., 2009a).

- Explorar nuevos sistemas de financiación en casos seleccionados.

Dada la incertidumbre sobre la efectividad, el impacto presupuestario y los datos del estudio de la evaluación económica de medicamentos, se han planteado contratos de riesgo compartido entre la Administración y el fabricante (Espin et al., 
2010). De esta manera se vinculan los ingresos del laboratorio farmacéutico a la consecución de unos objetivos acordados en volumen (acuerdos precio-volumen), utilización o resultados alcanzados con la posible innovación tecnológica. Teóricamente, estos contratos permitirían alinear los objetivos de la Administración y el fabricante para una financiación coste-efectiva, reducir la incertidumbre sobre la efectividad del nuevo fármaco con nueva información sobre sus resultados en salud, limitar el impacto presupuestario por uso en indicaciones no determinadas previamente y favorecer el acceso de posibles beneficiarios al nuevo tratamiento (Puig-Junoy y Meneu, 2005).

- Disminuir la brecha entre eficacia y efectividad es el objetivo de cualquier política sanitaria.

Constatado que la variabilidad explica más del $40 \%$ de las diferencias de uso y consumo de recursos entre entornos asistenciales; y que la adopción de un nuevo fármaco no implicará por lo general una mejor asistencia y mejores resultados si no se modifican otros factores, se hace necesario maximizar todas esas etapas del proceso clínico (Ortún, 2003). Desde el prisma farmacoterapéutico, es preceptivo: a) Garantizar la seguridad clínica, especialmente en el ámbito hospitalario incorporando las nuevas tecnologías (prescripción electrónica, lectores de códigos de barras; procedimientos explícitos para mejorar la seguridad de la utilización de medicamentos de alto riesgo, etc.). b) Conciliar las discrepancias entre la medicación que recibe el paciente y lo que realmente tiene prescrito, consecuencia de su tránsito por los diferentes niveles asistenciales (atención primaria, ingresos hospitalarios, traslados de unidad, alta hospitalaria, urgencias, etc.). La conciliación repercute directamente en la efectividad y los costes de los tratamientos. c) Implantar programas de SFT al objeto de disminuir los RNM (Calleja MA et al., 2008; Delgado, 2007; Delgado et al., 2009; Silva-Castro et al., 2010).

\section{- Escuchar la voz de los pacientes.}

Por último y no menos importante, deben buscarse los cauces y para incorporar las preferencias de los pacientes en la toma de decisiones clínicas (Pinto 
et al., 2004). Como manifiesta A. Segura: "sería deseable la participación activa de los ciudadanos en los ámbitos de la salud y la sanidad al objeto de materializar las preferencias derivadas de la percepción de las necesidades en salud y de optar entre distintas alternativas que afectan a la eficiencia, el coste oportunidad y la justicia a la hora de distribuir los recursos comunes" (Segura, 2010).

La sostenibilidad del Sistema Nacional de Salud (SNS) a medio y largo plazo, y las condiciones de esta sostenibilidad en términos de extensión y calidad de los servicios que presta, depende en buena medida de cómo pueda afrontar las presiones derivadas de la actual situación de crisis económica. En todo caso, la necesidad de priorizar actuaciones, minimizar el despilfarro y mejorar la eficiencia interna del SNS es hoy más visible y más perentoria que nunca. Con estos objetivos declarados, el gobierno de España, con el respaldo de las comunidades autónomas en el Consejo Interterritorial, ha puesto en marcha diversas actuaciones a través de Reales Decretos Ley (RD-L 4/2010; RD-L 8/2010), incluyendo, entre otras medidas, la reducción de los salarios del personal del SNS, la reforma del sistema de precios de referencia, la reducción del precio industrial de los genéricos, descuentos obligatorios en los medicamentos sin precio de referencia, y descuentos y modificaciones en los márgenes de las oficinas de farmacia (Peiró et al., 2011).

Peiró y colaboradores proponen una serie de medidas para facilitar la sostenibilidad de nuestro sistema sanitario, entre las que podemos enumerar 9 de las 112 propuestas y evaluadas por el panel de expertos que participaron en el estudio, las cuales no se incluyeron en los Reales Decretos mencionados, y fueron: 1) Convencernos de que no se puede mejorar la gestión sanitaria pública sin controlar la corrupción y la partitocracia; 2) Contundente reorganización del terciarismo basada en evidencias sobre la relación entre volumen, resultados y costes; 3) Replantear las actividades preventivas que no aportan valor añadido (chequeos, revisiones de empresa y otras), especialmente en la propia Administración; 4) Gestión de la demanda, priorización según necesidades: urgencias, exploraciones, visitas, intervenciones; priorización listas de espera, cartera de servicios; 5) Implicar a los pacientes (sobre todo con enfermedades 
crónicas y hábitos no saludables) en los autocuidados; 6) Definir la cartera de servicios «basada en la evidencia»; 7) Reforzar el papel de la atención primaria como filtro de la especializada; 8) No abrir más hospitales (sobre todo pequeños); y 9) Concentración de la oferta: a) módulos de guardia; b) tecnologías punta; c) concentración terciarismo; d) reducir facultades de medicina y escuelas de enfermería; e) reducir/concentrar los centros de investigación (Peiró et al., 2011).

\subsection{Gestión clínica y atención farmacéutica.}

Por lo expuesto anteriormente, surge la necesidad de adaptar nuestros modelos de salud, no sólo en cambios que se traduzcan en mejoras de la práctica médica, sino también en la creación de nuevas fórmulas de gestión para las organizaciones sanitarias. De esta forma, resulta preciso desarrollar y aplicar iniciativas que permitan afrontar el reto que se nos plantea. Estas medidas pueden abordarse a nivel macro, es decir, a través de diferentes políticas de salud, si bien, también pueden ser aplicadas desde una dimensión meso o desde la perspectiva de las propias organizaciones sanitarias, pero lo que realmente resulta crucial es avanzar hacia organizaciones horizontales, dando valor a los microsistemas y con ello a los profesionales sanitarios que lo conforman. El centrar la toma de decisiones en el propio clínico, en aras del reconocimiento de sus valores profesionales, no es más que una apuesta hacia la consecución de organizaciones más eficientes, lo que implica no sólo la prestación de una asistencia de calidad, sino hacerlo, además, sin malgastar los recursos (Amat-Díaz et al., 2011; Bernal Delgado y Ortún-Rubio, 2010).

La idea de gestión clínica puede formularse en un doble sentido: hacer más clínica la gestión y hacer más gestora la clínica; a través de ambos componentes se facilita la identificación de aspectos importantes de este patrón. En efecto, para hacer más clínica la gestión, el reto se centra en tres ámbitos (Cosialls-Pueyo, 2000; Repullo-Labrador, 2006): 
- gestionar lo esencial, es decir la función de producción, reforzando el papel y dinamismo de las Direcciones Asistenciales, y los objetivos e información de efectividad y calidad;

- la gestión del conocimiento, para aportar desde la red sanitaria una política científica y unos instrumentos de apoyo a las mejores prácticas y a la excelencia; $y$,

- la gestión de las interdependencias de red, para abordar los problemas de cronicidad y de respuestas integrales a patologías, desde una infraestructura y una logística de red.

Introducir la dimensión gestora en el mundo clínico supone avanzar en varios sentidos (Repullo Labrador, 2006):

- buscar formas de organizar los centros, servicios y unidades, para que domine la cooperación, la horizontalidad y el trabajo por procesos;

- fomentar un liderazgo desde el mundo clínico, que profesionalice los retos y defienda los proyectos de cambio organizativo en su entorno profesional; $y$,

- mejorar y racionalizar las decisiones clínicas, buscando mejorar las dimensiones de efectividad, eficiencia, calidad y satisfacción de los pacientes.

Aceptar las bases de la gestión clínica conlleva, inherentemente, para los profesionales sanitarios, la asunción de responsabilidades más allá de lo estricto de su tarea y con ello la obligación de disponer de una aptitud clínica superior, que aúne sus habilidades cotidianas junto a conocimientos específicos, en albor de un mayor compromiso institucional y social (Cosialls-Pueyo, 2000). La medicina y la gestión, de esta forma, deben caminar juntas con el único fin de conseguir los mejores resultados para los pacientes y al mismo tiempo conseguirlos de la mejor forma posible. Es el camino, por tanto, para garantizar la excelencia, meta de 
cualquier profesional sanitario.

La gestión clínica, como paradigma emergente de esta década, es la piedra angular de las estrategias de mejora del sistema, y de búsqueda de sostenibilidad y excelencia. Su avance depende de la factibilidad de una nueva alianza entre políticos, gestores, médicos y resto de profesionales sanitarios, alineando intereses, compartiendo visiones y minimizando los conflictos de interés.

Hemos de pasar a ser, por tanto, los protagonistas del cambio, los elementos clave para asegurar que nuestro sistema sanitario persevere. No podemos olvidar que cada una de las decisiones que tomamos en nuestra práctica diaria va vinculada a una parte del gasto sanitario, y en un entorno de incertidumbres, debemos ser el aval para la racionalización de los recursos, aunque siempre sin eludir el beneficio de aquellos a los que se dirige nuestra atención y nuestra razón de ser, los pacientes.

Con la incorporación de la atención farmacéutica como paradigma del rol del farmacéutico, le ha supuesto un compromiso, corresponsabilizándose con el resto del equipo multidisciplinar en el proceso farmacoterapéutico, en la mejora de la calidad de vida de los pacientes (Helper y Strand, 1990). Responsabilidad que siempre ha estado en las actuaciones del farmacéutico de hospital y en las decisiones que toma diariamente, independientemente de su lugar de actuación (Carrera-Hueso y Giráldez, 1995).

La atención farmacéutica no es una tecnología, desde el punto de vista economicista, fácil de medir. En efecto, existen multitud de estudios que demuestran su utilidad y eficiencia para el sistema sanitario, pero muchos están salpicados de sesgos de diseño, análisis incorrectos, muestras pequeñas y periodos de seguimiento excesivamente cortos. Por lo que no debemos caer en un excesivo entusiasmo y continuar perseverando con la labor de demostrar su valor añadido, esto es su eficiencia (Peiró, 2006).

El farmacéutico de hospital debe ser capaz de obtener e interpretar las 
variables o resultados en salud, que incluyen los de tipo clínico, epidemiológicos o económicos, pero que también debe considerar los de tipo humanístico, que son los de calidad de vida relacionada con la salud y de satisfacción percibida por los pacientes (Carrera-Hueso y Giráldez, 1995).

Una organización que aprende es experta en crear, adquirir y transmitir conocimiento, y capaz de adaptarse a ideas y conocimientos en cada momento. La gestión del conocimiento es una herramienta clave para que las organizaciones sean excelentes, y esencial en las sanitarias. Los SF son elementos vertebradores del hospital y tienen una visión global del centro hospitalario. Además, son generadores y transmisores de conocimiento, siendo esté gestionado. Cada SF debe conocer y desarrollar su capital intelectual, transformador, innovador y orientado siempre hacia el paciente (López-González y Sánchez-Sánchez, 2007). Con ello añadirá valor y calidad a la mayoría de procesos asistenciales, tanto los desarrollados dentro de los mismos como los compartidos.

La organización horizontal significa que se deben alinear las necesidades de los profesionales y los pacientes con las estrategias de la institución, y no solamente implantar una gestión por procesos. Lo que implica impulsar estrategias de innovación y de flexibilidad operativa y financiera en su organización. Para innovar, es necesario pensar en los resultados que se obtienen tanto en los propios SF como los obtenidos en otros servicios y departamentos del hospital, consiguiendo una mejor gestión asistencial y se podrá hacer partícipe a los demás profesionales sanitarios de su consecución y siempre que sea posible también a los pacientes. Con ello fomentamos la creación de la cadena de la innovación en la que participen tanto empleados como clientes, internos y externos, en nuestro SF; que facilita los mecanismos de adaptación al cambio cultural necesario y la adopción de estrategias operacionales claras y eficientes para lograr organizaciones excelentes.

Orientarse a la innovación implica un compromiso a largo plazo con la creencia de que lo único constante es el cambio y que la flexibilidad es la cualidad que permite adaptarse a esos cambios. 
Orientarse hacia el paciente no significa solamente tenerle presente en nuestros pensamientos sino hacerle realmente participe conociendo de primera mano sus opiniones e inquietudes. Para entender el concepto de satisfacción del paciente se ha optado por un enfoque pragmático con las siguientes siete dimensiones: empatía, información que reciben, competencia profesional, capacidad de respuesta, accesibilidad, confort y estado de las instalaciones y resultado del tratamiento. Para su medición utilizamos cuestionarios que permiten valorar el punto de vista del paciente en referencia a las dimensiones citadas, obteniendo resultados relacionados con su estado físico, funcional y psíquico, y también pueden ser de tipo económico y social. Por eso es importante seleccionar adecuadamente la muestra diana a la que dirigimos el estudio y su tamaño muestral para conseguir resultados válidos que orienten la toma de decisiones para realizar acciones correctoras o preventivas que mejoren nuestras actividades (Lorenzo, 2006).

Los SF son un componente más de la continuidad asistencial y, por tanto, no se puede centrar en ellos todo sobre la cultura de seguridad y la resolución de los fallos de los sistemas para conseguir organizaciones sanitarias excelentes y seguras. Sin embargo, sí deben asumir el reto mediante el liderazgo y su compromiso para hacer del hospital un sitio cada vez más seguro para los pacientes, y concretamente en la utilización de los medicamentos. En efecto, el liderazgo farmacéutico es el elemento clave para el éxito del programa de seguridad de la medicación, desde un enfoque multidisciplinar, implantando prácticas y desarrollando estrategias para conseguir el objetivo de reducir los problemas relacionados con los medicamentos y qué estos no lleguen al paciente. Para crear una infraestructura con sistemas visibles y sostenibles para la gestión segura de los medicamentos, es necesario que las principales actuaciones sean aplicadas por los líderes farmacéuticos (Amat et al., 2011; Otero López, 2010). 


\subsection{Estudios de resultados sanitarios.}

La gestión sanitaria se puede definir como una variedad de métodos de financiar y organizar la distribución de cuidados sanitarios con el objetivo de contener el gasto controlando la provisión de los servicios (Inglehart, 1994).

Relman sugirió que se entró a partir de los 90 en la tercera edad del cuidado médico: la era de la valoración y la justificación. Esta era siguió a la era de contención del gasto, entre los 70 y 80 , precedida por la era de la expansión, entre los 40 y 60 del siglo anterior. Este cambio fue debido a que con las políticas de contención del gasto no se ha podido controlar el incremento del gasto sanitario (Relman, 1988). Actualmente nos encontramos, tras haber traspasado la era del conocimiento y de la evidencia para la toma de decisiones clínicas, en un momento de reformas sanitarias buscando el mejor modelo sanitario sobre el que pivota la decisión de agencia de los médicos sin entrever un futuro claro y esperanzador (Relman, 2009; Peiró et al., 2011).

Tradicionalmente la medicina estaba más preocupada por sanar o paliar el efecto de las enfermedades que de mejorar el bienestar social. Para ello utilizaba tres indicadores para evaluar sus resultados: mortalidad, morbilidad y coste. Estos indicadores también servían para la toma de decisiones farmacoterapéuticas y su posterior seguimiento (Carrera-Hueso, 2000).

Si tenemos en cuenta la definición de salud dada por la OMS en 1948, "estado completo de bienestar físico, mental y social, no solamente la ausencia de la enfermedad" (WHO, 1948), éstos indicadores no son útiles ni para monitorizar el resultado de nuestras actuaciones ni para disminuir el coste total de los tratamientos farmacológicos. Por ello se diseñaron modelos en los que se propusieron medir sistemáticamente en la práctica clínica diaria el grado de funcionamiento y de bienestar de los pacientes, orientándose por tanto a un modelo de gestión de resultados sanitarios (Ellwood, 1988). 
La calidad de los servicios sanitarios depende de la técnica, considerada como el conjunto de habilidades y conocimientos, y de las relaciones interpersonales, contemplándose la opinión de los pacientes acerca de sus tratamientos (Donabedian, 1988). Hay una conexión entre estructuras, procesos y resultados sanitarios, y solamente podemos aumentar la calidad de los servicios sanitarios prestados si mejoramos los procesos a través de programas de garantía de calidad o mejorando los resultados sanitarios a través de los estudios de resultados (outcomes studies), considerados como los efectos de los cuidados sanitarios sobre los pacientes y poblaciones. (Donabedian, 1988).

Kozma y colaboradores diseñaron un modelo gráfico para comprender el complicado proceso de la toma de decisiones farmacoterapéuticas en las que no solo se miden los resultados clínicos en el tratamiento de los pacientes sino también los resultados económicos y humanísticos del mismo (Kozma et al., 1993). El modelo contempla los resultados clínicos como los derivados de una enfermedad o tratamiento (morbilidad y mortalidad), los económicos son aquellos que comparan los costes y las consecuencias de las distintas alternativas, y los humanísticos son las consecuencias de la enfermedad sobre el estatus funcional del paciente o medidas sobre la calidad de vida en varias dimensiones. También se contempló en el modelo la existencia de intermediarios o modificadores del tratamiento que pueden afectar a los resultados del mismo, y su importancia puede ser en determinadas ocasiones muy alta, como por ejemplo el incumplimiento terapéutico o el mal uso de los medicamentos puede suponer un alto coste por aumento de los ingresos hospitalarios (Johnson y Bootman, 1995; Delgado et al., 2009).

\subsubsection{Eficacia, efectividad y eficiencia}

La eficacia hace referencia al impacto o efecto de una acción llevada a cabo en condiciones óptimas o experimentales. Responde a la pregunta sobre cuál es la capacidad de un curso o acción sanitaria (bajo condiciones de uso y de aplicación ideales) para mejorar el nivel de salud del individuo o colectivo. La eficacia no ofrece dudas, tiene validez universal y debe ser invariante respecto del lugar y del tiempo. 
Normalmente se extraen los datos de eficacia desde los ensayos clínicos controlados y aleatorios realizados (Drummond et al., 2005; Sacristán et al., 1995; Gimeno et al., 2006). Por ejemplo, la eficacia de un fármaco antihipertensivo en reducir la TA sistólica y diastólica fue del 95\% en un ensayo clínico en el que se incluyeron pacientes adultos hipertensos y sin otro tipo de co-morbilidad.

El concepto de efectividad hace referencia al impacto que se alcanza a causa de una acción llevada a cabo en condiciones habituales. Se refiere así a la posibilidad de que un individuo o colectivo se beneficien de la aplicación de un procedimiento farmacológico o de cualquier práctica médica (Bootman et al., 2004; Drummond et al., 2005). Siguiendo con el ejemplo anterior, supongamos que en la población atendida por nosotros los pacientes tienen un grado de cumplimiento del $60 \%$, posiblemente debido a los efectos adversos al antihipertensivo, y además la mitad de la población a la que tratamos son mayores y su grado de eficacia en este tipo de pacientes es del 70\%: La efectividad sería igual a $0,95 \times 0,6 \times 0,5+0,70 \times$ $0,6 \times 0,5=0,495$; es decir, la efectividad del fármaco es del $49,5 \%$ para nuestra población de pacientes. El nivel de efectividad depende del espacio y del tiempo, no teniendo carácter universal (Gimeno et al., 2006).

Por último, la eficiencia viene referida a la producción de bienes y servicios que la sociedad valora más al menor coste social referido. Se emplea de manera intuitiva como equivalencia de la relación óptima entre el resultado esperado y la inversión que ésta requiere (Gimeno et al., 2006). Su valor puede determinarse mediante la relación del cociente entre los resultados obtenidos y el valor de los recursos empleados medidos en unidades monetarias. Por lo que la eficiencia estará condicionada a como medimos los resultados de nuestras intervenciones que principalmente son cuatro, en unidades naturales como la mortalidad, paciente controlado, etc.; en unidades monetarias, en calidad de vida; dando paso a los diferentes tipos de evaluaciones económicas (Bootman et al., 2004; Drummond et al., 2005). Siguiendo con el ejemplo anterior, supongamos que el tratamiento de 100 pacientes cuesta $1500 €$ con el antihipertensivo, entonces la eficiencia del fármaco 
en nuestra población sería de 1500/0,495 $=3.030,3 €$ por paciente, es decir nos cuesta controlar la hipertensión de un paciente más de tres mil euros.

Los estudios de eficacia y efectividad son previos a la evaluación de la eficiencia, de modo que no se puede realizar una evaluación económica de algo cuya eficacia no esté demostrada. También es previa la demostración de la seguridad relativa al medicamento evaluado. Es necesario evaluar la evidencia científica de la eficacia o efectividad de las alternativas que se van a comparar, en caso contrario la evaluación económica sería ineficiente (Gimeno et al., 2006; Bootman et al., 2004; Drummond et al., 2005).

La eficiencia es un concepto relativo y por tanto debe de ser comparado con otra alternativa, sea otro fármaco o tecnología o simplemente la opción de no hacer nada. La ineficiencia equivale a despilfarro innecesario de recursos, a inadecuación por sobreutilización o a aplicaciones de los mismos en actividades que generan superfluos gastos, excesivos o desmedidos costes, o tienen escasos rendimientos (Bootman et al., 2004; Drummond et al., 2005; Gimeno et al., 2006).

\subsection{Estudios farmacoeconómicos}

El crecimiento exponencial del gasto sanitario y en medicamentos, ha sido principalmente financiado con cargo a fondos públicos en nuestro país. Persiste gran incertidumbre sobre los efectos relevantes para la salud de muchas de las tecnologías y procedimientos utilizados en la actualidad y se está produciendo una creciente asignación de recursos a la asistencia sanitaria, atribuible al uso de tecnologías insuficientemente evaluadas. En efecto, en una situación económica de contención de costes y de crisis económica generalizada, es un imperativo ético más acuciante si cabe, la necesidad de utilizar los recursos de manera lo más eficiente posible, siendo inexcusable la evaluación de las nuevas tecnologías a incorporar (Peiró et al., 2011).

El método general de evaluación económica ha experimentado con el propio progreso científico y con la penetración de las ciencias económicas en el campo de 
la salud, modificaciones de adaptación a nuestro campo, que ha trascendido al concepto ya clásico como instrumento originario del análisis coste-beneficio, creando un cuerpo de doctrina propio y específico a sus peculiaridades (CarreraHueso y Ronchera-Oms, 1996).

Las evaluaciones económicas sanitarias presentan dos características básicas al margen de las actividades a las que se aplique, y son: la primera tiene que ver con los factores productivos (inputs) como de los productos (outputs) siendo la relación entre costes y beneficios la que nos permita llegar a una decisión; la segunda, el análisis económico está directamente relacionado con la elección, basándose en criterios más implícitos al disponer de mayor información. En resumen la evaluación económica conlleva un análisis comparativo de las acciones alternativas tanto en términos de costes como de beneficios (Drummond et al., 2005; Gimeno et al., 2006).

Los estudios farmacoeconómicos identifican, miden y comparan los costes (recursos consumidos) y las consecuencias (clínicas, económicas y humanísticas) de los productos farmacéuticos y servicios prestados (Bootman et al., 2004).

\subsubsection{Diseño, alternativas, perspectiva y horizonte temporal}

La definición del estudio es la primera fase para realizar un estudio farmacoeconómico y constituye la base del protocolo de investigación, conteniendo la pregunta a resolver de forma clara, precisa y objetiva. Para hacer la pregunta correctamente se deben tener presente la perspectiva, el horizonte temporal y los tipos de costes que se tendrán en cuenta y los resultados que se medirán y en qué tipo de población o muestra (Soto, 2002; Drummond et al., 2005).

El diseño es la forma en la que se estructura el estudio, el cómo, el cuándo y el dónde se obtienen los datos necesarios para una investigación y la manera de llevarla a cabo, teniendo en cuenta la clase de datos que son tenidos en cuenta, los métodos para obtenerlos y la forma en qué son analizados e interpretados. Pueden diferenciarse 3 tipos de diseños (Soto, 2002): estudios prospectivos, estudios 
Introducción

retrospectivos y estudios predictivos (modelizaciones). También pueden ser longitudinales y transversales dependiendo del momento de medición; experimentales u observacionales si existe intervención del investigador; y dependiendo si se realizan en el mundo ideal o real: en ensayos clínicos controlados, y ensayos pragmáticos o naturalísticos (Drummond et al., 2005; Gimeno et al., 2006).

La tecnología objeto de evaluación ha de ser claramente definida y descrita en todos sus aspectos, siendo imprescindible precisar el lugar de aplicación, el personal encargado de desarrollarla, la planificación temporal y, lo que es muy importante, la población objetivo a la que se dirige. La población objetivo se definirá sobre la base de criterios como el sexo, la edad, el ámbito geográfico o la condición médica de los sujetos (Soto, 2002).

Cuando exista evidencia de diferencias en los efectos o los costes de la tecnología para diferentes subgrupos de la población objetivo, estos subgrupos han de ser identificados y, si se estima oportuno, han de constituir los sujetos de análisis o evaluaciones separadas, que se añadirán a la efectuada para el conjunto de la población objetivo (Soto, 2002).

Las alternativas en las que se compara la tecnología que deseamos estudiar pueden ser: el "no hacer nada", las técnicamente posibles, y otras, pero deben siempre estar justificadas por los investigadores. Lo más recomendable es utilizar como comparativa las opciones más utilizadas o tradicionales (Soto, 2002; Gimeno et al., 2006). Por ejemplo, para comparar una nueva estatina, en prevención primaria o secundaria de la enfermedad cardiovascular, no sería muy lógico ni ético compararla con no hacer nada ya que existen evidencias de la efectividad de otros fármacos ya comercializados del grupo, por lo que sería recomendable compararla con atorvastatina, medicamento más utilizado en nuestro país y en el resto del mundo.

La tecnología objeto de evaluación ha de ser comparada, al menos, con la intervención existente -la práctica habitual o status quo- y con la opción "no hacer 
nada" -o, en su lugar, la intervención mínima-. La práctica habitual puede ser una única intervención -la dominante, si es posible identificarla- o bien una combinación de todas las existentes. Estos términos de comparación para el análisis incremental pueden consistir en intervenciones de un tipo de tecnología diferente a la evaluada (Puig-Junoy y Pinto, 2008).

La perspectiva se refiere al punto de vista desde el que se llevan a cabo los estudios farmacoeconómicos identificando al agente social que los promueve y se supone utilizará los resultados para la toma de decisiones. Los principalmente utilizados son: la sociedad en su conjunto o perspectiva social, unidades de gestión clínica proveedoras de servicios públicos o privados (principalmente el hospital), las aseguradoras, los provisores de recursos financieros públicos o privados, los pacientes o sus asociaciones, los empresarios, las autoridades sanitarias o las organizaciones socio profesionales (Drummond et al., 2005).

Una misma intervención sanitaria examinada desde distintas perspectivas puede dar lugar a diferentes criterios de decisión y modificar la elección. Por ejemplo, al realizar un estudio de una enfermedad crónica muy invalidante (psoriasis o artritis reumatoide) debe incorporarse la pérdida de días de trabajo desde la perspectiva social o del paciente o familiar, pero no se incluiría desde la perspectiva del hospital. Los especialistas recomiendan utilizar la perspectiva más amplia posible, la social, para que los resultados de los estudios sean más generalizables y aplicables en casi todos los supuestos y además es ajena al interés particular de los agentes intervinientes e implicados (Drummond et al., 2005; Gimeno et al., 2006; Bootman et al., 2004; Sacristán et al., 1995).

El ámbito de análisis de la evaluación económica ha de acotarse, tanto en lo que se refiere al tipo de efectos que se incluirán en el estudio -no sanitarios vs. sanitarios y, dentro de éstos, beneficios físicos, psíquicos, emocionales-, como en lo que respecta a los individuos, además del paciente o destinatario directo del programa, cuyos costes y beneficios serán objeto de medición y valoración. 
El horizonte temporal es el periodo temporal en el que se van a valorar las consecuencias o resultados en salud y los recursos de cada una de las alternativas contempladas (Gimeno et al., 2006). Por ejemplo, hay enfermedades crónicas que el horizonte temporal será de 5 años o más, sin embargo en Unidades de Cuidados Críticos sería impensable contemplar más allá de 2 ó 3 meses.

El horizonte temporal del análisis -el mismo para los costes y para los beneficios- ha de ser tal que garantice la inclusión de los principales efectos económicos y sanitarios del programa. Si es preciso, se recurrirá a la obtención de datos modelizados que permitan la extensión del horizonte analítico.

La tasa de descuento refleja la preferencia temporal y el rendimiento de las inversiones en una sociedad, por lo que es utilizada para la actualización del dinero o de los costes, y algunas veces de los beneficios aunque este extremo está todavía en discusión si incluirlos o no. Cuando el periodo estudiado es superior a un año, los costes contemplados en cada opción deben ser actualizados. La actualización tiene su fundamento en la ley de de subestimación de las necesidades futuras, o preferencia de retrasar los costes y anticipar los beneficios, lo que significa que el valor de un bien es tanto menor o es menos apreciado por los individuos, cuanto más lejana se encuentra su disponibilidad en el tiempo (Drummond et al., 2005; Gimeno et al., 2006).

\subsubsection{Costes}

El concepto de coste debe emplearse cuando se pretende hacer una valoración de los recursos aplicados a la producción de un bien, a la prestación de un servicio o la satisfacción de una necesidad. Se calculan multiplicando las cantidades de los factores utilizados por su respectivo coste unitario, referidos al año de referencia elegido. El coste oportunidad es aquel que al emplear los recursos en una determinada opción se renuncia a unos beneficios que se obtendrían al utilizarlos en otras. El ejemplo más típico es el dinero o el interés del capital que nos dejaría de dar el banco por destinar la inversión en otro tipo de inversión (Raferty, 2000). 
Introducción

Según el ámbito considerado podríamos hablar de costes individuales, institucionales y sociales. Todos los costes pueden ser fijos o variables. Los costes se clasifican en dos grandes grupos: tangibles e intangibles (Bootman et al., 2004; Drummond et al., 2005; Gimeno et al., 2006).

Costes intangibles: son aquellos que no pueden ser valorados a través de los mecanismos del mercado, y que soportan los pacientes (sufrimiento, dolor, miedo, malestar, incomodidad, insatisfacción o pérdida de ocio) o el conjunto de la sociedad (alarma, inquietud, preocupación social con la institución). Los costes intangibles son muy importantes pero en la mayoría de los casos no se incorporan a los cálculos por la dificultad de obtenerlos y porque su valoración es siempre polémica.

Costes tangibles: son aquellos que son valorados de forma explícita a través de los precios de mercado, como son los de personal, amortizaciones, consumos, suministros, etc. Los costes tangibles pueden ser: directos e indirectos.

Costes tangibles directos: constituyen transacciones que afecta a recursos del sistema sanitario o soportan agentes sociales integrados en el mismo. Deben distinguirse entre sanitarios o médicos y no sanitarios.

Costes tangibles directos sanitarios ó médicos: incluyen aquellos que llevan a cabo la actividad sanitaria y directamente relacionados con la obtención de resultados o que son empleados como productos intermedios propios del sector. Aquí se incluyen normalmente los costes que soportan las instituciones sanitarias como el salario del personal médico y de enfermería, los consumos de medicamentos y productos sanitarios, amortizaciones de instalaciones y equipos, cocina, limpieza, entre otros.

Costes tangibles directos no sanitarios o médicos: son aquellos que inciden en el bolsillo del paciente, amigos o familiares como son los desplazamientos, adaptaciones del hogar, coste de cuidadores, entre otros.

Costes tangibles indirectos: Son aquellos que afectan 0 repercuten negativamente en otro tipo de sectores económicos, y no en el sanitario; como es la 
Introducción

pérdida de productividad debida al absentismo laboral provocado por la convalecencia, y a la propia pérdida de capacidad para el trabajo, temporal o definitiva (muerte).

Los costes medios son el resultado de dividir los costes totales entre el número de pacientes, mientras que los costes marginales miden el aumento en los costes totales asociado a un incremento unitario -marginal- en el resultado (output), es decir, en la cantidad de población a la que alcanza el tratamiento. La magnitud relevante a la hora de valorar la extensión de un programa sanitario es el coste marginal, no el coste medio por paciente (Raferty, 2000).

La clase de costes que deben incluirse en una evaluación económica depende de factores como la perspectiva adoptada, el horizonte temporal, entre otros. Se deben incluir todos los costes que sean relevantes y puedan diferir entre las distintas alternativas estudiadas. Actualmente se utilizan costes obtenidas de bases de datos por procesos, públicas o privadas, como son los costes por GRD ajustados por complejidad (Bootman et al., 2004; Drummond et al., 2005).

El grado de precisión con que medir los recursos puede variar, desde la estimación detallada de cada componente y asignación de un precio para cada uno de ellos con objeto de obtener el coste (micro-costes), hasta el enfoque de costes brutos cuando no es necesaria tanta precisión. Por ejemplo se engloba aquí la agrupación de recursos utilizados en el tratamiento de un paciente hospitalizado a través del coste de la estancia o a través de GRD, una técnica de clasificación de los pacientes en función del diagnóstico. También será importante definir si el uso de recursos se contabiliza o no específicamente para cada paciente., considerando que el consumo de cada uno es distinto. Se puede asumir que es constante para todos los pacientes que reciben el tratamiento si utilizamos datos agregados (Raferty, 2000; Drummond et al., 2005).

No deben computarse los costes sanitarios futuros que no estén relacionados con el programa evaluado, como tampoco se han de incluir los costes que sean comunes a todas las alternativas, ni los costes estrictamente vinculados a la 
realización de los ensayos controlados. Una evaluación económica que asuma la perspectiva social en el análisis no debe en ningún caso computar como coste los pagos por transferencias, pues no implican un coste de oportunidad.

Cada elemento de coste debe ser expresado en unidades naturales. Los datos sobre uso de recursos pueden proceder de fuentes primarias -si los costes se miden al tiempo que se recogen los datos de efectividad-, o bien obtenerse retrospectivamente -a partir de una revisión de la literatura-. Cuando se tiene la sospecha fundada de que la inclusión o no de un elemento del coste no influirá significativamente en los resultados puede estar justificado renunciar al esfuerzo suplementario que exigiría su medición (Carrera-Hueso y Ronchera-Oms, 1996; Raferty, 2000).

Los recursos empleados han de valorarse según su coste de oportunidad. En principio, los precios de mercado constituyen una buena aproximación a dicha magnitud. Cuando los precios de mercado no se consideren fiel reflejo de los costes de oportunidad se ha de recurrir a métodos de ajuste de dichos precios. El tiempo consumido por el paciente y sus familiares -para el que no existe un precio-, atendiendo al criterio del coste de oportunidad se debería valorar por el salario medio de un individuo de sus mismas características. En el caso de individuos que no formen parte de la población activa, no existe un método indiscutido para valorar el tiempo, siendo posible recurrir al método del coste de reemplazo (Puig-Junoy y Pinto, 2008).

\subsubsection{Beneficios}

Los beneficios relevantes a la hora de llevar a cabo un estudio de evaluación económica se pueden clasificar en tres categorías: beneficios sanitarios -es decir, mejoras en la salud-, ganancias de productividad y mejoras en la calidad de vida no directamente relacionadas con mejoras en la salud. Estos efectos se consideran beneficios tanto si son experimentados por el propio paciente al que va destinado el programa como si los disfrutan otros individuos próximos a él -familiares, por ejemplo- (Puig-Junoy y Pinto JL 2008). 
La expresión de los beneficios de una tecnología sanitaria en unidades naturales que midan los resultados intermedios -tasa de glucemia, nivel de presión arterial- es una opción atractiva por su aparente lógica, pero presenta numerosos problemas: no se basa en las preferencias, no permite obtener conclusiones sobre asignación de recursos y no es útil en los casos en que la efectividad del tratamiento es multidimensional (Soto, 2002; Sacristán et al., 1995).

Una alternativa a las unidades naturales de medida de la efectividad son las escalas de calidad de vida relacionada con la salud (CVRS) (Puig-Junoy y Pinto JL 2008). Estas medidas son útiles en el contexto de la evaluación económica siempre y cuando cumplan una serie de requisitos: a) que los beneficios se midan en una escala común -para que se puedan agregar-; b) que los intervalos de calidad de vida sean comparables con intervalos de cantidad de vida; c) que se puedan combinar con la duración; y d) que estén basadas en las preferencias.

Los años de vida ajustados por la calidad (AVACs) son una medida de los resultados de los programas sanitarios que combina los dos componentes de una mejora en la salud: cantidad $(Y)$ y calidad $(Q)$ de vida. Los AVACs suponen que la utilidad que proporciona un estado de salud que combina ambas dimensiones se puede expresar como el producto de ambos componentes: la utilidad -el valorasignada a un estado de salud, $V(Q)$, y el tiempo en que el sujeto vive en dicho estado, Y (Puig-Junoy y Pinto JL 2008).

Los AVACs constituyen la unidad de medida por excelencia en el análisis coste-utilidad. Existen, sin embargo, otras medidas que combinan cantidad y calidad de vida y que son, en principio, aptas para ser utilizadas en un análisis costeutilidad, si bien su uso hasta la fecha ha sido muy limitado. Se trata de los años de vida ajustados por la discapacidad (DALYs), las vidas jóvenes salvadas equivalentes (SAVEs) y los años de vida equivalentes (HYE) (Drummond et al., 2005).

Las preferencias que resultan de interés en la evaluación económica son las del conjunto de la población, ya que el estudio se lleva a cabo desde una perspectiva social. Por ello los valores de utilidad empleados en un análisis coste- 
utilidad, así como los valores de disposición a pagar en caso de un análisis costebeneficio, deben ser obtenidos de una muestra de la población en general debidamente informada o instruida. Sólo si se tiene la certeza de que de ello no se derivarán sesgos y que, por tanto, no existirán diferencias significativas respecto del recurso a la población en general, se pueden obtener las preferencias de subgrupos de la población particulares como los pacientes o el personal sanitario (Drummond et al., 2005).

La distribución de los beneficios debe ser especificada en el análisis de modo que sea posible conocer qué sujetos disfrutarán la mayor parte de los beneficios derivados de la adopción de la tecnología evaluada. En principio, la asignación de diferentes ponderaciones a los beneficios percibidos por distintos grupos de individuos es una opción que debe valorar el responsable de la toma de decisiones, no el autor del estudio de evaluación económica. No obstante, dado que la no asignación de pesos distributivos supone, de hecho, la asunción de un criterio de equidad implícito -diferente según la modalidad de evaluación elegida-, no está de más que en el estudio se dedique un espacio a considerar las cuestiones de equidad (Rodríguez-Barrios, 2004; Puig-Junoy y Pinto JL 2008).

\subsubsection{Tipos de evaluaciones sanitarias}

La evaluación de las tecnologías sanitarias se refiere a una forma amplia de investigación que examina las consecuencias clínicas, sociales, económicas y éticas que se producen a corto y largo plazo, derivadas del uso de tecnologías, tanto directas como indirectas, y tanto sobre los efectos deseados como los no deseados. Debe diferenciarse por tanto la evaluación económica de otras como la evaluación clínica (considerando al paciente de manera individualizada), la evaluación epidemiológica (para grupos poblacionales), la evaluación moral, social o política (equidad) y la evaluación financiera que mide las alternativas desde la óptica de la calidad y cantidad de los recursos o medios financieros empleados (Drummond et al., 2005; Gimeno et al., 2006). 
La farmacoeconomía fue definida por Townsend en 1897 como la descripción y análisis de los costes de los tratamientos farmacológicos a los sistemas sanitarios y la sociedad (Townsend,1987). Se trata por tanto de una evaluación económica de los medicamentos y/o de los servicios, farmacéuticos o no, asistenciales prestados, ya que los estudios farmacoeconómicos comparan los costes (recursos utilizados) y consecuencias (resultados humanísticos, clínicos y económicos) de los fármacos y servicios farmacéuticos. En esencia, los análisis farmacoeconómicos utilizan herramientas para examinar el impacto, deseable o no, de las alternativas terapéuticas disponibles y otras intervenciones sanitarias (Drummond et al., 2005).

Los tipos de evaluaciones económicas pueden ser parciales o completas, siendo éstas últimas las que comparan dos o más alternativas y se examinan tanto los costes como las consecuencias de las mismas y que se utilizan en los estudios farmacoeconómicos (Gimeno et al., 2006).

Las evaluaciones parciales de una única opción son descripciones y de más se encuadran en otros estudios. Las descripciones aisladas de costes (impacto presupuestario o coste de enfermedad) y de consecuencias (evaluación de calidad de vida) o conjuntas de coste y consecuencias para conocer el coste por unidad utilizada (contabilidad analítica). Los estudios de tipo descripciones no permiten calcular eficiencias, ni son útiles para seleccionar, priorizar o elegir entre estrategias alternativas. En efecto, por ejemplo los estudios de impacto presupuestario tratan de contabilizar los costes que inciden sobre el presupuesto de una agencia, institución, hospital o sistema sanitario, a consecuencia del empleo de una nueva tecnología. Los otros tipos de evaluaciones parciales son principalmente la evaluación de la eficacia, efectividad o utilidad, y el análisis de costes (Gimeno et al., 2006).

\section{Estudios de minimización de costes}

El análisis de minimización de costes se utiliza cuando existen suficientes evidencias que las consecuencias de las alternativas son iguales o equivalentes. Este análisis pretende identificar entre varias alternativas, aquella que genera 
Introducción

menores costes, en la hipótesis de que los resultados de todas ellas son equiparables (Drummond et al., 2005).

Sus principales ventajas son su sencilla aplicación, rapidez de cálculo y de más bajo coste de todos los análisis completos. Puede aplicarse razonablemente para la gestión logística institucional como externalizar servicios o unidades no operativas del hospital. Un ejemplo clínico sería la administración de un aminoglucósido en dosis única diaria o repartido en dos o tres dosis al día, suponiendo que obtenemos la misma efectividad con ambas pautas. Se utiliza para la toma de decisiones en formularios o guías farmacoterapéuticas (Bootman et al., 2004; Sacristán et al., 1995).

Presenta el análisis de minimización de costes como limitaciones, que es infrecuente obtener alternativas terapéuticas con las mismas consecuencias clínicas, en sentido estricto; no es posible hacer comparaciones respecto a otras estrategias o proyectos; y que no se hayan encontrado diferencias evidentes no significa que tales diferencias no existan. Esto sucede frecuentemente al utilizar estudios de no inferioridad de una alternativa frente a otra. Este tipo de análisis puede ser útil para las Comisiones de Farmacia y Terapéutica, ya que se necesitan datos que avalen la no duplicidad dentro de los hospitales (Pinteño et al., 2006).

\section{Estudios coste-efectividad}

Es una forma limitada de evaluación económica que trata de identificar y cuantificar los costes de dos ó más alternativas posibles para alcanzar un objetivo, cuyos resultados vienen habitualmente expresados en efectos (unidades físicas naturales) y cuya comparación se hace en términos de coste por unidad de efecto, utilizando ratios medios o marginales coste-efectividad (Bootman et al., 2004; Sacristán et al., 1995).

Para valorar la relación coste efectividad de los tratamientos, se calculó el ratio coste efectividad incremental $(\mathrm{RCEI})$ de una alternativa frente a la otra que se 
compara. El cálculo se realiza de la siguiente manera, (tomando A y B como alternativas) (Willan y Briggs, 2006):

RCEI $=\Delta \mathrm{C} / \Delta \mathrm{E}=\mathrm{C}_{\mathrm{A}^{-}}-\mathrm{C}_{\mathrm{B}} / \mathrm{E}_{\mathrm{A}}-\mathrm{E}_{\mathrm{B}}$

Siendo: $C_{A}$ : costes de la alternativa $A ; C_{B}$ : costes de la alternativa $A ; E_{A}$ : eficacia o efectividad de la alternativa $A ; E_{B}$ : eficacia o efectividad de la alternativa $A$. Nótese la diferencia de calcular los ratios promedios de cada alternativa y compararlos, es decir calcular CA/EA y CB/EB y compararlos, a diferencia del RCEI que nos informa sobre lo que costaría (en dinero) conseguir una unidad más de eficacia. El signo negativo del ratio incremental puede dar la clave sobre la conveniencia clara de una opción respecto de otra, siendo su valor expresivo de los ahorros logrados por unidad de efecto ganado (Drummond et al., 2005).

Es condición necesaria medir con los resultados de las opciones con la misma unidad natural, como por ejemplo: número de pacientes curados, eventos tromboembólicos evitados, vidas salvadas, días sin dolor, días de hospitalización evitados, años de vida ganados, milímetros de mercurio de tensión arterial reducidos, número de episodios emetógenos, entre otros. Los estudios costeefectividad son los utilizados con más frecuencia debidos a que se ajustan mejor a los resultados de la práctica clínica. Sin embargo, no permiten la comparación entre medicamentos administrados en distintas indicaciones, pero son los más apropiados para medir los resultados en términos de obtener un objetivo específico terapéutico.

Cuando se analizan distintas alternativas una vez colocadas en el plano coste-efectividad se debe definir la frontera eficiente, conocida como la línea quebrada resultante de unir secuencialmente mediante segmentos (partiendo del eje de coordenadas y hacia la derecha) las coordenadas correspondientes a las opciones de inferior coste. Todas las estrategias que quedan por encima de la frontera quedan descartadas. Las innovaciones terapéuticas caen normalmente en el cuadrante dos del plano coste efectividad, es decir son más caras y más efectivas (Willan y Briggs, 2006). 
Introducción

\section{Estudios coste-utilidad}

Las escalas de CVRS pueden ser de varios tipos. Las medidas específicas, orientadas a valorar la efectividad de tratamientos concretos o sobre determinado tipo de pacientes no son útiles a la evaluación económica, pues no cumplen los requisitos anteriormente enumerados. Por las mismas razones se descarta la utilización de medidas genéricas -perfiles SF-36, SIP o NHP-. Las medidas basadas en las preferencias, o medidas de la utilidad sí son válidas para su uso en la evaluación económica, y han dado lugar a la variante del análisis coste-efectividad conocida con el nombre de análisis coste-utilidad (Drummond et al., 2005).

Los estudios coste-utilidad miden los efectos a través de una unidad que integra la calidad y cantidad de vida, AVAC. Los AVACs se obtienen calculando los años de vida ganados ponderándolos con la calidad de vida por técnicas que miden los estados de salud basadas en preferencias o utilidades. Estas técnicas son: escala de categorías, juego estándar, estimación de la magnitud, equivalencia temporal y equivalencia de personas (Drummond et al., 2005). Los AVAC pueden también calcularse mediante la utilización de métodos indirectos usando medidas de escalas de salud agregadas como el EuroQol o el SF-36 (Bootman et al., 2004).

Existen diferentes alternativa a los AVAC menos aceptadas como son los años de vida sanos equivalentes basados en la teoría de la lotería pasados dos veces. Los estudios coste-utilidad son considerados una variante de estudios costeefectividad, siendo su interpretación similar y permiten comparar distintos programas sanitarios entre ellos (Puig-Junoy y Pinto, 2008).

\section{Estudios coste-beneficio}

Los estudios coste-beneficio permiten comparar distintos programas 0 tratamientos, pero tienen la dificultad de asignar valores monetarios a los resultados y no permiten comparar simultáneamente productos con más de una indicación. Inicialmente fueron los más utilizados, pero su baja aceptación por los profesionales sanitarios es debida a que estos estudios asignan un valor monetario a la vida 
humana. En la actualidad es un campo en constante cambio y de investigación. Los métodos y técnicas de valoración monetaria de los efectos sobre el estado de salud son dos: los de preferencia revelada y los de preferencia declarada (Drummond et al., 2005).

Métodos de preferencia revelada: a) Precios Hedónicos: Estimación de la contribución marginal implícita del atributo relacionado con el estado de salud al precio de bien objeto de la transacción (salarios, viviendas, etc.). b) Coste del Viaje: Estimación del valor monetario del coste que los individuos están dispuestos a soportar por acceder a un servicio sanitario en términos de tiempo y coste de desplazamiento (por ejemplo, acceso a una unidad móvil para la realización de mamografías). c) Costes Evitados: Estimación de los costes sanitarios necesarios para mitigar o reducir unos determinados los efectos negativos sobre el estado de salud (lesiones sobre la piel ocasionadas por la reducción de la capa de ozono). d) Aportaciones Voluntarias: Estimación basada en la disposición mostrada por los individuos a contribuir a organizaciones no lucrativas para finalidades relacionadas con la mejora del estado de salud (Puig-Junoy y Pinto, 2008).

Métodos de preferencia declarada: a) Valoración Contingente: Estimación de la disponibilidad a pagar o la disposición a ser compensado/aceptar mediante la simulación de un mercado hipotético con técnicas de encuesta. b) Análisis Conjunto: Estimación de la disponibilidad a pagar a partir de la clasificación ordinal de diferentes alternativas mediante técnicas de encuesta (Puig-Junoy y Pinto, 2008).

En un análisis coste-beneficio pueden calcularse tres índices de decisión diferentes: el valor actual neto, la ratio coste-beneficio (bruta o neta) y la tasa interna de rendimiento. Las reglas de decisión se concretan en: (1) aceptar los programas cuyo valor actual neto (VAN) sea positivo, su ratio coste-beneficio menor que la unidad o su tasa interna de retorno (TIR) superior a la tasa de descuento; (2) elegir entre dos programas aquél que tenga un mayor VAN, una menor ratio costebeneficio o TIR más alta. La utilización de la ratio coste-beneficio para priorizar programas puede dar lugar, no obstante, a decisiones erróneas, por lo que debe extremarse la precaución al respecto (Drummond et al., 2005). 
Introducción

Aunque todas las técnicas de evaluación económica aportan información de interés para la toma de decisiones, es recomendable optar por aquéllas que permiten un mayor grado de comparabilidad de los resultados y una más fácil interpretación de los mismos. Por ello resulta aconsejable llevar a cabo un análisis coste-beneficio cuando se estime oportuno y factible valorar todos los resultados del programa en unidades monetarias, y/o un análisis coste-efectividad que utilice una medida lo más comprehensiva posible de los resultados, preferentemente AVACs es decir, un análisis coste-utilidad-. El análisis coste-consecuencia puede resultar un útil paso intermedio hacia la realización de cualquiera de los dos anteriores. Por último, sólo si los resultados se estiman esencialmente idénticos entre las alternativas examinadas resulta apropiado limitar la evaluación a los costes mediante un análisis de minimización de costes (Drummond et al., 2005; Puig-Junoy y Pinto, 2008).

\subsubsection{Modelización}

Definimos un modelo como la representación abstracta y simplificada de una realidad percibida de una teoría. Los modelos suelen utilizar un lenguaje matemático para construir representaciones esquemáticas de los procesos a fin de proponer explicaciones para estos, prever su evolución y simularlos para facilitar la asunción de decisiones (Auray et al., 1998).

La modelización como método analítico describe los acontecimientos fundamentales que se producen en un tiempo determinado. Los modelos en investigación sanitaria pueden tener forma de árboles de decisión, que resultan adecuados para el estudio de episodios breves, pero que simplifican en exceso y describen de forma pobre aquellas situaciones con alternativas múltiples de ocurrencia y cuyas probabilidades de suceder pueden aparecer con el tiempo (DíezVegas, 2005).

Estas situaciones son habituales por ejemplo en enfermedades crónicas, con respecto a las cuales se repiten constantemente las mismas decisiones. En 
consecuencia, para hacer frente a estas situaciones, se usan métodos más complejos.

Las razones que justifican la utilización de modelos en sanidad se resumen en (Rodríguez-Barrios, 2004):

- Necesidad de generalización de intervenciones sanitarias para favorecer la aplicación de medidas evaluadas desde la investigación a la práctica clínica, y entre los resultados intermedios o variables subrogadas y las variables finales de interés.

- Los estudios y ensayos clínicos muchas veces no recogen información relevante para los estudios de evaluación económica. Los estudios y ensayos clínicos muchas veces no recogen información relevante para los estudios de evaluación económica.

- Extrapolación de datos más allá del periodo del estudio clínico para llegar a resultados finales en salud o más allá de la clínica (relacionado con la calidad de vida y las preferencias del paciente o con el consumo de recursos asociados al manejo de la patología de estudio).

- Síntesis de la información en procesos complejos. En los que se dispone de información de diferentes fuentes o tipos.

- Desarrollo de sistemas que generen decisiones basadas en información contrastada en ausencia de datos concluyentes sobre efectos en salud (mortalidad, etc.).

\subsubsection{Tipos de modelos}

Por definición, un modelo es una visión simplificada de la realidad. Su validez depende de que las hipótesis, o suposiciones, sean razonables, en función de las necesidades de la persona que ha de tomar la decisión y de cuáles sean sus propósitos, y, lo que es más importante, de si las implicaciones que se deriven de sus resultados tienen sentido o carecen de él. Podemos clasificarlos de diferente forma según (Rodríguez-Barrios, 2004): 
1. Su estructura (alternativas excluyentes entre sí).

— Modelos basados en árboles de decisión. El árbol de decisión es un método cuantitativo y sistemático de representación de una situación clínica, que reduce la incertidumbre en la toma de decisiones. En ellos se explicitan las probabilidades de ocurrencia de los sucesos y sus consecuencias. Se caracterizan gráficamente por un conjunto de ramas que representan las diferentes decisiones y nudos. Estos nudos son el punto de origen de cada una de dichas decisiones (nudos de acción y nudos de acontecimiento), o en los que desembocan los resultados finales de la intervención (nudos terminales). En estos modelos, se pueden incorporar parámetros como distribuciones de valores en lugar de como estimaciones puntuales (Díez-Vegas, 2005; Ríos-Insua et al., 2002).

- Modelos de Markov, en los que los riesgos asociados a los diferentes estados de salud se asocian a probabilidades que pueden ser cambiantes en el tiempo (procesos de Markov), o constantes (cadenas de Markov). En el ámbito sanitario, estos modelos están cobrando una importancia creciente.

—Otros: Simulaciones complejas basadas en modelos informáticos, modelos de supervivencia, etc.

2. Si su naturaleza es probabilística (modelos excluyentes entre sí) o no, los modelos se clasifican en (Rubio-Terrés, 2000):

- Modelos determinísticos. Las variables de interés de los estudios (efectos de tratamiento, probabilidades de supervivencia, individuos en cada estado de salud), se introducen y computan directamente a través de fórmulas algebraicas, sin utilizar técnicas de simulación, dado que se suponen no sujetas a incertidumbre.

-Modelos estocásticos. Son modelos probabilísticos que usan la incertidumbre como parte del cálculo, por lo que se emplean técnicas de aleatorización para simular las probabilidades de los eventos que podrían generarse por efecto del azar. El modelo estocástico más frecuentemente utilizado en evaluación económica es el de Markov. 
3. Las técnicas cuantitativas que utilice, la evaluación económica en sanidad, utiliza métodos de otras disciplinas; según la procedencia de estas técnicas, podemos clasificarlos en (Dummond et al., 2005):

- Modelos de análisis de decisiones. Comenzaron a aplicarse en sanidad por Weinstein y Fineberg en 1980 (Weinstein, 1980). Aportan una herramienta epidemiológica clínica cuantitativa a los clínicos y pacientes con el fin de cuantificar riesgos, beneficios utilidades y costes; ligando opciones terapéuticas alternativas para pacientes individuales. Utilizan la forma de árboles de decisión, y sus inputs clínicos se utilizan en forma de probabilidades de decisión clínica. Estos modelos pueden ser fáciles e intuitivos sin llegar a mostrar gran complejidad (infección aguda, estrategia de vacunación, etc.) (Rodríguez-Artalejo et al., 1990; Ríos-Insua et al., 2002; Díez-Vegas, 2005).

- Modelos de extrapolación. Como se ha comentado, los ensayos clínicos, presentan frecuentemente limitaciones en cuanto a la duración del seguimiento, sin embargo, las necesidades de la evaluación económica requieren de mediciones a más largo plazo, por lo que se emplean modelos estadísticos más completos.

- Modelos de Markov. Aplicados a enfermedades, tratamientos o situaciones sanitarias que presentan recurrencia de estados de salud o que por su carácter crónico, presentan muchos estados diferentes. Podrían utilizar como forma de representación árboles de decisión, pero la complejidad y el elevado número de estados de salud modelizados en ellos, hace que se prefiera representarlos mediante diagramas de estado (infección HIV).

-Epidemiológicos: existen áreas de la enfermedad donde los resultados finales de la progresión de la patología pueden tardar años en producirse, pero los ensayos clínicos, normalmente evalúan la eficacia en términos de punto final intermedio (variables subrogadas), por ejemplo en las patologías cardiovasculares (hipertensión, hipercolesterolemia, etc.). Esto plantea problemas a la hora de realizarlos en análisis económicos si se decide vincular el punto final clínicobiológico a los resultados finales en salud. En dichos casos se usan modelizaciones 
Introducción

epidemiológicas para vincular los resultados intermedios y finales. Se suelen emplear ecuaciones de regresión como forma de análisis, usándose los coeficientes de regresión como inputs clínicos. Estos modelos combinan frecuentemente ensayos clínicos aleatorios y datos epidemiológicos como por ejemplo la predicción de la mortalidad cardiovascular a través de variables subrogadas (hipertensión, triglicéridos y colesterol en sangre, etc.).

Esta última clasificación no es excluyente, es decir, podemos por ejemplo desarrollar un proceso de Markov, que incorpore datos de un modelo epidemiológico de progresión de la enfermedad a estudiar (Rodríguez-Barrios, 2004).

El uso de técnicas de modelización viene a llenar los espacios no cubiertos por los ensayos clínicos, tanto por su corta extensión temporal, como por la ausencia de datos clínicos de resultados finales (no subrogados), de la patología estudiada, así como la necesidad de integrar la información económica en un entorno de asistencia sanitaria de recursos limitados. Todo ello justifica el uso de los modelos como apoyo a la toma de decisiones, o incluso facilitar a decisores y descriptores información sobre diferentes opciones de tratamiento y sus posibles resultados (Robert et al., 1983).

Por lo tanto, los modelos ayudan a tomar decisiones, a integrar decisiones clínicas en la práctica, a apoyar nuevas normativas o a documentar la selección farmacoterapéutica más eficientemente (DiMasi et al., 2001).

Los modelos de Markov son un tipo de modelo matemático de uso cada vez más extendido como herramienta de apoyo en la evaluación económica, ya que su versatilidad permite usarlos en patologías crónicas o en aquellas situaciones o problemas de salud que presentan recurrencias (exacerbaciones, agudizaciones de procesos crónicos). Ambas situaciones son las que consumen más cantidad de recursos en los sistemas sanitarios, por lo que resulta de gran interés para los decisores (Rodríguez-Barrios, 2004). 
La transparencia y la calidad del modelo empleado deben asegurarse a través de su validez conceptual, la solvencia de sus fuentes y un diseño según las revisiones aceptadas internacionalmente (Khan y Miller, 1999; Rodríguez-Barrios, 2004). Su reproducibilidad, así como una validación transparente (Kuntz y Weinstein, 2001), ha de ser una exigencia fundamental, así como la realización de un completo análisis de incertidumbre de las variables críticas del modelo (justificando su selección y los rangos decididos para el análisis). Además, la construcción, de escenarios cuantitativos argumentados y utilizables en la toma de decisiones para los responsables sanitarios, debe ser también un elemento consustancial a los modelos, ya que aumentará su aplicabilidad. Por último, el incremento de la tendencia a requerir evaluaciones económicas como parte de los procesos de aprobación y reembolso de nuevos fármacos, incrementará el uso de modelos en este ámbito (Lang et al., 2003; Siebert, 2003; Rodríguez-Barrios, 2004).

\subsubsection{Análisis de la incertidumbre}

Los estudios farmacoeconómicos presentan una serie de sesgos que invalidan sus resultados y crean incertidumbre en vez de disminuirla, y son principalmente: de patrocinio, metodológicos y de costes. La incertidumbre depende de la variabilidad de los parámetros, de la extrapolación o generalización de los resultados y de los modelos analíticos empleados. El análisis de la incertidumbre en farmacoeconomía, llevada a cabo principalmente en los análisis coste-efectividad, puede hacerse utilizando dos grupos de técnicas: mediante análisis de sensibilidad cualitativos y análisis estadísticos de la incertidumbre (Rubio-Terrés et al., 2004; Díez-Vegas, 2005).

El análisis de sensibilidad se aplica cuando existe incertidumbre respecto de los valores numéricos reales de los parámetros que influyen en el acaecimiento de un suceso, intentando conocer en qué medida los resultados y las decisiones posteriores pueden verse afectadas. Puede ser determinístico (univariante, multivariante, de escenarios, de umbral) o probabilístico (Rubio-Terrés et al., 2004). 
El análisis de sensibilidad univariante examina, de forma aislada, el impacto (de cada una de las variables sometidas a incertidumbre) sobre los resultados y las conclusiones del estudio, modificando sus valores dentro de rangos de variación razonables. Aunque carece de sustento teórico ya que los parámetros no son independientes, permite identificar los parámetros más influyentes en los resultados del análisis así como valorar el grado de robustez del mismo. El análisis de sensibilidad multivariante, principalmente el bivariante y el trivariante ya que cada vez es más difícil su interpretación (Puig-Junoy y Pinto, 2008).

En el análisis de sensibilidad de escenarios se contrastan los efectos sobre los resultados finales de diferentes escenarios, cada uno de los cuales afecta a la fijación de valores de varios parámetros al mismo tiempo. El análisis de escenarios extremos combina los valores que producen la mejor y la peor razón costeefectividad. El análisis de umbral identifica los valores críticos del parámetro por encima o por debajo de los cuales las conclusiones de la evaluación final cambia (Puig-Junoy y Pinto, 2008). Los análisis de sensibilidad pueden presentarse gráficamente como diagramas de tornado o de araña (Díez Vegas, 2005).

Otra manera de analizar la incertidumbre es estadísticamente. En efecto, conocer la distribución estadística del cociente entre dos parámetros es un reto teórico. Para el cálculo del intervalo de confianza del ratio coste-efectividad se utilizan diversos métodos paramétricos (como los cuasi IC, la expansión de series de Taylor, las elipses de confianza o el método de Fieller) o los métodos de muestreo repetitivo no paramétricos. De todos estos métodos parecen ser preferibles el de Fieller y el remuestreo. Distinto enfoque del problema es la utilización de análisis de sensibilidad probabilísticos con la ayuda de la simulación de Monte Carlo. Actualmente parece el método más utilizado por su facilidad de uso y permite el análisis de subgrupos de pacientes (Rubio-Terrés et al., 2004).

Pocos estudios realizan en el ámbito sanitario la modificación de la estructura del modelo utilizado principalmente por: su compleja aplicabilidad tanto en trabajo como en conocimientos metodológicos, la reducción de la incertidumbre es insatisfactoria y por la falta de validez clínica de cada modelo utilizado. 
Introducción

\subsection{Estudios de calidad de vida relacionada con}

\section{la salud}

La vida tiene dos dimensiones: calidad y cantidad (Ware, 1995). La salud es uno de los doce dominios de la vida considerados por Campbell cuando evaluamos la calidad de vida total de las personas; el resto son: la nación, la comunidad, la autoestima, la vida familiar, la relación con los amigos, la economía, la educación, el hogar, el pueblo o barrio donde vivimos, el trabajo y si estamos o no casados (Campbell, 1981). Por tanto, debe considerarse CVRS como un término diferente a la calidad de vida.

La CVRS es el valor asignado a la duración de la vida modificado por la disminución, estado funcional, percepciones y oportunidades sociales debido a una enfermedad, accidente, tratamiento o política. Los pacientes son la principal fuente de información en los estudios de CVRS, de ahí que muchos investigadores utilicen también para referirse a estos estudios como resultados declarados por los pacientes, $\mathrm{PRO}=$ patient-reported outcomes.

El impacto de una enfermedad y su tratamiento a menudo afecta a más de una función biológica. Anormalidades y desequilibrios en la función biológica puede impactar en importantes aspectos de la vida diaria del paciente, incluyendo su bienestar general y su comportamiento funcional. Este impacto es comparable a la propagación de la onda debida al impacto de una piedra en la superficie de una charca o estanque (Ware, 1995; Bungay et al., 2005).

La CVRS es un concepto complejo con múltiples dimensiones. Estos aspectos, conocidos como dominios, pueden incluir desde síntomas que tienen los pacientes hasta su función sexual o bienestar general. Como ejemplo los cuatro dominios que mide el SF-36, el cuestionario más utilizado a la hora de valorar la CVRS son la función física, la función de relaciones sociales y del trabajo, la salud mental y la percepción global de la salud (Ware y Sherbourne, 1992). 
Aristóteles fue el primer filosofo que habló de "la buena vida y del hacerlo bien", que significa sentirse feliz, por lo que no solo se fijaba en la cantidad sino también en la calidad de la vida de las personas. El primer cuestionario utilizado ampliamente para su uso clínico fue la Escala de Karnofsky utilizado para valorar las limitaciones físicas de los pacientes gravemente enfermos y especialmente en aquellos aquejados de cáncer; siendo una simple medición de 0 a 100 y que en el transcurrir de los años ha degenerado en otras escalas que miden la habilidad funcional, el funcionamiento físico y las actividades de la vida diaria como el Índice de Barthel (Mahoney y Barthel, 1965), muy extendido en para su uso en la población geriátrica. Aunque estos cuestionarios se describen dentro de los instrumentos de CVRS, éstos solamente capturan un aspecto de la misma CV y se obtiene una inadecuada representación de bienestar global del paciente y de su CVRS (Patrick y Ericsson, 1993; Fayers y Machin, 2007; Carrera-Hueso, 1997a; Walters, 2009).

Estos cuestionarios fueron el punto de partida para el desarrollo de los cuestionarios que actualmente se utilizan en investigación y en clínica. En efecto, estos cuestionarios modernos aparecieron en los años 70 y están basados en la construcción de escalas permiten medir no sólo un aspecto o dominio de la CVRS. Estos estados de salud cuantificados, llamados perfiles de salud, se utilizan para la evaluación general de la salud de los pacientes. Entre estos cuestionarios podemos citar por su amplia utilización: SIP-Sickness Impact Profile (Bergner et al., 1976) y el NHP-Nottingham Health Profile (Hunt et al., 1985).

Posteriormente hubo una aproximación para que se pudiesen auto-administrar los cuestionarios o por medio de la escala visual análoga que permiten la no intervención de un entrevistador avezado y son muy intuitivos para los pacientes. Un ejemplo de este tipo de escalas están contenidas en el cuestionario EuroQol-5D (EuroQol, 1990).

Los más recientes instrumentos, como el SF36 (Ware y Sherbourne, 1992), hacen hincapié en medir los aspectos subjetivos de la CVRS, tales como los emocionales, sociales y la función cognitiva; pero sin descuidar la medición de los aspectos físicos y de discapacidad de la misma. Otros cuestionarios hacen más 
profusión en incorporar los valores personales de los pacientes, por lo que las opiniones directas de los pacientes sobre todos los aspectos de su vida reflejarán mejor su CV.

En la actualidad existe un gran interés sobre este campo como puede comprobarse al haberse producido un incremento exponencial de los estudios de CVRS realizados, yendo en la base de datos de Medline (PubMed de la US National Library of Medicine) [palabras clave: "health related quality of life", fecha de realización de la búsqueda: 20 de abril de 2011] desde 86 publicaciones en 1990, hasta 896 y 3622 en los años 2000 y 2010 respectivamente, con un total de artículos acumulado de 27.120 en la fecha de búsqueda.

\subsubsection{Cuestionarios genéricos}

Algunos instrumentos son de uso general, independientemente de la enfermedad o condición del paciente, e incluso se pueden utilizar para personas sanas. En efecto, muchos de estos cuestionarios fueron desarrollados para aplicarlos en encuestas de poblaciones, aunque posteriormente se aplicasen en ensayos clínicos y en la práctica clínica diaria (Fayers y Machin, 2007; Walters, 2009).

Existen muchos instrumentos que miden la discapacidad física, alguno de ellos lo hacen específicamente sobre su repercusión en las actividades diarias de los pacientes como el Índice de Barthel (Mahoney y Barthel, 1965). Aunque comúnmente son descritos como escalas de CVRS, estos instrumentos son llamados mejor como medidas del estado de salud porque se enfocan sobre los síntomas físicos únicamente. Ellos se centran o enfatizan más sobre la medición de la salud general y hacen implícita la asunción de peor salud indica peor CVRS. Una de sus debilidades acerca de esta manera de valoración es que diferentes pacientes pueden reaccionar de manera diferente a niveles similares de discapacidad. Muchos de los cuestionarios iniciales fueron diseñados sobre estos axiomas o asunciones, entre los que mencionaremos el SIP (Bergner et al., 1976) y el NHP (Hunt et al., 1985). 
Introducción

Pocos de los instrumentos desarrollados inicialmente tuvieron en cuenta los aspectos o dominios no físicos de la CVRS, es decir no contemplaban escalas que midiesen su aspecto emocional, social o existencial. Sin embargo, los nuevos instrumentos ya han tenido presente este tipo de dominios en sus escalas, enfatizando fuertemente sobre los aspectos subjetivos de la CVRS y además contemplando una o más preguntas explícitas o directas sobre la CVRS total. El cuestionario por excelencia de estos nuevos es el SF-36 (Ware y Sherbourne, 1992).

Más recientemente se han desarrollado instrumentos más breves al objeto de aumentar su aceptación por parte de los pacientes y profesionales sanitarios, es decir con menos preguntas, que facilitan su cumplimentación por parte de los pacientes que incluso hacen menos énfasis en el funcionamiento físico de los mismos, como por ejemplo el EQ-5D (EuroQol, 1990) o el SEIQol (Hickey et al., 1996), que sirven para obtener utilidades a la hora de realizar estudios costeutilidad.

Los instrumentos genéricos evalúan todos los aspectos relevantes de la salud y no están influenciados por el tipo de enfermedad, raza, edades, etc. Son multidimensionales, es decir, están diseñados para aplicarlos en diferentes enfermedades, intervenciones médicas y pueden utilizarse en una amplía gama de poblaciones. Su principal limitación es intercultural, porque requieren de su adaptación al menos lingüística para trasladarlos a otros países de sonde fueron originados (Fayers y Machin, 2007; Walters, 2009). Los principales instrumentos se encuentran adaptados para la población española.

Los cuestionarios genéricos intentan cubrir un amplio rango de condiciones y tienen la ventaja de que sus resultados o puntuaciones permiten comparar varias enfermedades entre sí o incluso compararlos con las puntuaciones obtenidas en la población general. Estos instrumentos sin embargo fallan a la hora de centrarse en cuestiones o características específicas de una enfermedad y a menudo tienen poca sensibilidad para detectar diferencias al comparar tratamientos entre grupos de pacientes o mejoras en la CVRS de un paciente concreto tras alguna intervención 
Introducción

sanitaria; para ello se utilizan los instrumentos específicos (Fayers y Machin, 2007; Walters, 2009).

\subsubsection{Cuestionarios específicos}

Los cuestionarios específicos asumen que cada condición médica tiene un específico resultado y además deben medirse las funciones afectadas por esa condición. Estos cuestionarios valoran solamente aquellos aspectos considerados importantes en una enfermedad o síntomas relevantes o estados mentales de un determinado grupo de pacientes (Fayers y Machin, 2007; Walters, 2009).

Como ya hemos comentado los cuestionarios específicos de una enfermedad o condición permiten encontrar diferencias entre grupos de pacientes que son tratados con distintos tratamientos, ya que son más sensibles para detectar cambios en la CVRS.

Además de su indiscutible utilidad en ensayos clínicos, por la propiedad detectar cambios que poseen, muchos autores los recomiendan como herramienta para el seguimiento de los pacientes en la práctica clínica diaria. Su principal inconveniente es que solamente pueden aplicarse en los casos para los que fueron diseñados y no permiten comparaciones entre distintas enfermedades o condiciones, ni tampoco con resultados obtenidos en una encuesta de población (Fayers y Machin, 2007; Walters, 2009).

Diferentes áreas han sido investigadas con instrumentos específicos como el funcionamiento sexual y emocional, náuseas y vómitos, ansiedad y depresión, asma, rinitis y epilepsia. Los cuestionarios más relevantes utilizados son. entre otros: EORTC QLQ-C30 ó European Organization for Research and Treatment of Cancer (Aaronson et al., 1993), FACT-G ó Functional Assessment of Cancer TherapyGeneral (Cella et al., 1993), RSCL ó Rotterdam Symptom Checklist (de Haes y Stiggelbout, 1996), QOLIE-89 ó Quality of Life in Epilepsy (Devinsky et al., 1995), y el PAQLQ ó Paediatric Asthma of Life Questionanaire (Juniper et al., 1996). 


\subsubsection{Cuestionarios para aspectos específicos de la CVRS}

Muchas veces lo que interesa no es tanto medir la CVRS en su globalidad por medio de una única puntuación sino medir un aspecto o componente más concreto y con mayor detalle de la CVRS, independientemente de la enfermedad o condición del paciente, y para ello se diseñaron cuestionarios específicos que evalúan determinados dominios (Fayers y Machin, 2007; Walters, 2009).

Estos dominios que suelen explorarse con mayor profundidad son la ansiedad y depresión, el funcionamiento físico, la fatiga, el dolor y la ansiedad y depresión; los cuales son muy importantes las CVRS de los pacientes con enfermedades crónicas o avanzadas. Debemos mencionar que este tipo de cuestionarios normalmente se utilizan junto con un cuestionario más global o general como los descritos en los apartados anteriores.

Entre los cuestionarios más destacados podemos mencionar: HADS ó Hospital Anxiety and Depression Scales (Zigmond y Snaith, 1983), MPQ ó McGill Pain Questionnaire (Melzack, 1975), MFI ó Multidimensional Fatigue Inventory (Smets et al., 1995) y el Índice de Barthel (Mahoney y Barthel, 1965). 
OBJETIVOS 
El principal objetivo principal del presente trabajo es:

$\checkmark$ Utilizar la información proporcionada por los estudios de resultados en salud tanto como herramienta en la toma de decisiones farmacoterapéuticas como para la justificación de servicios clínicos.

Otros objetivos también se plantean son:

> Mostrar la incorporación de los estudios farmacoeconómicos en el ámbito hospitalario.

> Aplicar los estudios farmacoeconómicos en situaciones clínicas reales adaptados a nuestro entorno.

> Utilizar el análisis de la incertidumbre para garantizar la robustez de los resultados obtenidos.

> Realizar un análisis de sensibilidad estructural además del determinístico de los parámetros.

> Mostrar la utilidad de medir la calidad de vida relacionada con la salud en la atención sanitaria a los pacientes. 
RESULTADOS

$Y$

DISCUSIÓN 


\section{Capítulo 1}

Carrera-Hueso FJ. Análisis de los resultados sanitarios y su aplicación. Rev Med Univ Navarra 1997; 41: 31-38. 


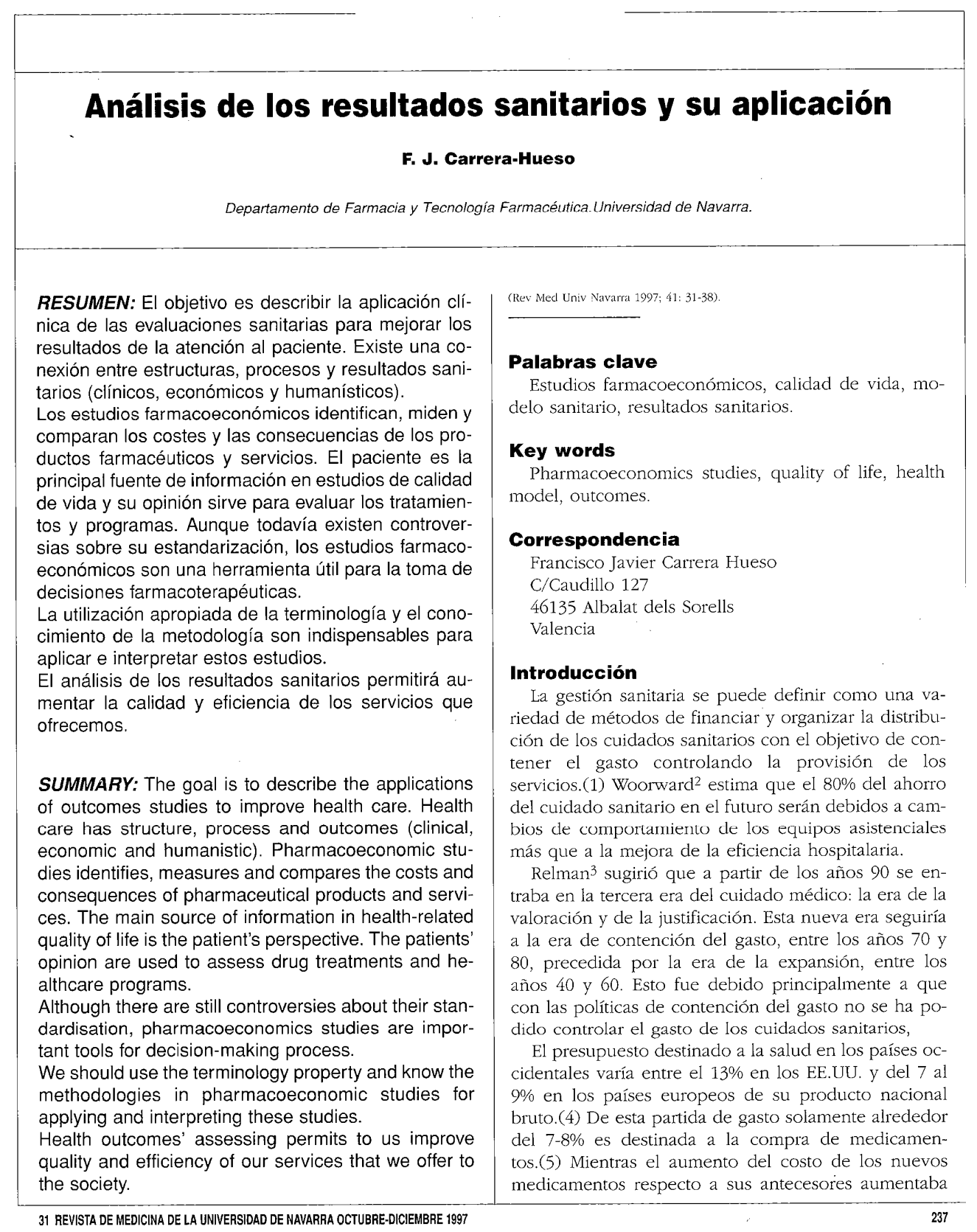


Resultados y discusión

continuamente no quedaba claro que éstos también mejorasen la esperanza o calidad de vida de los pacientes. (6). El costo de los medicamentos ha ido cre ciendo progresivamente en la última década dos o tres veces por encima de la inflación y las previsiones estiman que los nuevos medicamentos y tecnologías incrementarán el gasto sanitario en un 2.8\%.(5)

A pesar de que los medicamentos constituyen sólo una pequeña parte del gasto sanitario, éstos todavía merecen la atención de las Administraciones Sanitarias por al menos estas cuatro razones: el gasto sanitario continua creciendo, los medicamentos son considerados como productos más que como servicios, algunos productos farmacéuticos aportan un dudoso beneficio y existe una conciencia generalizada de que los nuevos productos biotecnológicos aumentarăn el gasto farmacéutico hasta niveles sin precedentes.(7).

El objetivo del presente trabajo es describir la aplicación clínica de las evaluaciones sanitarias para mejorar los resultados de la atención al paciente.

\section{Modelos sanitario}

Tradicionalmente la medicina estaba más preocupada por sanar o paliar el efecto de las enfermedades que en mejorar el bienestar social. Para ello utilizaba tres indicadores para evaluar sus resultados: mortalidad, morbilidad y el coste. Estos indicadores también servían para la toma de decisiones farmacoterapéuticas y su posterior seguimiento.(6).

Si tenemos en cuenta la definición de salud dada por la Organización Mundial de la Salud: "estado com-

Figura 1

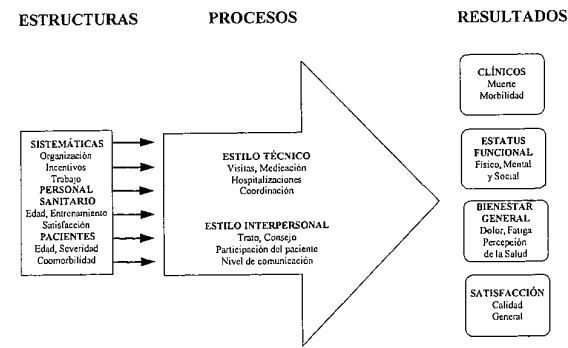

Transcurso de la enfermedad.

32 REVISTA DE MEDICINA DE LA UNIVERSIDAD DE NAVARRA OCTUBRE-DICIEMBRE 1997
Figura 2

pleto de bienestar físico, mental y social, no solamen te la ausencia de la enfermedad",(8) estos indicadores no son útiles ni para monitorizar el resultado de nuestras actuaciones ni para disminuir el coste total de los tratamientos farmacológicos

En 1988, Ellwood ${ }^{9}$ describió el modelo de la gestión de los resultados de los cuidados sanitarios y propuso medir sistemáticamente en la práctica clínica diaria el grado de funcionamiento y de bienestar de los pacientes.

Según Donabedian ${ }^{10}$ la calidad de los servicios sanitarios depende de la técnica, considerada como el conjunto de habilidades y conocimientos, y de las relaciones interpersonales, contemplándose la opinión de los pacientes acerca de sus tratamientos. Este autor considera la existencia de una conexión entre estructuras, procesos y resultados sanitarios,(10-12) y que solamente podemos aumentar la calidad de los servicios sanitarios si mejoramos los procesos a través de programas de garantía de calidad o mejorando los resultados sanitarios a través de los estudios farmacoeconómicos y de calidad de vida.(10). Estos resultados sanitarios son entendidos como los efectos de los cuidados sanitarios sobre los pacientes y/o poblaciones (Figura 1).(12)

Kozma et al.(13), diseñaron un modelo gráfico para comprender el complicado proceso de la toma de decisiones farmacoterapéuticas en el que no solo se miden los resultados clínicos en el tratamiento de los pacientes sino también los resultados económicos y humanísticos del mismo.(11,12). El modelo es descri-

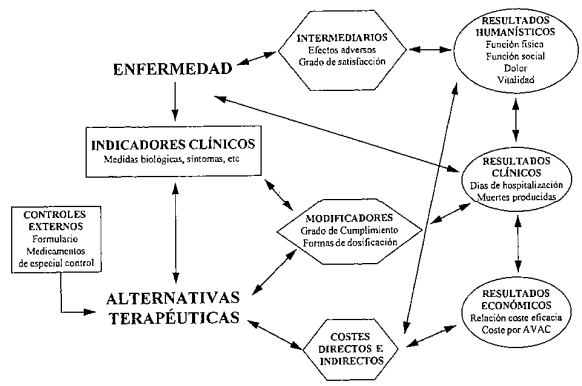

Modelo de resultados. 
to en la Figura 2. Los resultados clínicos son los derivados de una enfermedad o tratamiento (morbilidad y mortalidad); los económicos son aquellos que comparan los costes y las consecuencias de las distintas alternativas; y los humanísticos son las consecuencias de la enfermedad sobre el estatus funcional del paciente o medidas sobre la calidad de vida en varias dimensiones.

El modelo también contempla la existencia de una serie de intermediarios o modificadores del tratamiento que pueden afectar a los resultados del mismo. La importancia de estos intermediarios es en ocasiones muy alta. Así por ejemplo el incumplimiento de los tratamientos por los pacientes es alrededor del $25 \%$ y supone un coste directo anual en los EE.UU. de 50 billones de dólares (b\$), se piensa que la misma cantidad supondrían los costes indirectos de estos tratamientos.(14). Recientemente Johnson y Bootman ${ }^{15}$ han estimado, mediante el diseño de un modelo farmacoeconómico, el coste de la morbilidad y mortalidad debido a los problemas relacionados con la utilización de medicamentos en los EE.UU. El coste calculado asciende a 76.6 b\$, el cual varía con el análisis de sensibilidad entre 30.1 y 136.8 b\$, imputándose a las hospitalizaciones el mayor gasto provocado por la utilización de medicamentos en 47 b\$. Los autores concluyen que los problemas relacionados con la utilización de medicamentos pueden considerarse como una "enfermedad de comportamientos", la cual puede prevenirse con la utilización de servicios y controles sanitarios.

\section{Tipo de estudios}

Como indicabamos anteriormente solamente podemos mejorar los resultados por medio de programas de garantía de calidad de los procesos o incidiendo directamente sobre los mismos por medio de estudios farmacoeconómicos y de calidad de vida.(10).

La industria farmacéutica aplica estos estudios desde la última década, invirtiendo muchos recursos en el desarrollo de los mismos. La media de estudios por empresa farmacéutica ascendió del 1.7 en 1988 al 23.7 en 1994.(16). También el número de estudios clínicos publicados que incorporan variables económicas de la salud ha aumentado exponencialmente. El porcentaje de estos estudios respecto al total fue para los años 1988 y 1994 del 2.6 y del $28 \%$ respectivamente.(16).

Grossman argumenta que las razones para valorar los resultados sanitarios son: definir, evaluar y mejorar la efectividad del servicio prestado; controlar los cos- tes de los servicios superfluos o inapropiados y documentar el valor del cuidado sanitario.(6).

\section{Estudios farmacoeconómicos}

Los estudios farmacoeconómicos (FE) identifican, miden y comparan los costes (ej.: recursos consumidos) y las consecuencias (clínicas, económicas y humanísticas) de los productos farmacéuticos y servicios. Estos estudios incluyen además de las evaluaciones farmaeconómicas, como los estudios de coste de una enfermedad y los análisis de decisión, las evaluaciones humanísticas, como las preferencias y satisfacción de los pacientes, y los estudios de calidad de vida relacionada con la salud (CVRS).(7). Ia farmacoeconomía se engloba dentro de la evaluación económica de tecnologías sanitarias. Los estudios de CVRS se describirán en una sección más adelante.

En la Tabla 1 se describen los diferentes tipos de análisis que se pueden realizar. $(7,17,18)$. Los análisis se diferencian entre sí por como miden los efectos de los tratamientos, ya que todos ellos miden los costes en unidades monetarias.

Los estudios de minimización de costes no miden los resultados ya que suponen que los efectos de las alternativas son equivalentes basándose en publicaciones previas sobre eficacia. Estos estudios son los más utilizados en la toma de decisiones de formularios o guías farmacoterapéuticas.

Los estudios coste-efectividad son los más frecuentemente utilizados debido a que se ajustan mejor a los resultados de la práctica clínica diaria.(19). No se pueden utilizar cuando comparamos simultáneamente productos con más de una indicación

Los estudios coste-utilidad miden a través de una unidad que integra la cantidad y calidad de vida, años de vida ajustados por calidad (AVAC) o años de vida sanos equivalentes.(20). Esto se realiza calculando los años de vida ganados ponderándolos con la calidad de vida por técnicas que miden los estados de salud basadas en preferencias o utilidades. Los estados de salud obtenidos pueden agregarse obteniendo una puntuación global, a partir de la cual se calculan los AVAC para los estudios coste-utilidad. Los AVAC también pueden calcularse por métodos indirectos utilizando las medidas de utilidad o escalas de salud, las cuales se describirán posteriormente.

Diversos estudios muestran que tanto en la literatura médica(21), como en la farmacéutica(22), existen interpretaciones erróneas de la terminología así como de la metodología utilizada en los estudios farmacoeconómicos. Por lo que debemos de ser bastante críticos al valorar este tipo de estudios antes de trasladar los 


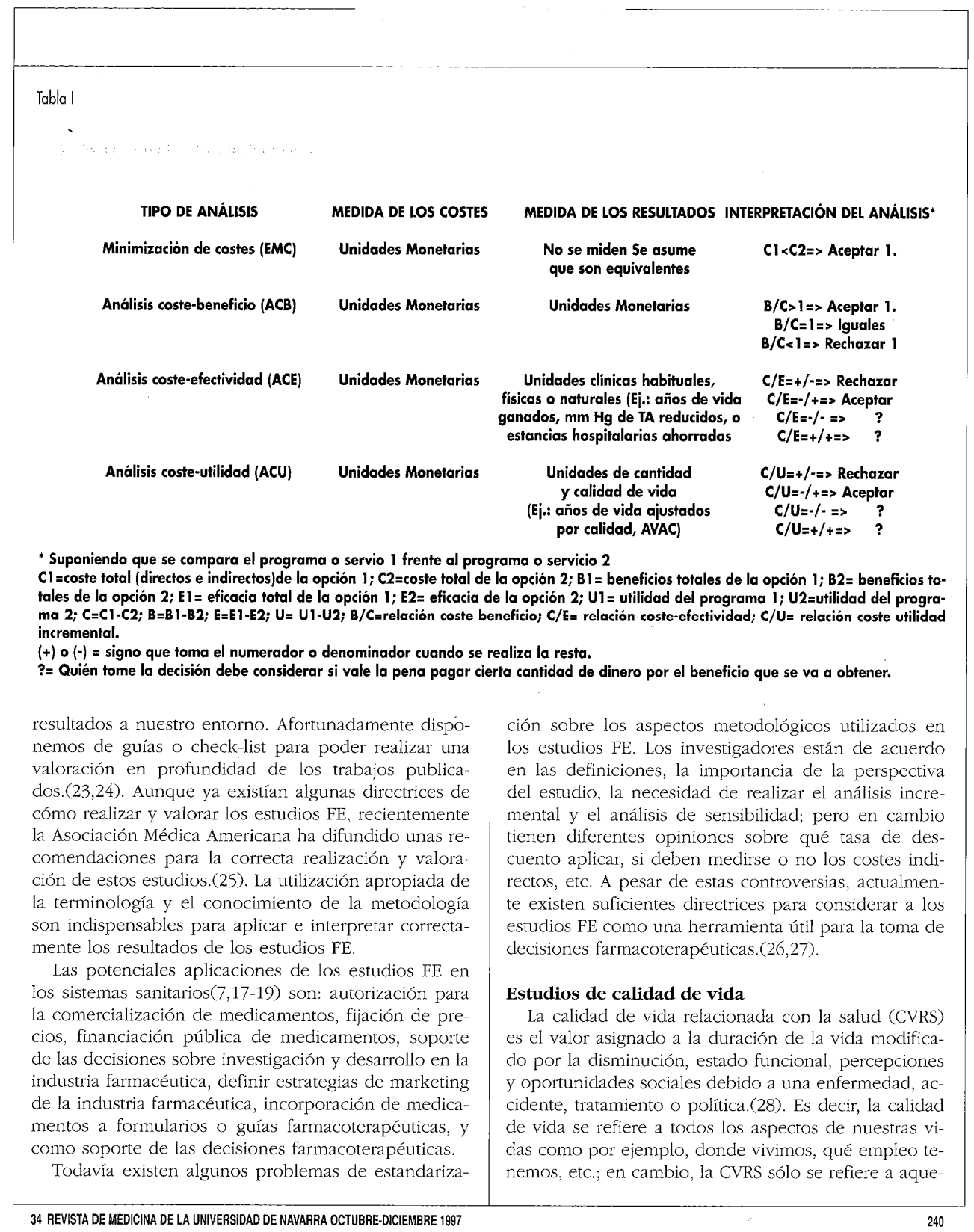




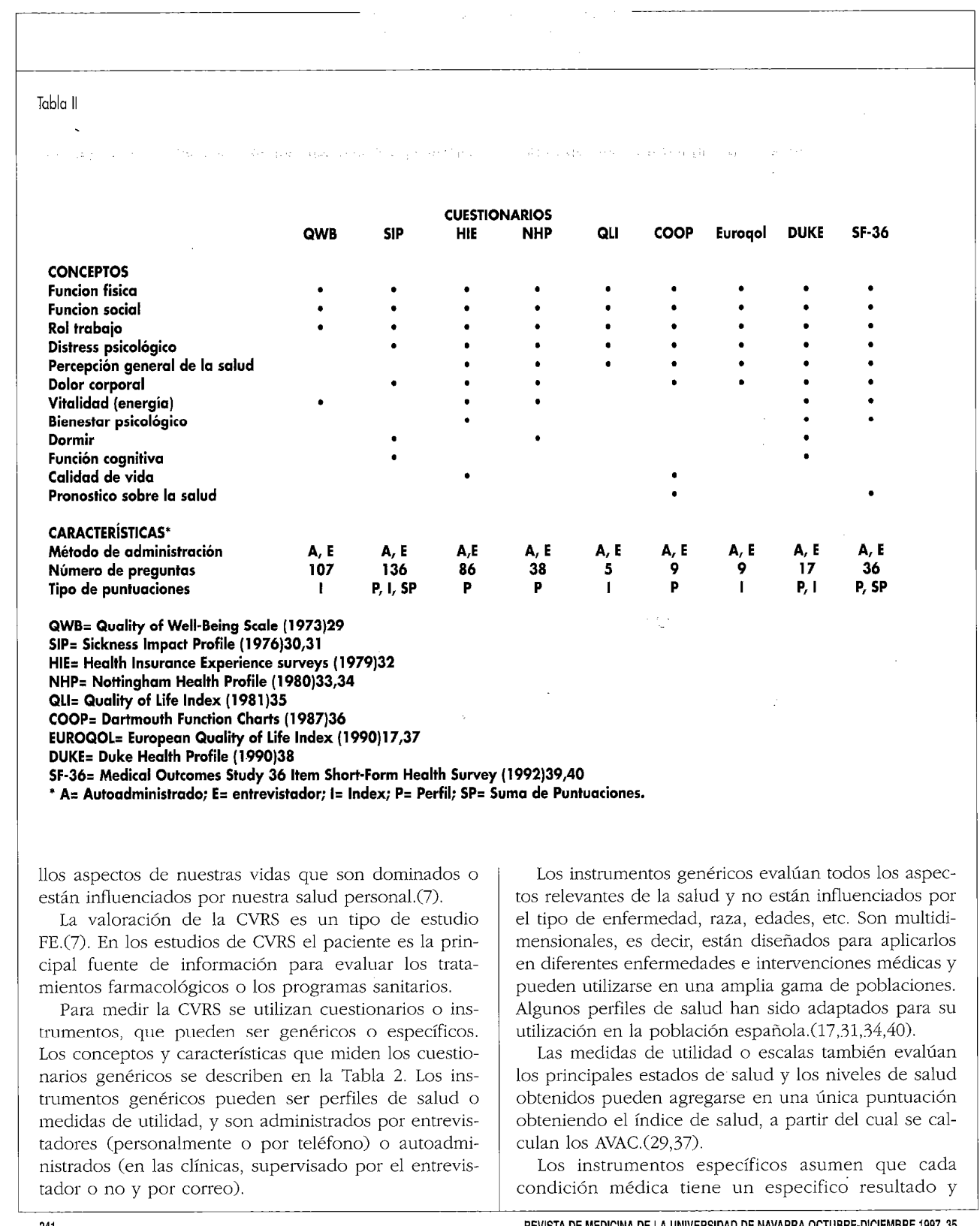


Resultados y discusión

además deben medirse las funciones afectadas por esa condición. Estos cuestionarios valoran sólo aquellos aspectos importantes de una enfermedad o condición, y se han utilizado en artritis reumatoide, cáncer, hipertensión, etc.(41-43). Su principal inconveniente es que únicamente pueden aplicarse en los casos para los que fueron diseñados y no permiten comparaciones entre enfermedades, sin embargo tienen la ventaja de presentar una alta sensibilidad a los cambios clínicos. $(18,44)$

Para medir la CVRS en los pacientes con tratamientos farmacológicos, Aaronson44 propuso la utilización de un test genérico y otro específico, de esta manera obtendríamos con el específico una información más detallada y sensible a los cambios clínicos, y con el genérico podriamos comparar los resultados con otras enfermedades.

Los instrumentos deben de cumplir una serie de requisitos antes de ser utilizados como herramientas para medir y valorar la CVRS de los pacientes. Estos son45: fáciles de usar, interpretar y administrar; aplicables a diferentes poblaciones; ser fiables, validos y prácticos; deben incluir aspectos del bienestar de los pacientes; tener un amplio rango de medidas y ser fácilmente entendibles.

Los estudios de CVRS proporcionan una información acerca de las preferencias de los pacientes, de su bienestar y satisfacción con el cuidado sanitario, la cual puede ser utilizada en la toma de decisiones farmacoterapéuticas

A diferencia de los estudios FE, los distintos grupos de investigadores han ido desarrollando sus propios cuestionarios, lo que impide la comparación con otros estudios publicados.(45). Desgraciadamente no todas los cuestionarios han demostrado una validez y fiabilidad contrastada. Otras cuestiones metodológicas que deben considerarse en la valoración de los estudios de CVRS son qué dimensiones deben medirse y cuales no, la relativa importancia de las dimensiones medidas en el estado global de CVRS, qué instrumento debe utilizarse, qué es más útil medir el índice o los perfiles.

La utilización clínica de los resultados de la CVRS es incipiente y se han aplicado principalmente en el tratamiento y monitorización de enfermedades crónicas como la hipertensión, hemodiálisis, cáncer, epilepsia, etc; $(12,41-43,46)$ y en algunos ensayos clínicos de medicamentos. Queda por delicada el alcance que pueda tener la utilización de estos estudios en el quehacer diario de los profesionales sanitarios y su influencia en el sistema sanitario.

\section{Futuro}

En una sociedad donde sus recursos son limitados, la eficiente distribución de los mismos es la principal misión encomendada a los responsables sanitarios. Para llevar a cabo de manera eficaz este objetivo, en el análisis de los resultados de las diferentes alternativas terapéuticas se deben considerar no solamente los resultados clínicos sino también los económicos y humanísticos; lo cual redundará en una mejor y más acertada toma de decisiones sanitarias.

Los métodos de contención del gasto irán dirigidos a:47 disminuir las admisiones hospitalarias, acortar los días de estancia, utilizar menos tratamientos intensivos (cuidados intensivos, intervenciones quirúrgicas, etc.) y aumentar la utilización de los servicios ambulatorios tanto quirúrgicos como médicos. Esto significa que los cuidados sanitarios se dirigirán más a la prevención de enfermedades que a su tratamiento. Más recursos irán dirigidos a la educación de los pacientes y sus familias, a la creación de planes de prevención y al desarrollo de programas de bienestar.

Los diferentes estudios FE y de CVRS realizados por la industria farmacéutica seguirán aumentando debido a que ésta tendrá que demostrar el valor de sus productos. El conocimiento de la terminología nos permitirá valorar e interpretar estos estudios.

Los profesionales sanitarios reforzaremos nuestra formación y habilidades para analizar los resultados de nuestros servicios, al conocer las preferencias y bienestar de los pacientes, y podremos utilizar esta información en la toma de decisiones terapéuticas, aumentando por tanto nuestra eficiencia.27

\section{Conclusión}

Nuestras acciones en el proceso del cuidado sanitario de los pacientes no solamente tienen resultados clínicos sino también económicos y humanísticos. El análisis de los mismos nos permitirá aumentar la calidad y eficiencia de los servicios que ofrecemos.

El número de estudios que valoran los resultados sanitarios sigue aumentando. Para aplicar e interpretar sus resultados correctamente es indispensable conocer la metodología y utilizar la terminología adecuadamente.

En un próximo futuro los pacientes asumirán un mayor grado de responsabilidad en la toma de decisiones, al tenerse en cuenta tanto las preferencias de los mismos como su bienestar y satisfacción con el cuidado sanitario. 


\begin{tabular}{|c|c|c|c|}
\hline \multicolumn{2}{|c|}{$\begin{array}{l}\text { Agradecimientos } \\
\text { Quisiera agradecer a los Dres. P. Monedero, J. Ca- } \\
\text { rrera'(Departamento de Anestesia), A. De la Peña (De- } \\
\text { partamento de Medicina Interna) de la Clínica Univer- }\end{array}$} & \multicolumn{2}{|c|}{$\begin{array}{l}\text { sitaria de Navarra, y al Dr. W. A. Gouveia (Director del } \\
\text { Departamento de Farmacia) del New England Medical } \\
\text { Center, Boston; sus comentarios para la confección fi- } \\
\text { nal de este trabajo. }\end{array}$} \\
\hline 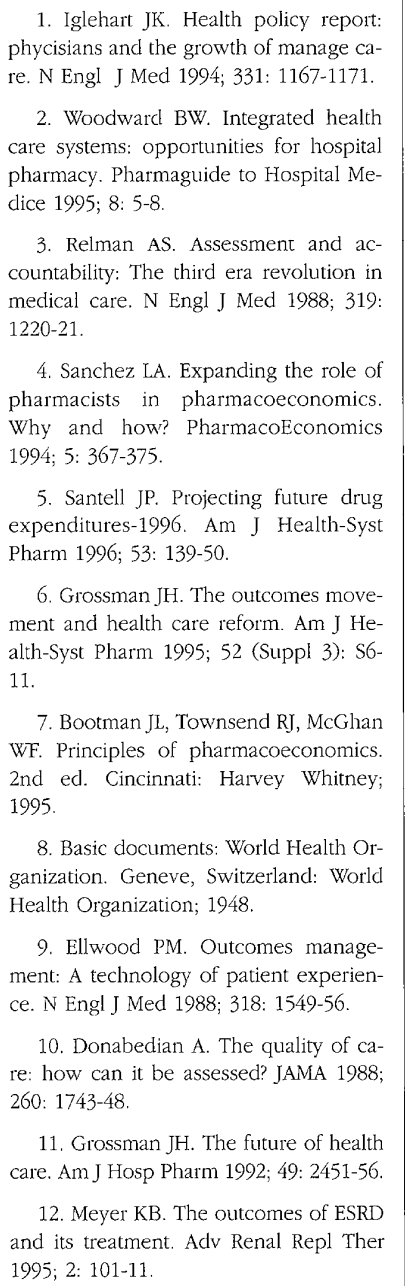 & \multicolumn{2}{|c|}{$\begin{array}{l}\text { 13. Kozma CM, Reeder CE, Schulz } \\
\text { RM. Economic, clinical, and humanistic } \\
\text { outcomes: a planning model for pharma- } \\
\text { coeconomic research. Clin Ther 1993; } 15 \text { : } \\
\text { 1121-32. } \\
\text { 14. Noncompliance with medications: } \\
\text { An economic tragedy with important im- } \\
\text { plications for health care reform. Balti- } \\
\text { more, Md: The Task Force for Complian- } \\
\text { ce; 1993. } \\
\text { 15. Johnson JA, Bootman JL. Drug-re- } \\
\text { lated morbidity and mortality. A cost-of- } \\
\text { illness model. Arch Intern Med 1995; } \\
\text { 155: 1949-56. } \\
\text { 16. Gagnon JP. A primer on Pharma- } \\
\text { coeconomics. Manage Care Med 1995; } 9 \\
\text { (2): } 11-18 . \\
\text { 17. Sacristan JA, Badia X, Rovira J, } \\
\text { eds. Farmacoeconomía: evaluación eco- } \\
\text { nómica de medicamentos. Editores mé- } \\
\text { dicos, Madrid, 1995. } \\
\text { 18. Drummond MF, Stoddart GL, To- } \\
\text { rrance GW. Basic types of economic eva- } \\
\text { luation. In: Methods for the economic eva- } \\
\text { luation of health care programs. Oxford: } \\
\text { Oxford Medical Publications, 1987; 7-8. } \\
\text { 19. Sanchez LA. Pharmaeconomic } \\
\text { principles and methods: including phar- } \\
\text { macoeconomics into hospital pharmacy } \\
\text { practice. Hosp Pharm 1995; } 29: 1035-40 \text {. } \\
\text { 20. Mehrez A, Gafni A. Quality-adjus- } \\
\text { ted life years, utility theory, and healthy- } \\
\text { years equivalents. Med Decis Making } \\
\text { 1989; 9: } 142-9 \text {. } \\
\text { 21. Udvarhelyi S, Colditz GA, Rai A, } \\
\text { Epstein AM. Cost effectiveness and cost } \\
\text { benefit analyses in the medical literature. } \\
\text { Ann Intern Med 1992; } 116: 238-244 . \\
\text { 22. Lee JT, Sanchez LA. Interpretation } \\
\text { of cost-effective and soundness of econo- } \\
\text { mic evaluations in the pharmacy literatu- } \\
\text { re. Am J Hosp Pharm 1991; } 48: 2622-27 .\end{array}$} & $\begin{array}{l}\text { 23. Sacristan JA, Soto J, Galande I. } \\
\text { Evaluation of pharmacoeconomics stu- } \\
\text { dies: utilization of a check-list. Ann Phar- } \\
\text { macother 1993; 27: 1126-23. } \\
\text { 24. Eisenberg JM. Clinical economics. } \\
\text { A guide to economic analyses of clinical } \\
\text { practices. JAMA 1989; 262: 2879-86. } \\
\text { 25. Task Furce on principles for eco- } \\
\text { nomic analysis of health care techno- } \\
\text { logy. Economic analysis of health care } \\
\text { technology. A report on principles. Ann } \\
\text { Intern Med 1995; 122: 61-70. } \\
\text { 26. Gouveia WA, Bungay KM. Incor- } \\
\text { porating Pharmacoeconomic principles } \\
\text { into hospital pharmacy practice. Top } \\
\text { Hosp Pharm Manage 1994; 13: } 31-37 \text {. } \\
\text { 27. Carrera-Hueso FJ, Giráldez J. Es- } \\
\text { tructuras de soporte en la toma de deci- } \\
\text { siones farmacoterapéuticas: El farmacéu- } \\
\text { tico. Farm Hosp 1995; 19(3): } 169-173 \text {. } \\
\text { 28. Patrick DL, Erickson P. Health sta- } \\
\text { tus and health policy. Allocating resour- } \\
\text { ces to health care. New York: Oxford } \\
\text { University Press, 1993. } \\
\text { 29 Anderson JP, Bush JW, Berry CC. } \\
\text { Classifying function for health outcome } \\
\text { and quality of life evaluation. Med Care } \\
\text { 1986; } 24 \text { : 454-69. } \\
\text { 30. Bergner M, Bobbit RA, Pollard } \\
\text { WE, Martin DP, Gilson BS. The Sick- } \\
\text { ness Impact Profile: Validation of a he- } \\
\text { alth status measure. Med Care 1976; 14: } \\
\text { 57-67. }\end{array}$ \\
\hline
\end{tabular}


33. Hunt SM, McEwen J, McKenna SP. Measuring health status a new tool for clinicians and epidemiologists. J R Coll Prac 1985: 35: $185-188$

34. Alonso J, Anto JM, Moreno C. Spa nish version of the Nothingham Health Profile: Translation and preliminary validity Am J Public Health 1990; 80: 704-708.

35. Wood-Dauphinee $S$, Williams JI. Reintegration to normal living as a proxy to quality of life. J Chronic Dis 1987; 40: 491-

36. Nelson EC, Landgraf JM, Hays RD, Wassan JH, Kirk JW. The COOP function charts: a system to measure patient function in physicians' offices. In: Lipkin M Jr, ed Functional Status Measurement in Primare Care. New York, NY: Springer-Verlag, 1990.

37. EuroQol( Group. EuroQol(-A new facility for the measurement of health related quality of life. Health Policy 1990; 16 199-208.
38. Parkerson GR, Broadhead WE, Tse CK. Comparison of the Duke Health Profile and the MOS Short-Form in healthy young adults. Med Care 1991; 29: 679-83.

39. Ware JE Jr, Sherbourne CD. The MOS 36-Item Short-Form Health Survey (SF-36). Med Care 1992; 32: 473-482.

40. Alonso J, Prieto L, Anto JM. La versión española del "SF-36 Health Survey" Cuestionario de Salud SF-36): un instrumento para la medida de los resultados clínicos. Med Clin (Barc) 1995; 104: 771-776.

41. Meenan RF. The AIMS approach o health status measurement: concepual background and measurement properties. J Rheumatol 1982; 9: 785-88.

42. Schipper H, Clinch A, McMurray A, Levitt M. Measuring the quality of life of cancer patients: the functional living index-cancer: development and validation. J Clin Onc 1984; 2: 472-483.
43. Testa MA, Anderson RB, Nackley IF, Hollenberg NK. Quality of life and antihipertensive therapy in men: a comparison of captopril with enalapril. N Engl J Med 1993; 328: 907-13.

44. Aaronson NK. Methodologic issues in assessing the quality of life of cancer patients. Cancer 1991; 67: $844-50$

45. Spilker B, ed. Quality of Life and Pharmacoeconomics in Clinical Trials, 2nd Ed. Philadelphia, PA: Lippincott-Raven, 1996.

46. Wagner AK, Vickrey BG. The rou tine use of health-related quality of life measures in the care of patients with epilepsy: rationale and reseach agenda Qual Life Res 1995; 4: 169-77.

47. Gouveia WA, Woodward BW Overview of managed care and integrated health care systems. CE Program, Elli Lilly and Company. 1995: 1-11. 


\section{Capítulo 2}

Carrera-Hueso FJ. Aplicación de los estudios farmacoeconómicos en el hospital. Med Clín (Barc) 1998; 111: 347-53. 


\section{REVISIONES}

\section{Aplicación de los estudios farmacoeconómicos en el hospital}

Francisco Javier Carrera-Hueso

Especialista en Farmacia Hospitalaria. Departamento de Farmacia y Tecnología Farmacéutica. Facultad de Farmacia Universidad de Navarra.

El costo de los medicamentos ha ido creciendo progresivamente en la última década dos o tres veces por encima de la inflación ${ }^{1}$ y las previsiones estiman que los nuevos medicamentos y tecnologías incrementarán el gasto un $2,8 \%{ }^{2}$ Mientras el aumento del costo de los nuevos medicamentos respecto a sus antecesores aumentaba continuamente, no quedaba claro que éstos también mejorasen la esperanza la calidad de vida de los pacientes ${ }^{3}$.

A pesar de que los medicamentos constituyen sólo una pequeña parte del gasto sanitario, éstos todavía merecen la atención de las Administraciones sanitarias por al menos estas cuatro razones ${ }^{4}$ : el gasto sanitario continúa creciendo, los medicamentos son considerados como productos más que como servicios, algunos productos farmacéuticos aportan un dudoso beneficio y existe una conciencia generalizada de que los nuevos productos biotecnológicos aumentarán el gasto farmacéutico hasta niveles sin precedentes. Los métodos de contención del gasto irán dirigidos al disminuir melodos de con hospitalarias, acotar los dias de las admisiones hospitalanias, acotar los dias de utilizar menos tratamientos intensivos (cuidados intensivos, intervenciones quirúrgicas, etc.) y propiciar el aumento y la utilización de los servicios ambulatorios (médicos y quirúrgicos)

Tradicionalmente, la medicina estaba más preocupada por sanar o paliar el efecto de las enfermedades que en mejorar el bienestar social. Para ello utilizaba tres indicadores para evaluar sus resultados: la mortalidad, la morbilidad y el cos$\mathrm{te}^{3}$. Kozma et al ${ }^{5}$ diseñaron un modelo gráfico para comprender el complicado proceso de la toma de decisiones farmacoterapéuticas en el que no sólo se miden los resultados clínicos (derivados de una enfermedad o tratamiento morbilidad y mortalidad) en el tratamiento de los pacientes, sino también los resultados económicos (comparan los costes y las consecuencias de las distintas alternativas terapéuticas) y humanísticos (consecuencias de la enfermedad sobre $y$ huma funcionat del paciente o medidas sol bre el estado funciona del paciente o medidas sobre . El mode lo también contempla la existencia de una serie de intermediarios o modificadores del tratamiento que pueden afectar a los resultados, como el grado de cumplimiento de los tratamientos y las reacciones adversas de los medicamentos. Para realizar valoraciones de la calidad del cuidado sanitario, Donabedian ${ }^{6}$ estableció que debían definirse los componentes del mismo y describió la relación existente entr tario (fig. 1)

Correspondencia: Dr. F.j. Carrera-Hueso.
Caudillo, 127.46135 Albalat dels Sorells. Valenci

Manuscrito aceptado el 1-10-1996

Med Clin (Barc) 1998; 111: 347-353
El interés por los estudios farmacoeconómicos (FE) ha ido aumentando progresivamente en la última década de tal manera que la media de estudios por empresa farmacéutica considerando variables económicas ascendió del 1,7\% en 1988 al $23,7 \%$ en $1994^{7}$. Esta tendencia parece que se mantendrá en el futuro, debido a que los laboratorios farmacéuticos necesitan demostrar en un mercado altamente competitivo el valor de sus productos.

Aunque todavía existen algunos problemas de estandarización sobre los aspectos metodológicos utilizados en los estudios FE, actualmente hay suficientes directrices para considerar a los estudios FE como una herramienta útil para la toma de decisiones farmacoterapéuticas ${ }^{4.8-15}$. En 1994, un grupo de expertos realizó una propuesta de estandarización de algunos aspectos metodológicos de los análisis costeefectividad y coste-utilidad en la evaluación de tecnologias y programas sanitarios a la Subdirección General de Prestaciones y Evaluación de Tecnologís Sanitarias ${ }^{16}$. Recientemente el Colegio de Médicos Americanos ha difundido unas recomendaciones para la correcta realización y valoración de estos estudios ${ }^{17}$.

Grossman ${ }^{3}$ argumenta que las razones para valorar los resultados sanitarios son: definir, evaluar y mejorar la efectividad del servicio prestado, controlar los costes de los servicios superffuos o inapropiados y documentar el valor del cuidado sanitario.

Los objetivos del presente trabajo son: describir los distintos estudios FE, identificar sus potenciales aplicaciones y enumerar las estrategias que pueden realizar los departamentos y servicios hospitalarios para su incorporación en la toma de decisiones.

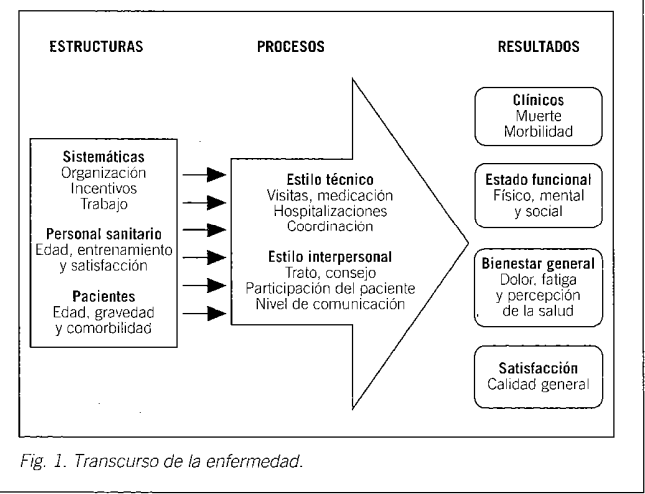


Resultados y discusión

MEDICINA CLIINICA. VOL. 111. NÚM. 9. 1998

\section{Tipo de estudios farmacoeconómicos}

La farmacoeconomía es la descripción y el análisis de los costes del tratamiento con fármacos en los sistemas de sa lud y en la sociedad 18 También puede definirse como la determinación de la eficiencia (relación entre costes y efectos) de un tratamiento farmacológico y su comparación con otras opciones, con el fin de seleccionar aquella con una relación coste-beneficio más favorable ${ }^{9}$. En un sentido más práctico se puede definir como la ciencia que sitúa el valor de la farmacoterapia ${ }^{19}$. La farmacoeconomía se engloba dentro de la evaluación económica de tecnologías sanitarias.

Los estudios FE identifican, miden y comparan los costes (p. ej., recursos consumidos) y las consecuencias (clínicas, económicas y humanísticas) de los productos farmacéuticos y servicios. Estos estudios incluyen, además de las evaluaciones descritas en la tabla 1, las evaluaciones humanísti cas, los estudios de coste de una enfermedad y los análisi de decisión. Las preferencias y satisfacción de los pacientes y los estudios de calidad de vida relacionada con la salud (CVRS) se engloban dentro de las evaluaciones humanisticas4. En la tabla 1 se describen las caracteristicas de las principas (a) evaluaciones economicas 8 . Los tipos de análisis se diferencian entre sí por cómo miden los efectos de los tratamientos, ya que todos miden los costes en unidades monetarias. Los costes son la magnitud de los recursos consumidos y pueden ser directos, indirectos e intangibles. Los costes directos pueden ser sanitarios: los relacionados con los medicamentos y el cuicado médico ( $p$. ej. coste de dispensación y administración del medicamento, hospitalizaciones, ción y administración del medicamento, hospitalizaciones, consultas, pruebas relacionados con el suministro de servicios médicos ( $p$. ej. transporte del paciente, cuidado familiar, comida especia etc.). Los costes indirectos son los debidos a la reducción de la capacidad productiva de los pacientes debido tanto a la morbilidad ( $p$. ej., dias de absentismo y sueldos no cobrados) como a la mortalidad ( $p$. ej., pérdidas por muerte prematura) ${ }^{10}$ Los costes intangibles son los debidos al dolor y maturamiento dos costes intangibles son los debidos al dolor y dad o del tratamiento, y éstos no suelen incluirse en los análisis debido a la extremada dificultad de poder cuantificarlos. Los costes pueden ser variables o fijos, dependiend si la cantidad de resultado obtenido varía o no. Según la du ración del estudio, más de un año normalmente, los costes y también los beneficios deben actualizarse. La tasa de descuento refleja la preferencia temporal y el rendimiento de las inversiones en una sociedad, por lo que es utilizada las inversiones en una soc la actualización del dinero. Se suele utilizar una tasa de descuento para los costes que oscila entre el 2 y el $6 \% 20$

TABLA 1

Tipo de estudios farmacoeconómicos

\begin{tabular}{|c|c|c|c|}
\hline Tipo de análisis & Medida de los costes & Medida de los resultados & Interpretación del análisis* \\
\hline Minimización de costes (EMC) & Unidades monetarias & $\begin{array}{l}\text { No se miden } \\
\text { Se asume que son equivalentes }\end{array}$ & $\mathrm{C}_{1}<\mathrm{C}_{2}=$ aceptar 1 \\
\hline Análisis coste-beneficio (ACB) & Unidades monetarias & Unidades monetarias & $\begin{array}{l}\mathrm{B} / \mathrm{C}<1=>\text { aceptar } 1 \\
\mathrm{~B} / \mathrm{C}=1=>\text { iguales } \\
\mathrm{B} / \mathrm{C}<1=>\text { rechazar } 1\end{array}$ \\
\hline Análisis coste-efectividad (ACE) & Unidades monetarias & $\begin{array}{l}\text { Unidades clínicas habituales, físicas on naturales } \\
\text { (p. ej,. años de vida ganados, mmHg de TA reducidos } \\
\text { o estancias hospitalarias ahorradas) }\end{array}$ & $\begin{array}{l}\mathrm{C} / \mathrm{E}=+/-=>\text { rechazar } \\
\mathrm{C} / \mathrm{E}=-1+=>\text { aceptar } \\
\mathrm{C} / \mathrm{E}=-1-=>\text { ? } \\
\mathrm{C} / \mathrm{E}=+/+=>\text { ? }\end{array}$ \\
\hline Análisis coste-utilidad (ACU) & Unidades monetarias & $\begin{array}{l}\text { Unidades de cantidad y calidad de vida (p.ej., años de vida } \\
\text { ajustados por calidad, AVAC) }\end{array}$ & $\begin{array}{l}C / U=+l=>\text { rechazar } \\
C / U=-1+=>\text { aceptar } \\
C / U=-1->? \\
C / U=+/+=>?\end{array}$ \\
\hline
\end{tabular}

Los estudios de minimización de costes no miden las consecuencias porque suponen que los efectos de las alternativas son equivalentes basándose en publicaciones previas sobre eficacia. Estos estudios son los más utilizados en la toma de decisiones de formularios o guías farmacoterapéuticas $^{4}$

Los estudios coste-beneficio permiten comparar distintos programas o tratamientos, pero tienen la dificultad de asignar valores monetarios a los resultados y no permiten comparar simultáneamente productos con más de una indicación ${ }^{7}$. Inicialmente fueron los más utilizados, pero su baja aceptación por los profesionales sanitarios es debida a que estos estudios asignan un valor monetario a la vida humana?

Los estudios coste-efectividad son los utilizados con más frecuencia debido a que se ajustan mejor a los resultados de la práctica clínica diaria 7,10 . Estos estudios no permiten la comparación entre medicamentos administrados en distintas indicaciones, pero son los más apropiados para medir los resultados en términos de obtener un específico objetivo terapéutico ${ }^{10}$

Los estudios coste-utilidad miden los efectos a través de una unidad que integra la cantidad y calidad de vida, años de vida ajustados por calidad (AVAC) , $, 8,9^{9}$. Otra unidad utilizada en estos estudios son los años de vida sanos equivalentes ${ }^{21}$. LOS AVAC se obtienen calculando los años de vida ganados ponderándolos con la calidad de vida por técnicas que miden los estados de salud basadas en preferencias o utilidades. Estas técnicas son: escalas de categorías, juego estándar, estimación de la magnitud, equivalencia tempora y equivarencia de personas 89 . Los estados de salud obtendos pueden agregarse obteniendo una puntuación global, a partir de la que se calculan los AVAC para los estudios coste-utilidad. LOS AVAC también pueden calcularse me diante la utilización de métodos indirectos usando las medidas de utilidad o escalas de salud, que se describirán posteriormente.

Jolicoeur et $\mathrm{a}^{22}$ enumeraron los distintos pasos a seguir para la realización de estos estudios en el ambiente hospitalario, sin necesidad de utilizar grandes recursos, para obtener resultados suficientemente fiables (tabla 2). Esta estrategia nos da una visión acerca de la metodología utilizada en las evaluaciones económicas.

Dos puntos importantes a tener en cuenta son el análisis de sensibilidad y el análisis incremental. El análisis de sensibilidad recoge el impacto que tienen las variaciones (normazadas en el resultado final del estudio, proporcionando una mayor solidez a los resultados. Las estimaciones que deben 
Resultados y discusión

F.J. CARRERA HUESO.- APLICACIÓN DE LOS ESTUDIOS FARMACOECONÓMICOS EN EL HOSPITAL

ser testadas por el análisis de sensibilidad son ${ }^{4}$ los grados de eficacia asumidos en cada intervención, el grado de reacciones adversas de cada medicamento, las estimaciones de los costes más importantes y la tasa de descuento elegida. Los resultados del estudio deben presentarse utilizando el análisis incremental, que es el coste adicional cuando se compara una alternativa con la siguiente y no debe confundirse con el análisis marginal (efectividad por unidad adicional de coste)

La CVRS es el valor asignado a la duración de la vida modificado por la disminución del estado funcional, percepciones y oportunidades sociales debido a una enfermedad, accidente, tratamiento o política ${ }^{23}$. En los estudios de CVRS, el paciente es la principal fuente de información para evaluar los tratamientos farmacológicos o los programas sanitarios y estos estudios proporcionan una nueva información a los clínicos ${ }^{12}$. Para medir la CVRS se utilizan cuestionarios que pueden ser genéricos o específicos. Los cuestionarios son administrados por entrevistadores (personalmente o por teléfono) o autoadministrados (en las clínicas, supervisado por el entrevistador o no y por correo)

Los cuestionarios genéricos evalúan todos los aspectos relevantes de la salud y no están influenciados por el tipo de enfermedad, raza edades, etc. Son multidimensionales, es decir, son aplicables tanto a la población en general como a los pacientes con cualquier tipo de enfermedad, y su principal limitación es intercultural. Los cuestionarios genéricos evalúan al menos cuatro conceptos del estado de la salud: la función física, la función mental, la función de relaciones sociales y del trabajo, y las percepciones generales de la salud (tabla 3$)^{24}$ Los más ampliamente utilizados son. Nottingham Health Profile, Sickness Impact Profile y e! SF-36 Health Survey, que han sido adaptados para su utilización en la población española $25-27$

Las medidas de utilidad o escalas también evalúan los principales estados de salud y los niveles de salud obtenidos pueden, en algunos cuestionarios, agregarse en una única puntuación obteniendo el índice de salud, a partir del que se calculan los AVAC. Los instrumentos más utilizados para el cálculo de los AVAC son: Escala del bienestar ${ }^{28}$. EuroQol ${ }^{\$ 29}$. Health Utilities Index y el Index of Health-related Qol ${ }^{\circledR 29}$, Health

quality of life ${ }^{4}$.
Los instrumentos específicos asumen que cada condición Los instrumentos especificos asumen que cada condición las funciones afectadas por esa condición ${ }^{24}$. Estos cuestionarios valoran sólo aquellos aspectos importantes de una enfermedad y su principal inconveniente es que sólo pueden aplicarse en los casos para los que fueron diseñados y no permiten comparaciones entre enfermedades; sin embargo, tienen la ventaja de presentar una alta sensibilidad a los cambios clínicos.

Para medir la CVRS en los pacientes con tratamientos far macológicos, Aaronson ${ }^{30}$ propuso la utilización de un test genérico y otro específico, de esta manera obtendríamos, por una parte información más defallada y sensible a los cambios clínicos y, por otra, podríamos comparar los resultados con otras enfermedades.

Los instrumentos deben cumplir una serie de requisitos antes de ser utilizados como herramientas para medir y valora la CVRS de los pacientes. Éstos son ${ }^{31}$; fáciles de usar, interpretar y administrar, aplicables a diferentes poblaciones; se fiables, válidos y prácticos; deben incluir aspectos del bienestar de los pacientes, tener un amplio rango de medidas, y ser comprensivos.

A diferencia de los estudios FE los distintos grupos de investigadores han ido desarrollando sus propios cuestionarios, lo que impide la comparación entre estudios publica-
TABLA 2

Pasos a seguir para la realización de estudios farmacoeconómicos

\section{Definir el problema $\mathrm{FE}$}

2. Determinar la perspectiva del estudio

Determinar las distintas alternativas y los resultados obtenidos

con las mismas

4. Seleccionar el tipo de análisis más apropiad

5. Dar valor económico a los resultados de las distintas alternativ

. Identificar los recursos disponibles para realizar el estudio

darse de cada resultado

9. Actualizar los costes y/o realizar un análisis de sensibilidad

10. Presentar los resultados a los profesionales más adecuados

FE: farmacoeconómico.

TABLA 3

Conceptos generales de salud ${ }^{24}$

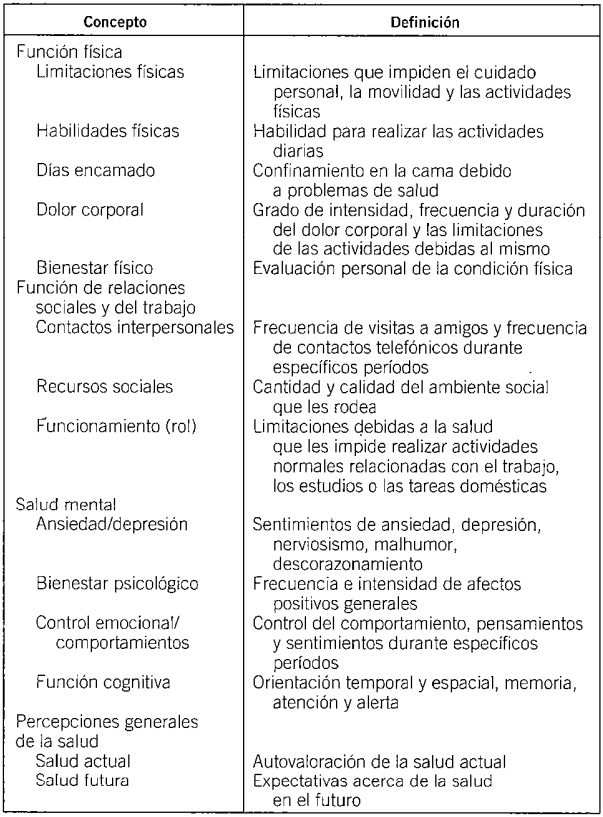

dos $^{31}$. Hay un número de cuestiones metodológicas que deben considerarse en la valoración de estudios de CVRS, como qué instrumento debe utilizarse (general o específico), qué es más útil medir el índex o los perfiles, qué dimensiones deben medirse y cuáles no, la relativa importancia de las dimensiones medidas en el estado global de CVRS, la fiabilidad y la validez de los instrumentos.

Pese a las limitaciones metodológicas existentes en la actualidad, los estudios FE son una herramienta útil para orientar la toma de decisiones farmacoterapéuticas.

\section{Aplicación de los estudios farmacoeconómicos}

Las potenciales aplicaciones de la farmacoeconomía en los sistemas sanitarios son s, $, 9,19^{2}$ : autorización para la comercia- 
Resultados y discusión

MEDICINA CLÍNICA. VOL 111. NÚM 9. 1998

lización de medicamentos, fijación de precios, financiación pública de medicamentos, soporte de las decisiones sobre investigación y desarrollo en la industria farmacéutica, definir estrategias de márketing en la industria farmacéutica, su utilización en la investigación clínica con medicamentos, incorporación de medicamentos a formularios o guías farmacoterapéuticas, y como soporte en la toma de decisiones farmacoterapéuticas.

Autorización para la comercialización de medicamentos

Las autorizaciones sanitarias de Australia ${ }^{32}$ y Ontario (Canadá) ${ }^{33}$ han dictado unas normativas en las que se deben incluir resultados FE en el proceso previo a la aprobación de un medicamento. Estas dos normativas son muy semejantes y se diferencian principalmente en que en la normativa australiana no es necesario recoger los costes indirectos para la realización de los estudios FE.

Aunque estas normativas han sentado un precedente, parece improbable que de momento estos análisis tengan un importante papel en el proceso de aprobación de un nuevo medicamento, que seguirán basándose en criterios de seguridad, eficacia y calidad ${ }^{4}$

Fijación de precios

La incorporación de los estudios FE puede situar el valor teapéutico del medicamento justificando el precio del mismo $^{7,19}$. En nuestro país existe una normativa que contempla la utilidad terapéutica del medicamento ${ }^{34}$. Esta estrategia puede ser utilizada en la negociación de precios con las autoridades sanitarias, de forma que un medicamento pueda tener un mayor o menor precio de venta dependiendo de sus ventajas terapéuticas respecto a otras alternativas. La incorporación de resultados FE en la fijación de precios puede potencialmente crear otro punto de controversia entre reguladores y la industria farmacéutica ${ }^{4}$

También los estudios FE pueden utilizarse en la negociación de precios d nitiva, el objetivo es dar valor al medicamento y justificar su precio

Financiación pública de medicamentos

En nuestro sistema sanitario de cobertura universai, existen dislinlias listas de medicarmentus que puederr ser firranciados (parcialmente o en su totalidad) o no por el mismo, dependiendo de la aportación terapéutica del sistema y del grupo de pacientes a los que se les aplica diferente cobertura (p. ej., jubilados y diabéticos $)^{9}$

Las exclusiones pueden ser generalizadas (por grupos terapéuticos) o indivicualizadas y pueden aplicarse tanto a los nuevos medicamentos como a los comercializados con anterioridad ${ }^{35}$. Los estudios FE de medicamentos pueden asistir en la decisión del grado de financiación de un medicamento en alguno de los grupos anteriormente citados, teniendo o no en cuenta el precio de los mismos.

Soporte de las decisiones sobre investigación y desarrollo en la industria farmacéutica

La gestión empresarial y los economistas han aplicado el análisis de decisión desde la Segunda Guerra Mundial como base de la toma de decisiones. De la misma manera, en la industria farmacéutica las decisiones están basadas en un análisis de decisión?

La incorporación de los estudios FE a los análisis de decisión puede servir para identificar, analizar y decidir las áreas más prometedoras para la investigación y desarrollo de nuevos medicamentos.

Definir estrategias de márketing en la industria farmacéutica El objetivo de los estudios de vigilancias de mercados es conocer la experiencia de la utilización de los medicamentos para poder revaluar en términos económicos su eficiencia ${ }^{9}$.

La utilización de los estudios FE en este marco de actuación puede ayudar en la modificación de precios, incorporación en formularios, recomendaciones terapéuticas, e incluso cambios en la presentación de formas farmacéuticas y/o dosificación del medicamento.

Utilización en la investigación clínica con medicamentos

La incorporación de los estudios FE en los ensayos clínicos tiene el inconveniente de que se obtienen resultados de eficacia, lo que no puede coincidir con la utilización del medicamento en la práctica clínica (efectividad). Normalmente se inician estos estudios en ensayos clínicos en fase III y se prolongan a estudios poscomercialización o en fase IV. La aplicación de los estudios FE en ensayos clínicos es ampliamente revisada en otras referencias 4.9

\section{Incorporación de medicamentos a formularios} o guías farmacoterapéuticas

La Comisión de Farmacia y Terapéutica (CFT) debe establecer y mantener la seguridad y el uso apropiado de los medicamentos con una relación coste-efectividad razonable, y su camentos con una relación coste efectividad razonable, y su mayor responsabilidad es la elaboración y mantenimiento actualizado del formulario o guía de medicamentos. En otras palabras, la CFT debe promover un uso racional del medicamento en su hospital o área de actuación.

La gestión efectiva de un formulario es aquella que selecciona los medicamentos por su seguridad y eficacia minimizando redundancias terapéuticas y maximizando la relafármacos del mismo que no presenten una ventaja cualitativa ${ }^{15}$

La CFT debe priorizar sus esfuerzos en la protocolización de los siguientes medicamentos ${ }^{13}$ : los que sean más iatrogénicos (p. ej., aminoglucósidos); los utilizados en pacientes de alto riesgo ( $\mathrm{p}$. e.j., pediátricos); los que representan un mayor coste económico, ya sea porque se utilizan en un mayor número de pacientes ( $\mathrm{p}$. ei., antihistamínicos $\mathrm{H}_{2}$ ) o por su (los que su empleo represente un riesgo añadido para los pacientes (p. ej., medicamentos en ensayo clínico y citostáticos), y los que impliquen al mayor número de departamentos médicos (p. ej., albúmina).

Para lograr este objetivo la CFT se vale de diferentes estrategias $^{14}$ : a) añadir, restringir o eliminar medicamentos del forsarrolladas por la industria farmacéutica; c) evaluar los resultados de la utilización de medicamentos (p. ej., política de antibióticos), y d) definir criterios de uso de ciertos medicamentos (p. ej., albúmina)

La incorporación de datos económicos en la gestión del formulario sirve de soporte para que las decisiones sean más eficientes. En la gestión de los formularios han empezado a cientes y la calidad de vida ${ }^{15}$, pero normalmente no se dispone de esta información ${ }^{12}$

Los costes indirectos raramente son utilizados en este tipo de decisiones y el análisis más usual para soportar las deci- 
Resultados y discusión

F.J. CARRERA HUESO.- APLICACIÓN DE LOS ESTUDIOS FARMACOECONÓMICOS EN EL HOSPITAL

siones en la gestión de un formulario es el de minimización de costes. La utilización del análisis de decisión es un factor primordial en la toma de decisiones ${ }^{4}$.

Soporte en la toma de decisiones farmacoterapéuticas

La aplicación clínica de los estudios FE puede beneficiar tanto a los pacientes como al propio equipo asistencial, y la sociedad en su conjunto por incrementar la calidad de la asistencia prestada al paciente y racionalizar recursos. Las decisiones pueden ser ${ }^{13,14}$ : a) sobre la incorporación o no del medicamento al formulario, b) sobre el tratamiento farmacoterapét extrapolar los resultados al tratamiento de un paciente concreto, ya que éste puede no tener las mismas características poblacionales que la muestra del estudio $\mathrm{FE}^{12}$; c) sobre las normas de utilización de un medicamento. Por ejemplo, qué régimen de dosificación de un antiemético es el más coste-efectivo para nuestro hospital, y d) justificación de programas o servicios de un departamento. Por ejemplo, en la implantación de la distribución de medicamentos en dosis unitarias o de un servicio de radiología intervencionista en el hospital. El conocimiento y la valoración de las técnicas de los estudios FE pueden servirnos como herramientas para valorar los resultados obtenidos de los servicios que ofrecemos a los pacientes.

\section{Estrategias para la incorporación de los estudios}

\section{farmacoeconómicos en el hospital}

El número de estudios clínicos publicados que incorporan variables económicas de la salud ha aumentado exponencialmente, y el porcentaje de estos estudios respecto al total fue, para los años 1988 y 1994 , del 2,6 y del $28 \%$, respectivamente?

Lerspectiva que tomaremos cuando realicemos un estudio FE será departamental o del hospital. Otras perspectivas como aseguradoras, Administración y la sociedad nos serán útiles al analizar los trabajos publicados, ya que sus resultados y conclusiones son más fácilmente extrapolables.

La utilización apropiada de la terminología y el conocimiento de la metodología son indispensables para aplicar e interpretar correctamente los resultados de los estudios FE.

Los posibles beneficios que pueda aportar la incorporación de los estudios FE, tanto a los departamentos y servicios como a los equipos multidisciplinarios de atención al paciente, son: elegir entre alternativas terapéuticas para una indicación (decisión de formulario), poder justificar los programas o servicios implantados por el departamento, como soporte en la toma de decisiones farmacoterapéuticas, y puede proporcionar información valiosa para aplicarla en los estudios de utilización de medicamentos en el hospital.

Tres estrategias pueden ayudar a implantar la farmacoeconomía en los servicios y departamentos hospitalarios, y éstas son 14. utilizar la bibliografía publicada construir un modelo económico y realizar un estudio FE específico en nuestro hospital.

\section{Utilizar la bibliografía publicada}

La revisión de la bibliografía de estudios FE puede servirnos de soporte en la toma de decisiones farmacoterapéuticas ${ }^{12}$ Diversos estudios demuestran que tanto en la bibliografía médica ${ }^{36}$ como en la farmacéutica ${ }^{37}$ existen interpretaciones erróneas de la terminología, así como de la metodología utilizada en los estudios FE. Todos los estudios utilizaron los mismos métodos para analizar los artículos publicados.
Lee y Sánche $z^{37}$ analizaron 75 estudios publicados en revistas farmacéuticas entre enero de 1985 y diciembre de 1990, y obtuvieron los siguientes resultados: los estudios fueron deficientes en 7 de los 10 criterios analizados $^{8}$, solamente el $31 \%$ de los estudios identificaron todos los costes y consecuencias relevantes para cada alternativa, tan sólo un estudio realizó el análisis incremental de los resultados y el análisis de sensibilidad fue realizado en tan sólo el $6 \%$ de los trabajos. Más de la mitad de los estudios publicados utilizó inapropiadamente el término coste-efectividad, interpretando el mismo como costes ahorrados. Estos resultados nos indican que debemos de ser bastante críticos al valorar este tipo de estudios.

Afortunadamente, disponemos de guías o chek-list para realizar una valoración en profundidad de los trabajos publicados. En la tabla 4 se recogen los criterios para la evaluación de estos estudios 8,10,38,39.

de estos estudios, mos valorar si los resultados y conclusiones del mismo son o no extrapolables a nuestro medio. Para ello, deberemos

TABLA 4

Criterios para evaluar publicaciones de estudios farmacoeconómicos ${ }^{14,19,38}$

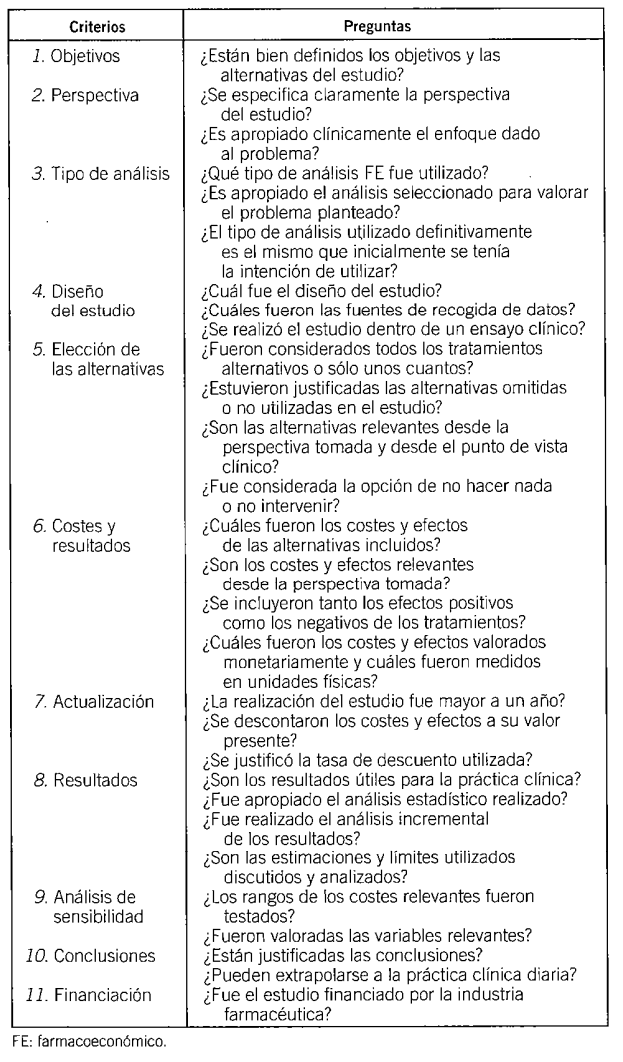


Resultados y discusión

MEDICINA CLINICA. VOL. 111. NÚM. 9. 1998

trasladar los resultados de eficacia obtenidos en un ensayo clínico a resultados de efectividad, comparar la población del estudio con nuestra población susceptible de recibir e tratamiento, y trasladar tanto los costes relevantes del estudio a la perspectiva con la que vamos a tomar nuestra decisión como los resultados relevantes del estudio a los resultados clínicamente más importantes ${ }^{4,10,14}$

dos clínicamente más importantes ${ }^{4.10,14}$ Podemos utilizar el análisis de sensibilidad presentado en los estudios FE publicados aplicando las estimaciones regis tradas en nuestro ámbito (p. ej., número de infecciones nosocomiales en nuestro hospital). También se puede diseñar un árbol de decisión, según nuestras necesidades, con una combinación de datos del estudio y de nuestro entorno tanto para los costes como para las consecuencias ( $P$. ej. dias de estancia) $)^{14}$

Las ventajas de utilizar los estudios FE publicados son: se realiza un análisis midiendo la calidad de los estudios, los datos son rápidamente obtenidos para su uso inmediato se necesitan pocos recursos, y es barato. En cambio, sus principales desventajas son la dificultad para generalizar las conclusiones de los estudios y la variación de la calidad de los mismos.

Utilización de modelos farmacoeconómicos

Los modelos FE utilizan distintas fuentes de información clinicas y epidemiológicas para obtener suficientes datos que permitan comparar las alternativas terapéuticas. Fstas fuentes pueden ser: resultados de ensayos clínicos, estudios observacionales, opiniones de expertos, estudios epidemiológi$\cos$, registros hospitalarios y cualquier otra base de datos disponible 4,9

La mayoría de los modelos se basan en técnicas de análisis de decisión, cuya principal herramienta es el árbol de decisión 8,40 . Los análisis de decisión predicen, identifican y valoran o cuantifican la posibilidad de que se produzcan las consecuencias de cada alternativa ${ }^{40}$.

El árbol de decisión permite reducir a un único valor todas las variables consideradas permitiendo la comparación de los tratamientos o alternativas ${ }^{40}$. A menudo no disponemos de todos los datos necesarios para construir el árbol, por lo que realizaremos suposiciones para completarlo. Estas suposiciones son el talón de Aquiles de los modelos FE, y por eso algunos autores consideran que estos modelos tienen poco rigor científico. Se puede mejorar la calidad de los modelos FE utilizando técnicas metaanalíticas para seleccionar los estudios publicados y aumentar el tamaño de la muestra estudiada? .

La ventaja de utilizar estos modelos es que reflejan mejor la práctica clínica usual o la efectividad que los estudios realizados protocolizados en los ensayos clínicos y no consumen excesivos recursos para llevarlos a cabo ${ }^{14,40}$.

Junto al número de suposiciones al diseñar el modelo FE, su principal desventaja es el grado de dificultad para diseñarlos y aplicarlos correctamente, y se necesita personal altamente cualificado y familiarizado con estas técnicas.

Realización de un estudio farmacoeconómico

Muchas veces no dispondremos de estudios publicados para apoyarnos en ellos antes de la toma de decisiones o los que existen no son trasladables a nuestro medio, y entos que existen no son trasladables a nuestro medio, y entonces necesitare

En la tabla 2 se indican los distintos pasos a seguir para la realización de estos estudios sin necesidad de utilizar grandes recursos ${ }^{22}$. Es aconsejable que los estudios sean realizados por equipos multidisciplinarios integrados por profe- sionales interesados en las posibles decisiones que se adoptarán como consecuencia de los resultados obtenidos ${ }^{23}$. Por ejemplo, la incorporación de un microbiólogo y/o un especialista en enfermedades infecciosas en la CFT permite, además de una mejor selección del antibiótico más costeefectivo para su incorporación al formulario, una mejor utilización del mismo en el futuro.

Una vez realizados todos los pasos anteriormente citados,

tos pasos anteriorm debemos considerar la posibilidad de hacer un seguimiento 0 auditoría de los resultados obtenidos ${ }^{14}$. Esto es, realizar estudios de utilización del medicamento y corroborar por ejemplo el grado de eficacia obtenido o los costes del tratamiento obtenidos en el estudio piloto.

Estos estudios, a pesar de las dificultades de aleatorización, obtienen datos fiables de efectividad y su diseño puede reanecidades de nuestro hospital, siendo esta sil principal ventaja.

L gran desventaja de realizar un estudio FE es su alto coste, tanto en tiempo como por la cantidad de recursos utilizados. Otro inconveniente es que no podremos tomar una decisión hasta que haya transcurrido el tiempo necesario para la obtención y análisis de los resultados. Obviamente, no será posible realizar estos estudios para cada decisión y deberemos priorizar para que nuestras decisiones sean más fectiva ${ }^{13}$. Centraremos nuestros esfuerzos en los siguientes efectivas centra para que nuestras decisiones seantes camentos de alto coste ya sea porque se puedan utilizar en un mayor número de pacientes o por su alto coste individual; c) medicamentos réplicas o me-too; d) medicamentos de dudosa eficacia, y e) nuevos medicamentos comercializados.

\section{Conclusión}

Los estudios FE identifican, miden y comparan los costes y las consecuencias (clínicas, económicas y humanísticas) de los productos farmacéuticos y servicios. La utilización apropiada de la terminología y el conocimiento de la metodología son indispensables para aplicar e interpretar correctamente los resultados de los estudios FE.

Las potenciales aplicaciones de los estudios FE en los servicios farmacéuticos son: la gestión de formularios o guías farmacoterapéuticas (incorporación de un nuevo medicamento, revisión de grupos terapéuticos y estudios de utilización de medicamentos), como soporte en la toma de decisiones farmacoterapéuticas y para justificar los servicios que ofrecen los departamentos hospitalarios al equipo multidisciplinario y al paciente.

Distintas cstratcgias pueden incorporar las técnicas farmacoeconómicas en el hospital y su integración en el proceso de toma de decisiones beneficiará tanto a los pacientes como al hospitaly a la sociedad en su coniunto por utilizar los recursos más eficientemente.

\section{REFERENCIAS BIBLIOGRÁFICAS}

1. Gouveia WA, Woodward BW. Overview of managed care and integrated 1. health care systems. CE Program, Elli Lilly and Company, 1995; 1: 1-11. 2. Santell JP. Projecting future drug expendind
Pharm 1996; 53: 139-150. Grossman $1 H$ The outcon

movement and health care reform. Am J Bootman JL, Townsend RJ (Supl 3): 6-11.

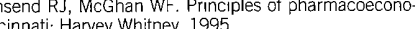

Koz CM, Reeder CE, Schulz RM. Economic, clinical, and humanistic outcomes: a planning model for pharmacoeconomic research. Clin The . Donabedian A. The quality of care: how can it be assessed? JAMA 1988 260: 1.743-1.748 
Resultados y discusión

F.J. CARRERA HUESO.- APLICACION DE LOS ESTUDIOS FARMACOECONÓMICOS EN EL HOSPITAL

7. Gagnon JP. A primer on pharmacoeconomics. Managed Care Med 1995: 2: 11-18

8. Drummond MF. Stoddart GL, Torrance GW, editores. Methods for the economic evaluation of health care programs. Oxford: Oxford Medica

9. Sacristan JA. Badia X. Rovira J, editores. Farmacoeconomia: evaluación Sánchez LA Pharicamentos. Madrid. Editores médicos, 1995

macoeconomics into hospital pharmacy practice. Hosp Pliars 1995: 29 1.035-1.040

11. Bakst A. Pharmacoeconomics and the formulary decision-making process. Hosp Formul 1995; 30: 42-48.

12. Gouveia WA, Bungay KM. incorporating pharmacoeconomic principles (1994; 13

13. Carrera-Hueso FJ, Giráldez J. Estructuras de soporte en la toma de deci173

14. Sánchez LA. Pharmacoeconomics and formulary decision making. Pharmacoeconomics 1996; 9 (Supl 1): 16-25.

15. Skaer Th. Applying pharmacoeconomics and quality-of-life measures to the formulary management process. Hosp Formul 1993; 28: 577-584. tarias. Propuesta de estandarización de algunos aspectos metodológicos de los análisis coste-efectividad y coste-utilidad en la evaluación de tecnologias y programas sanitarios. Madrid: Ministerio de Sanidad y Consu-

17. Task Force on principles for economic analysis of health care technology. Economic analysis of health care technology. A report on princi-
ples. Ann Intern Med 1995; 122: 61-70. Towsend JR. Postmarketing drug research and development. DICP 1987: 21: $134-136$

19. Sánchez $L A$. Expanding the role of pharmacists in pharmacoeconomics Why and how? Pharmacoeconomics 1994: 5: 367-375.

21. Mehrez A, Gefny. Phanacoeconod 1996; 9: 179-182. Mearez A. Gatni A. Quality-adjusted life years, utility theory, and healthy. Patrick DL, Erickson P. Health status and healthy policy. Allocating re sources to health care. Nueva York: Oxford University Press, 1993. Bungay KM. Ware JE. Measuring and monitoring health-related quality
25. Alonso J. Antó JM. Moreno C. Spanish version of the Notthingham He alth Profile: Translation and preliminary validity. Am \& Public Health 1990; 80: 704-708.

26. Badia X, Alonso J. Adaptación de una medida de la disfunción relacioada con la eniermedad: la version española del Sickness impact Profile. Med Clin (Barc) 1994; 102: 90-95

27. Alonso J. Prieto L, Anto JM. La versión española del «SF-36 Health Survey» (Cuestionario de Salud SF-36): un instrumento para la medida o Anderson JP. Bush JW. Berry CC Classiying 104: 171-7/6 me and quality of life evaluation. Med Care 1986: 24:454-469. EuroQol ${ }^{8}$ Group. EuroQol ${ }^{3}-$ a new facility for the measurement of healt related quality of life. Health Policy 1990; 16: 199-208.

30. Aaronson NK. Methodologic issues in assessing the qual ity of life of can-

of life and pharmacoeconomics in clinical trials $(2 \circ \mathrm{ed}$ ) Filadelfia: Lippincott-Raven 1996.

2. Guidelines for the pharmaceutical industry on preparation of submissions to the pharmaceutical benefits advisory committee lincluding submissions involving economic analysis). Camberra, Australla. Commonweat

Department or Healh. Housing and Communty Services, agosto de 1992. Ontario guidelines for economic analysis of pharmaceutical products. Real Decreto 271/1990, de 23 de febrero, sobre la reorganización de la mano. Boletin Oficiai del Estado n. ${ }^{\circ} 53$, de 2 de marzo de 1990; 6.086 6.087

Real Decreto 83/1993, de 22 de enero, por el que se regula la selección .

6. Udvarhelyi S Colditz GA, Rai A, Epstein AM Cost effectiveness and cos benefit analyses in the medical literature. Ann Intern Med 1992; 116.

37. Lee JT, Sánchez LA. Interpretation of cost-effective and soundness of economic evaluations in the pharmacy literature. Am I Hosp Pharm Sacristán JA Soto J Gark

dies: utilization of a heck-list An Evaluation of pharmacoeconomics stuEisenberg JM. Clinical economics. A guide to economic analyses of clintcal practices. JAMA 1989; 262: 2.879-2.886.

Barr JT, Schumacher GE. Applying decision analysis to pharmacy mana-
gement and practice decisions. Top Hosp Pharm Manage 1994; 13: $61-71$. 


\section{Capítulo 3}

Carrera-Hueso FJ. Estudios de calidad de vida relacionados con la salud. Farm Clín 1997; 14: 98-109. 


Revisiones
restudios de calidad de vida
relacionada con la salud

Farm Clin 1997; Vol. 14 n. ${ }^{\circ}$

\section{Resumen}

El objetivo es revisar los estudios de calidad de vida y de satisfacción de los pacientes así como las aplicaciones de los mismos.

Para medir la calidad de vida relacionada con la salud se utilizan cuestionarios, genéricos y específicos. Las propiedades psicométricas de los cuestionarios son: fiabilidad, validez, sensibilidad y aceptabilidad por los pacientes y personal sanitario.

Las aplicaciones de Ios cuestionarios son: monitorizar la salud de una población en general, en evaluaciones de política sanitaria, en ensayos clínicos y en la práctica clínica diaria.

Otros estudios, la satisfacción de los pacientes con el cuidado sanitario, aportan información muy útil.

Un nuevo campo de investigación, la productividad laboral de los pacientes, está emergiendo. Queda por dilucidar el alcance que pueda tener la utilización de estos estudios en eí quehacer diario de los profesionales sanitarios y su influencia en el sistema sanitario.

Calidad de vida relacionada con la salud. Farmacoeconomía. Satisfacción de los pacientes. Psicometría.

\author{
Abstract \\ The aim is to review the patient quality of life \\ and satisfaction studies, as well as their \\ applications. \\ Both generic and specific questionaires are \\ used to measure health-related quality of life. \\ The psychometric properties of the \\ questionnaires are: reliability, validity, \\ sensitivity and acceptability by patients and \\ health personnel. \\ The applications of the questionnaires are: \\ monitoring the health of a population in \\ general, in health policy appraisals, in clinical \\ trials, and in daily clinical practice. \\ Other studies, such as patient satisfaction with \\ health care, provide highly useful information. \\ A new field of research, the labour \\ productivity of patients, is emerging. \\ It remains to be seen to what extent these \\ studies may be used in the daily practice of \\ health professionals and what impact they may \\ make on the health system.
}

Health-related quality of life. Pharmacoeconomics. Patient satisfaction. Psychometrics.

\section{INTRODUCCION}

Tradicionalmente la medicina estaba más preocupada por sanar o paliar el efecto de las enfermedades que en mejorar el bienestar social de los pacientes. Para evaluar sus resultados utilizaba tres indicadores: mortalidad, morbilidad y el coste. Estos indicadores también servían para la toma de decisiones farmacoterapéuticas y su posterior monitorización (1).

Si tenemos en cuenta la definición de salud dada por la Organización Mundial de la Salud (2): "estado completo de bienestar físico, mental y social, no solamente la ausencia de la enfermedad", estos indicadores no son útiles ni

Este trabajo ha sido realizado gracias al premio DIDACTOR'93, otorgado por la revista Farmacia Clínica en colaboración de los Laboratorios GLAXO, S.A. 
Resultados y discusión

para monitorizar el resultado de nuestras actuaciones ni para disminuir el coste total de los tratamientos farmacológicos.

Kozma y Col. (3) diseñaron un modelo gráfico para comprender el complicado proceso de la toma de decisiones farmacoterapéuticas en el que no solo se miden los resultados clínicos del tratamiento de los pacientes sino también los resultados económicos y humanísticos del mismo. El modelo se describe en la Fig. 1. Los resultados clínicos son los derivados de una enfermedad o tratamiento; morbilidad y mortalidad. Los resultados económicos son aquellos que comparan los costes y las consecuencias de las distintas alternativas terapéuticas y los humanísticos son las consecuencias de la enfermedad sobre el estatus funcional del paciente o medidas sobre la calidad de vida en varias dimensiones.

El modelo también contempla la existencia de una serie de intermediarios o modificadores del tratamiento que pueden afectar a los resultados como por ejemplo el cumplimiento y las reacciones adversas del tratamiento. Recientemente Johnson y Bootman (4) han estimado el coste en los EE. UU. de la morbilidad y mortalidad debido dos con la utilización de medicamentos definidos por Helper y Strand (5), fue de 76,6 billones de dólares, imputándose el mayor gasto a las hospitalizaciones provocados por estos problemas.

Los autores concluyen que los problemas relacionados con la utilización de medicamentos pueden considerarse como una "enfermedad de comportamientos", la cual puede prevenirse con la utilización de servicios y controles sanitarios (4)

Según Donabedian (6) la calidad de los servicios sanitarios depende de la técnica, considerada como el conjunto de habilidades y conocimientos, y de las relaciones interpersonales, contemplándose la opinión de los pacientes acerca de sus tratamientos. Este autor considera la existencia de una conexión entre estructuras, procesos y resultados sanitarios (Fig. 2) (6-7).

Solamente podemos aumentar la calidad de los servicios sanitarios o mejorando los procesos a través de programas de garantía de calidad o aumentando la probabilidad de mejorar los resultados utilizando los estudios de calidad de vida, entendiendo estos resultados como los efectos de los cuidados sanitarios en los pacientes y poblaciones (6)

La farmacoeconomía es la descripción y el análisis de los costes del tratamiento con fármacos

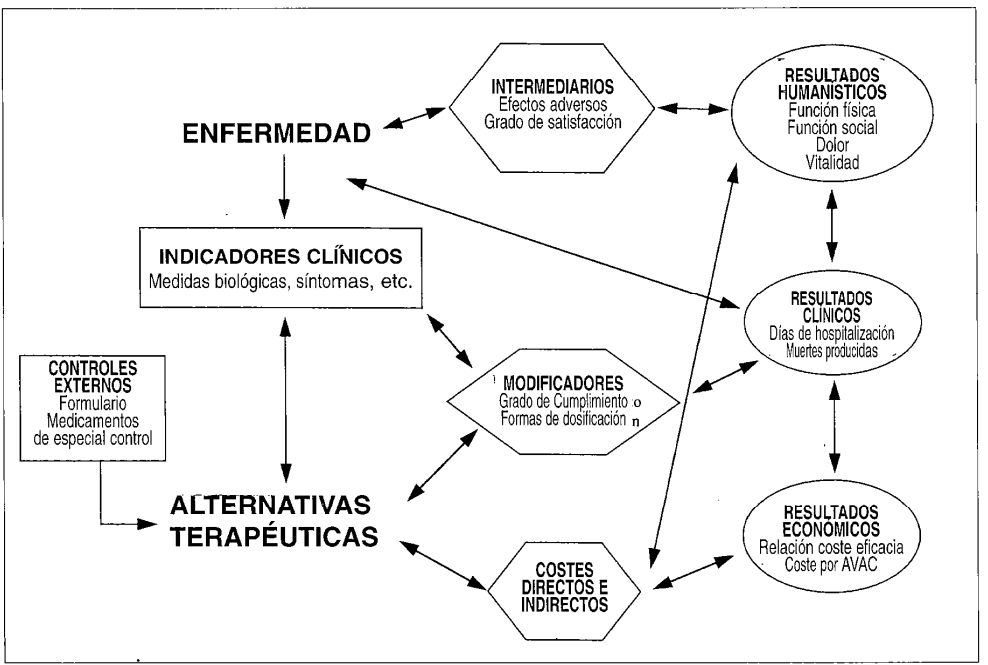

Fig. 1. Modelo de resultados 
Resultados y discusión

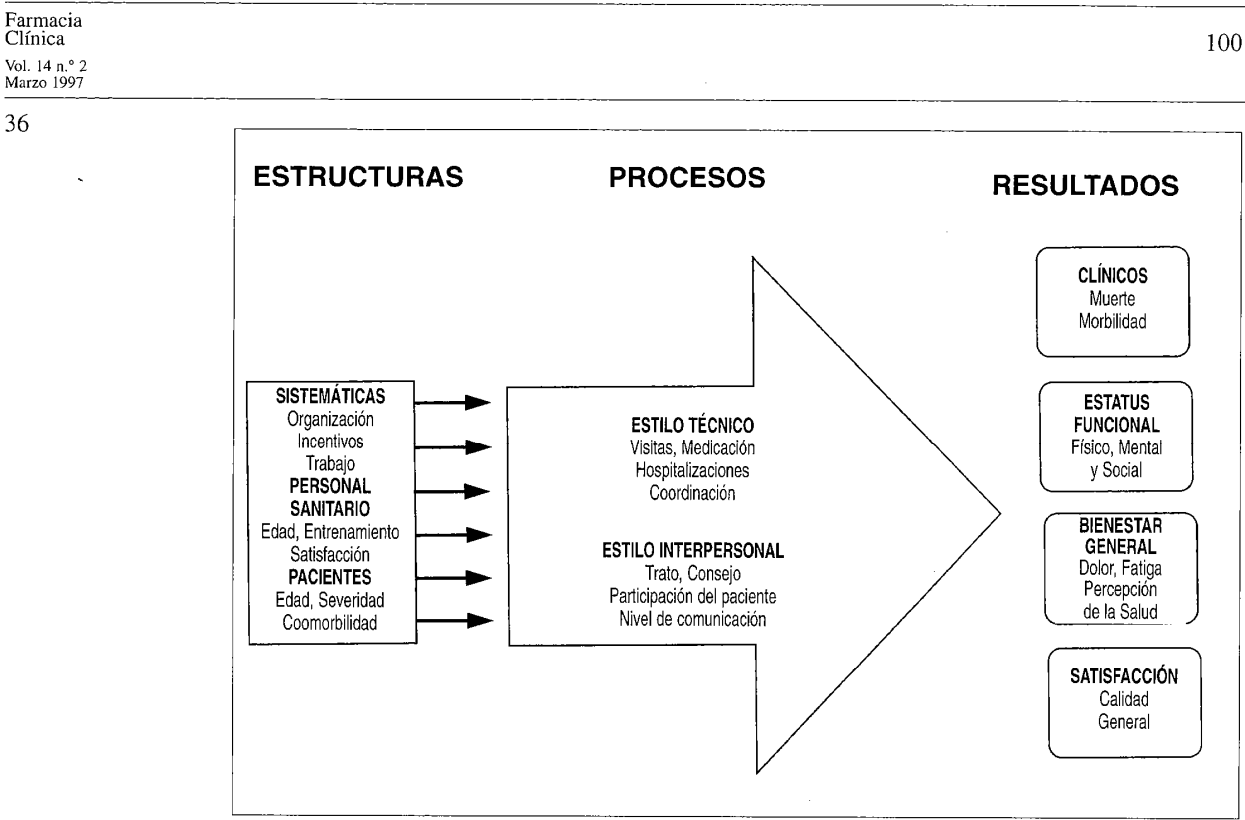

Fig. 2. Transcurso de la enfermedad.

en los sistemas de salud y en la sociedad (8). La farmacoeconomía se engloba dentro de la evaluación económica de tecnologías sanitarias. Los estudios farmacoeconómicos identifican, miden y comparan los costes (ej: recursos consumidos) y las consecuencias (clínicas, económicas y humanísticas) de los productos farmacéuticos y servicios (9).

Estos estudios incluyen además de las evaluaciones farmacoeconómicas tradicionales como los análisis coste-efectividad, minimización de costes, coste-beneficio y coste-utilidad; los estudios de coste de una enfermedad, los análisis de decisión y las evaluaciones humanísticas (912). Los tipos de evaluaciones humanísticas incluyen: las preferencias y satisfacción de los pacientes y los estudios de calidad de vida relacionada con la salud (CVRS).

En 1988, Ellwood (13) describió el modelo de la gestión de los resultados de los cuidados sanitarios y propuso medir sistemáticamente en la practica clínica diaria el grado de funcionamiento y de bienestar de los pacientes.

Cada vez existe un mayor interés en conocer grado de bienestar y satisfacción de los pacientes con el cuidado medico, es decir conocer la opinión del paciente acerca de su tratamiento, la cual puede diferir substancialmente de los profesionales sanitarios (14).
Un estudio revelo que entre el 66 y el $75 \%$ de los pacientes adultos nunca o raramente eran preguntados por sus limitaciones para realizar sus actividades diarias (15), como consecuencia los médicos no están bien informados acerca del bienestar y del estatus funcional de sus pacientes. También se ha puesto de manifiesto que los médicos tienden a no detectar las habilidades funcionales de sus pacientes, especialmente las relacionadas con sus actividades sociales (16)

Grossman (1) argumenta que las razones para valorar los resultados sanitarios son: definir evaluar y mejorar la efectividad del servicio prestado; controlar los costes de los servicios superfluos o inapropiados y documentar el valor del cuidado sanitario.

El objetivo del presente trabajo es revisar los estudios de calidad de vida y de satisfacción de los pacientes con el cuidado sanitario; así como las aplicaciones de los mismos.

\section{CALIDAD DE VIDA}

La vida tiene dos dimensiones: cantidad y calidad (14). La salud es uno de los doce dominios de la vida considerados cuando evaluamos la calidad de vida total de las personas. Los res- 
Resultados y discusión

\begin{tabular}{ll}
\hline Carmacia & 10
\end{tabular}

tantes dominios identificados por Campbell (17) son: la nación, la comunidad, la autoestima, la vida familiar, la relación con los amigos, la economía, la cducación, el hogar, el pueblo barrio donde vivimos, el trabajo y si estamos o no casados.

Por lo que deberemos considerar la calidad de vida relacionada con la salud como un termino diferente a la calidad de vida. Es decir, el concepto de calidad de vida se refiere a todos los aspectos de nuestras vidas como por ejemplo, donde vivimos, que empleo tenemos, etc; en cambio, la CVRS solo se refiere a aquellos aspectos de nuestras vidas que son dominados o están influenciados por nuestra salud personal (18).

La CVRS es el valor asignado a la duración de la vida modificado por la disminución, estado funcional, percepciones y oportunidades sociales debido a una enfermedad, accidente, tratamiento o política (19). Los pacientes son la principal fuente de información en los estudios de CVRS.

Para medir la CVRS se utilizan cuestionarios (también llamados instrumentos, test o perfiles) que evalúan los diferentes estados de salud. Los cuestionarios pueden ser genéricos o. específicos de una enfermedad o condición $(10,18,20)$

\section{CUESTIONARIOS GENERICOS}

Los primeros cuestionarios aplicados en la práctica clínica, como el indice de Karnofsky (21), fue para valorar las limitaciones físicas en pacientes gravemente enfermos. Aunque estos pacientes gravemente enfermos. Aunque estos
instrumentos carecían de la metodología de la psicometría en su diseño, los datos obtenidos con los mismos eran válidos y reproducibles. Estos instrumentos fueron el punto de partida para el desarrollo de cuestionarios o perfiles de salud que ahora se utilizan en investigación y en la clínica (22)

Los cuestionarios modernos, es decir aquellos que están basados en métodos de construcción de escalas, aparecieron en los años setenta. Estos cuestionarios son multidimensionales y valoran la salud física, social y psicosocial de los pacientes. Algunos de estos cuestionarios son el Sikness Impact Profile (23) o la Escala del Bienestar (Quality of Well-Being) (24), los cuales son todavía ampliamente utilizados.

Instrumentos más recientemente han sido desarrollados con el objeto de aumentar su aceptabilidad por parte de los pacientes y profesio- nales sanitarios, con un menor número de preguntas que sus antecesores y sus preguntas se centran no sólo en las cualidades negativas de la salud sino también en las positivas. Ejemplos de estos últimos instrumentos son: Duke de estos últimos instrumentos son: Duke
University of North California Health Profiles (25), Nottingham Health Profile (26), Medical Outcomes Study 36-Item Health Survey (SF36) (22) y el Euroqol (27)

En la Tabla 1 se describe la clasificación y principales características de los cuestionarios genéricos más utilizados $(14,22-28)$.

Los instrumentos genéricos evalúan todos los aspectos relevantes de la salud y no están in fluenciados por el tipo de enfermedad, raza, edades, etc. Son multidimensionales, es decir, están diseñados para aplicarlos en diferentes enfermedades, intervenciones médicas y pueden utilizarse en una amplia gama de poblacioden utilizarse
nes $(10,14,18)$

Los cuestionarios genéricos son aplicables tan to a la población en general como a los pacientes con cualquier tipo de enfermedad, su principal limitación es intercultural Algunos de estos instrumentos han sido adaptados para su utilización en la población española (29-31) En el Anexo I se muestra una parte de la versión española del SF-36 (31).

Los test genéricos evalúan al menos cuatro conceptos del estado de la salud: la función física, la función mental, la función de relaciones sociales y del trabajo, y las percepciones generales de la salud. En la Tabla 2 se describe y definen los distintos conceptos de la salud recogidos en los cuestionarios $(18,32)$.

Las preguntas para valorar la función física de los pacientes se centran en las limitaciones fácilmente percibidas por ellos mismos o por observadores externos. La medida del funcionamiento físico de los pacientes no sólo deben estar dirigidas a sus limitaciones físicas sino más bien deben incluir preguntas en el test referidas a la posibilidad de realizar actividades diarias así como a la satisfacción con su condición física (32).

Como se recoge en la Tabla 2 la función de las relaciones sociales sociales y del trabajo es un importante aspecto que deben rccogcr los cuestionarios que valoran la CVRS. Normalmente aunque se refiere a este concepto conjuntamente, su valoración se realiza separadamente en función social y funcionamiento del rol. La función social recoge 1 frecuencia de los contactos interpersonales y la 
Resultados y discusión

Tabla 1. Conceptos y características de los cuestionarios generales de calidad de vida relacionada con la salud.

Cuestionarios

\begin{tabular}{|c|c|c|c|c|c|c|c|c|c|}
\hline & QWB & SIP & HIE & NHP & QLI & COOP & Euroqol & DUKE & SF-36 \\
\hline \multicolumn{10}{|l|}{ Conceptos } \\
\hline Función física & $\bullet$ & $\bullet$ & $\bullet$ & $\bullet$ & $\bullet$ & $\bullet$ & - & $\bullet$ & $\bullet$ \\
\hline Función social & $\bullet$ & $\bullet$ & $\bullet$ & $\bullet$ & $\bullet$ & $\bullet$ & - & $\bullet$ & $\bullet$ \\
\hline Rol trabajo & $\bullet$ & $\bullet$ & $\bullet$ & $\bullet$ & $\bullet$ & $\bullet$ & - & $\bullet$ & $\bullet$ \\
\hline Distress psicológico & & $\bullet$ & $\bullet$ & $\bullet$ & $\bullet$ & $\bullet$ & - & $\bullet$ & $\bullet$ \\
\hline Percepción general de la salud & & & $\bullet$ & - & $\bullet$ & $\bullet$ & - & $\bullet$ & $\bullet$ \\
\hline Dolor corporal & & $\bullet$ & $\bullet$ & $\bullet$ & & $\bullet$ & - & $\bullet$ & $\bullet$ \\
\hline Vitalidad (energía) & • & & • & - & & & & $\bullet$ & $\bullet$ \\
\hline Bienestar psicológico & & & - & & & & & $\bullet$ & $\bullet$ \\
\hline Dormir & & $\bullet$ & & $\bullet$ & & & & $\bullet$ & \\
\hline Función cognitiva & & $\bullet$ & & & & & & $\bullet$ & \\
\hline Calidad de vida & & & - & & & $\bullet$ & & & \\
\hline Pronóstico sobre la salud & & & & & & $\bullet$ & & & $\bullet$ \\
\hline \multicolumn{10}{|l|}{ Caracteristicas" } \\
\hline Método de administración & A, E & $\mathrm{A}, \mathrm{E}$ & $\mathrm{A}, \mathrm{E}$ & $\mathrm{A}, \mathrm{E}$ & $\mathrm{A}, \mathrm{E}$ & $A, E$ & $\mathrm{~A}, \mathrm{E}$ & $\mathrm{A}, \mathrm{E}$ & $\mathrm{A}, \mathrm{E}$ \\
\hline Número de preguntas & 107 & 136 & 86 & 38 & 5 & 9 & 9 & 17 & 36 \\
\hline Tipo de puntuaciones & I & $\mathrm{P}, \mathrm{I}, \mathrm{SP}$ & $P$ & $\mathrm{P}$ & I & $P$ & I & $\mathrm{P}, \mathrm{I}$ & P, SP \\
\hline \multicolumn{10}{|c|}{$\begin{array}{ll}\text { QWB }=\text { Quality of Well-Being Scale }(1973) & \text { COOP = Dartmouth Function Charts (1987) } \\
\text { SIP = Sickness Impact Profile (1976) } & \text { EUROQOL = European Quality o Life Index (1990) } \\
\text { NIE = Health Insurance Experience surveys (1979) } & \text { DUKE = Duke Health Profile (1990) } \\
\text { NHP = Nottingham Health Profile (1980) } & \text { SF-36 = Medical Outcomes Study 36 Item Short-Form Health } \\
\text { QLI = Quality of Life Index (1981) } & \text { Survey (1992) }\end{array}$} \\
\hline
\end{tabular}

naturaleza de los mismos; en cambio, el funcionamiento del rol recoge las actividades desarrolladas por los pacientes relacionados con e trabajo ya sea remunerado o no como en el caso de las amas de casa o de los estudiantes. Aunque las mayores limitaciones del rol son debidas a problemas físicos, también se han detectado en ausencia de los mismos, por lo que este concepto también tiene un componente mental (33).

La ansiedad y la depresión son recogidos por los instrumentos generales para valorar la salud mental, pero es necesario para realizar una valoración de la CVRS de este concepto más comprensiva y recoger también el bienestar psicológico, la función cognitiva y el control emocional de los pacientes.

Aunque los cuestionarios genéricos han sido utilizados para el "screening" de alguna enfer medad mental (ej.: depresión) (32), es importante destacar que los mismos no pueden ser considerados herramientas diagnósticas de ninguna enfermedad mental.

La valoración de la percepción general de la salud es nęcesaria porque permiten recoger las diferencias individuales en diferentes percep- ciones difíciles de valorar, como el dolor, el grado de preocupación causado por la enfermedad, etc. (32).

\section{CUESTIONARIOS ESPECIFICOS}

Los instrumentos específicos asumen que cada condición médica tiene un específico resultado y además deben medirse las funciones afectadas por esa condición. Estos cuestionarios valoran sólo aquellos aspectos importantes de una enfermedad o síntomas o estados mentales o a un determinado grupo de pacientes (ej.: oncológicos, ancianos) $(9,10,18,20)$

Diferentes áreas han sido investigadas previamente con instrumentos específicos como el funcionamiento sexual y emocional, náuseas y vómitos, dolor, ansiedad y depresión, asma, rinitis y epilepsia (34-36)

$\mathrm{Su}$ principal inconveniente es que solamente pueden aplicarse en los casos para los que fueron diseñados y no permiten comparaciones entre enfermedades, sin embargo tienen la ventaja de presentar una alta sensibilidad a los cambios $(10,18,20)$ 
Resultados y discusión

\begin{tabular}{|c|c|c|c|}
\hline \multicolumn{4}{|l|}{$\begin{array}{l}\text { Farmacia } \\
\text { Clínica } \\
\text { Vol. } 14 n^{\circ} 22 \\
\text { Marzo } 1997 \\
\end{array}$} \\
\hline \multirow{7}{*}{\multicolumn{2}{|c|}{39}} & & \\
\hline & & & Tabla 2. Conceptos generales de salud (24). \\
\hline & & Concepto & Definición \\
\hline & & $\begin{array}{l}\text { Función física } \\
\text { Limitaciones físicas } \\
\text { Habilidades físicas } \\
\text { Días encamado } \\
\text { Dolor corporal } \\
\text { Bienestar físico }\end{array}$ & $\begin{array}{l}\text { Limitaciones que impiden el cuidado personal, la movilidad y las actividades físicas } \\
\text { Habilidad para realizar las actividades diarias } \\
\text { Confinamiento en la cama debido a problemas de salud } \\
\text { Grado de intensidad, frecuencia, y duración del dolor corporal y las limitaciones de } \\
\text { las actividades debidas al mismo } \\
\text { Evaluación personal de la condición física }\end{array}$ \\
\hline & & $\begin{array}{l}\text { Función de relaciones s } \\
\text { Contactos } \\
\text { interpersonales } \\
\text { Recursos sociales } \\
\text { Funcionamiento (rol) }\end{array}$ & $\begin{array}{l}\text { ociales y del trabajo } \\
\text { Frecuencia de visitas a amigos y frecuencia de contactos telefónicos durante } \\
\text { específicos periodos } \\
\text { Cantidad y calidad del ambiente social que les rodea } \\
\text { Limitaciones debidas a la salud que les impide realizar actividades normales } \\
\text { relacionadas con el trabajo, los estudios o las tareas domésticas }\end{array}$ \\
\hline & & $\begin{array}{l}\text { Salud mental } \\
\text { Ansiedad/Depresión } \\
\text { Bienestar psicológico } \\
\text { Control emocional } \\
\text { /comportamientos } \\
\text { Función cognitiva }\end{array}$ & $\begin{array}{l}\text { Sentimientos de ansiedad, depresión, nerviosismo, malhumor, descorazonamiento } \\
\text { Frecuencia e intensidad de afectos positivos generales } \\
\text { Control del comportamiento, pensamientos y sentimientos durante específicos } \\
\text { periodos } \\
\text { Orientación temporal y espacial, memoria, atención y alerta }\end{array}$ \\
\hline & & $\begin{array}{l}\text { Percepciones generales } \\
\text { Salud actual } \\
\text { Salud futura }\end{array}$ & $\begin{array}{l}\text { de la salud } \\
\text { Autovaloración de la salud actual } \\
\text { Expectaciones acerca de la salud en el futuro }\end{array}$ \\
\hline
\end{tabular}

Para medir la CVRS en los pacientes con tratamientos farmacológicos, Aaronson (37) propuso la utilización de un test genérico y otro específico, de esta manera el específico proporcionar información más detallada y será más sensible a los cambios, y el genérico permitirá comparar los resultados con otros estados de enfermedad.

\section{UTILIDADES}

Los estudios coste-utilidad miden los efectos a través de una unidad que integra la cantidad y calidad de vida, años de vida ajustados por calidad (AVAC) (9-12). Los AVAC se obtienen calculando los años de vida ganados ponderándolos con la calidad de vida por técnicas que miden los estados de salud basadas en preferencias o utilidades.

Estas técnicas son $(10,11)$ : escalas de categorías, juego estándar, estimación de la magnitud, equivalencia temporal y equivalencia de personas. Los estados de salud obtenidos pueden agregarse obteniendo una puntuación global, a partir de la cual se calculan los AVAC para los estudios coste-utilidad.

Los AVAC también pueden calcularse por métodos indirectos utilizando las medidas de utilidad o escalas de salud, las cuales permiten obtener un índex o índice a partir del cual se obtienen los AVAC. Las escalas más comúnmente utilizadas son: la matriz de Rosser y Kind (38) la escala del bienestar (24) y el Euroqol (27)

\section{PROPIEDADES PSICOMÉTRICAS}

La psicometría es la ciencia que usa test o escalas para medir atributos individuales. En el campo de la salud se utiliza para trasladar los sentimientos, comportamientos y valoraciones de los pacientes en datos cuantificables (18)

Hay dos propiedades psicométricas que cualquier instrumento de medida de la CVRS debe poseer: ser válidos y fiables. Además las escalas de medida deben ser sensibles a los cambios y tener un grado de aceptación suficiente por los clínicos y pacientes (32)

\section{Fiabilidad}

La fiabilidad es la relación entre la varianza de la verdadera medida y la varianza de la medida observada. Es decir, la fiabilidad se refiere a la consistencia, estabilidad o reproducibilidad de la medida obtenida en diferentes administra 
Resultados y discusión

40

ciones del instrumento cuando todas las condiciones no cambian en el tiempo (39).

La fiabilidad es expresada en decimales entre valores de 0 y 1 , cuyos valores más cercanos a la unidad reflejan menores posibilidad de cambios. Por ejemplo, un valor de 0.75 , indica que el $75 \%$ de la varianza observada en el dato es varianza cierta y que el $25 \%$ de la varianza observada es el resultado de un cambio. Si no existen cambios ni en la varianza ni en el error de randomización la fiabilidad estimada sería la unidad.

Los dos métodos para valorar la fiabilidad descritos en la literatura son test-retest y el de consistencia interna $(9,10,20)$. Debido a que la salud y la enfermedad son procesos dinámicos, e incluso muchos estudios lo que pretenden es medir los cambios observados en la CVRS de los pacientes en el tiempo, los métodos test-retest tienen poco valor para estimar la fiabilidad $(32,39)$.

El método de consistencia interna que indica la extensión que un instrumento está libre del error de randomización, es el más adecuado cuando valoramos la CVRS. La estimación de la fiabilidad es a menudo obtenida por la estimación del coeficiente alfa, calculado utilizando el coeficiente alfa de Cronbach (40).

La fiabilidad está influida por el número de preguntas del test y los valores de fiabilidad aceptables para la comparación de grupos deben ser superiores a 0.50 o 0.70 . El cambio para comparación a nivel individual las estimaciones de la fiabilidad deben ser valores superiores a $0.90(41)$

\section{Validez}

Un instrumento de medida es válido si mide aquello que queremos medir, en nuestro caso la CVRS. Por ejemplo, antiguamente se pensaba que a mayor circunferencia de la cabeza, mayor grado de inteligencia tenía el individuo, por lo que el metro sería un instrumento de medición válido. Más tarde se comprobó que esta hipótesis era errónea, así que este instrumento de medir la inteligencia (el metro) no era válido.

Tres tipos de validez debe cumplir un instrumento cuando valoramos la CVRS, éstos son: de contenido, de construcción y criterio (1820,42 )

La validez. de contenido nos indica si el concepto es capturado o no por las preguntas del test y en qué grado. Una escala con una buena validez de contenido es aquella que cubre todos los aspectos del concepto que pretende medir. Para establecer la validez de contenido de los instrumentos se comparan las preguntas del test con un estándar definido que tiene una aceptación general.

La validez de construcción se refiere a si el instrumento es capaz de medir diferencias entre grupos previamente establecidos (ej.: edad, sexo). La validez divergente y convergente son dos tipos de validez de construcción. Así para la validez divergente la correlación entre los resultados del instrumento de ambos grupos conocidos que difieren substancialmente entre si será baja. En cambio, la correlación para la validez convergente será alta para grupos que no difieren entre si. Por ejemplo, los resultados de un instrumento que mide la actividad física y la movilidad, tendrán una validez convergente; pero si además mide la ansiedad y la depresión tendrá una validez divergente respecto a los resultados de la actividad física.

La validez criterio requiere la comparación de las puntuaciones de la escala respecto a un estándar de oro, que no existe para la medición de CVRS. Sin embargo, puede utilizarse un estándar de oro relativo comparando un instrumento con mayor número de preguntas con otro con menor número de preguntas (22).

\section{Sensibilidad}

La sensibilidad es la característica de los instrumentos de CVRS menos estudiada (18). La sensibilidad es la capacidad del instrumento de medida de detectar diferencias de la CVRS en el tiempo clínicamente significativas. Debido a que la sensibilidad puede estar afectada por el número de opciones de respuestas o niveles que tiene cada pregunta, se ha propuesto utilizar un mayor número de niveles en cada pregunta para mejorar la sensibilidad de los instrumentos.

\section{Aceptabilidad}

Otra característica que deben cumplir los cuestionarios que miden la CVRS es que sean aceptados por los investigadores, clínicos y respondedores; esto es que sean fáciles de usar, administrar, puntuar e interpretar

Cuestionarios con un mayor número de preguntas tienen menor aceptabilidad por los pa- 
Resultados y discusión

$\begin{array}{lr}\text { Farmacia } & 105\end{array}$

cientes que otro con menos preguntas. Se tiende a obtener cuestionarios que tengan una fiabilidad aceptable y una menor carga de respuesta disminuyendo el número de preguntas (ej.: SF-36) (22).

\section{APLICACIONES DE LOS ESTUDIOS DE CALIDAD DE} VIDA

Los cuestionarios de CVRS pueden aplicars en monitorizar la salud de una población en general, en evaluaciones de política sanitaria, en ensayos clínicos y en la práctica clínica diaria $(14,42)$.

\section{Monitorización de la salud de una población}

La utilización de índices son difíciles de interpretar como indicadores de la salud de una población porque no reflejan las diferencias existentes en el acceso a los servicios ni otros factores que puedan influir en los estados de salud.

Así por ejemplo, las estadísticas de mortalidad son índices utilizados para comprobar el grado de salud de una sociedad, pero en realidad aportan una información sesgada referente a la calidad de la misma.

Con estudios de CVRS se puede conocer mejor tanto el grado como la calidad de salud de una población debido a que reflejan también los aspectos negativos de la salud así como el grado de accesibilidad a los servicios sanitarios por parte de los pacientes.

\section{Evaluación de la política sanitaria}

En una época de contención del gasto sanitario, ya no sólo interesa medir los resultados clínicos de un programa sanitario sino también los económicos y humanísticos (3). Estos estudios permiten demostrar reducciones en los costes sanitarios sin afectar a la calidad de los servicios prestados por las instituciones sanitarias.

Para que estos estudios sirvan de apoyo en la toma de decisiones requieren: métodos de valoración válidos, apropiado diseño del estudio y un alto nivel de precisión estadística en estimar los efectos sobre la salud.

Estos cuestionarios también son de utilidad en los estudios de coste-utilidad para el cálculo de los AVAC.como anteriormente habíamos indicado.

\section{Ensayos clínicos}

El interés por los estudios farmacoeconómicos han ido aumentando progresivamente en la última década de tal manera que la media de estuma década de tal manera que la media de esvariables económicas ascendió del $1.7 \%$ en 1988 al 23.7\% en 1994 (43). Esta tendencia parece que se mantendrá en el futuro, debido a que los laboratorios farmacéuticos necesitan demostrar en un mercado altamente competitivo el valor de sus productos.

De la misma manera, en la investigación clínica con medicamentos se incorporarán variables como el bienestar y satisfacción de los pacientes con el cuidado sanitario así como de la CVRS (15), permitiendo a la industria farmacéutica demostrar el valor de los medicamentos frente a sus inmediatos competidores.

\section{En la práctica clínica diaria}

A diferencia de los estudios FE, los distintos grupos de investigadores han ido desarrollando sus propios cuestionarios, lo que impide la comparación con otros estudios publicados (42). Hay un número de cuestiones metodológicas que deben considerarse en la valoración gicas que deben considerarse en la valoración de estudios de CVRS en la práctica clínica:
¿Como qué instrumento debe utilizarse (general o específico)? ¿Qué es más útil medir el índex o los perfiles? ¿Qué dimensiones deben medirse y cuáles no? ¿La relativa importancia de las dimensiones medidas en el estado global de CVRS? ¿La fiabilidad y validez de los instrumentos? $(10,18,20)$.

La utilización clínica de los resultados de la CVRS es incipiente y se han aplicado principalmente en el tratamiento y monitorización de enfermedades crónicas (44) y en ensayos clínicos de medicamentos (8)

Con la utilización de alta tecnología, base de datos y escáners, permitirá que el proceso y el análisis de la información obtenida en los cuestionarios sea más rápido obteniendo resultados inmediatos, punto clave para su aceptación en la práctica clínica diaria (14).

\section{SATISFACCION DE LOS}

\section{PACIENTES}

Como indicamos anteriormente, a la hora de valorar la calidad de los servicios sanitarios debemos tener presente que tanto las estructuras. los procesos y los resultados influyen positiva o 
Resultados y discusión

\begin{tabular}{|c|c|}
\hline $\begin{array}{l}\text { Farmacia } \\
\text { Clínica } \\
\text { Vol. } 14 \text { n.022 } \\
\text { Marzo } 1997\end{array}$ & \\
\hline 42 & $\begin{array}{l}\text { negativamente sobre los servicios prestados. } \\
\text { La satisfacción de los pacientes es considerado } \\
\text { un resultado en sí mismo (Fig. 1) (6-7). Es de- } \\
\text { cir, en términos puramente económicos, se tra- } \\
\text { ta de la evaluación de los bienes y servicios por } \\
\text { los consumidores de los mismos (en nuestro } \\
\text { caso los pacientes) como un camino para mo- } \\
\text { nitorizar la calidad del producto. }\end{array}$ \\
\hline
\end{tabular}

En la última década se ha utilizado la evaluación del consumidor o la satisfacción de los pacientes para evaluar la calidad del cuidado sanitario. Es similar en algunos aspectos a la valoración de la CVRS de los pacientes ya que de alguna manera medimos un resultado final del cuidado de los mismos.

Las mismas técnicas psicométricas descritas anteriormente son utilizadas para obtener la información y valorar su validez y fiabilidad en este tipo de estudios. Sin embargo, la información requerida a los pacientes o consumidores es única y muy diferente a la que es preguntada en los perfiles o escalas para valorar la CVRS de los pacientes.

Tanto los informes como las valoraciones de los pacientes son utilizados en este tipo de estudios. Los informes simplemente no requieren de una valoración subjetiva por parte del entrevistado; un ejemplo sería: ¿Cuánto tiempo tuvo que esperar para que le recibiera su doctor? En cambio las valoraciones obtenidas tienen un componente subjetivo, por ejemplo: ¿Tuvo que esperar demasiado tiempo para le atendiese su médico?

El número de cuestionarios disponibles que

valoran el grado de satisfacción de los pacientes con el cuidado médico es amplio y los atributos comúnmente valorados incluyen (45-47): el conocimiento y habilidades del equipo multidisciplinar que atiende al paciente, la calidad de la comunicación médico-paciente, si el trato recibido por el personal sanitario es humano, $y$ el grado de implicación de los pacientes en su cuidado.

Cuatro características en este área por las que son preguntados los pacientes son csencialcs para utilizar esta información a posteriori. Estas son: accesibilidad al cuidado sanitario, continuidad del cuidado recibido, comprensibilidad del cuidado y si el cuidado sanitario es integral. En la Tabla 3 se resumen los objetivos, requerimientos e intervenciones para los diferentes estamentos involucrados en el cuidado sanitario (46).

El conocimiento de la opinión de los pacientes se ha utilizado frecuentemente en la evaluación de los servicios hospitalarios (ej.: Join Commission norteamericana) (46), pero empieza a ser importante también a otros niveles de la atención sanitaria como la atención primaria.

\section{OTROS ESTUDIOS QUE MIDEN} LOS RESULTADOS SANITARIOS

El absentismo laboral anualmente está asociado a una importante pérdida económica para el conjunto de la sociedad. Lo que intentan de-

\begin{tabular}{|c|c|c|c|c|}
\hline & $\begin{array}{l}\text { Administradores } \\
\text { hospitalarios }\end{array}$ & Investigadores & Personal sanitario & Pacientes \\
\hline Objetivos & $\begin{array}{l}\text { Marketing } \\
\text { Relaciones públicas } \\
\text { Gestión } \\
\text { Satisfacción pacientes }\end{array}$ & $\begin{array}{l}\text { Métodos rigurosos } \\
\text { Validación } \\
\text { Aumento conocimiento } \\
\text { Medir los cambios }\end{array}$ & $\begin{array}{l}\text { Incrementar salarios } \\
\text { Promociones } \\
\text { Satisfacer a pacientes } \\
\text { Resolver problemas }\end{array}$ & $\begin{array}{l}\text { Mejorar cuidado futuro } \\
\text { Mejorar la utilización de recursos } \\
\text { Participar en el cuidado }\end{array}$ \\
\hline Requerimientos & $\begin{array}{l}\text { Barato } \\
\text { Informativo } \\
\text { Uso efectivo } \\
\text { Rápida disposición datos }\end{array}$ & $\begin{array}{l}\text { Sensibles } \\
\text { Sin errores } \\
\text { Válidos } \\
\text { Fiables }\end{array}$ & $\begin{array}{l}\text { Rápida disposición datos } \\
\text { Relevantes } \\
\text { Informativos } \\
\text { Efectivos }\end{array}$ & $\begin{array}{l}\text { Fáciles de completar } \\
\text { Comprensibles } \\
\text { Valiosos por el tiempo utilizado }\end{array}$ \\
\hline Productos & $\begin{array}{l}\text { Comentarios } \\
\text { Resultados }\end{array}$ & $\begin{array}{l}\text { Método de investigación } \\
\text { Resultados estadísticos }\end{array}$ & $\begin{array}{l}\text { Comentarios } \\
\text { Satisfacción con el servicio } \\
\text { prestado }\end{array}$ & $\begin{array}{l}\text { Cuestionario completado } \\
\text { Cambios en el cuidado debido } \\
\text { a las respuestas }\end{array}$ \\
\hline Intervenciones & $\begin{array}{l}\text { Cambios en las decisiones } \\
\text { institucionales } \\
\text { Responder a } \\
\text { - comentarios negativos }\end{array}$ & $\begin{array}{l}\text { Evaluar servicios } \\
\text { Incorporar unidades } \\
\text { de medida distintas }\end{array}$ & $\begin{array}{l}\text { Diseñar soluciones } \\
\text { Implantar otros servicios }\end{array}$ & Proveer opinión \\
\hline
\end{tabular}


Resultados y discusión

\begin{tabular}{|c|c|}
\hline \multicolumn{2}{|l|}{$\begin{array}{l}\text { Farmacia } \\
\text { Clínica }\end{array}$} \\
\hline 43 & $\begin{array}{l}\text { mostrar este tipo de estudios es la influencia de } \\
\text { un tratamiento farmacológico sobre el absen- } \\
\text { tismo laboral o sobre la productividad laboral } \\
\text { de los pacientes. }\end{array}$ \\
\hline & $\begin{array}{l}\text { Aunque tradicionalmente se preguntaba a los } \\
\text { pacientes en ciertos cuestionarios sobre la pér- } \\
\text { dida de horas laborables y los días encamados } \\
\text { a causa de su enfermedad, recientemente di- } \\
\text { versos instrumentos han sido desarrollados pa- } \\
\text { ra medir como afecta a la capacidad de trabajo } \\
\text { de las personas diversas enfermedades cróni- } \\
\text { cas. Esto significa que la capacidad de trabajo } \\
\text { o productividad de las personas o grupo deter- } \\
\text { minado de personas debe ser considerado co- } \\
\text { mo un resultado sanitario en si mismo y más } \\
\text { concretamente en un resultado humanístico } \\
\text { del cuidado sanitario (Fig. } 1 \text { ). }\end{array}$ \\
\hline & $\begin{array}{l}\text { Estos nuevos instrumentos utilizan las técnicas } \\
\text { psicométricas descritas anteriormente y están } \\
\text { siendo desarrollados para medir las caracterís- } \\
\text { ticas tanto de la enfermedad de los pacientes } \\
\text { como de los requisitos necesarios por los pa- } \\
\text { cientes para cada situación laboral. Estas áreas } \\
\text { se encuentran actualmente en fase de investi- } \\
\text { gación (48). }\end{array}$ \\
\hline
\end{tabular}

\section{CONCLUSIONES}

Para conocer la eficiencia de los tratamientos farmacológicos es necesario conocer todos los resultados de los mismos (clínicos, económicos y humanísticos); lo que implica el conocimiento de los mismos sobre la CVRS de los pacientes.

Los pacientes son la principal fuente de información en los estudios de CVRS. La CVRS se mide por medio de cuestionarios, genéricos o específicos; algunos de los cuales también se pueden utilizar para el cálculo de los AVAC en los estudios coste-utilidad.

Los instrumentos deben ser: fáciles de usar, interpretar y administrar; aplicables a diferentes poblaciones; ser fiables, válidos y prácticos; deben incluir aspectos del bienestar de los pacientes; tener un amplio rango de medidas y ser comprensivos.

Los estudios de satisfacción de los pacientes sirven para conocer mejor la calidad del cuidado sanitario tanto en el ambiente hospitalario como en la atención primaria. El conocimiento de la productividad laboral de los pacientes está actualmente siendo un nuevo campo de investigación en este área.

Los cuestionarios de CVRS pueden aplicarse en monitorizar la salud de una población en ge. neral, en evaluaciones de política sanitaria, en ensayos clínicos y en la práctica clínica diaria. Queda por dilucidar el alcance que pueda tener la utilización de estos estudios en el queha-

cer diario de los profesionales sanitarios y su influencia en el sistema sanitario.

BIBUOGRAFIA

1. Grossman $\mathbf{J H}$. The outcomes movement and health care reform. Am J Helath-Syst Pharm 1995; 52 (Supp)
3). S6-11. 2. Basic documents: World Health Organization. Gene
Switzerland: World Health Organization; 1948.

3. Kozma CM, Reeder CE, Schulz RM. Economic, clinical, and humanistic outcomes: a planning model for 32.

4. Johnson JA, Bootman JL. Drug-related morbidity and mortality. A cost-of-illness model. Arch Intern Med 1995; 155:1949-56

5. Helper CD, Strand LM. Opportunities and responsabilities in pharmaceutical care. Am J Hosp Pharm 1990

6. Donabedian A. The quality of care: how can it be assessed? JAMA 1988; 260:1743-48.

7. Ferris KB, Kirking DM. Assesing the quality of pharmaceutical care II: applications of concepts of quality assessment from medical care. Ann Pharmacother 1993, 27:215-23.

8. Towsend JR. Postmarketing drug research and development. DICP 1987; 21:134-136

9. Bootman JL, Townsend RJ, McGhan WF. Principles of pharmacoeconomics. 2nd ed. Cincinnati: Harvey

10. Sacristán JA, Badia X, Rovira J, eds. Farmaeconomía evaluación economica de medicamentos. Editores médicos, Madrid, 1995.

11. Drummond MF, Stoddart GL, Torrance GW. Basic types of economic evaluation. In: Methods for the econoMedical Publications, 1987: 78.

12. Sánchez LA. Pharmaeconomic principles and methods: including pharmacoeconomics into hospital pharmacy practice. Hosp Pharm 1995; 29:1035-40.

13. Ellwood PM. Outcomes management: A technology of patient experience. N Engl J Med 1988; 318: 1549-56.

14. Ware JE. The status of health assessment 1994. Ann Rev Public Health 1995; 16:327-54.

15. Cramer JA, Scheyer RD, Mattson RH. Compliance declines between clinic visits. Ann Intern Med 1990; 150:1509-10.

16. Schor EL, Lerner DJ, Malspeis S. Physician's assessment of functional health status and well-being: The patient's perspective. Arch Intern Med 1995; 155:309-14.

17. Campbell A. The sense of well-being in America: re- 
Resultados y discusión

Farmacia
Clínica
Vol. $14 \mathrm{n}{ }^{\circ} 2$
Marzo 1997

108

44

18. Bungay KM, Ware JE. Measuring and monitoring health-related quality of life. Kalamazoo, MI: Upjohn, 1993.

19. Patrick DL, Erickson P. Health status and health policy. Allocating resources to health care. New York: Oxford University Press, 1993

20. Testa MA, Simonson DC. Assessment of quality-of-life outcomes. N Engl J Med 1996; 322:835-40.

21. Karnofsky DA, Burchenal JH. The clinical evaluation of chemotherapeutic agents in cancer. In: Macleod CM,
ed. Evaluation of chemotherapeutic agents. New York: Columbia Press 1949:191-205.

22. Ware JE Jr, Sherbourne CD. The MOS 36-Item ShortForm Health Survey (SF-36). Med Care 1992; 32:473482

23. Bergner M, Bobbit RA, Pollard WE, Martin DP, Gilson BS. The Sickness Impact Profile: Validation of a health Istatus measure. Med Care 1976; 14:57-67.

24. Fanshel S, Bush JW. A health-status index and its application to health-services outcomes. Operations Res 1970; 18: 1021-66.

25. Parkerson GR, Broadhead WE, Tse CK. Comparison of the Duke Health Profile and the MOS Short-Form in healthy young adults. Med Care 1991; 29:679-83.

26. Hunt SM, McEwen J, McKenna SP. Measuring health status a new tool for clinicians and epidemiologists. J R

27. EuroQol ${ }^{\oplus}$ Group. EuroOol ${ }^{\oplus}$-A new facility for the measurement of health related quality of life. Health Policy 1990; 16:199-208.

28. Nelson EC, Landgraf JM, Hays RD, Wassan JH, Kirk JW. The COOP function charts: a system to measure patient function in physicians offices. In: Lipkin M Jr, ed. Functional Status Measurement in Primare Care. New York, NY: Springer-Verlag, 1990.

29. Alonso J, Antó JM, Moreno C. Spanish version of the Nothingham Health Profile: Translation and prelim nary validity. Am J Public Health 1990; 80:704-708.

30. Badia X, Alonso J. Adaptación de una medida de la difusión relacionada con la enfermedad: la versión espa-
ñola del Sickness Impact Profile. Med Clín (Barc) 1994; 102:90-95.

31. Alonso J, Prieto L, Antó JM. La versión española del "SF-36 Health Survey" (Cuestionario de Salud SF-36): 作

32. Ware JE Jr. Standards for validating health measures: definition and content. J Chron Dis 1987; 40:473-480.

33. Sherbourne CD, Stewart AL, Wells WB. Role fuctioning measures. In: Stewart $\mathrm{AL}$, Ware JE, eds.
Study approach. Durham, NC: Duke University Press:
1992: $205-19$

34. Patrick DL, Deyo RA. Generic and desease-specific Ineasures in assessing health status and quality of life. Med Care 1989; 27 (suppl):S217-32.

35. Juniper EF, Guyatt GH, Ferrie PJ, Griffith LE Measuring quality of life in asthma. Am Rev Respir Dis

36. Vickrey BG, Hays RD, Engel J Jr, Spritzer K, Rogers WH, Rausch R, Graber J, Brook RH. Outcome assessment for epilepsy surgery: the impact of measuring health-related quality of life. Ann Neurol 1995; 37(2): 158-

37. Aaronson NK. Methodologic issues in assessing the quality of life of cancer patients. Cancer 1991; 67:844-50.

38. Rosser R, Kind P. A scale of valuations of states on illness: Is there a social consensus? Int J Epidemiol 1978; $7: 347-58$.

39. Coons SJ, Kaplan RM. Quality of life assessment: understanding its use as an outcome measure. Hosp
Formul 1993; 28:486-98.

40. Cronbach LJ, Warrington WG. Time-limit tests: estimating their reliability and degree of speeding.

41. Nunnally JC. Psycometric theory, 2nd ed. New York, NY: McGraw-Hill, 1978.

42. Ware JE, Keller SD. Interpreting general health measures. In: Quality of life and pharmacoeconomics in clinical trials, 2nd Ed. Spilker B, ed. Philadelphia, PA Lippincott-Raven Publishers, 1996: 445-60.

43. Gagnon JP. A primer on pharmacoeconomics. Managed

44. Meyer KB. The outcomes of ESRD and its treatment Adv Renal Repl Ther 1995; 2:101-11.

45. Meterko M, Nelson EC, Rubin HA. Patient judgmen of hospital quality: report a pilot study. Med Care 1990 28(Suppl):S1-S56.

46. Dull VT, Lansky D, Davis N. Evaluating a patient satisfaction survey for maximum benefit. The Joint faction survey for maximm
Commission 1994; 20:444-53.

47. Brouard A, Daures JP, Jalabert A, Hansel S Hazebroucc G. Patient satisfaction questionnaire to evaluate continuing medical education. Paper presented to annual ASHP meeting. San Diego, CA. 1996 June $2-6$

48. Berndt ER, Finkelstein S, Greenberg P, Parsley RA, Verner J, Keller MB. Improvement in subjetive work performance after treatment of chronic depression. CA. 1996. June 2-6.

Recibido: 11-11-96. 
Resultados y discusión

\begin{tabular}{|c|c|c|c|c|}
\hline $\begin{array}{l}\text { Farmacia } \\
\text { Clínica } \\
\text { Vol. } 14 n^{\circ}{ }^{2} 2 \\
\text { Marzo } 1997 \\
\end{array}$ & & & & 109 \\
\hline \multirow{14}{*}{45} & & & & \\
\hline & \multicolumn{4}{|c|}{ Anexo 1. Cuestionario "SF-36" sobre el estado de salud" } \\
\hline & \multicolumn{4}{|c|}{$\begin{array}{l}\text { Pregunta 3: Las siguientes preguntas se refieren a actividades o cosas que usted podría hacer en un día normal. } \\
\text { Su salud actual ile limita para hacer esas actividades o cosas? Si es así, ¿cuánto? } \\
\text { (marque un sólo número por cada pregunta) }\end{array}$} \\
\hline & Actividades & $\begin{array}{l}\text { Sí, } \\
\text { me } \\
\text { limita } \\
\text { mucho }\end{array}$ & $\begin{array}{c}\text { Sí, } \\
\text { me } \\
\text { limita } \\
\text { un poco }\end{array}$ & $\begin{array}{l}\text { No, no } \\
\text { me } \\
\text { limita } \\
\text { nada }\end{array}$ \\
\hline & $\begin{array}{l}\text { a. Esfuerzos intensos, tales como correr, levantar objetos } \\
\text { pesados, o participar en deportes agotadores }\end{array}$ & 1 & 2 & 3 \\
\hline & $\begin{array}{l}\text { b. Esfuerzos moderados, como mover una mesa, pasar la } \\
\text { aspiradora, jugar a los bolos o caminar más de } 1 \text { hora }\end{array}$ & 1 & 2 & 3 \\
\hline & c. Coger o llevar la bolsa de la compra & 1 & 2. & 3 \\
\hline & d. Subir varios pisos por la escalera & 1 & 2 & 3 \\
\hline & e. Subir un solo piso por la escalera & 1 & 2 & 3 \\
\hline & f. Agacharse $o$ arrodillarse & 1 & 2 & 3 \\
\hline & g. Caminar un kilómetro o más & 1 & 2 & 3 \\
\hline & h. Caminar varias manzanas (varios centenares de metros) & 1 & 2 & 3 \\
\hline & i. Caminar una sola manzana (unos 100 metros & 1 & 2 & 3 \\
\hline & j. Bañarse o vestirse por sí mismo & 1 & 2 & 3 \\
\hline
\end{tabular}

Pregunta 8: Durante las 4 últimas semanas, ¿hasta qué punto el dolor le ha dificultado su trabajo habitual (incluido el trabajo fuera de casa y las tareas domésticas)?

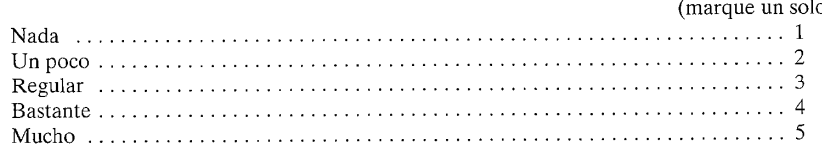

* Copyright $^{\oplus} 1993$ New England Medical Center Hospitals, Inc.

All rights reserved.

(IQOLA SF-36 Spanish (Spain) Versión 1.3) 


\section{Capítulo 4}

Carrera-Hueso FJ. Calidad de vida en un paciente epiléptico. Farm Clín 1997; 14: 74-89. 
Resultados y discusión

\begin{tabular}{|c|c|c|}
\hline $\begin{array}{l}\text { Farmacia } \\
\text { Clínica } \\
\text { vol. } 14 \text { n.2 }\end{array}$ & Originales & 74 \\
\hline 10 & & \\
\hline
\end{tabular}

Farm Clin 1997: Vol. 14 n. ${ }^{\circ}$ I

\section{Resumen}

En este trabajo se describe como afecta la medición de la calidad de vida relacionada con la salud (CVRS) en la monitorización y tratamiento de un paciente epiléptico en la práctica clínica diaria.

La selección del paciente fue retrospectiva y el cuestionario utilizado fue el Short Form-36 Healt Survey (SF-36). El método de administración fue el de autoadministrado supervisado por el entrevistador. Las respuestas fueron transcritas por un escáner; midiendo la calidad de las mismas. El perfil de la CVRS del paciente se conoció por tres métodos comparativos: población adulta, población de pacientes epilépticos y con la media de las administraciones previas.

Los resultados muestran que la salud mental fue más afectada que la salud física y estuvieron en consonancia con la situación clínica del paciente. La medición de la CVRS en la práctica clínica aporta nueva información a los clínicos y puede servir de soporte en la toma de decisiones farmacoterapéuticas.

Calidad de vida relacionada con la salud. Epilepsia. Short Form36 Health Survey (SF-36). Decisiones farmacoterapéuticas.

\begin{abstract}
In this study we describe how measurement of health-related quality of life (HROL) affects the monitoring and treatment of an epileptic patient in daily clinical practice.

The selection of the patient was retrospective and the questionnaire used was the Short Form-36 Health Survey (SF-36). Interviewersupervised self-completion was the method of administration used. The answers were transcribed by scanner and their quality measured. The profile of the patient's HRQL was ascertained by three comparative methods: adult population, epileptic patient population, and with the mean of the previous administrations.

Results show that mental health was more affected than physical health and they were in keeping with the patient's clinical situation. In clinical practice the measurement of HRQL supplies clinicists with new information and may provide supportive grounds for making pharmacotherapeutic decisions.
\end{abstract}

Health-related quality of life. Epilepsy. Short Form-36 Health Survey (SF-36).

Pharmacotherapeutic decisions.

\section{INTRODUCCION}

La epilepsia es una enfermedad crónica caracterizada por descargas eléctricas originadas en un foco del cerebro que afectan sincrónicamente a las neuronas corticales con o sin convulsiones. Esta alteración es medible mediante electroencefalograma (EEG) (1). La epilepsia implica la existencia de crisis recurrentes las cuales varían ampliamente según el grado de severidad, aparición, causa, consecuencias y tratamiento. Su incidencia en los EE.UU. es de 120 por 100.000 habitantes, es decir $300 \mathrm{mi}$ nuevos casos al año y se estima que al menos el $8 \%$ de la población padecerá una crisis en su vida (2). Las causas desencadenantes de las crisis pueden ser mecánicas (ej.: trauma cerebral, neoplasias), metabólicas (ej.: hiperventila- 
Resultados y discusión

Farmacia
Clíica
Vol.1 14 n. 22
Marro 1997

Vol. $14 \mathrm{n}^{\circ}{ }^{2}$
Marzo 1997

12

ción), tóxicas (ej.: infección), hereditarias, idiopáticas y por la supresión brusca de fármacos (ej.: alcohol, antiepilépticos, antipsicóticos) (1).

En la última década se han realizado avances tanto en el tratamiento como en el diagnóstico de las crisis epilépticas (1). Distintas técnicas de imagen diagnósticas, como la tomografía computarizada (TAC), tomografía de emisión de positrones (PET) y la resonancia magnética han contribuido junto al EEG a diagnosticar correctamente la enfermedad y localizar los focos causantes permitiendo una clasificación de los tipos de epilepsia.

Los objetivos del tratamiento de la enfermedad son controlar y reducir la frecuencia de las crisis (idealmente a cero), minimizar los efectos secundarios del tratamiento (quirúrgico o médico) y prevenir comportamientos y sentimientos o emociones aberrantes $(1,3)$.

Recientemente un mayor número de pacientes, seleccionados estrictamente, son sometidos a cirugía para corregir o disminuir la frecuencia de sus crisis, con un éxito del 80-90\% de los mismos, teniendo mejor pronóstico los que tienen un foco localizado en el lóbulo temporal (4). Los pacientes candidatos a cirugía son aquellos que cumplen los siguientes requisitos: diagnóstico cierto de epilepsia, fallo del tratamiento farmacológico y definición del síndrome electroclínico $(1,4)$.

El tratamiento farmacológico es de por vida excepto cuando hay evidencia de que el paciente no ha tenido crisis al menos durante 5 años (1). La monoterapia es de elección inicialmente y permite controlar a muchos pacientes pero un $20 \%$ de los mismos son refractarios al tratamiento, por lo que necesitan más de un fármaco $(1,5)$. En los últimos años se han incorporado nuevos medicamentos para el tratamiento de la enfermedad refractaria a los fármacos convencionales con resultados modes tos, pero debido a su baja toxicidad y a su alta especificidad están teniendo una gran aceptación (5). Todavía no se ha valorado la seguridad a largo plazo de estos tratamientos. El tratamiento con medicamentos debe ser seguro y eficaz, por lo que es necesario por un lado la monitorización de sus concentraciones sanguíneas, asegurar el cumplimiento del tratamiento por parte de los pacientes y controlar las manifestaciones tanto clínicas como biológicas de los efectos secundarios $(1,3,5)$

Tanto el tratamiento como la propia enfermedad afectan al comportamiento social del paciente lo que origina una pérdida de producti- vidad y escolarización (6). En consecuencia, el paciente prefiere no cumplir el tratamiento porque los efectos secundarios del mismo superan al miedo a la crisis y a la muerte (3). El pánico a la muerte debe considerarse como una entidad patológica distinta y por lo tanto debe tratarse separadamente (1). El incumplimiento del tratamiento en los pacientes epilép ticos adultos es aproximadamente del $50 \%$, lo que sin duda refleja la necesidad de educarles acerca de su enfermedad y tratamiento $(7,8)$.

En 1990 un estudio reveló que entre el 66 y el $75 \%$ de los pacientes adultos nunca o raramente eran preguntados por sus limitaciones para realizar sus actividades diarias (9), como consecuencia los médicos no están bien informados acerca del bienestar y del estatus funcional de sus pacientes. También se ha puesto de manifiesto que los médicos tienden a no detectar las habilidades funcionales de sus pacientes, especialmente las relacionadas con sus actividades sociales (10). Más de la mitad de los pacientes epilépticos preguntados en el es tudio Roper (6) se sentían infelices y el 59\% de los mismos comentó que no tenían más remedio que aceptar las frecuencias de sus crisis y los efectos secundarios de su tratamiento.

Los resultados en el tratamiento de los pacientes no son solamente los clínicos (derivados d una enfermedad o tratamiento; morbilidad mortalidad) sino también los resultados económicos (comparan los costes y las consecuencias de las distintas alternativas terapéuticas) y humanísticos (consecuencias de la enfermedad sobre el estatus funcional del paciente o medidas sobre la calidad de vida en varias dimensiones) del mismo (11). Cada vez existe un mayor interés en conocer el grado de bienestar y satisfacción de los pacientes con el cuidado médico, es decir conocer la opinión del paciente acerca de su tratamiento, la cual puede diferir substancialmente de los profesionales sanitarios (12).

La vida tiene dos dimensiones: cantidad y calidad. En pacientes crónicos la calidad de vida e un factor determinante de su cuidado $(12,13)$. La calidad de vida relacionada con la salud (CVRS) es el valor asignado a la duración de la vida modificado por la disminución, estado funcional, percepciones y oportunidades sociales debido a una enfermedad, accidente, tratamiento o política (14). La CVRS es medible y cuantificable mediante cuestionarios o instrumentos, que pueden ser genéricos o específicos de una enfermedad o condición $(12,13)$. Los instrumentos genéricos evalúan todos los aspectos relevantes de la salud y no están influenciados por el tipo de enfermedad, raza, 
Resultados y discusión

$\begin{array}{lr}\text { Farmacia } & 77 \\ \text { Clínica } & \end{array}$

13

edades, etc. Son multidimensionales, es decir, están diseñados para aplicarlos en diferentes enfermedades o intervenciones médicas y pueden utilizarse en una amplia gama de poblaciones. La CVRS proporciona una nueva información a los clínicos que como demuestran diversos estudios puede afectar al proceso de decisiones farmacoterapéuticas (15-19). Los cuestionarios de CVRS pueden aplicarse en monitorizar la salud de una población en general, en evaluaciones de política sanitaria, en ensayos clínicos, en la monitorización y mejora de los resultados de los cuidados sanitarios y en la práctica clínica diaria (17). Para medir la CVRS en los pacientes con tratamientos farmacológi$\cos$, Aaronson (20) propuso la utilización de un test genérico y otro específico, de esta manera el específico proporcionará una información más detallada y será más sensible a los cambios, y el genérico permitirá comparar los resultados con otros estados de enfermedad.

Existen puntos de consenso entre los investigadores para medir la CVRS en la epilepsia (3) a) hay que conocer la opinión del paciente acerca de su tratamiento; b) los conceptos de salud que deben ser valorados como mínimo son: funcionamiento físico, limitaciones debidas a problemas físicos y emocionales, depresión/ansiedad y bienestar; y c) las medidas específicas y generales de la salud son necesarias para valorar comprensivamente la CVRS de los pacientes epilépticos.

Diversos cuestionarios que miden la CVRS se han utilizado en pacientes epilépticos adultos (21-23). Wagner y col. (18) demostraron que los resultados obtenidos con el Short Form-36 Health Survey (SF-36) en estos pacientes eran menores en seis de los ocho dominios que los obtenidos en la población adulta sana sin condición crónica preexistente: limitaciones del rol debidas a problemas físicos, limitaciones de rol debidas a problemas emocionales, salud mental, vitalidad, función social y percepción de la salud general. Además también definieron los parámetros psicométricos (13) de esta población, los cuales pueden servir para la comparación de resultados en el tratamiento de estos pacientes $(12,13,17-19)$.

Pocos estudios publicados muestran la utilidad de medir la CVRS en la toma de decisiones farmacoterapéuticas de un paciente individual posiblemente debido a que los clínicos no están todavía familiarizados con la interpretación de los resultados ya que la medición de CVRS es una ciencia incipiente (12). Cabe destacar el estudio realizado por Meyer y col. (19) en el cual describen la aplicación de los resultados del
SF-36 en el tratamiento de dos pacientes con insuficiencia renal crónica sometidos a sesiones de diálisis en régimen ambulatorio. Ningún estudio hasta la fecha se ha realizado para demostrar como afecta la medición de la CVRS en el tratamiento de un paciente epiléptico.

La medición de la CVRS en la monitorización y seguimiento del tratamiento de un paciente individualizado nos aporta información que puede ayudar en la toma de decisiones farmacoterapéuticas (3).

El objetivo de este estudio es comprobar como afecta la medición de la CVRS de manera rutinaria en la monitorización y tratamiento de un paciente epiléptico en la práctica clínica diaria

\section{METODOS}

Retrospectivamente seleccionamos un paciente para cuantificar y valorar los resultados de su estatus de salud a través del tiempo utilizando un instrumento genérico, el Short Form-36 Health Survey (24)

Los criterios de selección del paciente fueron: 1) diagnosis de epilepsia refractaria de difícil control; 2) que hubiese sido sometido a cirugía durante el período del estudio; y 3) que se le hubiesen administrado el cuestionario al me nos diez veces.

El SF-36 mide el estado funcional y de satisfacción y bienestar del paciente a través de ocho conceptos de la salud: función física (FF), limitaciones del rol debidas a problemas físicos (RF), dolor corporal (DC), percepción de la salud general (SG), vitalidad/energía (VI), función social (FS), limitaciones del rol debidas a problemas emocionales (RE) y salud mental (SM) (24). Las ocho escalas del SF-36 pueden agruparse en las que valoran predominantemente la salud física (FF, RF, DC) y las que valoran principalmente la salud emocional mental (RE, SM); las restantes escalas (SG, VI FS) miden ambos conceptos de salud. Además el SF-36 mide mediante una pregunta, que no se utiliza para la puntuación de las escalas, los cambios de salud en el tiempo $(12,17,24)$.

El método de administración del instrumento fue el de autoadministrado por el paciente supervisado posteriormente por el entrevistador (25). El paciente fue aleccionado en las primeras visitas sobre como debe rellenar el cuestionario. El SF-36 fue respondido por el paciente durante el tiempo de espera de sus visitas médicas de revisión sin la influencia previa del en- 
Resultados y discusión

\begin{tabular}{|c|c|c|}
\hline $\begin{array}{l}\text { Farmacia } \\
\text { Clínica } \\
\text { Vol. } 14 \text { 11. }{ }^{\circ} 2 \\
\text { Marzo } 1997\end{array}$ & & 80 \\
\hline \multirow[t]{5}{*}{16} & $\begin{array}{l}\text { trevistador u otro profesional sanitario acerca } \\
\text { de los problemas de salud del mismo. Una vez } \\
\text { completado el test, el entrevistador preguntaba } \\
\text { al paciente si deseaba responder a aquellas } \\
\text { preguntas que no había contestado o si desea- } \\
\text { ba corregir aquellas preguntas en las que había } \\
\text { señalado más de una respuesta. Todos los re- } \\
\text { sultados del cuestionario son transformados a } \\
\text { una escala de } 0 \text { a } 100 \text {, el } 0 \text { indica el valor más } \\
\text { bajo posible y el } 100 \text { el valor máximo que se } \\
\text { puede obtener o el mejor estado de salud en } \\
\text { cada dominio (24). }\end{array}$ & $\begin{array}{l}\text { edificios. El resultado global de la calidad pro- } \\
\text { porcionado por el RT-2000 puede ser }(25,26) \text { : } \\
\text { excelente (las } 36 \text { preguntas son contestadas y } \\
\text { las respuestas fueron completamcntc consis- } \\
\text { tentes), satisfactoria (la mitad de las respuestas } \\
\text { fueron completadas para las ocho escalas y la } \\
\text { consistencia de las mismas fue del } 85 \text { al } 99 \% \text { ) y } \\
\text { problemática (menos de la mitad de las pre- } \\
\text { guntas fueron contestadas en cualquier escala } \\
\text { y/o su consistencia interna fue menor del } \\
85 \% \text { ). El RT-2000 también proporciona una ta- } \\
\text { bla indicando las principales limitacioncs de- } \\
\text { tectadas por las escalas en las diferentes admi- } \\
\text { nistraciones (actual, previa e inicial) (26). }\end{array}$ \\
\hline & $\begin{array}{l}\text { (Response Technologies, Inc., East Greenwich, } \\
\text { Richmond) (26); el cual las valora y puntúa en } \\
\text { términos de porcentajes por cada concepto de } \\
\text { salud. Los datos son almacenados en una base } \\
\text { de datos que el propio sistema utiliza poste- } \\
\text { riormente para realizar operaciones entre los }\end{array}$ & $\begin{array}{l}\text { En la Tabla } 1 \text { se describen las estimaciones esta- } \\
\text { dísticas para los pacientes epilépticos adultos } \\
\text { comparadas con una población adulta sana }(18) \text {. } \\
\text { La muestra de la población se ajustó según la } \\
\text { edad, sexo, raza y nivel cultural para ser compa- } \\
\text { rable a la muestra de pacientes epilépticos }(17,18) \text {. }\end{array}$ \\
\hline & $\begin{array}{l}\text { datos actuales con los obtenidos en anteriores } \\
\text { administraciones, previa e inicial. }\end{array}$ & \multirow{2}{*}{$\begin{array}{l}\text { Los valores del } 95 \% \text { del intervalo de confianza } \\
\text { (95\% IC) de cada escala del SF-36 nos indican } \\
\text { la variación que pueden tener los resultados } \\
\text { obtenidos para un paciente individual. Estos } \\
95 \% \text { IC los utilizaremos para comparar los re- } \\
\text { sultados de CVRS de nuestro paciente con los } \\
\text { obtenidos en la población de pacientes epilép- } \\
\text { ticos y en la población adulta sana a lo largo del } \\
\text { tiempo, lo que reflejará si esos cambios son sig- } \\
\text { nificativos o no respecto a la norma }(19,25) \text {. }\end{array}$} \\
\hline & $\begin{array}{l}\text { La calidad de los datos obtenidos se mide me- } \\
\text { diante dos indicadores: la consistencia de las } \\
\text { respuestas y el porcentaje de preguntas contes- } \\
\text { tadas (25). La consistencia interna de las res- } \\
\text { puestas está basado en una confrontación, dos } \\
\text { a dos, de } 15 \text { respuestas dadas al cuestionario, } \\
\text { obteniendo el índice de consistencia de las res- } \\
\text { puestas. Por ejemplo, un par de respuestas in- }\end{array}$ & \\
\hline & $\begin{array}{l}\text { consistentes serían que el paciente puede } \\
\text { andar una milla pero no una manzana de }\end{array}$ & $\begin{array}{l}\text { Para el cálculo del } 95 \% \text { IC se utilizaron las si- } \\
\text { guientes fórmulas (19): }\end{array}$ \\
\hline
\end{tabular}

Tabla 1. Estimaciones estadísticas del SF-36 para pacientes epilépticos adultos comparadas con una población adulta sana(18).

\begin{tabular}{|c|c|c|c|c|c|c|c|}
\hline Concepto & $\begin{array}{l}N^{\circ} \text { de } \\
\text { ítems }\end{array}$ & $\begin{array}{l}\mathbf{N}^{\circ} \text { de } \\
\text { niveles }\end{array}$ & $\begin{array}{l}\text { Tamaño de la } \\
\text { muestra* }\end{array}$ & Media* & $\begin{array}{l}\text { Desviación } \\
\text { standard* }\end{array}$ & Fiabilidad* & $\begin{array}{l}95 \% \text { Intervalo } \\
\text { de confianza }\end{array}$ \\
\hline Función física & 10 & 21 & $\begin{array}{c}148 \\
(641)\end{array}$ & $\begin{array}{c}89.25 \\
(93.51)\end{array}$ & 8.57 & 0.91 & $\begin{array}{c}5.04 \\
(1.16)\end{array}$ \\
\hline Rol-Físico & 4 & 5 & $\begin{array}{c}148 \\
(641)\end{array}$ & $\begin{array}{l}69.60 \\
(95.01)\end{array}$ & 15.59 & 0.90 & $\begin{array}{c}9.66 \\
(1.45)\end{array}$ \\
\hline Dolor corporal $\uparrow$ & 2 & 11 & $\begin{array}{c}148 \\
(641)\end{array}$ & $\begin{array}{c}82.36 \\
(87.65)\end{array}$ & 9.47 & 0.91 & $\begin{array}{c}5.57 \\
(1.37)\end{array}$ \\
\hline Salud general & 5 & 21 & $\begin{array}{c}148 \\
(641)\end{array}$ & $\begin{array}{l}59.67 \\
(82.11)\end{array}$ & 2.82 & 0.80 & $\begin{array}{c}2.47 \\
(1.20)\end{array}$ \\
\hline Vitalidad & 4 & 21 & $\begin{array}{c}148 \\
(641)\end{array}$ & $\begin{array}{l}53.47 \\
(67.84)\end{array}$ & 3.41 & 0.85 & $\begin{array}{c}2.59 \\
(1.55)\end{array}$ \\
\hline Función social & 2 & 9 & $\begin{array}{c}148 \\
(641)\end{array}$ & $\begin{array}{c}76.18 \\
(89.94)\end{array}$ & 7.82 & 0.85 & $\begin{array}{c}5.94 \\
(1.57)\end{array}$ \\
\hline Rol-Emocional & 3 & 4 & $\begin{array}{c}148 \\
(641)\end{array}$ & $\begin{array}{c}67.19 \\
(88.40)\end{array}$ & 9.18 & 0.73 & $\begin{array}{l}9.35 \\
(2.27)\end{array}$ \\
\hline Salud mental & 5 & 26 & $\begin{array}{l}148 \\
(641)\end{array}$ & $\begin{array}{c}61.28 \\
(79.55)\end{array}$ & 3.58 & 0.81 & $\begin{array}{c}3.06 \\
(1.33)\end{array}$ \\
\hline
\end{tabular}

"El primer núnhero es el valor estadístico para pacientes epilépticos. El número entre paréntesis indica el valor estadístico para la población

adulta sana.
$\dagger$ No hay diferencias significativas entre ambas poblaciones, adulta sana y pacientes epilépticos. 
Resultados y discusión

\begin{tabular}{|c|c|}
\hline \multicolumn{2}{|l|}{$\begin{array}{l}\text { Farmacia } \\
\text { Clínica } \\
\text { Vol. } 14 \mathrm{n}^{\circ} 2 \\
\text { Marzo } 1997\end{array}$} \\
\hline \multirow[t]{4}{*}{17} & [1] $\mathrm{SEM}=\mathrm{SD} \sqrt{ } 1-\mathrm{r}$ \\
\hline & [2] $95 \%$ IC $=1.96 \pm$ SEM \\
\hline & $\begin{array}{l}\text { siendo, } \mathrm{SEM}=\text { error estándar de la muestra; } \\
\mathrm{SD}=\text { desviación estándar de la muestra y } \mathrm{r}= \\
\text { coeficiente de fiabilidad. }\end{array}$ \\
\hline & $\begin{array}{l}\text { Utilizaremos los } 95 \% \text { IC de las escalas para las } \\
\text { poblaciones, adulta y de pacientes epilépticos } \\
\text { (18), para saber si los cambios producidos se } \\
\text { correlacionan con los eventos clínicos del pa- } \\
\text { ciente. Por ejemplo si la puntuación de la esca- } \\
\text { la del DC es } 75 \text {, la diferencia entre la media de } \\
\text { las poblaciones y la puntuación individual es } \\
\text { mayor que el } 95 \% \text { IC respecto a la población } \\
\text { de pacientes epilépticos y adulta }(87.65-75= \\
12.65>1.37 \text { y } 82.36-75=7.36>5.57 \text {, ver Tabla } \\
\text { 1) y diremos que la puntuación está por deba- } \\
\text { jo de la norma (25). }\end{array}$ \\
\hline
\end{tabular}

También podemos conocer el perfil de la calidad de vida del paciente comparando los datos obtenidos con los resultados de las administraciones previas. La comparación es entre los resultados del cuestionario en una administración y la media de los resultados de las administraciones previas. En la Fig. 1 se presenta un modelo gráfico en la cual se reflejan los resultados del cuestionario SF-36 en un momento dado y la media de administraciones anteriores. Las diferencias en las que no se superponen las desviaciones de la media y del resultado (a, b, c, d y e, Fig. 1) se calculan por la fórmula siguiente:

$$
\begin{gathered}
{[3] \mathrm{d}\left(\mathrm{X}_{\mathrm{i}-1}-\mathrm{i}\right)_{\mathrm{SC}}=/\left(\mathrm{X}_{\mathrm{i}-1}-95 \% \mathrm{IC}_{\mathrm{SC}}\right)-} \\
\left(\mathrm{i}+95 \% \mathrm{IC}_{\mathrm{SC}}\right) /=/\left(\mathrm{X}_{\mathrm{i}-1}-\mathrm{i}\right)-2 \times 95 \% \mathrm{IC}_{\mathrm{SC}} /
\end{gathered}
$$

siendo, $\mathrm{X}=$ Media de los resultados desdela visita 1 hasta la visita ( $\mathrm{i}-1) ; \mathrm{i}=$ resultado en la visita $\mathrm{i}$; $\mathrm{SC}=$ escalas. Al tratarse de la misma escala los $95 \%$ IC son los mismos para la media y el punto.

Si el resultado en cada escala del SF-36 de esta ecuación es negativo, significa que ambas desecuación es negativo, significa que ambas des-
viaciones se superponen; y si es positivo significa que las desviaciones de la media y el nuevo punto no se superponen, mostrando una diferencia entre ambos valores ( $\mathrm{a}, \mathrm{b}, \mathrm{c}, \mathrm{d}$ y e, Fig. 1 ).

\section{RESULTADOS}

Varón de raza blanca, 36 años ( $76 \mathrm{~kg}, 172 \mathrm{~cm}$ ), fumador y consumidor de alcohol, diagnosticado de epilepsia parcial compleja a los siete años de edad. Se inicia tratamiento con fenitoína suspendiéndose a los 6 años por la aparición de efectos secundarios (hiperplasia gingival)

Desde entonces se intentaron tratamientos con distintos antiepilépticos en diferentes combinaciones no consiguiéndose en ningún momento un control clínico adecuado. En 1988 presenta además alteraciones del ritmo sueño/

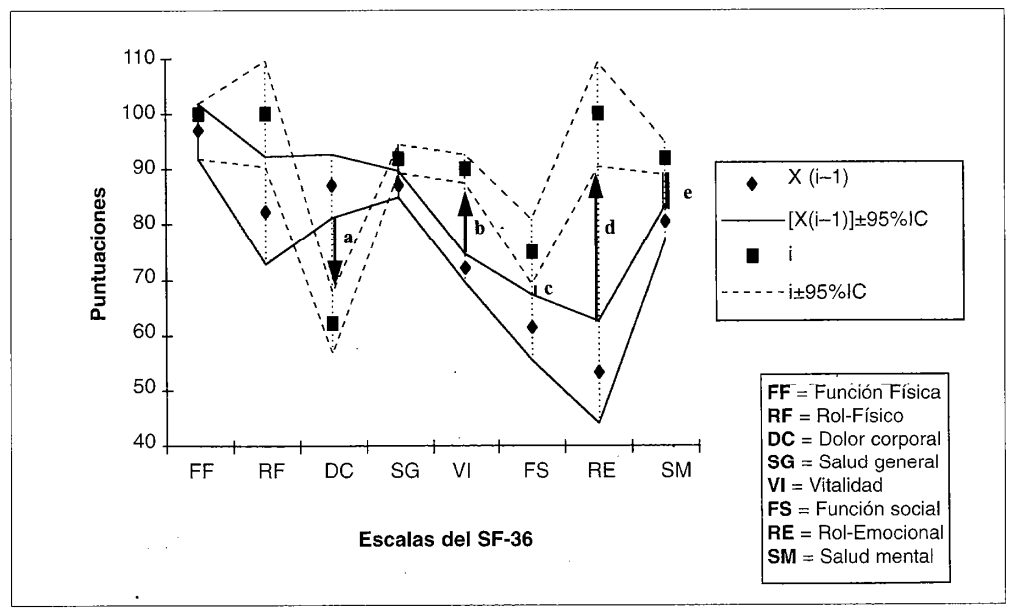

Fig. 1. Comparación de los resultados entre la última visita y la media de las visitas anteriores. 
Resultados y discusión

vigilia, diagnosticándose un síndrome de piernas inquietas o movimientos periódicos de sueño, sin relación con la enfermedad o su tratamiento. La Fig. 2 recoge el tratamiento del paciente desde el inicio de las administraciones del SF-36 y el número de crisis parciales simples y complejas.

Se realiza un seguimiento analítico del paciente cada dos meses que incluye: pruebas de función hepática, hematológicas y bioquímica completa. Los datos analíticos están dentro de los rangos normales durante este período.

En 1994, tras el fracaso del tratamiento médico, es sometido a cirugía realizándose una hipocampectomía. Tras la intervención quirúrgica se consigue un mejor control de las crisi tanto en cantidad como en el tipo de las mismas, registrándose menos crisis parciales complejas. Las pruebas neuropsicológicas muestran que su capacidad de seleccionar palabras y su memoria verbal no ha empeorado después de la cirugía y el paciente todavía tiene una buena función visual-espacial. La función neuropsíquica del paciente permanece estable desde la cirugía.

En la analítica de mayo 1996, se detecta un ligero incremento de sus niveles de colesterol total y triglicérido, de 201 y $198 \mathrm{mg} / \mathrm{dL}$ respectivamente, y un incremento de la GGT hepática (115 UI/L) relacionado posiblemente con los problemas etílicos del paciente. Los niveles sanguíneos de los medicamentos son monitorizados frecuentemente para ajustar sus dosificaciones y asegurar el cumplimiento del tratamiento (ácido valproico, carbamacepina). Se detectaron en varias ocasiones bajas concentraciones sanguíneas de ácido valproico que re- quirieron un aumento de la dosificación del mismo. El paciente no presentó ningún efecto secundario a los medicamentos.

La calidad de los datos obtenidos fue excelente: las respuestas fueron totalmente consistentes en todas las administraciones del SF-36 y el número de respuestas contestadas fue siempre del $100 \%$.

Los resultados de las escalas del cuestionario se muestran en las Figs. 3 y 4 . En estas figuras los resultados obtenidos de la CVRS del paciente son comparados con la norma de los pacientes epilépticos y una población adulta sana equivalente, ver Tabla 1.

Las puntuaciones del paciente en las escalas FF, SG y SM son superiores a la norma de los pacientes epilépticos. En relación a la población adulta sana, los resultados de FF son también superiores a la norma en todas las administraciones del cuestionario, en cambio tres de los resultados obtenidos en las escalas SG (vi sitas: $10 / 94,9 / 95$ y $12 / 95$ ) y SM (visitas: $1 / 95$ 9/95 y 12/95) están significativamente por debajo de la norma.

El dolor experimentado por el paciente viene reflejado en la escala DC (Fig. 3), el cual está por debajo significativamente tanto de la norma de los pacientes epilépticos como de la población adulta en la mayor parte del período estudiado. Todas estas visitas registran la misma puntuación en la escala DC, 77 , a excepción de la última visita (3/93) cuya puntuación es de 62

En la escala de VI, la visita del mes de enero de 1995 está por debajo de la norma de ambas poblaciones, epilépticos y adultos sanos, y la pun-

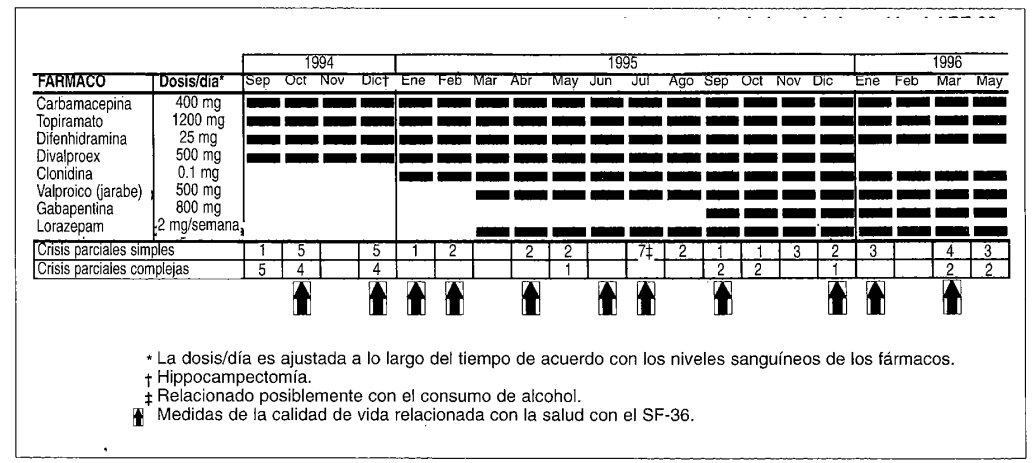

Fig. 2. Tratamiento y crisis epilépticas sufridas por el paciente desde la administración del SF-36. 


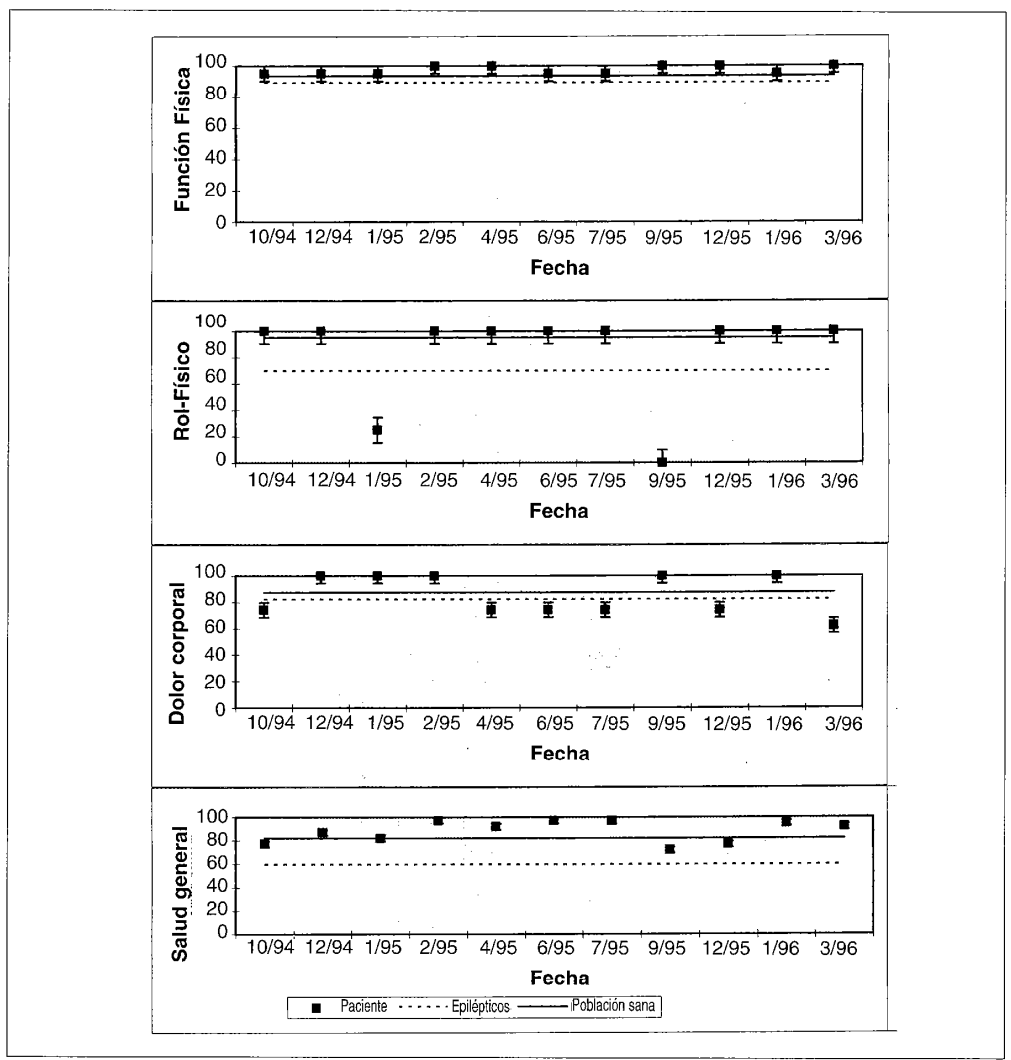

Fig. 3. Resultados de las escalas del SF-36 (I).

tuación registrada en la administración del 1/96 está significativamente solo por debajo de la población adulta.

Como muestra la Fig. 4 el FS del paciente está claramente por debajo de la población adulta sana, en cambio respecto a la población de pacientes epilépticos tan solo está por debajo de la norma en 4/95, 9/95 y en las tres primeras administraciones del SF-36. El RE está por debajo de la norma de ambas poblaciones, epilépticos y adultos sanos, en las tres primeras visitas, en septiembre del 95 y en enero del 96.

En la:Tabla 2 se recoge las diferencias de los resultados del cuestionario entre las medias de
las visitas anteriores $(\mathrm{X})$ y la última visita (i). A diferencia de la comparación entre los resulta- dos obtenidos del paciente y la población de pacientes, la comparación entre los propios resultados del paciente en el tiempo muestran una mejoría en varias escalas del FS en las diferentes administraciones y del SM en la última visita (1/96)

La escala del DC aunque disminuye su puntuación en casi todas las visitas respecto a la media anterior, las puntuaciones de las medias difieren menos de cinco puntos distribuyéndose los valores entre 84.7 y 89.6 .

Las diferencias superiores a diez puntos se registran en las escalas DC y VI en las administraciones del 3/96; y para la escala SF en 6/95. Las diferencias de 20,30 y hasta 40 puntos varía en la escala RE, cuyas medias varias am- 
Vol. 14 n. 02
Marzo 1997
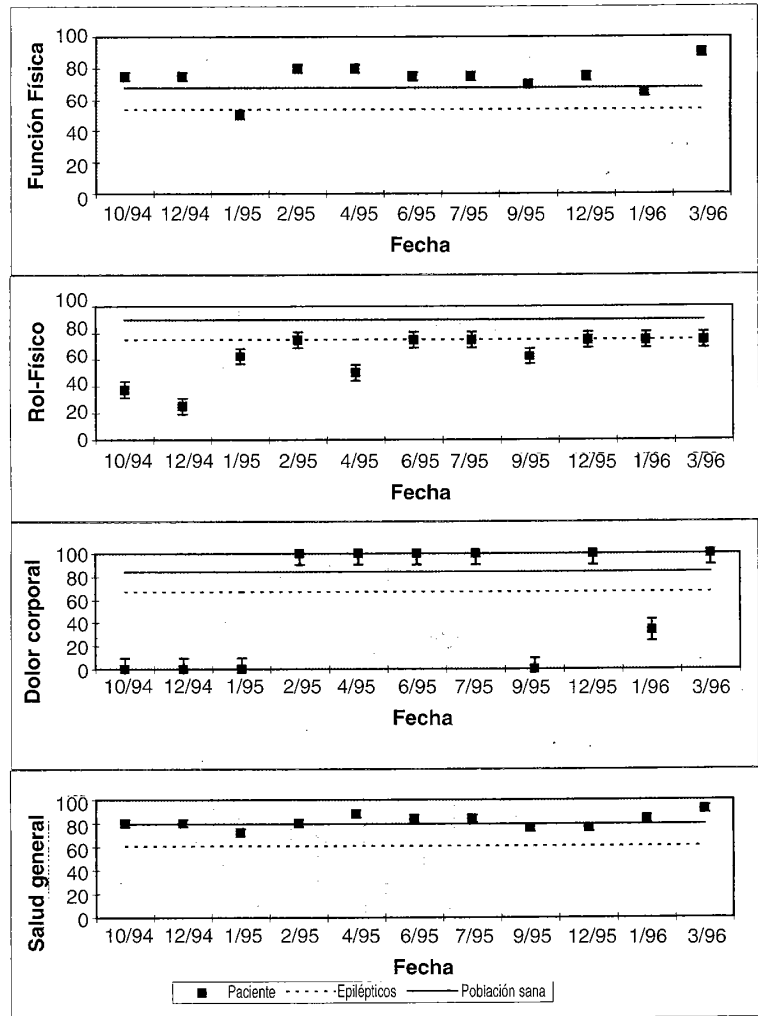

Fig. 4. Resultados de las escalas del SF-36 (II)

pliamente en el tiempo. El descenso más pronunciado respecto a su media, 70 puntos, se registra el 9/95 en la escala del RF.

Los resultados tan bajos registrados de CVRS inicialmente, en las tres primeras administraciones del instrumento para las escalas FS

$\mathrm{RE}$ están en consonancia con la dificultad de controlar las crisis epilépticas del paciente previa a la cirugía y las puntuaciones de la tercera visita para RF y VI son debidas a la situación debilitante causada por la misma.

Las puntuaciones obtenidas posteriormente la hippocampectomía se correlacionan con un mejor control la crisis del paciente aunque todavía persistían sus problemas del sueño justi- ficando posiblemente la disminución de la puntuación del FS (4/95) y DC

Las siete crisis parciales simples relacionadas con el consumo de alcohol etílico no tienen un claro impacto sobre la CVRS aunque la puntuación del DC se encuentre significativamente por debajo de la norma de los pacientes epilépticos y respecto a su media.

En septiembre de 1995 se registran puntuaciones en el suelo de la escala RF y RE, así como una disminución en FS pero no cambia respecto a su media, cuando empiezan a registrarse de nuevo mayor número de crisis parciales complejas de mayor duración y gravedad (pérdida de conciencia). Justificándose también la 
Resultados y discusión

Farmacia

Vol. $14 n^{\circ}{ }^{\circ} 2$
Marzo 1997

Tabla 2. Comparación entre los resultados del SF-36 en la última visita y la media de las visitas anteriores.

\begin{tabular}{|c|c|c|c|c|c|c|c|c|c|}
\hline \multicolumn{2}{|c|}{ Visitas } & \multicolumn{8}{|c|}{ Escalas del SF-36*† } \\
\hline Fecha & Diferencias & $\mathbf{F F}$ & $\mathbf{R F}$ & DC & SG & VI & FS & $\mathbf{R E}$ & SM \\
\hline $6 / 95$ & $\mathrm{X}_{5}-\mathrm{i}_{6}$ & n.c. & n.c. & $4.5 \downarrow$ & $5.1 \uparrow$ & n.c. & $13.1 \uparrow$ & $41.3 \uparrow$ & n.c. \\
\hline $7 / 95$ & $\mathrm{x}_{6}-\mathrm{i}_{7}$ & n.c. & n.c. & $1.9 \downarrow$ & $3.4 \uparrow$ & n.c. & $9.0 \uparrow$ & $31.3 \uparrow$ & n.c. \\
\hline 9/95 & $\mathrm{x}_{7}^{0}-\mathrm{i}_{8}^{\prime}$ & n.c. & $70.0 \downarrow$ & $3.7 \uparrow$ & $12.9 \downarrow$ & n.c. & n.c. & $38.4 \downarrow$ & n.c. \\
\hline $12 / 95$ & $\mathrm{X}_{8}-\mathrm{i}_{9}$ & n.c. & $2.6 \uparrow$ & $1.9 \downarrow$ & $5.7 \downarrow$ & n.c. & $5.3 \uparrow$ & $31.3 \uparrow$ & n.c. \\
\hline $1 / 96$ & $\mathrm{X}_{9}^{0}-\mathrm{i}_{10}$ & n.c. & $0.1 \uparrow$ & $3.3 \uparrow$ & $3.6 \uparrow$ & $2.6 \downarrow$ & $3.4 \uparrow$ & $3.6 \downarrow$ & n.c. \\
\hline $3 / 96$ & $\mathrm{X}_{10^{-i}}^{-\mathrm{i}_{11}}$ & n.c. & n.c. & $13.9 \downarrow$ & n.c. & $12.8 \uparrow$ & $1.9 \uparrow$ & $28.0 \uparrow$ & $5.5 \uparrow$ \\
\hline
\end{tabular}

* FF = función física; $\mathrm{RF}=$ rol-físico: $\mathrm{DC}=$ dolor corporal; $\mathrm{SG}$ = salud general; $\mathrm{VI}$ = vitalidad: $\mathrm{FS}$ = función social; $\mathrm{RE}=$ rol-emocional; $\mathrm{SM}$ $=$ salud menta.

$\uparrow$ n.c. = no cambia: $\uparrow=$ = Aumenta respecto a la media; $\downarrow$ = Disminuye respecto a la media.

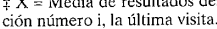

mayor disminución respecto a su media detectada en las escalas RF y RE.

A pesar de una puntuación significativamente más baja en la escala de RE en enero y de DC en marzo de 1996, en mayo se decide el cambio de tratamiento farmacológico del paciente para controlar o reducir tanto la frecuencia de las crisis epilépticas como sus problemas del sueño.

En resumen, la salud mental fue más afectada (FS, RE) que la salud física (FF, DC) del paciente, la cual fue buena y estuvo en consonancia con la situación clínica del paciente (RF) Las escalas que valoran ambos aspectos de la salud no sufrieron una variación clínica significativa.

\section{DISCUSION}

La CVRS depende de múltiples factores que pueden afectar a la salud, por lo que no depende únicamente de las condiciones patológicas que pueda presentar un paciente (17). De hecho consideramos que no es lo mismo partir de una muy baja CVRS en un paciente (ej.: angina de pecho incapacitante) que de una condición que le permita al paciente realizar sus actividades diarias (ej: diabetes mellitus no insulinodependiente). Además dentro de cada población especifica los pacientes se distribuyen en el techo (ceiling) o suelo (floor) de las escalas del instrumento (24).

Cada individuo tiene su propio patrón o "baseline" de CVRS diferente a todos los demás, debido al carácter personal de la vida. Es decir pacientes de la misma edad, sexo, patología, estatus sociocultural idénticos pueden diferi sus patrones de CVRS por la influencia de otros factores sobre la salud como: la organización politicosanitaria, la calidad del hospital, la preparación de los profesionales sanitarios que atienden al paciente, etc.

A pesar que la CVRS de un individuo varía con el tiempo (17-25), nosotros hemos definido el patrón de nuestro paciente en todas las escalas en base a referencias extrínsecas, comparación con otros pacientes, y a referencias intrínsecas, comparación con sus propios resultados. La definición del patrón individual nos servirá en la práctica clínica: a) para definir previamente el bienestar y el estatus funcional del paciente; b) para conocer el grado de mejoría o no, tanto cualitativa como cuantitativamente, del tratamiento; y c) para relacionar los cambios de la CVRS con la situación actual del paciente previa a la toma de decisiones farmacoterapéuticas.

Para comparar los resultados actuales del paciente con sus propios resultados nosotros hemos utilizado la media de las anteriores administraciones. Las limitaciones de este método comparativo reside en que la CVRS varía con el tiempo, por lo que deben definirse el número correcto de administraciones necesarias para formar la media con la que se va a comparar el resultado actual en cada escala del cuestionario y valorarse cual es el tiempo anterior válido para calcular la media en cada escala del SF-36.

Este caso también pone de manifiesto la menor utilidad del patrón interno o personal de paciente en las escalas que sufren grandes variaciones (por ejemplo la RE en nuestro paciente), que aquellas que presentan una mayor 


\begin{tabular}{l|l|} 
Farnacia \\
Clínica \\
Vol. 14 n.02 \\
Marzo 1997 \\
\hline
\end{tabular}

estabilidad a los cambios, como el DC de nuestro paciente. Más estudios son necesarios en la práctica clínica diaria para definir correctamente el patrón de cada paciente y su relación con los diferentes eventos clínicos.

El método de comparar los resultados individuales con poblaciones, ya sean pacientes o no, ha sido descrito con anterioridad en pacientes dializados y ha mostrado la validez de la utilización de cstos instrumentos en la práctica clínica diaria (19).

Los pacientes epilépticos tienen problemas emocionales que limitan su actividad social y funcional $(3,22,27)$. Aunque el SF-36 no mide directamente los problemas del sueño ni el miedo a las crisis (24), su utilización en nuestro paciente recoge la influencia de estos problemas en el curso de su tratamiento. La utilización de un cuestionario específico (por ejemplo: Seizure Severity Scale, Quality of Life in Epilepsy Inventory, The Liverpool Seizure Severity Scale) (21-23) además del genérico, permitiría una monitorización más estrecha de los pacientes epilépticos debido a que sería posiblemente más sensible a los cambios clínicos (20). Debido a que nuestro paciente ha sido intervenido quirúrgicamente, la administración del The UCLA Epilepsy Surgery Inventory (23) sería muy útil para realizar un seguimiento del mismo.

Como se detalla en la Tabla 1, el SF-36 puede ser utilizado para comparaciones individuales en pacientes epilépticos debido a que todas sus escalas presentan una alta consistencia interna, es decir, la fiabilidad de las mismas es superior a 0.7 (28). El tiempo medio utilizado para la administración de SF-36 en pacientes epilépticos fue de menos de 15 minutos, demostrando la comprensión del mismo por estos pacientes.

La alta calidad de los resultados obtenidos del SF-36 tanto en el número de respuestas como el índice de consistencia de las mismas es debida a la intervención del entrevistador tras la cumplimentación del cuestionario por el paciente (25). La calidad de los resultados obtenidos en la autoadministración del cuestionario es dependiente de la edad y del nivel sociocultural del entrevistado $(17,25)$

Nuestro paciente se encuentra por debajo de la norma en todas las escalas del SF-36 comparadas con una población adulta sin condición crónica preexistente a excepción de FF. En cambio Wagner y col. (3) mostraron que los pacientes epilépticos adultos tuvieron diferencias significativas en las escalas de PF y BP en

\section{Augmentine} AUGMENTINE INTRAVENOSO: Especialidad de uso hospitalario. COMPO-
SICION POR VIAL: 500/50: Amoxicilina (DCl) (sal sodica): $500 \mathrm{mg}$; ácido clavulánico (DC))(Sal potásica): $50 \mathrm{mg}$. 1g/200: Amoxicilina (DCl) (sal sód(a): $1.000 \mathrm{mg}$; ácido clavulänico (DCl) (sal potásica): $200 \mathrm{mg}, 2 \mathrm{~g} / 200$
Amoxicilina (DCI) (sal sódica): $2.000 \mathrm{mg}$ : ácido clavulánico (DC) (sal potásca): $200 \mathrm{mg}$ PROPIEDADES: Es un preprado antibacterinn de amplio espectro consifituído por amoxicilina (sal sódica) y ácido clavulánico (sa potásica). Ver detalle de 'gérmenes sensibles' en la literatura del producto. está indicatas. La asociacion de amoxiclina y acido clavulánico por via $i$. nes sensibles: respiratorias y OR.L (otitis medias, sinusitis, amiggalitisis. renales y uro-genitales (cistitis, uretritis, pielonefritis), ginecológicas (genitales), de piel y de teiddos blandos, intra-abdominales (en particular peritonitiss.

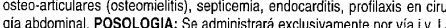
posología, por convención, se expresa en cantidad de amoxicilina. A) ADULTOS: Se utilizarán las presentaciones ' $\mathrm{g} / 200 \mathrm{mg}^{\prime}$ ' 2 ' $2 \mathrm{~g}^{\prime} 200 \mathrm{mg}$ '. Pacientes con función renal normal: La posologia habitual es de $1 \mathrm{~g}$, dos $0 \hat{c}$ cuatro

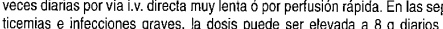
incluso hasta $12 \mathrm{~g}$ diarios. Jamás debe superarse, en un adulto, la cantided de $200 \mathrm{mg}$ de ácido clavulánico por administración y la de $1.200 \mathrm{mg}$ de ácido clavulanico al dia. Asi, para una dosis de hasia $6 \mathrm{~g}$ ciarios, se utilizará la pre

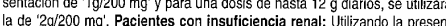
tación de '1g/200 mg' se dosificará con las cantidades siguientes, consider das como máximo: $1 \mathrm{~g}$ como dosis inicial $y$, a continuación, 500 o $250 \mathrm{mg}$ cada 12 horas en función del aclaramiento de creatinina, 10-30 $\mathrm{ml} / \mathrm{mn}$ ó 10 $\mathrm{m} / \mathrm{mnn}$ respectivamente. Profilaxis quirưrgica: $1 \mathrm{~g}$ durante la inducción a
anestesia en intervenciones de duración interior a 1 hora. En operaciones más duraderas, pueden ser necesarias más dosis de $1 \mathrm{~g}$ (hasta un máximo de $4 \mathrm{~g}$ en 24 horas). Si la intervención supone un allo riesgo de ińfección puede contifuarse esta administracioin durante varios dias, como terapia RECIEN NACIDOS: Se utilizará la presentación '500150 mg'. Niños y lactantes a partir de 3 meses $(5$ a $40 \mathrm{~kg}$ ): Usar $100 \mathrm{mg} / \mathrm{kg} / \mathrm{dia}$, en 4 admnistraciones al día por via i.y. directa muy lenta $\dot{o}$ por perfusión. En las Recién en 2 pertusiones al día. NORMAS PARA LA CORRECTA ADMINISTRACION: No preparar solución más que en el momento de la inyec-

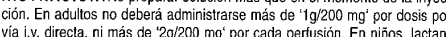
tes y recién nacidos no deberá administrarse más de $25 \mathrm{mg}$. $\mathrm{kg}$ por por dosis por via i.v. directa, ni más de $50 \mathrm{mg} / \mathrm{kg}$ por cada perfusión. La correcta adm. nistración del producto exige respetar unas normas estrictas sobre los disol ventes y volúmenes adecuados a utilizar, tiempo de administración, etc., por acompaña a cada presentación ó la intormación correspondiente en mono grafias y otros medios informalivos del producto. CONTRAINDICACIONES: Alergia a penicilinas, mononucleosis infecciosa, leucemia linfoide y asociaciún cont alupurinol. PRECAUCIONES: Debe administrarse con precaución un fondo alérgico, fundamentalmente medicamentoso. Usar con precaucion en los casos de insufficiencia renal, grave alteración hepática ó lactancia. Embarazo: No se ha establecido su inocuidad durante el mismo. INCOMgun otro producto en la mia genal se recomienda no mezclario con ninzarse como disolventes bicarbonato sódico ó dexitrano. Para más informaclón, consultar el prospecto incluido en cada presentación ó monografías y otros medios intormativos del producto. INTERACCIONES: Evitar su administración conjunta con antcentraciones altas, puede interferir con algunas determinaciones analificas. EFECTOS SECUNDARIOS: Trastornos de tipo digestivo \{náuseas, vómitos, etc.), alérgicos y erupciones cutáneas máculo-papulares. Más raramente, tis aguda citolitica ó colestática transitoria (con ó sin icteria), nefritits intersticial aguda, anemia, leucopenia y trombopenia reversibles y algunos casos de colitis pseudomembranosa tras la administración de amoxicilina. INTOXICACION Y SU TRATAMAENTO: Con las dosis recomendadas no se han dad, se suspenderá su administración y se aplicará el trat) inmento cifico (antihistamínicos, corticosteroides. adrenalina, etc.). PRESENTACIONES Y P.V.P. IVA-4: Frasco para perfusión '500/50 mg', 1 vial, 421 ptas., frasco; vial '4 g/200 mg', i vial, 734 ptas.; frasco para perfu$500 / 50 \mathrm{mg}$ EC 100 va $20.430 \mathrm{PU}$. $\mathrm{mg}$ EC 100 viales, 40.743 P.V.L. - 49.559 P.V.P. IVA. $2 \mathrm{~g} / 200 \mathrm{mg}$ EC 50 viales, 32.463 P.V.L.. - 39.487 P.V.P. IVA. Augmentine es marca registrada. Nombre y domicilio social del titular de S.A. Cl. Valle de la Fuenfría, 328034 MADRID. 
Resultados y discusión

24

relación a una población adulta sana equivalen te. Los resultados del paciente respecto a sus medias anteriores muestran que el patrón del dolor experimentado por el paciente no varía, lo que está en consonancia con la situación clínica del mismo y con la población de pacientes.

Al comparar los resultados de nuestro paciente con la norma de los pacientes epilépticos reflejan que su patrón difiere de esta población. Así por ejemplo, los resultados obtenidos en las escalas FF, SG y SM están casi siempre por encima de la norma, es decir estaría en el techo de esta población.

Las escalas del SF-36 con una validez substancial para valorar el estado funcional del paciente son FS, RF y DC $(17,25)$. Se observa en estas escalas al comparar con las normas de las poblaciones, qué número de pacientes tiene tan sólo problemas debidos a sus limitaciones físicas en dos momentos concretos, uno debido a la intervención quirúrgica y otro a un posible fracaso del tratamiento.

Aunque diferentes estudios demuestran que la CVRS varía al cabo de seis meses, la escala SG sólo detecta diferencias de la salud mental y física del paciente al cabo de un año entre ambas administraciones (25). Junto a SG y SM, la escala de VI es bipolar (24), y también mide ambos conceptos de la salud (física y mental) como SG, lo que significa que puntuaciones en la mitad de la misma no significan necesariamente una peor percepción de la salud general. Por lo que las pequeñas variaciones, como en el caso de nuestro paciente no pueden considerarse significativas ya que no varían del techo al suelo (25)

La medición de la CVRS sirve para evaluar los resultados de las intervenciones sanitarias $(17,23)$, lo cual se refleja en nuestro paciente ya que la hippocampectomía disminuye la frecuencia y la gravedad de las crisis epilépticas la cual se correlaciona con una mayor puntuación en las escalas RE. También el SF-36 recoge la disminución del estado funcional del paciente tras la misma en las escalas DC y FS. A diferencia de otro estudio (27) en la que la escala que mejor discriminó el grado de severidad de la enfermedad es el RF, en nuestro paciente fue también la escala RE.

\section{CONCLUSION}

El estado funcional y bienestar del paciente son medibles mediante la utilización de instrumentos válidos y fiables que miden la CVRS.
De esta manera podremos conocer la opinión del paciente sobre su tratamiento y enfermedad (13).

La medición de la CVRS en la práctica clínica de los pacientes epilépticos aporta nueva información a los clínicos que rutinariamente no se obtiene y puede servir de soporte en la toma de decisiones farmacoterapéuticas, además de ser un resultado en sí misma (13,17-19).

Cuantas más mediciones de la CVRS tengamos para valorar el tratamiento de un paciente epiléptico, con un instrumento genérico y específico (20), mejor se definirá el patrón del mismo y más posibilidades tendremos que la misma se ajuste a la realidad. Más estudios son necesarios para situar la información dada por los pacientes sobre su tratamiento en la práctica clínica.

\section{BIBLIOGRAFIA}

1. Garnet WR. Epilepsy. In: Pharmacotherapy. A pathophysiologic approach, 2nd Ed. Dipiro JT, Talbert RL, Hayes PE, et al. eds. Norwalk, Conneticut: Appleton \& Lange, 1993; 879-903.

2. Hausser WA, Hesdorffer DC. Facts about epilepsy. New York: Epilepsy Foundation of America, Demos Publications, 1990.

3. Wagner AK, Vickrey BG. The routine use of health-related quality of life measures in the care of patients with epilepsy: rationale and research agenda. Qual Life Res 1995; 4:169-77.

4. National Institutes of Health Consensus Development. Conference Statement. Surgery for epilepsy. Epilepsia 1990; 31:806-12.

5. Walker MC, Sander JWAS. The impact of new antiepileptic drugs on the prognosis of epilepsy: seizure freed be the ultimate goal. Neurology 1996; 6. Roper Organization. Living with epilepsy: report of a
Roper poll of patients on quality of life. New York: Author, 1992.

7. Leppik IE. Compliance in the treatment of epilepsy. In ed. The Treatment of Epileps Practice. Philadelphia: Lea and Febiger 1993; $810-$

8. Cramer JA, Scheyer RD, Mattson RH. Compliance declines between clinic visits. Ann Intern Med 1990; 150 1509-10.

9. Schor EL, Lerner DJ, Malspeis S. Physician's assessment of functional health status and well-being: The pa-
tient's perspective. Arch Intern Med 1995; 155:309-14.

10. Calkins DR, Rubestein LV, Cleary PD, et al. Failure of physicians to recognize functional disability in ambulaAnin Intern Med 1991; 114:451-4

11. Kozma CM, Reeder CE, Schulz RM. Economic, clinical, and humanistic outcomes: a planning model for pharmacoeconomic research. Clin Ther 1993; 15:112132 .

12. Ware JE. The status of health assessment 1994. Ann Rev Public Health 1995; 16: 327-54.

13. Testa MA, Simonson DC. Assessment of quality-of-life outcomes. N Engl J Med 1996: 332:835-40. 
Resultados y discusión

\begin{tabular}{|c|c|c|}
\hline $\begin{array}{l}\text { Farmacia } \\
\text { Clínica } \\
\text { Vol. } 14 \mathrm{n}^{\circ}{ }^{2} \\
\text { Marzo } 1997 \\
\end{array}$ & Original & 89 \\
\hline \multirow[t]{6}{*}{25} & $\begin{array}{l}\text { 14. Patrick DL, Erickson P. Health status and health policy. } \\
\text { Allocating resources to health care. New York: Oxford } \\
\text { University Press, } 1993 \text {. }\end{array}$ & $\begin{array}{l}\text { 24. Ware JE Jr, Sherbourne CD. The MOS 36-Item Short- } \\
\text { Form Health Survey (SF-36). Med Care 1992; 32:473- } \\
482 \text {. }\end{array}$ \\
\hline & $\begin{array}{l}\text { 15. Nelson ED, Landgraf, Hays RD, Wasson JH, Kirk JW. } \\
\text { The functional status of patients. How can it be measu- } \\
\text { red in physicians' offices?. Med Care 1990; 28:1111-26. }\end{array}$ & $\begin{array}{l}\text { 25. Ware JE, Snow KK, Kosinski M, Gandek B. SF-36 } \\
\text { Health Survery. Manual and Interpretation Guide. } \\
\text { Boston: The Health Institute, New England Medical } \\
\text { Center, } 1993 \text {. }\end{array}$ \\
\hline & $\begin{array}{l}\text { proving patient function: A randomized trial of functio- } \\
\text { nal disability screening. Ann Intern Med 1989; 111:836- }\end{array}$ & $\begin{array}{l}\text { 26. Response Technologies, Inc. The RT-2000 Immediate } \\
\text { Reponse Terminal. Operating Instruction. East } \\
\text { Greewich, RI: Respose Technologies, Inc., 1992. }\end{array}$ \\
\hline & $\begin{array}{l}\text { 17. Ware JE, Keller SD. Interpreting general health measu- } \\
\text { res. In: Quality of life and pharmacoeconomics in clini- } \\
\text { cal trials, 2nd Ed. Spilker B, ed.Philadelphia, PA: } \\
\text { Lippincott-Raven Publishers, 1996: } 445-60 \text {. }\end{array}$ & $\begin{array}{l}\text { 27. Wagner AK, Keller SD, Kosinski M, Baker GA, Jacoby } \\
\text { A, Hsu MA, Advances in methods for assessing the im- } \\
\text { pact of epilepsy and antiepileptic drugs therapy on pa- } \\
\text { tients' health-related quality of life. Qual Life Res 1995; } \\
\text { 4(2):115-34. }\end{array}$ \\
\hline & \multirow{2}{*}{$\begin{array}{l}\text { 18. Wagner AK, Bungay KM, Kosinski M, Bromfield EB, } \\
\text { Ehrenberg BL. The Health status of adults with epi- } \\
\text { lepsy compared with that of people without chronic } \\
\text { conditions. Pharmacotherapy 1996; 16:1-9. }\end{array}$} & $\begin{array}{l}\text { 28. Nunnally JC. Psycometric theory, 2nd ed. New York, } \\
\text { NY: McGraw-Hill, } 1978 .\end{array}$ \\
\hline & & bido: 11-11-96. \\
\hline
\end{tabular}

19. Meyer KB, Espindle DM, DeGiacomo JM, Jenuleson CS, Kurtin PS, Monitoring dialysis patients' health status. Am J Kidney Dis 1994; 24:267-79.

20. Aaronson NK. Methodologic issues in assessing the

21. Devinsky O, Vickrey BG, Cramer J, Perrine K, Hermann B, Meador K, Development of the quality of Hermann B, Meador K, Development of the quality o
life in epilepsy inventory. Epilepsia 1995; 36(11):1089104.

22. Hermann BP. The evolution of health-related quality of life assessment in epilepsy. Qual Life Res 1995; 4(2):87

23. Vickrey BG, Hays RD, Engel J Jr, Spritzer K, Rogers WH, Rausch R, Graber J, Brook RH. Outcome assessment for epilepsy surgery: the impact of measuring he-
alth-related quality of life. Ann Neurol 1995; 37(2):15866.

\section{AGRADECIMIENTOS}

Quisiera agradecer la confianza depositada en mi trabajo por el equipo editorial de Farmacia Clínica, quienes me sente trabajo.

Asimismo mi agradecimiento a los Dres. Anita K. Wagner (Health Institute), Ed Decker (Subdirector) y William A. Gouveia (Director) del Departamento de Farmacia del New te nuevo campo de investigción y por su enistad que tuve el honor de disfrutar durante mi estancia en su hospital. 


\section{Capítulo 5}

Carrera-Hueso FJ, Ramón Barrios A, Carrera Hueso JA, Poquet Jornet JE. Coste-efectividad de la tromboprofilaxis extendida en fractura de cadera: fondaparinux frente a enoxaparina. Aten Farm 2010; 12 (3): 144-55. 


\section{ORIGINAL}

CARRera Hueso FJ. FED. Farmacia Hospita-
laria.
Ramón Barrios A. TEL.
CARreRa Hueso JA. Jefe de Servicio de Anes-
tesia y Reanimación."
PoQuet Jornet JE. Dr. en Farmacia. Jefe de
Área Clínica de Farmacia."
Servicio de Farmacia. Hospital Dr. Moliner.
Serra (Valencia).
"Hospital Francisco de Borja. Gandía (Va-
lencia).
"Hospital de Denia (Alicante).
Aten Farm 2010; 12(3): 144-55.
Recibido: 23-10-09. Aceptado: 3-2-10.

Recibido: 23-10-09. Aceptado: 3-2-10.

\section{COSTE-EFECTIVIDAD DE LA TROMBO- PROFILAXIS EXTENDIDA EN FRACTURA DE CADERA: FONDAPARINUX FRENTE A ENOXAPARINA}

Carrera Hueso Francisco Javier, Ramón Barrios Auxiliadora, Carrera Hueso José Adolfo, Poquet Jornet Jaime Eduardo

\section{RESUMEN}

Se compara la relación coste-efectividad de fondaparinux $2,5 \mathrm{mg}$ diarios administrados subcutáneamente durante cuatro semanas frente a enoxaparina $40 \mathrm{mg}$ diarios durante seis semanas subcutáneos, para la prevención de la enfermedad tromboembólica venosa en pacientes sometidos a cirugía por fractura de cadera.

Se ha seleccionado un modelo de análisis de decisión simplificado con un horizonte temporal de cinco años y tasa de descuento del $5 \%$. El análisis de sensibilidad (univariado, bivariado, trivariado) se ha realizado para las variables, tanto costes como eventos tromboembólicos, que pueden determinar la selección de las alternativas.

El coste promedio obtenido por paciente fue de 1.507 y $583 €$ para enoxaparina y fondaparinux, respectivamente. Sin embargo, el ratio coste-efectividad para enoxaparina y para fondaparinux fue de 15,31 y de $1881,61 €$ por evento tromboembólico sintomático, respectivamente. El análisis de sensibilidad confirma la selección de fondaparinux como alternativa tromboprofiláctica más costeefectiva que enoxaparina.

Concluimos que las pautas posológicas evaluadas y los datos y asunciones del modelo utilizado, el tratamiento de la tromboprofilaxis extendida de la enfermedad tromboembólica venosa tras fractura de cadera con fondaparinux evidencia una relación coste-efectividad superior a enoxaparina.

Tromboprofilaxis - FondAPARINUX - EnOXAPARINA - Fractura de CADERA COSTE-EFECTIVIDAD

\section{ABSTRACT}

We compared the cost-effectiveness relationship of $2.5 \mathrm{mg}$ fondaparinux daily administered subcutaneously for four weeks versus $40 \mathrm{mg}$ enoxaparin daily administered subcutaneously for six weeks, for preventing the venous thromboembolic disease in patients undergoing hip fracture surgery.

We have chosen a simplified decision analysis model with a 5-year timeframe and a 5\% discount rate. The sensitivity analysis (univariate, bivariate and trivariate) has been performed for the variables, both cost and thromboembolic events, that could determine the choice of alternatives.

The average cost per patient was $€ 1,507$ for enoxaparin and $€ 583$ for fondaparinux. Nonetheless, the cost-efficiency ratio for enoxaparin was $€ 15.31$ and for fondaparinux $€ 1,881.61$ per symptomatic thromboembolic event. The sensitivity analysis confirmed the choice of fondaparinux as a more cost-effective thromboprophylactic alternative than enoxaparin.

We conclude that the dosage patterns, data and assumptions in the model show a higher cost-efficiency relationship for fondaparinux than enoxaparin in the treatment of extended thromboprophylaxis for the venous thromboembolic disease after hip fracture surgery.

THROMBOPROPHYLAXIS - FONDAPARINUX - ENOXAPARIN - HIP FRACTURE - COST-EFFECTIVENESS 
Resultados y discusión

\section{INTRODUCCIÓN}

Tanto la trombosis venosa profunda (TVP) como el embolismo pulmonar (TEP) son considerados una misma entidad patológica, la enfermedad tromboembólica venosa (ETV). La incidencia global de la TVP en España es de cinco casos por 10.000 habitantes/año, menor que en Francia o en Suecia donde asciende hasta 18 casos por 10.000 habitantes/año y, superior a China (dos casos por 10.000 habitantes/año). ${ }^{1,2}$

La ETV continúa siendo un problema sanitario a largo plazo aunque se realice una profilaxis correcta y exhaustiva de todos los pacientes sometidos a cirugía ortopédica mayor (COM). Además de la propia COM como importante factor de riesgo, existen varios factores de riesgo relacionados con ETV: primarios, como trombofilias o, secundarios, como la edad, el tipo de cirugía, tipo de técnica anestésica, cáncer, obesidad, embarazo e infarto agudo de miocardio, entre otros. ${ }^{1,3}$

Son frecuentes sus consecuencias a largo plazo: la TVP recurrente y el síndrome postflebítico (SPT), con secuelas de dolor, edema, mala perfusión cutánea, hiperpigmentación, ulceraciones. ${ }^{3}$ Un estudio prospectivo de la evolución a largo plazo de 528 pacientes con TVP sintomática observó que a los cinco años una cuarta parte de los enfermos que sufrieron una TVP, desarrollaron una trombosis recurrente y casi un tercio presentaban síntomas de SPT. 4

En pacientes que reciben una tromboprofilaxis de duración adecuada se produce un retardo en la aparición de complicaciones tromboembólicas, apareciendo un segundo pico de incidencia tras las tres semanas de la intervención. La introducción de la profilaxis ha representado una modificación importante de la historia natural del TEP después de COM, con un descenso significativo de la prevalencia de TVP en el postoperatorio inmediato. ${ }^{1,5}$

La prevención primaria de ETV después de COM incluye métodos mecánicos y farmacológicos. Los mecánicos son las medias elásticas, la compresión neumática intermitente y la bomba venosa plantar, que han demostrado su eficacia, solas o en combinación con medicamentos. La profilaxis farmacológica se realiza con heparina no fraccionada (HNF) a dosis bajas o ajustadas, anticoagulantes orales tipo warfarina, heparinas de bajo peso molecular (HBPM), inhibidores directos de la trombina (hirudina) o inhibidores específicos del factor $\mathrm{Xa}$ como el fondaparinux 3,5

Las HBPM han sido evaluadas económicamente frente a otras alternativas farmacológicas en la tromboprofilaxis tras COM. Entre ellas cabe destacar las realizadas frente a warfarina, siendo más coste-efectivas las HBPM que la warfarina. ${ }^{6-17}$ La ventaja de las HBPM es debida a que no se necesitan controles continuos para comprobar su eficacia, como ocurre con warfarina. Otros estudios económicos en los que se comparan las HBPM frente a diversos fármacos, como desuridina ${ }^{18} \mathrm{o}$ danaparoide, ${ }^{11,19,20}$ también se ha puesto de manifiesto la superioridad de las HBPM, así como en situaciones traumatológicas de urgencias frente a $\mathrm{HNF}^{21}$
Numerosas publicaciones sustentan la utilización de la tromboprofilaxis prolongada frente a la de duración normal dado que se presentan menores eventos trombóticos a corto y a largo plazo. Entre los fármacos utilizados para el tratamiento de la ETV destaca la enoxaparina, HBPM y fármaco de referencia que ha desplazado a la warfarina en esta indicación. ${ }^{17,22-40}$

Tras la incorporación de fondaparinux, pentasacárido sintético que ha demostrado ser más eficiente en la tromboprofilaxis de la ETV tras COM que enoxaparina aunque con un mayor coste de adquisición, es necesario situarlo dentro del arsenal terapéutico disponible. ${ }^{41} \mathrm{E}$ fondaparinux ha sido también centro de estudios económicos comparándolo con HBPM. Los resultados obtenidos con tromboprofilaxis estándar, son contradictorio ya que algunos dan dominante a la enoxaparina ${ }^{42,43}$ y otros al pentasacárido, sobre todo los estudios que tenían en cuenta las consecuencias a largo plazo, desde 90 días hasta cinco años. ${ }^{44-51}$

Con la tromboprofilaxis prolongada, esto es seis semanas para las HBPM o cuatro semanas para fondaparinux, se ha conseguido disminuir el número de eventos tromboembólicos sin aumento de los efectos secundarios en la profilaxis de COM. Los estudios económicos comparan la profilaxis extendida de las HBPM frente a la tradicional, demostrando una mejor relación coste efectividad para la: estrategia prolongada con enoxaparina ${ }^{52-55}$ o dalteparina. ${ }^{17}$ También fondaparinux ha demostrado mejores resultados farmacoeconómicos con la estrategia profiláctica de larga duración. ${ }^{56}$

Se ha comparado enoxaparina frente a warfarina en profilaxis entendida durante 21 días tras el alta hospitalaria ${ }^{57} \mathrm{y}$ anteriormente se evalúo la HBPM con la HNF durante cuatro semanas tras intervención de prótesis total de rodilla; ${ }^{23}$ siendo la estrategia más coste efectiva las HBPM en todos estos estudios.

No se han realizado estudios farmacoeconómicos en nuestro país que contemplen las consecuencias a largo plazo de la ETV, como el síndrome postrombótico y la TVP recurrente, cuando se ha utilizado la tromboprofilaxis extendida, es decir, más allá de los días de hospitalización, que comparen fondaparinux frente a HBPM Tan sólo existe constancia de un trabajo internacional realizado comparando el pentasacárido con enoxaparina en tromboprofilaxis extendida en pacientes intervenidos tras fractura de cadera. ${ }^{58}$

El objetivo principal del presente estudio es determinar qué fármaco, fondaparinux o enoxaparina, a las pautas posológicas habituales, presenta mejor relación coste-efectividad para la prevención de la ETV en pacientes intervenidos tras fractura de cadera. Como objetivos secundarios del estudio podemos enumerar: conocer la distribución del coste medio por paciente de cada alternativa farmacológica evaluada; evaluar cómo las diferentes probabilidades de los eventos ETV, tanto las TVP sintomáticas o asintomáticas y el TEP afectan a la selección más coste-efectiva de la tromboprofilaxis; y comprobar cuanto varía y qué dirección las posibles diferencias entre las estrategias de tromboprofilaxis pro- 
Resultados y discusión

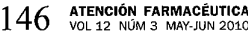

Carrera Hueso FJ, Ramón Barrios A, Carrera Hueso JA, Poquet Jornet JE

tabla 1. Probabilidades de los sucesos.

\begin{tabular}{|c|c|c|c|}
\hline Sucesos & Enoxaparina & Fondaparinux & Fuente \\
\hline \multicolumn{4}{|l|}{ Primera semana (días 1-7) } \\
\hline Eventos trombóticos sintomáticos & 0,0156 & 0,0068 & $41,58,61$ \\
\hline Hemorragias mayores & 0,0160 & 0,0260 & 41,61 \\
\hline \multicolumn{4}{|l|}{ Primer mes (días 8-30) } \\
\hline TVP asintomáticas & 0,2210 & 0,0114 & $27,44,61$ \\
\hline Eventos trombóticos sintomáticos & 0,0149 & 0,0031 & 27,61 \\
\hline \multicolumn{4}{|l|}{ Tres meses (días 31-90) } \\
\hline Eventos trombóticos sintomáticos & 0,0388 & 0,0388 & 25,61 \\
\hline TVP recurrente & 0,0397 & 0,0397 & 1,25 \\
\hline \multicolumn{4}{|l|}{ Hasta 5 años } \\
\hline TVP recurrente & 0,0397 & 0,0397 & ' \\
\hline Síndrome postrombótico & 0,1730 & 0,1730 & 4 \\
\hline Embolismo pulmonar & 0,0010 & 0,0012 & 61,62 \\
\hline
\end{tabular}

longada frente a las estrategias profilácticas restrictivas en pacientes intervenidos por fractura de cadera.

\section{MÉTODO}

Alternativas y perspectiva. Los tratamientos que se compararon fueron $2,5 \mathrm{mg}$ de fondaparinux vía s.c. una vez al día durante cuatro semanas iniciándose en las 4-8 primeras horas del postoperatorio inmediato, y $40 \mathrm{mg}$ de enoxaparina diaria mediante inyección subcutánea, iniciándose el tratamiento 12 horas antes de la intervención quirúrgica y prolongándose hasta seis semanas después. Ello supone 28 unidades de fondaparinux (Arixtra ${ }^{\circledR}$, $2,5 \mathrm{mg}$ ) y 43 inyecciones precargadas de enoxaparina (Clexane ${ }^{\circledR}, 40 \mathrm{mg}$ ) por paciente tratado. No se contempla en el modelo la primera dosis de enoxaparina al tomar como referencia de costes y eficacia, estudios de enoxaparina que no la tuvieron en cuenta. $26,27,53,59$

Al igual que el resto de trabajos realizados en este campo el horizonte temporal fue de cinco años para as contemplar las consecuencias a largo plazo de la ETV producida tras COM..$^{37,44,49,58,59}$

La perspectiva tomada fue la del proveedor o financiador público de los servicios sanitarios, que es el predominante en nuestro sistema sanitario y también en el resto de la Unión Europea.

Descripción del modelo utilizado y asunciones. Se ha utilizado un modelo ${ }^{44,60}$ simplificado de análisis de decisión mostrado por Slof y Badía,${ }^{59}$ que representa la evolución natural de la ETV y su resolución con los tratamientos comparados a largo plazo.

Se asume que los pacientes que sufren una TVP, bien sea en el ámbito hospitalario o ambulatorio, presente o no síntomas, sufrirán en el futuro secuelas expresadas bien como síndrome postrombótico, TEP o TVP recurrente. Los episodios pueden suceder de manera aguda, en el hospital, o al alta hospitalaria y hasta los cinco años. Transcurrido ese tiempo no se puede imputar un evento nuevo ni al procedimiento quirúrgico ni a la profilaxis realizada. De ahí que para los eventos considerados tardíos en el modelo, sus probabilidades se consideren prácticamente idénticas excepto para el SPT. Los eventos considerados hasta cinco años son, además del SPT, las TVP recurrentes $y$ el TEP, o que no existan complicaciones o secuelas.

Aunque no se contemple la mortalidad en el modelo simplificado, sí se ha considerado el riesgo de hemorragias mayores debidas o imputadas al riesgo profiláctico. Es un aspecto relevante al ser el principal efecto secundario de ambas estrategias profilácticas y que pueden no sólo comprometer su eficacia sino incluso la propia salud de los pacientes.

Para su desarrollo se ha utilizado el programa informático Microsoft Office Excell 2003, Data 3.5 (Tree Age Software 1998) y Elvira versión 0.162.

Determinación de las probabilidades de los episodios. La efectividad de los tratamientos se ha obtenido a partir de ensayos clínicos a doble ciego que evaluaban la profilaxis extendida de cada fármaco comparada con su propia estrategia profiláctica restrictiva, controlándose con placebo este grupo durante la prolongación de la tromboprofilaxis. Los principales estudios utilizados han sido los que han usado profilaxis extendida de cuatro y seis semanas para fondaparinux y enoxaparina respectivamente, en pacientes intervenidos de fractura de cadera ${ }^{61}$ o de prótesis de rodilla y de cadera. ${ }^{25} \mathrm{En}$ ambos casos, el objetivo principal del estudio fue la medida de la eficacia de los tratamientos profilácticos expresada como la prevalencia de ETV (número de episodios de TVP y TEP confirmadas). La principal međida de seguridad fueron las hemorragias mayores acontecidas.

Se han utilizado los datos tanto de efectividad como de reacciones adversas de la evidencias disponibles de la profilaxis con enoxaparina y de fondaparinux durante seis y cuatro semanas respectivamente, para el cálcu- 
Resultados y discusión

$148 \begin{gathered}\text { ATENCIÓN FARMACÉUTTCA } \\ \text { VOL } 12 \text { NUM } 3 \text { MAA-UUN } 2010\end{gathered}$

Carrera Hueso FJ, Ramón Barrios A, Carrera Hueso JA, Poquet Jornet JE

tabla 2. Costes en euros (año 2008).

\begin{tabular}{ll}
\hline Eventos & Costes ( $\epsilon$ ) \\
\hline Profilaxis: & \\
- Enoxaparina (43 dosis de $40 \mathrm{mg}$ ) & 220,50 \\
- Fondaparinux (28 dosis de $2,5 \mathrm{mg}$ ) & $386,68$. \\
TVP detectada durante la estancia hospitalaria & $4.172,71$ \\
TVP detectada clínicamente después del alta hospitalaria & $1.646,10$ \\
TVP sintomática tras el alta hospitalaria, actualizada a los 2 años & $3.784,77$ \\
TEP detectada clínicamente después del alta hospitalaria a los 5 años & $4.240,72$ \\
TVP asintomática (sospecha de TVP y EP) a los 2 años. & 675,96 \\
Hemorragias graves relacionadas con la profilaxis & $3.075,27$ \\
Síndrome postrombótico (fase aguda un trimestre y fase crónica & \\
$\quad$ el resto de los trimestres) a los 5 años & 819,08 \\
TVP recurrente a los 5 años & $1.289,76$ \\
\hline TVP: Trombosis venosa profunda. TEP: Embolismo pulmonar. EP: Embolismo pulmonar. & \\
\hline
\end{tabular}

lo de las probabilidades condicionadas del modelo, muchas de las cuales se obtienen directamente de los ensayos clínicos controlados. ${ }^{25,61}$ El resto de probabilidades calculadas se han extraído de la bibliografía disponible y concretamente de evaluaciones económicas previas realizadas.

Las TVP asíntomáticas o flebográficas se deben incluir en el modelo, a pesar de que no se realice la técnica rutinariamente en la práctica clínica diaria, porque se ha demostrado que causan igualmente secuelas e incluso la muerte por TEP masivo.

Aunque no se encuentra descrito en los trabajos consultados como tal, 25, 58, 59,61 en las TVP experimentadas tras el alta hospitalaria se incluyen, por congruencia del modelo, tanto las asintomáticas como las sintomáticas producidas con posterioridad.

Análisis de costes. Los costes tenidos en cuenta son los soportados por el Sistema Nacional de la Salud, esto es atención médica, medicamentos, hospitalizaciones, etc.; excluyéndose otro tipo de servicios socio-sanitarios que necesitasen los pacientes. Por lo que estos costes son los sanitarios directos, en euros del año 2008. No se contemplan ni los costes indirectos derivados de la incapacidad laboral de los pacientes, ni los costes intangibles (dolor producido, etc.), ni los sanitarios no directos.

Los precios de venta al público de los fármacos utilizados en tromboprofilaxis son los que se han tenido en consideración según el Catálogo del Consejo General de Colegios Oficiales de Farmacéuticos 2008.

El resto de costes se han extraído de la base de datos SOIKOS, utilizando los costes de los principales Grupos Relacionados con el Diagnóstico (GRD) implicados: GRD 128: tromboflebitis de las venas profundas; GRD 78: embolia pulmonar; GRD 174: hemorragias digestivas con complicaciones; GRD 175: hemorragias digestivas sin complicaciones. Los costes han sido actualizados desde el año 2005 al año 2008 , a partir de los datos extraídos del trabajo de Slof y Badía, ${ }^{59}$ ya que esta base de datos no se encuentra disponible en la actualidad.

TABLA 3. Coste medio por paciente en euros (año 2008).

\begin{tabular}{lll} 
Evento & Enoxaparina & Fondaparinux \\
\hline Profilaxis & 220,50 & 386,68 \\
Hemorragias & 24,11 & 15,17 \\
TVP hospital & 23,51 & 3,66 \\
TVP sintomática hospital & 9,04 & 22,17 \\
TVP tras alta & 355,54 & 8,46 \\
TVP sintomática tras alta & 461,48 & 9,16 \\
TVP asintomática & 77,32 & 6,65 \\
TEP & 15,07 & 7,00 \\
SPT & 260,74 & 100,91 \\
TVP recurrente & 59,83 & 23,16 \\
Total & 1507,15 & 583,01 \\
\hline TVP: Trombosis venosa profunda. TEP: Embolismo pulmonar. SPT: Síndrome post-trombótico. \\
\hline
\end{tabular}




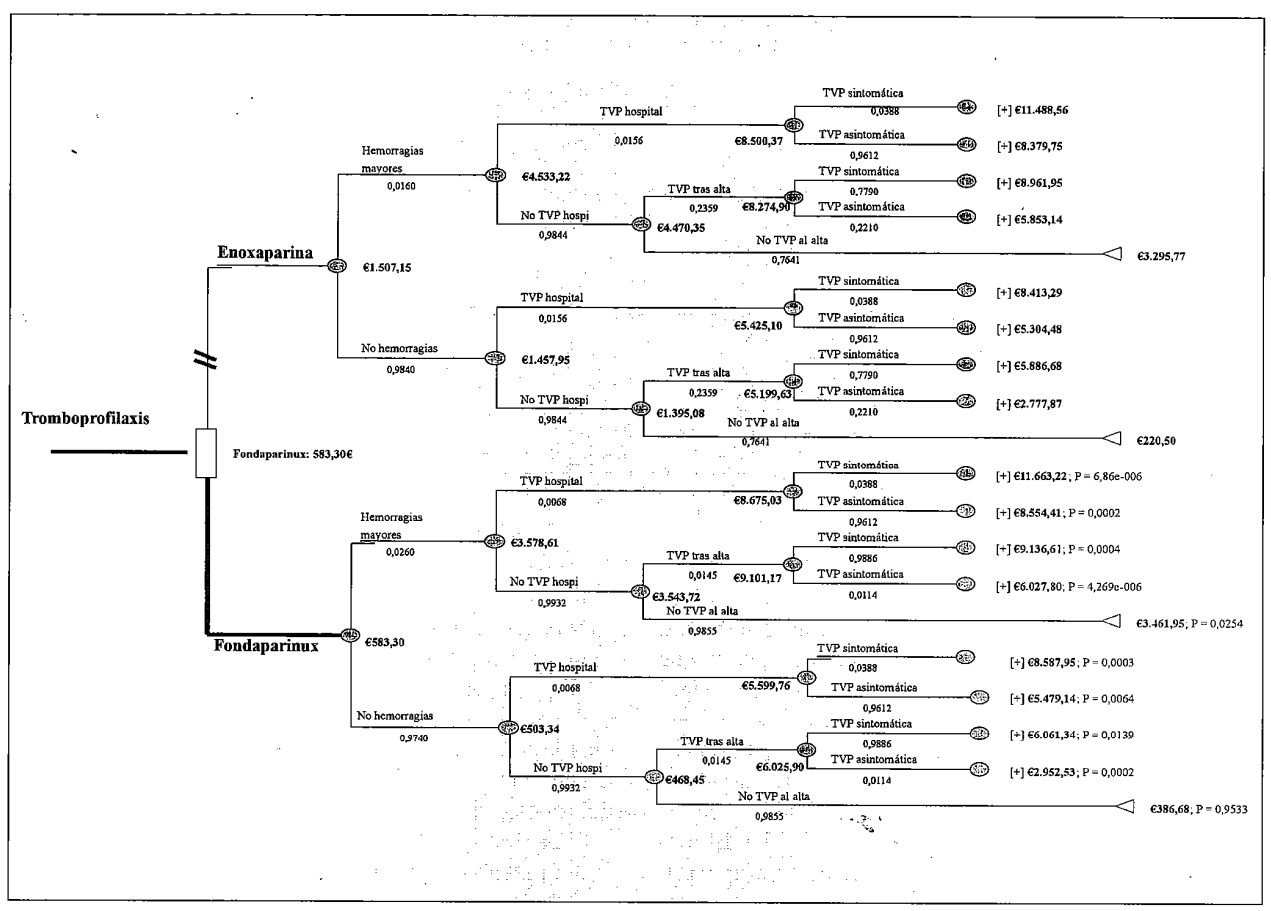

FIG. 1. Resolución del modelo (costes).

La tasa de descuento utilizada es del $5 \%$ debido a que las condiciones de la coyuntura económica prevista en 2008. Todos los indicadores que marcan esta tendencia estuvieron en torno a esta cifra durante el año 2007, como el euribor y el IPC.

Algunos costes, concretamente de las TVP sintomáticas y asintómáticas, se han descontado a dos años (con la tasa de descuento del $5 \%$ ) ya que se ha considerado que estos eventos pueden aparecer en este espacio de tiempo y no posteriormente según la evolución natural de la enfermedad. Otros costes como el SPT o las TVP recurrentes, se han imputado directamente a los cinco años, debido a su aparición tardía o cronicidad. El TEP también se hà tomado a cinco años ya que el modelo simplificado ${ }^{59}$ así lo requería a pesar de no ser únicamente de aparición tardía.

Para estudiar el coste promedio de cada paciente en cada una de las estrategias profilácticas utilizadas, se simuló una cohorte de 10.000 pacientes para cada una de las alternativas, obteniéndose el número de pacientes asignados en cada rama o nodo final del árbol de decisión. El coste total de cada rama del árbol o resultado se obtuvo sumando los diversos costes acarreados, multiplicándolos posteriormente por el número de pacientes de ese resultado.
Análisis de coste-efectividad. Para poder valorar la relación coste-efectividad de los tratamientos, se calculó el ratio coste-efectividad incremental (RCEI) de una alternativa frente a la otra que se compara. El cálculo se realiza de la siguiente manera, (tomando A y B como alternativas):

$$
\mathrm{RCEI}=\Delta \mathrm{C} / \Delta \mathrm{E}=\mathrm{C}_{\mathrm{A}}-\mathrm{C}_{\mathrm{B}} / \mathrm{E}_{\mathrm{A}}-\mathrm{E}_{\mathrm{B}}
$$

Siendo: $C_{A}$ : costes de la alternativa $A ; C_{B}$ : costes de la alternativa $B ; E_{A}$ : eficacia o efectividad de la alternativa $A ; E_{B}$ : eficacia o efectividad de la alternativa $B$.

Nótese la diferencia de calcular los ratios promedios de cada alternativa y compararlos, es decir calcular $\mathrm{C}_{\mathrm{A}} / \mathrm{E}_{\mathrm{A}}$ y $\mathrm{C}_{\mathrm{B}} / \mathrm{E}_{\mathrm{B}}$ y compararlos, a diferencia del RCEI que nos informa sobre lo que costaría (en dinero) conseguir una unidad más de eficacia.

Análisis de sensibilidad. En los resultados obtenidos con el modelo pueden influir varios factores que incorporan incertidumbre. La incidencia de los episodios o eventos se basa en probabilidades derivadas de estudios empíricos expuestos a variación aleatoria. También se debe tener presente que los datos de los ensayos clínicos controlados han sido obtenidos en condiciones ideales que no 
Resultados y discusión

TABLA 4. Análisis de sensibilidad univariado: coste medio por paciente en $€$.

\begin{tabular}{|c|c|c|c|}
\hline Parámetro modificado & Enoxaparina & Fondaparinux & Decisión ${ }^{*}$ \\
\hline Tasa de descuento $3 \%$ & $1.552,24$ & 581,72 & Fondaparinux \\
\hline Tasa de descuento $7 \%$ & $1.507,15$ & 614,44 & Fondaparinux \\
\hline Sin realizar descuentos, resultados en 1 año & $1.598,27$ & 590,82 & Fondaparinux \\
\hline Coste de TVP asintomática $=0 €$ & $1.462,32$ & 578,77 & Fondaparinux \\
\hline $\begin{array}{l}\text { Coste de TVP sintomática hospitalaria } \\
\text { de } 1.207,35 €\end{array}$ & $1.460,89$ & 563,13 & Fondaparinux \\
\hline Hemorragias por fondaparinux $>4 \%$ & $1.507,15$ & 632,5 & Fondaparinux \\
\hline $\begin{array}{l}\text { Menor efectividad de fondaparinux } \\
(\% \text { ETV sintomática }=4,2)\end{array}$ & $1.507,15$ & 587,99 & Fondaparinux \\
\hline Efectividad (\% ETV sintomática) $)^{25,61}$ & 1,49 & 0,31 & Fondaparinux \\
\hline
\end{tabular}

representan necesariamente la práctica clínica habitual. En cuanto al consumo de recursos sanitarios, se puede haber inducido a errores de estimación, tanto en cuanto a su cantidad como en cuanto a su coste unitario. Por estas razones, se procedió a un análisis de sensibilidad haciendo variar ampliamente los parámetros fundamentales del modelo alrededor de la situación base.

Se efectuó un análisis unifactorial con el fin de contrastar la robustez de los resultados teniendo que variar las asunciones realizadas, como la tasa de descuento, evaluando el rango del 3 al $7 \%$. Otras variables modificadas de manera univariada fueron:

- Utilizar los costes en el año corriente sin descontar - Asignar coste cero a TVP asintomática, ya que en la realidad no es un coste que se pueda imputar al no realizarse pruebas diagnósticas por pasar desapercibida para los pacientes.

- Asignar 1.207,35 € como coste de la TVP sintomática hospitalaria puesto que es el coste asignado en el estudio ya publicado, ${ }^{58}$ actualizado al año 2008.

- Asumir que el número alto de hemorragias mayores imputables al fondaparinux $(>4 \%)$.

- Asumir menor efectividad de fondaparinux, es decir aumentando la TVP sintomática al 4,2\%.

Debido a que a veces pueden condicionar la decisión al modificar distintas variables conjuntamente, se realizó también un análisis multivariado. Este análisis fue bivariado y trivariado, consistente en modificar no sólo uno, sino varios parámetros a la vez.

\section{RESULTADOS}

Para cada estrategia terapéutica, fondaparinux o enoxaparina, se recogen en la Tabla 1, las probabilidades condicionales de cada suceso así como las fuentes utilizadas. Por tanto, el porcentaje de pacientes con una ETV sintomática tras tromboprofilaxis con $40 \mathrm{mg}$ sc de enoxaparina durante seis semanas fue del 1,49 y del $0,31 \%$ para el grupo tratado con $2,5 \mathrm{mg}$ sc de fondaparinux durante cuatro semanas.

En la Tabla 2 se muestran los costes de cada evento del análisis de decisión utilizado. Se puede observar un mayor coste de $166,18 €$ que supone una estrategia profiláctica frente a la otra, debido al coste de adquisición del fondaparinux.

Los costes promedio por paciente fueron de $1.507 \mathrm{y}$ $583 €$ para enoxaparina y fondaparinux, respectivamente. Estos costes aparecen desglosados en la Tabla 3 para cada uno de los fármacos utilizados. Sin embargo, el ratio coste efectividad para enoxaparina y para fondaparinux fue de 15,31 y de $1.881,61 €$, por evento tromboembólico sintomático, respectivamente. Dicho de otra manera, el coste tromboembólico sintomático evitado fue 15,3 y $5,85 €$ para enoxaparina y fondaparinux, respectivamente.

En la Fig. 1 se muestra el modelo resuelto gráficamente con el programa DATA 3.5 cuando los resultados finales son los costes de cada rama, desarrollando el árbol de decisión para la alternativa menos costosa.

Del análisis coste incremental se deduce una dominancia absoluta del fondåparinux frente a la enoxaparina ( $\mathrm{RCEI}=-782,93 €$ por evento evitado), al presentar una mayor efectividad y un menor coste total el pentasacárido.

Los resultados de los parámetros evaluados en el análisis de sensibilidad univariado se muestran en la Tabla 4. A pesar de someter a variación tanto distintas situaciones económicas como las diferentes tasas de descuento utilizadas ( 3 y $7 \%$ ), así como la utilización de un horizonte temporal más corto, o una menor efectividad del fondaparinux o mayor toxicidad del mismo, los resultados obtenidos no cambian, siendo siempre fondaparinux la mejor estrategia profiláctica.

Al realizar el análisis de sensibilidad univariado con la probabilidad de hemorragias mayores con fondaparinux puede conocerse que sólamente cambiaría la decisión final cuando la misma sea mayor al 33\%, su- 
Resultados y discusión

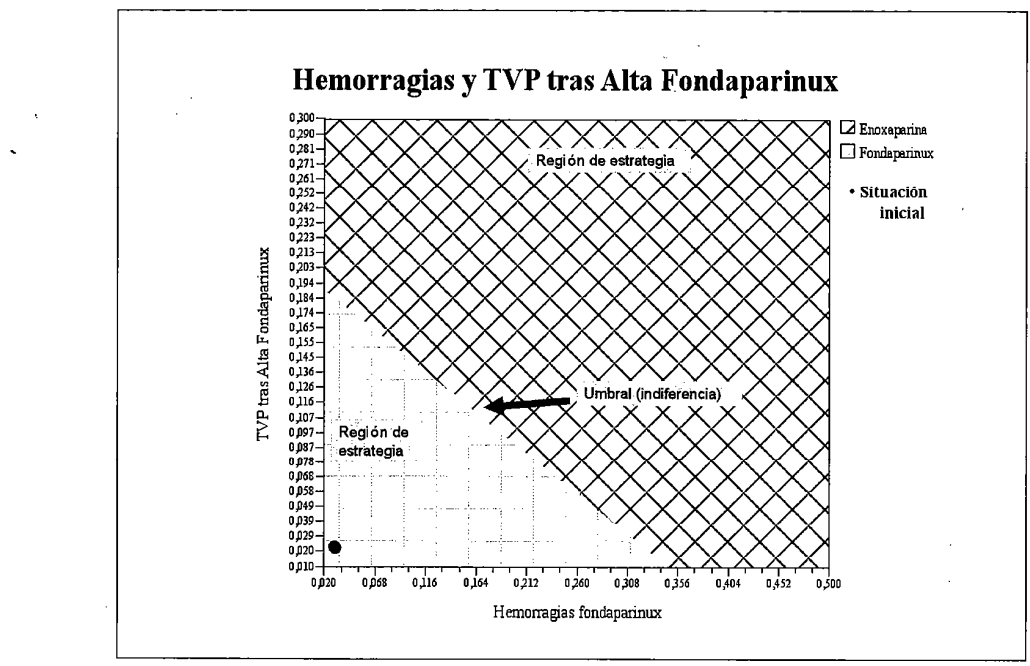

FIG. 2. Análisis de sensibilidad bivariante.

puesto lógicamente muy lejos de la realidad, concretamente del $2,4 \%$ comunicado en el estudio pivotal del fármaco. ${ }^{61}$

En la Fig. 2 se presentan los resultados de un análisis de sensibilidad bivariante. Como se observa, en la mayor parte de las combinaciones la elección de la profilaxis nó cambia respecto al caso base, es decir fondaparinux es dominante.

La interpretación del análisis de sensibilidad bivariante es la siguiente: la línea umbral o la que denota indiferencia entre las alternativas es la que separa ambas zonas cuadriculadas y el punto es el caso base o de partida. En base a estos resultados, las probabilidades de TVP tras el alta y las hemorragias de fondaparinux se deberían variar mucho conjuntamente desde el estado inicial para que la decisión cambiase de zona de decisión como se muestra en la Fig. 2. Este hecho concuerda con el análisis univarido de cada una de las variables consideradas por separado.

Por otra parte, la interpretación del análisis de sensibilidad trivariante no es tan directa ni sencilla pero se puede intuir una interpretación similar, teniendo presente la variable no representada gráficamente, en nuestro caso la probabilidad de trombosis venosa profunda durante la estancia hospitalaria.

\section{DISCUSIÓN}

Los resultados obtenidos con el presente modelo, a largo plazo (5 años de seguimiento), muestran que la alternativa más coste-efectiva fue fondaparinux, es decir, que fue más efectiva y con un menor coste total para los pacientes. Estos resultados coinciden con los publicados por Lundkvist ${ }^{58}$
Como puede observarse en la Tabla 3, el menor coste por paciente del grupo de fondaparinux se obtuvo por un menor coste en el tratamiento de los episodios tromboembólicos, tanto los inmediatos como los tardíos (SPT, TEP tardío o TVP recurrente). El mayor número de hemorragias mayores y del coste de adquisición del fármaco no compensa este ahorro obtenido de $924 €$ respecto a la enoxaparina, medicamento de referencia en nuestro medio. En el otro estudio ${ }^{58}$ obtuvieron los mismos resultados pero la diferencia entre ambos tratamientos profilácticos fue menor, de 52,5€, aunque la distribución de costes proporcionalmente fue muy similar a los obtenidos en nuestro trabajo para ambos grupos de pacientes tratados.

Estas discrepancias en costes con el estudio sueco ${ }^{58}$ pueden ser debidos a que en este trabajo se ha utilizado un modelo simplificado y"se ha utilizado un coste de referencia diferente tanto para el tratamiento de un episodio de TVP en hospitales y como para tratar las hemorragias mayores. En ese estudio se tomaron valores de $1.095,1 €$ y $1.483,3 €$ (euros año 2006) respectivamente, mientras que en nuestro trabajo se ha tomado un coste de 4.172,71 € y de 3.075,27€ (euros año 2008), similar al seleccionado por otros autores. ${ }^{43}, 59$ Las diferencias también pueden ser atribuidas a la distinta situación geográfica o a la toma de diferentes años del valor de la moneda. En nuestro caso, es poco plausible que sean debidas a estas razones ya que sería como asegurar que la asistencia sanitaria en Suecia es mucho más barata que en España. Sin embargo, en ambos trabajos las probabilidades de los eventos han sido muy parecidas al tomar como referencia el estudio PENTHIFRA Plus. ${ }^{61}$

Otra posible explicación de esta amplia diferencia 
Resultados y discusión

es que el tamaño del efecto de la tromboprofilaxis es mayor en los pacientes intervenidos de fractura de cadera que en los sometidos al resto de COM (artroplastia total de rodilla o cadera). ${ }^{41,62-64}$ Así, se ha manifestado también en las evaluaciones económicas de tromboprofilaxis a corto plazo en diferentes escenarios geográficos y temporales. ${ }^{45-47,50}$

Los resultados obtenidos en el análisis coste-efectividad de la profilaxis prolongada respecto a la evidencia disponible en evaluaciones económicas, comparando ambos fármacos en profilaxis estándar, muestran algunas peculiaridades que se confirman en el presente estudio. Por ejemplo, realizar un seguimiento a largo plazo de las consecuencias, favorecen a la opción profiláctica con fondaparinux independientemente del tiempo de administración de los fármacos, como se pone de manifiesto en diversos estudios 44-47, 49-51, 55, 58 frente a otros en los que el seguimiento se realizó durante la estancia hospitalaria. 42,43 También existen estudios farmacoeconómicos comparando la HBPM y el pentasacárido de seguimiento a corto plazo dando a éste último mejor relación coste-efectividad. Se obtuvó un menor coste medio, 406 frente a $445 \$$ para enoxaparina, y menores eventos para fondaparinux: tanto en TVP $(2,4$ vs 5,4\%) como en TEP $(0,2 \text { vs } 0,8 \%)^{48}$

El presente estudio adolece sin duda de algunas limitaciones. En efecto, ante la escasez de datos empíricos sobre los efectos de la ETV y su tratamiento a largo plazo se han realizado múltiples supuestos que pueden haber sesgado los resultados. No se encontraron datos publicados de los sucesos ocurridos en tromboprofilaxis extendida con fondaparinux tras intervención de cada una de las COM: prótesis de cadera, prótesis de rodilla y fractura de cadera. Tan sólo se encontraron datos en un horizonte temporal alto de cinco años para los pacientes intervenidos de fractura de cadera con fondaparinux, ${ }^{61}$ por lo que han sido utilizados también para enoxaparina.

El análisis no consideró reducciones en calidad de vida que los pacientes sufren cuando tienen un episodio de ETV, que sí ha sido tenido en cuenta en otros estudios similares. ${ }^{13,23,55}$ Sin embargo, la no inclusión de las posibles pérdidas de calidad de vida en el modelo es una actitud conservadora, ya que favorecería en el análisis coste-efectividad a la alternativa dominada (enoxaparina), porque con fondaparinux la probabilidad de sufrir estos sucesos es menor.

En el modelo se ha excluido la probabilidad de supervivencia de cada cohorte de pacientes. La supervivencia estimada como años de vida ganados ha sido incluida previamente en otras evaluaciones económicas, ${ }_{13}^{13,21,53,54,56,58}$ pero aunque se hubiera tenido en cuenta en nuestro estudio no cambia la elección final, como sucede en los estudios mencionados. Lo mismo ocurre cuando se estiman directamente las muertes producidas o evitadas en cada grupo de pacientes. ${ }^{7,15,19,44,45,49,50,52}$ Por otra parte, la inclusión de la mortalidad en el árbol de decisión se acerca más a la realidad y se debería tener presente porque es un resultado en sí mismo. Su inclusión afectaría negativamente a la opción que menos por- centaje de muertes producidas debidas a episodios de ETV en el análisis del ratio coste-efectividad incremental o medio. En efecto, como ocurre en otros estudios que comparaban estrategias tromboprofilácticas tradicionales el ratio coste efectividad fluctuó desde $90 £$ (año 2002) por TEP fatal ahorrado, ${ }^{44}$ a $239 €$ (año 2006) por evento trombótico, ${ }^{45}$ o de $162,3 €$ (año 2006) por año de vida ganado al año de seguimiento. ${ }^{58}$

Aunque la mayoría de autores recomiendan tomar la perspectiva social en los análisis coste-efectividad de tal manera que se obtengan todas las consecuencias de las tecnologías utilizadas así como todos los recursos consumidos, la mayoría de las evaluaciones económicas sobre las consecuencias de la tromboprofilaxis en COM no lo hacen y se centran en la perspectiva del proveedor o del financiador de servicios sanitarios debido a la complejidad del registro de los acontecimientos o eventos producidos y del largo tiempo de seguimiento requerido. Los costes tomados suelen ser únicamente los directos médicos, no teniéndose en cuenta los indirectos ni los intangibles $44,45,49,58,59$

El análisis de sensibilidad realizado (univariante y multivatiante) confirma los resultados obtenidos del escenario base, incluso variando los parámetros seleccionados a extremos claramente en contra de la opción de fondaparinux. Al no hacer cambiar la decisión al variar los parámetros en el análisis de sensibilidad se reafirma la robustez de la decisión tomada. Los resultados de los análisis de sensibilidad también muestran congruencia interna entre los mismos, sobre todo con las probabilidades estimadas.

Estudios adicionales son necesarios que ayuden a confirmar estos hallazgos, es decir la dominancia de fondaparinux frente a enoxaparina en la tromboprofilaxis prolongada de los pacientes operados de fractura de cadera, así como determinar la magnitud de las diferencias de costes encontradas, y el ahorro exacto que pueda suponer al proveedor de servicios, en nuestro caso el Sistema Nacional de Salud.

No se han realizado ensayos clínicos controlados que comparen ambas estrategias profilácticas en pacientes sometidos a artroplastia total de cadera o de rodilla. Estudios realizados con metodología contrastada en este campo también ayudarán a aclarar las incertidumbres planteadas, y permitirán comparar los distintos subgrupos de pacientes sometidos a COM, como ya se ha realizado anteriormente en otros planteamientos tromboprofilácticos, ${ }^{44-47,49,50}$ asentando la evidencia obtenida y pudiéndose extrapolar los resultados a otros escenarios.

Otro aspecto que podría ser interesante estudiar en el futuro es la dosificación $60 \mathrm{mg}$ s.c. diarios de enoxaparina, repartidos en dos dosis o en dosis única, ya que parece ser más coste efectiva que la pauta de $40 \mathrm{mg}$ diarios auque no se han encontrado diferencias estadísticas significativas en su eficacia. ${ }^{42}$

Sería también interesante comparar la eficiencia del pentasacárido frente a los nuevos fármacos antitrombóticos orales, dabigatran y rivaroxaban, en fractura de cadera ya se disponen de evidencias prometedoras. Se debe 
Resultados y discusión

tener presente que todavía no han recibido su aprobación para usarse en esta indicación en su ficha técnica, aunque sí en la profilaxis en pacientes adultos sometidos a cirugía electiva de reemplazo de cadera o rodilla. ${ }^{37,65}$

La inclusión de los costes indirectos, es decir aquellos debidos al absentismo laboral de los pacientes, pueden resultar muy útiles en las evaluaciones económicas realizadas en enfermedades muy invalidantes o con secuelas muy graves como sucede en la ETV. Es un campo poco explorado en los análisis farmacoeconómicos de la tromboprofilaxis tras COM, por lo que sería de interés su investigación.

La magnitud de las diferencias encontradas entre ambas estrategias, más de $900 €$ por paciente, deben de ser confirmadas con posterioridad pero abre el debate de realizar un correcto seguimiento de enfermedades crónicas o que se pueden cronificar, como es la ETV.

Podemos concluir que la tromboprofilaxis extendida de la ETV tras fractura de cadera con fondaparinux $2,5 \mathrm{mg} /$ dia durante cuatro semanas evidencia una relación coste-efectividad dominante a $40 \mathrm{mg}$ diarios enoxaparina durante seis semanas. El fondaparinux se presenta como una alternativa más coste-efectiva que la enoxaparina, independientemente de las probabilidades de los eventos tanto los ETV, sintomáticos y no sintomáticos, la tasa de descuento y las hemorragias mayores, así como en el seguimiento a largo plazo. $\mathrm{AF}$

\section{BIBLIOGRAFIA}

1. Hansson PO, Sorbo J, Ericsson H. Recurrent venous thromboembolism alter deep vein trombosis: incidente and risk factors. Arch Intern Med 2000; 160: 769-74.

2. Oger E, Bressollette L, Nonent M, Lacut K, Guias B, Couturaud $\mathrm{F}$ y col. High prevalence of asymptomatic deep vein thrombosis on admission in a medical unit among elderly patients. Thromb Haemost 2002; 88: 592-7.

3. Hirsh J, Dalen J, Guyatt G; American College of Chest Physicians. The sixth (2000) ACCP guidelines for antithrombotic therapy for prevention and treatment of thrombosis. American $\mathrm{Co}-$ llege of Chest Physicians. Chest 2001; 119: 1S-2S.

4. Prandoni P, Lesing AW, Cogo A. The long-term clinical course of acute deep venous thrombosis. Arch Intern Med 1996; 125 : 1-7.

5. Granero Xiberta J. Grupo de Estudio del Tromboembolismo de la SECO'T. Guía Clínica: Profilaxis de la enfermedad tromboembólica en la cirugía mayor ortopédica traumatológica. Madrid: Sociedad Española de Cirugía Ortopédica y Traumatología; 2003.

6. O'Brien BJ, Anderson DR, Goeree R. Cost-effectiveness of enoxaparin versus warfarin prophylaxis against deep-vein thrombosis after total hip replacement. CMAJ 1994; 150: 1083-90.

7. Menzin J, Colditz GA, Regan MM, Richner RE, Oster G. Costeffectiveness of enoxaparin vs low-dose warfarin in the prevention of deep-vein thrombosis after total hip replacement surgery. Arch Intern Med 1995; 155: 757-64.

8. Hull RD, Raskob GE, Pineo GF, Feldstein W, Rosenbloom D, Gafni A y Col. Subcutaneous low-molecular-weight heparin vs warfarin for prophylaxis of deep vein thrombosis after hip or knee implantation: an economic perspective. Arch Intern Med 1997; 157: 298-303.

9. Hawkins DW, Langley PC, Krueger KP. A pharmacoeconomic assessment of enoxaparin and warfarin as prophylaxis for deep vein thrombosis in patients undergoing knee replacement surgery. Clin Ther 1998; 20: 182-95.
10. Francis CW, Pleil AM, Reinhart SP, Cohen B. A pharmacoeconomic evaluation of low-molecular-weight heparin in patients after total hipreplacement surgery. P and T 1999; 24: 136-45.

11. Wade WE, Chisholm MA. Cost effectiveness of deep venous thrombosis prophylaxis after hip fracture. Am J Orthop 2000; 29: 397-9.

12. Wade WE, Hawkins DW. Cost effectiveness of outpatient anticoagulant prophylaxis after total hip arthroplasty. Orthop 2000;23 335-8

13. Botteman MF, Caprini J, Stephens JM, Nađipelli V, Bell CF, Pashos CL y Col. Results of an economic model to assess the costeffectiveness of enoxaparin, a low-molecular-weight heparin, versus warfarin for the prophylaxis of deep vein thrombosis and associated long-term complications in total hip replacement surgery in the United States. Clin Ther 2002; 24: 1960-86.

14. Caprini JA, Arcelus J, Kudrna JC, Sehgal LR, Oyslender M, Maksimovic D y Col. Cost-effectiveness of venous thromboembolism prophylaxis after total hip replacement. Phlebology 2002; 17: 126-33.

15. Nerurkar J, Wade WE, Martin BC. Cost/death averted with venous thromboembolism prophylaxis in patients undergoing total knee replacement or knee arthroplasty. Pharmacotherapy 2002; 22: $990-1000$.

16. Sarasin FP, Bounameaux H. Out of hospital antithrombotic prophylaxis after total hip replacement: low-molecular-weight heparin, warfarin, aspirin or nothing? Thromb Haemost 2002; 87 : 586-92.

17. Dahl OE, Pleil AM. Investment in prolonged thromboprophylaxis with dalteparin improves clinical outcomes after hip replacement. J Thromb Haemost 2003; 1: 896-906.

18. Levin LA, Bergqvist D. Cost effectiveness of desirudin compared with a low molecular weight heparin in the prevention of deep vein thrombosis after total hip replacement surgery. Pharmacoecónomics 2001; 19: 589-97.

19. Mol EM, Egberts TCG. Prophylaxis for venous thromboembolism in hip fracture surgery. Pharmacoeconomics 1994; 5: 48-55.

20. Wade WE. Cost effectiveness of danaparoid compared with enoxaparin as deep vein thrombosis prophylaxis after hip replacement surgery. Am J Orthop 1999; 28: 229-31.

21. Lynd LD, Goeree R, Crowther MA, O'Brien BJ, A probabilistic cost-effectiveness analysis of enoxaparin versus unfractionated heparin for the prophylaxis of deep-vein thrombosis following cop

2. Lassen MR, Borris LC, Anderson BS, Jensen HP, Skejø Bro HP, Andersen $\mathrm{G}$ y cols. Efficacy and safety of prolonged thromboprophylaxis with a low molecular weight heparin (dalteparin) after total hip arthroplasty - the Danish Prolonged Prophylaxis (DaPP) Study. Thromb Res 1998; 89: 281-7.

23. Marchetti M, Liberato NL, Ruperto N, Barosi G. Long-term costeffectiveness of low molecular weight heparin versus unfractionated heparin for the prophylaxis of venous thromboembolism in elective hip replacement. Haematologica 1999; 84: 730-7.

24. Hull RD, Pineo GF. Extended prophylaxis against venous thromboembolism following total hip and knee replacement. Haemostasis 1999; 29 (S1): 23-31.

25. Comp PC, Spiro TE, Friedman RJ, Whitsett TL, Johnson GJ, Gardiner GA Jr y cols. Enoxaparin Clinical Trial Group. Prolonged enoxaparin therapy to prevent venous thromboembolism after primary hip or knee replacement. Enoxaparin Clinical Trial Group. J Bone Joint Surg Am 2001; 83: 336-45.

26. Hull RD, Pineo GF, Stein PD, Mah AF, MacIsaac SM, Dahl OE y cols. Extended out-of-hospital low-molecular-weight heparin prophylaxis against deep venous thrombosis in patients after elective hip arthroplasty: a systematic review. Ann Intern Med 2001; 135: 858-69. 
Resultados y discusión

27. Eikelboom JW, Quinlan DJ, Douketis JD. Extended-duration prophylaxis against venous thromboembolism after total hip or knee replacement: a meta-analysis of the randomised trials. Lancet 2001; 358: 9-15.

28. Khushal A, Quinlan D, Alikhan R, Gardner J, Bailey C, Cohen A. Thromboembolic disease in surgery for malignancy-rationale for prolonged thromboprophylaxis. Semin Thromb Hemost 2002; 28: 569-76

29. Prandoni P, Bruchi O, Sabbion P, Tanduo C, Scudeller A, Sardella $\mathrm{C}$ y Col. Prolonged thromboprophylaxis with oral anticoagulants after total hip arthroplasty: a prospective controlled randolants after total hip arthroplasty: a prospective control
mized study. Arch Intern Med 2002; 162: 1966-71.

30. Kolb G, Bodamer I, Galster H, Seidlmayer C, Grambach K, Koudela K y cols. Long-term Thromboprophylaxis Study Group. Reduction of venous thromboembolism following prolonged prophylaxis with the low molecular weight heparin Certoparin prophylaxis with the low molecular weight heparin Certoparin after endoprothetic joint replacement or osteosynthesis of the
lower limb in elderly patients. Thromb Haemost 2003; 90: 1100-5.

31. O'Donnell M, Linkins LA, Kearon C, Julian J, Hirsh J. Reduction of out-of-hospital symptomatic venous thromboembolism by exten the tended thromboprophylaxis with low-molecular-weight heparin following elective hip arthroplasty: a systematic review. Arch Intern Med 2003: 163: 1362-6.

32. Geerts WH, Pineo GF, Heit JA, Bergqvist D Lassen MR, Colwell $\mathrm{CW}$ y cols. Prevention of venous thromboembolism: the Seventh ACCP Conference on Antithrombotic and Thrombolytic Therapy. ACCP Conference on Antithro

33. Huisman MV, Bounameaux H. Treating patients with venous thromboembolism: initial strategies and long-term secondary prevention. Semin Vasc Med 2005; 5: 276-84.

34. Kher A, Samama MM. Primary and secondary prophylaxis of venous thromboembolism with low-molecular-weight heparins: prolonged thromboprophylaxis, an alternative to vitamin $\mathrm{K}$ antagonists. J Thromb Haemost 2005; 3: 473-81.

35. Rasmussen MS, Jorgensen LN, Wille-Jørgensen P, Nielsen JD, Horn A, Mohn AC y Col; FAME Investigators. Prolonged prophylaxis with dalteparin to prevent late thromboembolic complications in patients undergoing major abdominal surgery: a multicenter randomized open-label study. J Thromb Haemost 2006; 4 2384-90.

36. Dorr LD, Gendelman V, Maheshwari AV, Boutary M, Wan Z, Long WT. Multimodal thromboprophylaxis for total hip and knee arthroplasty based on risk assessment. J Bone Joint Surg Am 2007; 89: 2648-57.

37. Eriksson BI, Dahl OE, Rosencher N, Kurth AA, van Dijk CN, Frostick SP, y Col. RE-NOVATE Study Group. Dabigatran etexilate versus enoxaparin for prevention of venous thromboembolate versus enoxaparin for prevention of venous thromboembolism after total hip replacement: a randomised,

38. Lapidus LJ, Ponzer S, Elvin A, Levander C, Lärfars G, Rosfors S, de Bri E. Prolonged thromboprophylaxis with Dalteparin during immobilization after ankle fracture surgery: a randomized placebocontrolled, double-blind study. Acta Orthop 2007; 78: 528-35.

39. Lubenow N, Hinz P, Ekkernkamp A, Greinacher A. Should patients be informed about the risk of heparin-induced thrombocytopenia before prolonged low-molecular-weight heparin thromboprophylaxis post-trauma/orthopedic surgery?. Eur J Haematol 2007; 79: $187-90$.

40. Otero-Fernández R, Gómez-Outes A, Martínez-González J, Rocha E, Fontcuberta J; Bemiparin Cooperative Study Group in Orthopaedic Patients. Evaluation of the effectiveness and safety of bemiparin in a large population of orthopedic patients in a norma bemiparin in a large population of orthopedic patients in a normal

41. Eriksson BI, Bauer KA, Lassen MR, Turpie AG; Steering Committee of the Pentasaccharide in Hip-Fracture Surgery Study. Fondaparinux compared with enoxaparin for the prevention of venous thromboembolism after hip-fracture surgery. $\mathrm{N}$ Engl $\mathbf{J}$ Med 2001; 345: 1298-304.

42. Wade WE, Spruill WJ, Leslie RB. Cost analysis: fondaparinux versus preoperative and postoperative enoxaparin as venous thromboembolic event prophylaxis in elective hip arthroplasty. Am J Orthop 2003; 32: 201-5.

43. Wade WE, Spruill WJ, Leslie RB. Cost analysis of fondaparinux versus enoxaparin as venous thromboembolism prophylaxis in hip Venous Thromboembolism prophylaxis fracture surgery. Am J Ther 2004; 11: 194-8.

44. Gordois A, Posnett J, Borris P, Bussuyt P, Jönsson B, Levy E, De Pouvourville $\mathrm{G}$. The cost-effectiveness of fondaparinux compared with enoxaparin as prophylaxis against thromboembolism following major orthopedic surgery. J Thromb Haemost 2003; 1 $2167-74$.

45. Lundkvist J, Bergqvist D, Jonsson B. Cost-effectiveness of fondaparinux vs. enoxaparin as venous thromboembolism prophylaxis in Sweden. Eur J Health Econ 2003; 4: 254-62.

46. Annemans L, Minjoulat-Rey MC, De Knock M, Vranckx K, Czarka M, Gabriel S y Col. Cost consequence analysis of fondaparinux versus enoxaparin in the prevention of venous thromboembolism after major orthopaedic surgery in Belgium. Acta Clin Belg 2004; 59: 346-57.

47. Dranitsaris G, Kahn SR, Stumpo C, Paton TW, Martineau J, Smith $R$ y cols. Pharmacoeconomic analysis of fondaparinux versus enoxaparin for the prevention of thromboembolic events in orthopedic surgery patients. Am J Cardiovasc Drugs 2004; 4 325-33.

48. Spruill WJ, Wade WE, Leslie RB. A cost analysis of fondaparinux versus enoxaparin in total knee arthroplasty. Am J The 2004; $11: 3-8$.

49. Sullivan SD, Davidson BL, Kahn SR, Muntz JE, Oster G, Raskob G. A cost-effectiveness analysis of fondaparinux sodium compared with enoxaparin sodium as prophylaxys against venous thromboembolism: use in patients undergoing major orthopaedic surgery. Pharmacoeconomics 2004; 22: 605-20.

50. Bjorvatn A, Kristiansen F. Fondaparinux sodium compared with enoxaparin sodium: a cost-effectiveness analysis. Am J Cardiovasc Drugs 2005; 5: 121-30.

51. Sullivan SD, Kwong L, Nutescu E. Cost-effectiveness of fondaparinux compared with enoxaparin as prophylaxis against venous thromboembolism in patients undergoing hip fracture surgery. Value Health 2006; 9: 68-76.

52. Detournay B, Planes A, Vochelle N, Fagnani F. Cost effectiveness of a low-molecular-weight heparin in prolonged prophylaxi against deep vein thrombosis after total hip replacement. Pharmacoeconomics $1998 ; 13 ; 81-9$.

53. Bergqvist D, Jönsson B. Cost-effectiveness of prolonged administration of a low molecular weight heparin for the preven ion of deep venous thrombosis following total hip replacement. 'alue Health 1999; 2: 288-94.

54. Davies LM, Richardson GA, Cohen AT. Economic evaluation of enoxaparin as postdischarge prophylaxis for deep vein thrombosis (DVT) in elective hip surgery. Value Health 2000; 3: 397-406.

55. Haentjens P, De Gropte K, Annemans L. Prolonged enoxaparin therapy to prevent venous thromboembolism after primary hip or knee replacement A cost-utility analysis. Arch Orthop Trauma Surg 2004; 124: 507-17.

56. Bischof M, Leuppi JD, Sendi P. Cost-effectiveness of extended venous thromboembolism prophylaxis with fondaparinux in hip surgery patients. Exp Rev Pharmacoeconomics Outomes Res 2006; 6: 171-80.

57. Friedman RJ, Dunsworth GA. Cost analyses of extended prophylaxis with enoxaparin after hip arthroplasty. Clin Orthop Relat Res 2000; 370: 171-82. 
Resultados y discusión

58. Lundkvist J, Bergqvist D, Jönsson B. Cost-effectiveness of extended prophylaxis with fondaparinux compared with low molecular weight heparin against venous thromboembolism in patients undergoing hip fracture surgery. Eur J Health Econ 2007 tients undergo $313-23$.

59. Slef J, Badia X. Evaluación clínica y económica de la profilaxi con fondaparinux en comparación con enoxaparina en cirugía ortopédica mayor. Pharmacoeconomics-Spanish Research Articles topédica mayor

60. Sullivan SD, Kahn SR, Davidson BL, Borris L, Bossuyt P, Raskob G. Measuring the outcomes and pharmacoeconomic consequences of venous thromboembolism prophylaxis in major orthopaedic surgery. Pharmacoeconomics 2003; 21: 477-96.

61. Eriksson BI, Lassen MR; PENTasaccharide in HIp-FRActure Surgery Plus Investigators. Duration of prophylaxis against venous thromboembolism with fondaparinux after hip fracture surgery: a multicenter, randomized, placebo-controlled, double-blind study. Arch Intern Med 2003; 163: 1337-42.

62. Bauer KA, Eriksson BI, Lassen MR, Turpie AG; Steering Committee of the Pentasaccharide in Major Knee Surgery Study. Fondaparinux compared with enoxaparin for the prevention of venous thromboembolism after elective major knee surgery. $\mathrm{N}$ Engl J Med 2001; 345: 1305-10.

63. Lassen MR, Bauer KA, Eriksson BI, Turpie AG; European Pentasaccharide Elective Surgery Study (EPHESUS) Steering Committee. Postoperative fondaparinux versus preoperative enoxaparin for prevention of venous thromboembolism in elective the hip-replacement surgery: a randomised double-blind comparison.
64. Turpie AG, Bauer KA, Eriksson BI, Lassen MR; PENTATHALON 2000 Study Steering Committee. Postoperative fondaparinux versus postoperative enoxaparin for prevention of venous thromboembolism after elective hip-replacement surgery: a randomised double-blind trial. Lancet 2002; 359: 1721-6.

65. Agencia española de medicamentos y productos sanitrarios. Disponible en Internet: https://sinaem4.agemed.es/consaem/fichaspocnicas.do?metodo=buscar [Consulta: 22 octubre 2009] 


\section{Capítulo 6}

Carrera-Hueso FJ, Poquet Jornet JE, Ramón Barrios A, Conde Fernández F. Coste-efectividad en la inducción del parto con dinoprostona. Aten Farm 2010; 12 (6): 359-68. 
Resultados y discusión

\section{COSTE-EFECTIVIDAD \\ EN LA INDUCCIÓN DEL PARTO CON DINOPROSTONA}

Carrera Hueso Francisco Javier, Poguet Jornet Jaime Eduardo, Ramón Barrios Auxiliadora, Conde Fernández Fernando

\section{RESUMEN}

Objetivo: Determinar cuál de las dos presentaciones de dinoprostona, gel endocervical y dispositivo intrauterino, a dosis usuales, presenta mejor relación costeefectividad para la maduración del cérvix antes de la inducción del parto.

Método: Se diseñó un árbol de decisión que recoge los efectos de los fár macos y sus eventos adversos. La perspectiva utilizada es la del hospital. El horizonte temporal fue menor a un año. No se han contemplado costes indirectos ni intangibles, solamente los costes directos sanitarios en cada alternativa (en euros del 2010). Se procedió a un análisis de sensibilidad, uni y bivariante, haciendo variar ampliamente los parámetros fundamentales del modelo alrededor de la situación basal, y se procedió a testar la solidez del modelo cambiando su estructura.

Resultados: Prepidil $0,5 \mathrm{mg}$ gel endocervical administrado dos veces al día es una opción dominada $(\mathrm{C} / \mathrm{E}=8,97)$ por una dosis $10 \mathrm{mg}$ de Propess $^{\circledR}$ vía vaginal para la maduración del cérvix antes de la inducción del parto en mujeres gestantes nulíparas $(\mathrm{C} / \mathrm{E}=1,46)$. Los resultados del análisis de sensibilidad univariado y bivariante de todos los parámetros testados muestran la robustez del modelo. Los mismos resultados se obtuvieron con el análisis de sensibilidad estructural.

Conclusión: El tratamiento de las gestantes a termino nulíparas con el cuello uterino inmaduro con una dosis Propess ${ }^{\circledR} 10 \mathrm{mg}$ de liberación vaginal (dinoprostona) presenta una relación más favorable coste efectiva que dos dosis separadas seis horas entre sí de Prepidil ${ }^{\circledR} 0,5 \mathrm{mg}$ gel endocervical $(2,5 \mathrm{ml})$.

DinOPROSTONA - INDUCCIÓN DEL PARTO - MADURACIÓN DEL CÉRVIX -

COSTE-EFECTIVIDAD - Gel VAGiNAL - Dispositivo INTRAUTERINO

\section{ABSTRACT}

Objective: To determine which one of the two forms of dinoprostone, endocervical gel and intrauterine device, at typical doses, has a better cost-effectiveness relation for cérvix maturation before labor induction.

Method: We designed a decision tree that captured the effects of drugs and their adverse events. The hospital's perspective was the one being used. The time horizon was less than a year. No indirect or intangible costs were considered, only those direct health costs for each alternative (in 2010 Euros). We performed a univariate and bivariate sensitivity analysis by extensively changing the model's fundamental parameters around the basal situation, and proceeded to test the model's solidity by changing its structure.

Results: $0.5 \mathrm{mg}$ Prepidil endocervical gel administered twice a day is an option $(C / E=8.97)$ dominated by a $10 \mathrm{mg}$ Propess ${ }^{\circledR}$ dose by vaginal way for cérvix maturation before labor induction in nulliparous pregnant women (C/E $=1.46$ ). The univariate and bivariate sensitivity analysis results of all tested parameters proved the model's robustness. The same results were obtained with the structural sensitivity analysis. 
Resultados y discusión

Conclusion: The treatment of nulliparous pregnant women at term with immature uterine neck with a dose of $10 \mathrm{mg}$ Propess ${ }^{(1)}$ of vaginal liberation (dinoprostone) showed a more favorable cost-effectiveness relation than two doses six hours apart of $0.5 \mathrm{mg}$ Prepidil ${ }^{(8)}$ endocervical gel $(2.5 \mathrm{ml})$.

DINOPROSTONE - LABOR INDUCTION - CÉRVIX MATURATION - COST-EFFECTIVENESS VAGINAL GEL - INTRAUTERINE DEVICE

\section{INTRODUCcIóN}

La inducción del parto se realiza en aquellas circunstancias en que se piensa que la finalización voluntaria del embarazo es más beneficiosa que dejar que éste siga su curso natural. Es quizás esta inducción la única tecnología médica disponible que busca adelantar un proceso el cual sería inevitable a menos que el embarazo se termine por cesárea o la madre muera antes de dar a luz. ${ }^{1}$

El impacto emocional sobre la madre que tiene la inducción del parto es indudable pero sin embargo ha sido evaluada en contadas ocasiones; además puede ser menos eficiente y generalmente más dolorosa que el parto espontáneo. También suele requerir el parto con inducción, más analgesia epidural y requiere un mayor grado de intervenciones durante el parto (partos instrumentales)..$^{1-5}$ Cuando se induce el parto, aproximadamente menos de dos tercios de las mujeres paren sin intervención médica, unas $15 \%$ tienen partos instrumentales y un $22 \%$ acaban en cesáreas urgentes ${ }^{6-8}$

Existe un grupo de pacientes, las nulíparas con cérvix desfavorable, que representan un reto para los clínicos pues ambas condiciones, la inducción y maduración del cérvix, están asociadas con una tasa de fracaso de inducción mayor y por lo tanto de un aumento en la tasa de cesáreas. ${ }^{9}$

La maduración cervical es un proceso activo en el cual el cérvix cambia de forma y consistencia como resultado de la degradación del colágeno por las proteasas y peptidasas. ${ }^{10}$

Para considerar y ofrecer la inducción a una gestante a término es preciso que exista evidencia de que la intervención acarrea beneficios tanto para la madre como para el feto, esto requiere un análisis cuidadoso de la evidencia clínica así como plantear previamente el tema con la madre. La gestante debe ser informada, por parte de su ginecólogo, que la mayoría las mujeres iniciaran el parto espontáneo antes de las 42 semanas de gestación. Está bien establecido que la mujer y su pareja necesiten información para decidir sobre los cuidados a recibir, y la inducción al parto no es ninguna excepción. ${ }^{11}$

Se debe evaluar en primer lugar el estado del cérvix de la gestante mediante un examen vaginal usando el Test de Bishop (Tabla 1). ${ }^{12}$ El resultado del test ayuda a relacionar cuantitativamente el estado del cérvix con el grado de éxito de la inducción y la duración del parto. Prácticamente en casi todos los estudios se intenta la maduración del cérvix cuando el resultado del test de Bishop es menor a 4, y también se ha utilizado para valorar la efícacia de los medicamentos al cabo de 12 ó 24 horas si se producía un aumento del valor inicial registrado en las gestantes.

Existen evidencias epidemiológicas robustas que indican un mayor riesgo, tanto para la madre como para el feto, cuando el embarazo se prolonga más allá de la 40 semana. ${ }^{13-21} \mathrm{El}$ parto debe presentarse antes de finalizada la 42 semana de gestación debido al incremento en el riesgo de mortalidad perinatal y de morbilidad asociadas con retrasos del momento del parto. ${ }^{22}$

Definimos embarazo prolongado como aquel que se prolonga más allá de la semana 42 de gestación cuando ésta ha sido datada mediante ecografía en el primer trimestre ${ }^{23}$ (5-10\% de embarazos $\left.{ }^{24}\right)$. Las recomendaciones a seguir para la inducción de parto prolongado serían las siguientes: ${ }^{25,26}$

- Toda mujer con un embarazo prolongado debe tener la oportunidad de empezar el parto de manera espontánea. A estas mujeres debería ofrecérsele la inducción del parto entre las semanas 41 y 42 para evitar los riesgos que conlleva el embarazo prolongado. El momento exacto para la inducción debe tomar en consideración las preferencias de la madre y las circunstancias locales del hospital. 8,17

- Si una mujer decide no ser inducida, respetando el principio bioético de autonomía, su decisión debe ser respetada.

- A partir de la semana 42 una mujer que declina la inducción de parto, debe ser monitorizada como mínimo dos veces a la semana así como realizarle una estimación del índice de líquido amniótico.14, 17. 19-26

Múltiples métodos han sido desarrollados para la dilatación del cérvix, preparándolo para la inducción con oxitocina y promover el parto vaginal.

a) Métodos mecánicos: ${ }^{27-29}$ amniotomía (ruptura artificial de membranas), balón de Foley, laminaria o dilatadores progresivos han sido utilizados exitosamente, pero pueden traumatizar el cérvix y predisponer a infecciones posteriores. La amniotomía ha obtenido dudosos resultados en cérvix inmaduro (rígido o firme).

b) Agentes farmacológicos: ${ }^{27-40}$ estrógenos, relaxina y prostaglandinas. Recientemente se ha utilizado, para la maduración del cérvix, comprimidos vaginales de misoprostol (Misofar ${ }^{\sqrt{ }}$ ), análogo sintético de la prostaglandina $E_{1}$, de manera muy beneficiosa, y mifepristona, un antagonista de la progesterona. Desde la aparición de las prostaglandinas para la maduración 
Resultados y discusión

TABLA 1. Test de Bishop.

\begin{tabular}{|c|c|c|c|c|}
\hline Parámetro a evaluar & $\begin{array}{l}\text { Puntuación } \\
0\end{array}$ & 1 & 2 & 3 \\
\hline Dilatación $(\mathrm{cm})$ & Cerrado & $1-2$ & $3-4$ & $>5$ \\
\hline Borramiento (\%) & $0-30$ & $40-50$ & $60-70$ & $>80$ \\
\hline Presentación & & & & \\
\hline (relación con espinas ischiales) & -3 & -2 & $-1 \circ 0$ & $+1,+2$ \\
\hline Consistencia & Firme & Media & Blando & - \\
\hline Posición del cerviz & Posterior & Media & Anterior & - \\
\hline
\end{tabular}

del cérvix, la inducción del parto se ha popularizado, debido a que incrementan la probabilidad de parto vaginal, disminuye el intervalo de tiempo para la iniciación del parto y disminuye la cantidad máxima utilizada de oxitocina para la inducción.

Estos efectos beneficiosos ya fueron constatados en 1978 en los estudios que utilizaban preparaciones magistrales de dinoprostona gel realizadas por los servicios farmacéuticos hospitalarios. ${ }^{39,40}$ Actualmente están comercializadas en nuestro país dos presentaciones de la dinoprostona (Prostaglandina $\mathrm{E}_{2}$ ), de aplicación local vía endocervical o vaginal, y son:

- Prepidil ${ }^{\mathbb{Q}} 0,5 \mathrm{mg}$ gel endocervical. ${ }^{41}$

_. Propess $^{\circledR} 10 \mathrm{mg}$ sistema de liberación vaginal. ${ }^{42}$

Diferentes estudios han sido realizados comparando las vías de administración del análogo de la prostaglandina $E_{2}$ para la maduración del cérvix previa inducción o no con oxitocina posterior. ${ }^{40,43-57}$ Los resultados obtenidos no han sido concluyentes a favor de ninguna de las dos presentaciones tal como se refleja en diversos meta-análisis publicados por la elevada heterogeneidad de los mismos al emplearse diferentes dosis administrada, procedencia variable de los medicamentos, ge comercializado o preparación magistral, entre otros factores. ${ }^{43}, 51,53,57$ De los dos estudios farmacoeconómicos tradicionales realizados al respecto, tampoco han aportado evidencia a favor de una presentación frente a la otra porque o bien no contemplaron las náuseas y vómitos ${ }^{44}$ o bien no incluyeron las hemorragias post-parto. ${ }^{45}$ Además, este último estudio no diferenció entre parto vaginal natural e instrumental. ${ }^{45}$

No se han realizado estudios que comparen ambas presentaciones a pesar de su impacto presupuestario directo. ${ }^{46} \mathrm{El}$ Propess ${ }^{(B)}$ cuesta más de dos veces y media que el Prepidil ${ }^{\mathbb{Q}}$ gel, lo que a priori nos podría inclinar a seleccionar la presentación de Prepidil ${ }^{(\mathbb{1})}$ gel por ser la más económica sin evaluar si es la opción más favorable, para lo que se tendría que realizar un estudio farmacoeconómico en el que se incluyan todos los otros costes implicados en el proceso.

El principal objetivo del presente trabajo es determi- nar cuál de las dos presentaciones de dinoprostona, gel endocervical y dispositivo intrauterino, a dosis usuales, presenta mejor relación coste-efectividad para la maduración del cérvix antes de la inducción del parto.

\section{MÉTODO}

Alternativas comparadas y perspectivas. Se realiza un estudio coste-efectividad entre las dos presentaciones de dinoprostona, al objeto de valorar cuál presenta una relación coste-efectividad más favorable, para ello se diseñó un modelo, árbol de decisión, a propósito de tal manera que se recojan todos los efectos de los fármacos así como los eventos adversos asociados con su administración.

Los tratamientos comparados en mujeres previamente seleccionadas (gestantes nulíparas y con un valor del test de Bishop de cuatro o menor ${ }^{\cdot 12}$ ) fueron:

a) administración de Prepidil ${ }^{\circledR} 0,5 \mathrm{mg}$ gel endocervical $(2,5 \mathrm{ml})$, dos dosis separadas seis horas entre sí;

b) una dosis única de Propess ${ }^{(1)} 10 \mathrm{mg}$ sistema de liberación vaginal.

La perspectiva utilizada en el estudio es la del proveedor de servicios sanitarios, que en este caso es el hospital.

El horizonte temporal es menor a un año, debido a inmediatez tanto de los resultados como de los efectos que se derivan de la utilización de los tratamientos, lo que significa no es necesaria la utilización de una tasa de descuento en los costes ni en los efectos de las alternativas.

Modelo utilizado y asunciones. Los programas informáticos utilizados en el diseño, desarrollo y resolución del modelo propuesto para el estudio coste-efectividad han sido: Microsoft Office Excel 2003 y Data 3.5 (TreeAge Software 1998).

Se ha utilizado un modelo de análisis de decisión que contempla todos los resultados posibles en mujeres parturientas. Para la resolución del modelo se ha empleado el programa TreeAge, ayudado aritméticamente por Microsoft Office Excel.

Para favorecer la lectura del árbol de decisión, se presenta un árbol de decisión colapsando todas las ramas, 


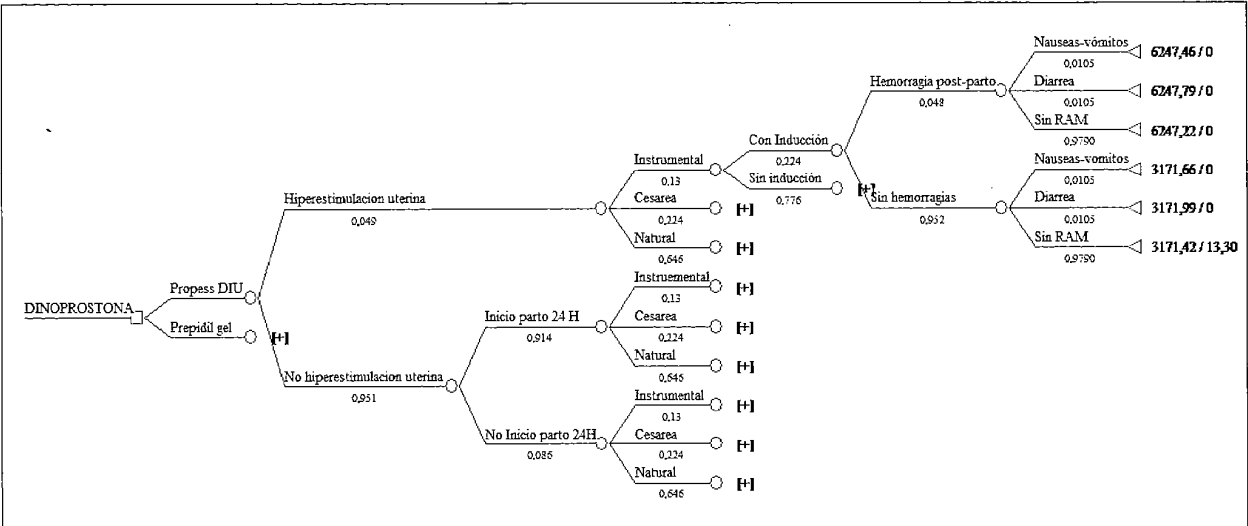

FIG. 1. Modelo de análisis de decisión, colapsando todas las ramas, excepto la primera y donde se resumen los resultados posibles en mujeres parturientas.

excepto la primera, obteniendo los resultados que se muestran en la Fig. 1.

Se contabilizaron como resultados tanto los partos vaginales (naturales o instrumentales) como las cesáreas. Se asumió que un parto instrumental ocasionaba un día más de hospitalización frente al parto vaginal natural

Los principales eventos adversos de la utilización de dinoprostona aplicada localmente (hemorragias postparto e hiperestimulación uterina) se recogen como variables independientes. Sobre los restantes efectos adversos del fármaco se optó por registrarlos conjuntamente en otra variable.

En el modelo se asume que para la maduración del cérvix es necesario el transcurso de 24 horas del inicio del tratamiento con prostaglandina $\mathrm{E}_{2}$ para valorar su eficacia. Es por ello por lo que se recogen las probabilidades de parto espontáneo o cesárea transcurrido ese tiempo. Se consideró que todas en las pacientes que presenten hiperestimulación o hipertono, se procede directamente a la instauración de las maniobras del parto, sin necesidad de esperar las 24 horas para valorar su efectividad. Las probabilidades a priori de la variable Inicio_24H se presentan en la Tabla 2.

Con el modelo presentado se obtienen 108 posibles resultados para cada una de las alternativas estudiadas.
Análisis coste-efectividad. No se han contemplado costes indirectos ni intangibles, solamente se toman en cuenta los costes directos sanitarios en cada alternativa. Todos los costes utilizados han sido expresados en euros del año 2010

Los costes de todos los medicamentos utilizados, tanto los de tratamiento como los de rescate, se ha utilizado el Precio de Venta al Público en el Catálogo de Especialidades Farmacéuticas 2010 del Consejo General de Colegios Oficiales de Farmacéuticos. El resto de las cantidades monetarias han sido obtenidas del servicio de farmacia del hospital.

Los costes por proceso, GRD ajustados, han sido facilitados por el servicio de contabilidad analítica de la Dirección de Gestión del Hospital Dr. José Molina Orosa. Otro GRD (hemorragias graves) ha sido extraído de una base de datos privada, SOIKOS. Los otros datos han sido calculados a partir de las asunciones del modelo realizadas.

Para conocer la efectividad de los tratamientos se tomó el número de gestantes que traían al bebe por parto vaginal, instrumental o natural, sin ningún tipo de complicación (ni hemorragia post-parto ni ninguna reacción adversa). Por tanto, se valora el número de partos vaginales sin efectos secundarios.

TABLA 2. Probabilidades a priori de la variable Inicio_24H. ${ }^{36,43,45,48,50}$

\begin{tabular}{lllll} 
P(Inicio_24H) & $\begin{array}{l}\text { PROPESS } \\
\text { Hiperestimulación } \\
\text { uterina }\end{array}$ & $\begin{array}{l}\text { Sinhiperestimutación } \\
\text { uterina }\end{array}$ & $\begin{array}{l}\text { PREPIDIL } \\
\text { Hiperestimulación } \\
\text { uterina }\end{array}$ & $\begin{array}{l}\text { Sin hiperestimulación } \\
\text { uterina }\end{array}$ \\
\hline Sí & 0 & 0,91 & 0 & 0,72 \\
No & 1 & 0,08 & 1 & 0,28 \\
\hline
\end{tabular}


Resultados y discusión

TABLA 3. Coste de los medicamentos (tanto para la maduración del cérvix e inducción del parto como para el tratamiento de las reacciones adversas) y de los procedimientos realizados.

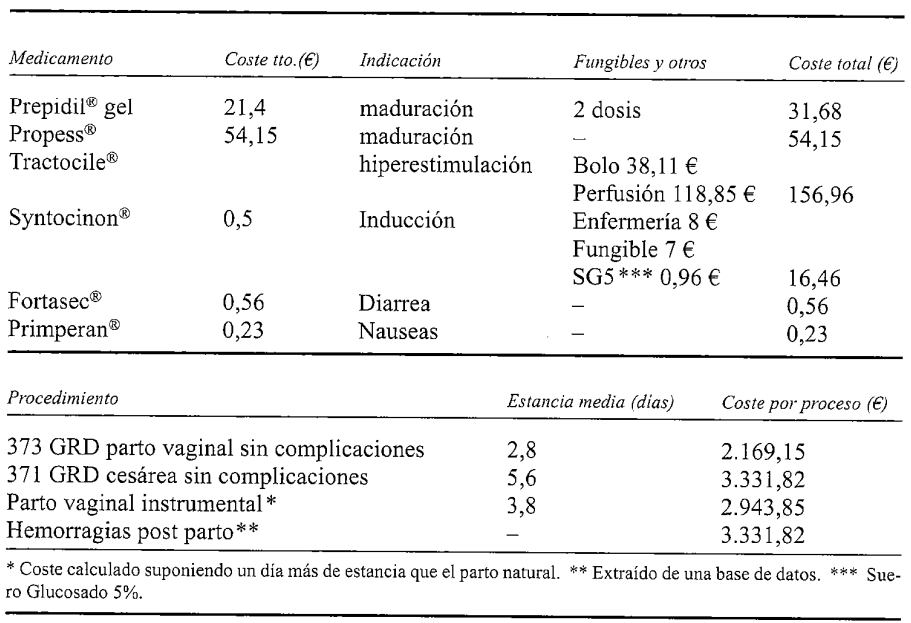

Para calcular el grado de variación de cada parámetro, coste y efectividad media, se realizó una simulación de Montecarlo con 10.000 reiteraciones al objeto de obtener el intervalo de confianza al $95 \%$ en cada una de las alternativas estudiadas.

Se calcula la razón coste efectividad de cada alternativa, es decir la relación $\mathrm{C} / \mathrm{E}$, y la mejor elección es la que tenga una relación menor. Es recomendable calcula también el ratio coste efectividad incremental para que proporcione mayor información al decidor.

Para valorar la relación coste efectividad de los tratamientos, se calculó el ratio coste efectividad incremental (RCEI) de una alternativa frente a la otra. El cálculo se realiza de la siguiente manera, (tomando A y B como alternativas)

$$
R C E I=\Delta C / \Delta E=C_{A}-C_{B} / E_{A}-E_{B}
$$

Siendo:

$\mathrm{C}_{\mathrm{A}}$ : costes de la alternativa $\mathrm{A}$

$\mathrm{C}_{\mathrm{B}}$ : costes de la alternativa $\mathrm{B}$

$E_{A}$ : eficacia o efectividad de la alternativa $A$

$E_{B}$ : eficacia o efectividad de la alternativa $B$

Nótese la diferencia de calcular los ratios promedios de cada alternativa y compararlos, es decir calcular $\mathrm{CA} / \mathrm{EA}$ y $\mathrm{CB} / \mathrm{EB}$ y compararlos entre ellos, a diferencia del RCEI que nos informa del coste monetario para conseguir una unidad adicional de eficacia.

Análisis de sensibilidad. En los resultados obtenidos con el modelo pueden influir varios factores que incorporan incertidumbre: a) la incidencia de los resultados o de los eventos adversos, se basa en probabilidades derivadas de estudios empíricos expuestos a variación aleatoria;

b) los datos de los ensayos clínicos controlados han sido obtenidos en condiciones ideales, que pueden no representan necesariamente la práctica clínica habitual;

c) en cuanto al consumo de recursos sanitarios, es susceptible de error de estimación, tanto por la cantidad empleada como por su coste unitario.

Por estas razones, se procedió a un análisis de sensibilidad haciendo variar ampliamente los parámetros fundamentales del modelo alrededor de la situación basal Se efectuó un análisis unifactorial con el fin de contrastar la robustez de los resultados variando las asunciones realizadas. Las suposiciones testadas fueron:

- Considerar como resultado positivo de los tratamientos los partos no complicados incluyendo a los definidos anteriormente, los que presentaron reacciones adversas leves (náuseas y vómitos o diarrea), que pueden considerarse clínicamente no relevantes.

- Considerar como éxito solamente los partos producidos antes de las 24 horas sin rcacciones adversas y sin complicaciones, es decir sin que se produjese hiperestimulación uterina ni posteriores hemorragias mayores.

- Duplicar el coste de la inducción cuando no se produce el parto antes de las 24 horas, ya que se necesitaría mayor cantidad de oxitocina para evitar el fracaso. En las ramas que proceda en lugar de poner $16,46 €$ sustituir por $32,92 €$ 
taBlA 4. Probabilidades del modelo obtenidas a priori y que sirvieron para diseñar el modelo.

\begin{tabular}{llll}
\hline Concepto & PREPIDIL & & \\
\hline gel & PROPESS & \\
Parto tras 24 horas inducción & 0,72 & 0,91 & 36 Funte \\
Parto vaginal natural & 0,32 & 0,56 & 36 \\
Cesárea & 0,36 & 0,22 & 36 \\
Parto instrumental & 0,34 & 0,13 & 36,47 \\
Inducción con oxitocina & 0,53 & 0,22 & 36 \\
Hiperestimulación uterina & 0,03 & 0,04 & $39,41,42,57$ \\
Náuseas y vómitos & 0,01 & 0,01 & 41,42 \\
Diarrea & 0,01 & 0,01 & 41,42 \\
Hemorragias post parto & 0,16 & 0,04 & 51 \\
\hline
\end{tabular}

TABLA 5. Probabilidades a priori del nodo de efectos adversos. ${ }^{36,50,51,53,54,57}$

\begin{tabular}{llllllll}
\hline P(Efectos actversos) & $\begin{array}{l}\text { PROPESS: } \\
\text { PV } \\
\text { instrumental }\end{array}$ & Cesárea & $\begin{array}{l}P V \\
\text { natural }\end{array}$ & $\begin{array}{l}\text { PREPIDIL } \\
\text { instrumental }\end{array}$ & Cesárea & $\begin{array}{l}P V \\
\text { natural }\end{array}$ \\
\hline Hemorragia post parto & 0,04 & 0,04 & 0,04 & 0,16 & 0,16 & 0,16 \\
Hiperestimulación uterina & 0,04 & 0,04 & 0,04 & 0,03 & 0,03 & 0,03 \\
Náuseas y vómitos & 0,01 & 0,01 & 0,01 & 0,01 & 0,01 & 0,01 \\
Diarrea & 0,01 & 0,01 & 0,01 & 0,01 & 0,01 & 0,01 \\
Sin RAM & 0,88 & 0,88 & 0,88 & 0,77 & 0,77 & 0,77 \\
\hline PV: Parto vaginal. RAM: Reacción Adversa al Medicamento. & & & & & \\
\hline
\end{tabular}

- Igualar la probabilidad de las hemorragias post-parto para ambas alternativas para comprobar su influencia en la decisión final.

- Igualar la tasa de cesáreas al 21,7\% en ambos grupos (recomendada en España por la Agencia de Calidad del SNS en marzo 2006).

- Aumentar el tratamiento de la hiperestimulación uterina con Tractocile ${ }^{\circledR}$ a dos días, es decir aumentando su coste a $275,81 €$ (un bolo y dos perfusiones). Situación que se produce en bastantes ocasiones en la práctica clínica habitual y que no se refleja claramente en los ensayos clínicos.

- Disminuir el coste del procedimiento empleado un $10 \%$ (especialmente de la cesárea).

- Igualar el coste del parto vaginal instrumental al parto natural y que permitirá extrapolar los resultados. Para que se pueda extrapolar nuestros resultados a otros ámbitos sanitarios con menores recursos o más baratos o eficientes.

- Disminuir el coste del Prepidil ${ }^{\circledR} 0,5 \mathrm{mg}$ gel, por utilizar una única dosis endocervical y no repetir la dosis a las seis horas, con un coste por tanto de $15,84 €$.

Debido a que a veces pueden condicionar la decisión al modificar distintas variables conjuntamente, se realizó también un análisis bivariado.
Además se procedió a testar la solidez del modelo utilizado cambiando su estructura, es decir, realizando un análisis de sensibilidad estructural. Se han realizado los siguientes cambios:

- Eliminar la variable de la hemorragias post-parto.

- Incluir en los efectos adversos a los tratamientos. tanto las hemorragias post-parto como la hiperestimulación uterina o hipertono.

Se utilizan en estos modelos de decisión los mismos supuestos ya relatados anteriormente.

\section{RESULTADOS}

Los principales costes de los medicamentos (tanto para la maduración del cérvix e inducción del parto como para el tratamiento de las reacciones adversas) y de los procedimientos empleados, se presentan en la Tabla 3.

Las probabilidades del modelo obtenidas a priori y que sirvieron para diseñar el modelo se presentan en las Tablas 4 y 5.

En la Tabla 5 se presentan las probabilidades empleadas para el calculo de los efectos adversos, mientras que en la Tabla 6 , se resumen los resultados obtenidos con nuestro modelo.

Como se desprende del análisis de estos datos, la op- 
Resultados y discusión

TABLA 6. Resultados de coste efectividad y de ratio coste efectividad incremental de las alternativas estudiadas.

\begin{tabular}{lllllllll}
\hline Estrategia & $\begin{array}{l}\text { Coste } \\
\text { en } \epsilon\end{array}$ & $\begin{array}{l}\text { IC } 95 \% \\
\text { Coste* }\end{array}$ & $\begin{array}{l}\text { Coste } \\
\text { marginal }\end{array}$ & $\begin{array}{l}\text { Efectividad } \\
\text { (n. }{ }^{\circ} \text { partos) }\end{array}$ & $\begin{array}{l}\text { IC 95\% } \\
\text { Efectividad }\end{array}$ & $\begin{array}{l}\text { Efectividad } \\
\text { marginal }\end{array}$ & C/E & RCEI \\
\hline Propess $^{(3)}$ & 2.743 & $2.223-3.542$ & & 1.881 & $0-4.061$ & & 1,46 & Dominada \\
Prepidil $^{(8)}$ & 3.413 & $2.200-6.439$ & 669 & 380 & $0-1.028$ & -1.501 & 8,97 & Dom \\
\hline
\end{tabular}

* IC $95 \%$ calculados con la simulación de Montecarlo con 10.000 reiteraciones para cada alternativa.

ción del Prepidil ${ }^{\circledR} 0,5 \mathrm{mg}$ gel endocervical administrado dos veces al día es dominada por la opción de la administración de una dosis $10 \mathrm{mg}$ de Propess ${ }^{(3)}$ vía vaginal para la maduración del cérvix antes de la inducción del parto en mujeres gestantes nulíparas.

Los resultados del análisis de sensibilidad univariado de todos los parámetros testados muestran la robustez del modelo ya que la decisión de selección del medicamento para la maduración del cérvix se mantiene sin alterarse, tal como se refleja en la Tabla 7 .

Hemos testados todas las posibles combinaciones de las siguientes variables en un análisis de sensibilidad bivariante: cesáreas, parto vaginal natural, parto vaginal instrumental, náuseas y vómitos, diarrea, hiperestimulación uterina, hemorragias post-parto e inducción con oxitocina. En todas las combinaciones el resultado obtenido ha sido de dominancia por parte del Propess ${ }^{\circledR}$ dispositivo vaginal frente a Prepidil ${ }^{\circledR} 0,5 \mathrm{mg}$ gel intracervical. Se comprueba que variando ampliamente todos esos pares de variables, la decisión a tomar sigue siendo la misma en todos los escenarios testados.
Los resultados obtenidos con el análisis de sensibilidad estructural fueron de la misma magnitud que el análisis de sensibilidad uni y bivariado, con una dominancia para la opción de Propess ${ }^{\circledR}$, independientemente de las variables modificadas/añadidas o suprimidas en el modelo.

\section{DIscusión}

La primera decisión al realizar el presente trabajo fue definir cuando y como valorar la efectividad de las medidas adoptadas en las gestantes nulíparas con cuello uterino inmaduro, que acuden al hospital para recibir la correspondiente asistencia sanitaria e inician la inducción al parto con oxitocina. Existen estudios que hacen ambas valoraciones, unos hacen exclusivamente a las 12 horas y otros a las 24 horas. ${ }^{39}$ Durante la realización de este estudio, se decidió emplear el punto de corte más conservador, es decir utilizar solamente la valoración a las 24 horas para la valoración del éxito de las medidas adoptadas, de esta manera ninguna paciente se quedaría sin haber recibido todos los tratamientos necesarios. En

tABLA 7. Análisis de sensibilidad univariado de todos los parámetros testados.

\begin{tabular}{|c|c|c|c|c|c|c|c|}
\hline \multirow[t]{2}{*}{ Parámetro testado } & \multicolumn{3}{|c|}{ PROPESS } & \multicolumn{3}{|c|}{ PREPIDIL } & \multirow[t]{2}{*}{ Decisión } \\
\hline & Coste $(\epsilon)$ & Efectividad & $C / E$ & $\operatorname{Coste}(\epsilon)$ & Efectividad & $C / E$ & \\
\hline $\begin{array}{l}\text { Resultados positivos incluyendo RAM: } \\
\text { diarrea, naúseas... }\end{array}$ & 2.743 & 1.881 & 1,46 & 3.413 & 380,4 & 8,9 & Propess $^{(\otimes)}$ \\
\hline $\begin{array}{l}\text { Solamente incluyendo los partos } \\
\text { sin complicaciones }<24 \text { horas }\end{array}$ & 2.743 & 1.864 & 1,47 & 3.413 & 330,5 & 10,3 & Propess ${ }^{(\mathbb{R}}$ \\
\hline $\begin{array}{l}\text { Efectividad }<24 \mathrm{~h} \text { sin reacciones adversas, } \\
\text { hiperestimulación uterina ni hemorragias }\end{array}$ & 2.743 & 1.859 & 1,48 & 3.413 & 329,6 & 10,3 & Propess $^{(\mathbb{B}}$ \\
\hline $\begin{array}{l}\text { Coste doble de la inducción si no se produce } \\
\text { el parto a las } 24 \text { horas }\end{array}$ & 2.743 & 1.881 & 1,46 & 3.415 & 380,3 & 8,9 & Propess ${ }^{\circledR}$ \\
\hline $\begin{array}{l}\text { Igual la probablidad }(4,8 \%) \text { de hemorragias } \\
\text { post parto }\end{array}$ & 2.743 & 1.881 & 1,46 & 3.047 & 434,7 & 7 & Propess ${ }^{\mathbb{B}}$ \\
\hline $\begin{array}{l}\text { Misma tasa de cesáreas para ambas } \\
\text { estrategias del } 21,7 \%\end{array}$ & 2.740 & 1.885 & 1,45 & 3.356 & 472,1 & 7,1 & Propess $^{\mathbb{B}}$ \\
\hline $\begin{array}{l}\text { Coste mayor con Tractocile }{ }^{\circledR} \text { a } 275,81 € \\
10 \% \text { más barato el coste de la cesárea }\end{array}$ & 2.749 & 1.881 & 1,46 & 3.417 & 380,3 & 8,9 & Propess ${ }^{(}$ \\
\hline $\begin{array}{l}(2.998,64 €) \\
\text { Coste parto instrumental }=\text { natural: }\end{array}$ & 2.668 & 1.881 & 1,42 & 3.292 & 380,3 & 8,6 & Propess ${ }^{\mathbb{B}}$ \\
\hline $2.169,15 €$ & 2.642 & 1.881 & 1,40 & 3.149 & 380,3 & 8,2 & Propess $^{(}$ \\
\hline Dosis única Prepidil ${ }^{\otimes}(15,84 €)$ & 2.743 & 1.881 & 1,46 & 3.397 & 380,3 & 8,9 & Propess ${ }^{\sqrt{(\mathbb{R}}}$ \\
\hline
\end{tabular}


Resultados y discusión

este tipo de gestantes la maduración del cérvix puede conllevar al menos 12 horas de tiempo desde la aplicación del gel o del dispositivo vaginal, más el tiempo necesario para el inicio de efecto de la oxitocina (seis horas como mínimo), y considerando que un tiempo de latencia hasta el parto de 24 horas es un tiempo adecuado para evaluar su eficacia.

Otra asunción realizada en la valoración de la efectividad de los tratamientos fue considerar como exitosos todos los partos por vía vaginal, instrumental o no, sin reacciones adversas independientemente de cuando se produjeran incluso aquellos que se producían transcurridos las 24 horas anteriormente mencionadas. Esto es debido a que los clínicos así lo consideran en la práctica habitual. ${ }^{23,26} \mathrm{Se}$ ha testado en el análisis de sensibilidad univariado esta posibilidad y otras más que la incluyen que no han mostrado cambios en la decisión final seleccionada como muestran los resultados.

En el modelo de decisión diseñado para este estudio hemos incluido todos los eventos adversos especialmente la hiperestimulación uterina ${ }^{39,54}$ y las hemorragias post-parto, si bien anteriores estudios farmacoeconómicos no los incluyeron en el modelo. $45,46,49$

La hiperestimulación uterina o hipertono,${ }^{35}$ es quizás la complicación más frecuente e importante con la utilización de dinosprostona vía endocervical o vaginal, puede aparecer como taquisistolia o hipertono, ambos pueden producir alteraciones en el registro de la fre cuencia cardiaca fetal. La evidencia sugiere que la hiperestimulación uterina después de la administración con dosis más pequeñas de prostaglandina $E_{2}$ es baja, rápidamente reversible con terapia con fármacos $\beta 2$-adrenérgicos (salbutamol o terbutalina), sin que existan complicaciones aparentes maternas o fetales. ${ }^{35}$ En nues tro entorno se emplea para su tratamiento un protocolo con el fármaco atosibán (Tractocile ${ }^{\circledR}$ ), en bolo endovenoso seguido de una perfusión ya comercializada, repitiendo la dosis de la misma hasta su control. Se ha testado ambas posibilidades en el modelo con el mismo resultado de decisión final. Este tratamiento ha demos trado suficiente evidencia favorable a al beneficio/riesgo obtenido respecto los fármacos $\beta 2$-adrenérgicos (salbutamol o terbutalina), a pesar de que éstos tienen un coste muy inferior. ${ }^{44,46}$

Cuando una gestante presenta hipertono el objetivo clínico es controlarlo inicialmente por el riesgo posterior ocasionado para la madre y el feto, por lo que no se toman en cuenta el tiempo de que se produzca el alumbramiento..$^{35}$ Además hemos decidido que un parto vagi nal tras hiperestimulación uterina sin efectos secundarios sea contabilizado como éxito del tratamiento. Este último punto se confirma en el análisis de decisión en la que no se tuvieron en cuenta estos partos para valorar la efectividad de las alternativas comparadas y se obtuvieron resultados similares, por lo que su importancia relativa es baja.

La hemorragias post-parto tras la maduración del cérvix e inducción del parto puede ser mayor o no con la utilización de dinosprostona, pero en ningún momento de- ben desdeñarse por el compromiso vital que supone para la gestante y el aumento del coste que significa por las complicaciones y alargamiento de la estancia hospitalaria que ocasionan. ${ }^{39}$ Estas hemorragias post-parto pueden ser achacables a los tratamientos utilizados para la maduración del cérvix y posterior inducción o no del parto. ${ }^{23} \mathrm{Su}$ importancia radica en la influencia que tiene en la propia calidad de vida de las parturientas y del riesgo que supone para sus vidas, sin omitir la cantidad de recursos empleados en su resolución: transfusiones, mantenimiento del volumen plasmático, oxigenoterapia, controles laboratorio, etc. ${ }^{23}$ Es obligado tener en cuenta esta variable, y por ello se ha decidido emplearla como una variable independiente del resto de los eventos secundarios a los tratamientos para valorar su peso en la toma de decisiones. El modelo en el que se suprime esta variable en el análisis de sensibilidad no muestra diferencias en la toma de decisiones, ni tampoco al igualar el porcentaje de hemorragias post-parto en ambos tratamientos.

Otros efectos secundarios de la aplicación local de dinoprostona son las alteraciones gastrointestinales, la diarrea y las náuseas y vómitos, que no siempre se recogen en los estudios publicados por su menor frecuencia de aparición al emplearse el fármaco localmente. $41,42,45$ Sin embargo en nuestro estudio estos efectos adversos más leves, también fueron incluidos en el modelo, aunque su importancia tanto en costes como en su prevalencia es poco relevante. Se ha testado la asignación como partos con éxito cuando las pacientes presentaban estos efectos adversos en ambos grupos de tratamiento no mostrándose diferencias en el fármaco seleccionado como opción más favorable.

En el presente trabajo no se ha tenido en cuenta en el modelo de decisión la mortalidad de las gestantes ni de los recién nacidos. Afortunadamente es muy baja su prevalencia. ${ }^{23,27} \mathrm{Su}$ registro diferenciado, es decir de mujeres nulíparas con el cérvix inmaduro que serian la población diana de nuestro estudio, del total de mortalidad infantil y de la mortalidad de las gestantes no ha sido posible obtenerla, seguramente porque se necesiten series de pacientes muy grandes para poder registrarlas debido a su muy baja incidencia. ${ }^{23,26}$

Existen alternativas farmacológicas a la dinoprostona, recientemente se ha comercializado el misoprostol (análogo sintético de la prostaglandina $\mathrm{E}_{1}$ con resultados prometedores) como comprimidos vaginales (Misofar ${ }^{(}$, a dosis de 25 y $200 \mathrm{mcg}$ ). ${ }^{30-32}$ Existen suficientes evidencias para su utilización exitosa en la maduración del cérvix e incluso hay estudios que lo recomiendan por tener mejor relación coste efectividad que la prostaglandina $\mathrm{E}_{2} \cdot{ }^{30}$ Además este fármaco por su capacidad de provocar a dosis altas la dilatación del útero no grávido es utilizado también para la interrupción del parto, pudiéndose en ocasiones omitir o confundir ambas indicaciones en los hospitales. Es por todo ello por lo que no lo incluimos en el modelo inicial. En un próximo estudio, al tener ya validado el modelo de decisión, se podría comparar las formas tópicas de dinoprostona con los comprimidos vaginales de misoprostol, para que lle- 
Resultados y discusión

gásemos a la decisión óptima para el tratamiento de estas mujeres gestantes.

Gracias a los resultados publicados por Taylor y Armour ${ }^{49}$ sobre las preferencias reveladas por mujeres gestantes australianas mediante su disponibilidad a pagar sobre todos los aspectos relacionados con el tratamiento con gel endocervical de dinoprostona o con oxitocina y ruptura artificial de membranas, se abre la posibilidad de realizar estudios coste-utilidad en esta indicación. El modelo de decisión presentado en este trabajo puede perfectamente utilizarse para realizar estudios de costeutilidad ya que en la práctica asistencial cada día se tienen más en cuenta las opiniones de los pacientes acerca de su tratamiento.

En este estudio además de variar los valores de los parámetros del modelo, es posible cambiar la estructura propiamente dicha del modelo de decisión utilizado. Esta técnica permite la posibilidad de comparar nuestro modelo con otros ya existentes. Normalmente en medicina no se realiza el análisis de sensibilidad estructural, en nuestra opinión, por varias razones:

- procedimiento mucho más laborioso;

- la reducción de la incertidumbre no es realmente muy alta, se trata más bien de una medida complementaria más;

- requiere de conocimientos profundos en la aplicación de la metodología;

- complejidad alta de realizar en el mundo sanitario, ya que las cosas suelen ocurrir de manera consecutiva y se toman muchas decisiones en un corto espacio de tiempo; y,

-... su validez clínica debe ser contrastada en cada una de las modificaciones realizadas.

El tratamiento de las gestantes a término nulíparas con el cuello uterino inmaduro con una dosis del medicamento Propess $^{\text {B }} 10 \mathrm{mg}$ sistema de liberación vaginal (dinoprostona) presenta una relación más favorable coste efectiva que el tratamiento con dos dosis separadas seis horas entre sí de Prepidi1 ${ }^{\circledR} 0,5 \mathrm{mg}$ gel endocervical $(2,5 \mathrm{ml})$, que contiene también la prostaglandina $E_{2}$ como principio activo.

Otras posibles conclusiones derivadas del estudio serían:

- Robustez del modelo de decisión utilizado en los escenarios testados.

- La modificación de la estructura del árbol de decisión aporta información y da mayor validez al modelo utilizado.

- Poca importancia tienen los efectos gastrointestinales leves para evaluar la aplicación local de dinoprotona.

- La hiperestimulación uterina es un efecto adverso que debe ser siempre incluido en los modelos que valoren los fármacos análogos de la prostaglandina $\mathrm{E}_{2}$. Asimismo deben contemplarse las hemorragias post-parto que se produzcan.
- En el futuro, será conveniente la realización de estudios que incluyan datos de supervivencia de la gestante y del feto para completar todos los eventos posibles.

- Es posible utilizar el modelo desarrollado en otros tipos de estudios como los de coste-utilidad. $\mathrm{AF}$

\section{BIBLIOGRAFÍA}

1. Kelly AJ, Tan B. Intravenous oxytocin alone for cervical ripening and induction of labour. Cochrane Database of Systematic Reviews 2001; 3: CD003246.

2. Capogna G, Parpaglioni R, Lyons G, Columb M, Celleno D. Minimum analgesic dose of epidural sufentanil for first-stage labor analgesia: a comparison between spontaneous and prostaglandininduced labors in nulliparous women. Anesthesiology 2001; 94 : induce-4.

3. Chen LK, Hsu HW, Lin CJ, Huang CH, Tsai SK, Lee CN, et al. Effects of epidural fentanyl on labor pain during the early period of the first stage of induced labor in nulliparous women. J Formos Med Assoc 2000; 99: 549-53.

4. Balladur A, Tribalat S, Jaouen E, Tochon C, Lewin D. When should epidural analgesia be started in cases of induction of labour? Results of a randomised prospective study. J Gynecol Obstet Biol Reprod (París) 1989; 18: 249-54.

5. Chestnut DH, Vincent Jr RD, McGrath JM, Choi WW, Bates JN Does early administration of epidural analgesia affect obstetric outcome in nulliparous women who are receiving intravenous oxytocin? Anesthesiology 1994; 80: 1193-200.

6. The Information Centre CHS. NHS Maternity Statistics, England: 2004-5. Leeds: The Information Centre; 2006.

7. Grubb DK, Rabello YA, Paul RH. Post-term pregnancy: fetal death rate with antepartum surveillance. Obstet Gynecol 1992; 79 ; $1024-6$

8. Kozak LJ, JD Weeks. US trends in obstetric procedures, 1999 2000. Birth 2002; 29: 157-61.

9. Maslow AS, Sweeney AL. Elective induction of labor as a risk factor for cesarean delivery among low-risk women at term. Obstet Gynecol 2000; 95 (6 Pt 1): 917-22.

10. Danforth DN, Veis A, Breen M, Weinstein HG, Buckingham JC Manalo $P$. The effect of pregnancy and labor on the human cervix, changes in collagen, glycoproteins and glycosaminoglycans. Am J Obstet Gynecol 1974: 120: 641-51.

11. Department of Health. Changing Childbirth: Part 2 Survey of Good Communications Practice in Maternity Services. London: HMSO; 1993.

12. Bishop EH. Pelvic scoring for elective induction. Obstet Gynecol 1964; 24: 266-8.

13. Alexander JM, McIntire DD, Leveno KJ. Forty weeks and be yond: pregnancy outcomes by week of gestation. Obstet Gyneco 2000; 96: 291-4.

14. Feldman GB. Prospective risk of stillbirth. Obstet Gynecol 1992; 79: $547-53$.

15. Hilder L, Costeloe $\mathrm{K}$, Thilaganathan B. Prolonged pregnancy: evaluating gestation-specific risks of fetal and infant mortality. Br J Obstet Gynaecol 1998; 105: 169-73.

16. Cotzias CS, Paterson-Brown S, Fisk NM. Prospective risk of unexplained stillbirth in singleton pregnancies at term: population based analysis. BMJ 1999; 319: 287-8.

17. Treger M, Hallak M, Silberstein T, Friger M, Katz M, Mazor M. Post-term pregnancy: should induction of labor be considered before 42 weeks? J Matern Fetal Neonatal Med 2002; 11: 50-3.

18. Caughey AB, Bishop JT. Maternal complications of pregnancy increase beyond 40 weeks of gestation in low-risk women. $\mathrm{J}$ Perinatol 2006; 26: 540-5. 
Resultados y discusión

19. Heimstad R, Romundstad PR, Eik-Nes SH, Salvesen KA. Outcomes of pregnancy beyond 37 weeks of gestation. Obstet Gynecol 2006; 108 (3 Pt 1): 500-8

20. Olesen AW, Westergaard JG, Olsen J. Perinatal and materna complications related to postterm delivery: a national registerbased study, 1978-1993. Am J Obstet Gynecol 2003; 189: 222-7.

21. Smith GC. Life-table analysis of the risk of perinatal death a term and post term in singleton pregnancies. Am J Obstet Gynecol 2001; 184: 489-96.

22. Bakketeig LS, Bergsio P. Post-Term pregnancy: Magnitude of the problem. In Effective Care in Pregnancy and Childbirth. M Enkin, MJ Keirse, I Chalmers. Oxford, Eds. Oxford University Press, 1989: 772-85.

23. Protocolos asistenciales en Obstetricia. Embarazo cronológicamente prolongado. Protocolo de la S.E.G.O. Madrid. 2004

24. Shea KM, Wilcox AJ, Little RE. Postterm delivery: a challenge for epidemiologic research. Epidemiology 1998; 9: 199-204.

25. Gülmezoglu AM, Crowther CA, Middleton P. Induction of labour for improving birth outcomes for women at or beyond term. Cochrane Database of Systematic Reviews 2006; 4: CD004945.

26. National Collaborating Centre for Women's and Children's Health. Antenatal Care: Routine Care for the Healthy Pregnant Woman. 2nd edition. London: RCOG Press; 2008.

27. Lange AP. Induction of labour. Dan Med Bull 1984; 31: 89-108.

28. Chua S, Arulkumaran S, Vanaja K, Ratnam SS. Preinduction cervical ripening: prostaglandin $\mathrm{E}_{2}$ gel $v s$ hygroscopic mechanical dilator. J Obstet Gynaecol Res 1997; 23:171-7.

29. Yapar EG, Senöz S, Urkütür M, Batioglu S, Gökmen O. Second trimester pregnancy termination including fetal death: comparison of five different methods. Eur J Obstet Gynecol Reprod Biol 1996; 69: 97-102.

30. Wing DA, Rahall A, Jones MM, Goodwin TM, Paul RH. Misoprostol: an effective agent for cervical ripening and labor induction. Am J Obstet Gynecol 1995: 172: 1811-6.

31. Chuck FJ, Huffaker BJ. Labor induction with intravaginal misoprostol versus intracervical prostaglandin $\mathrm{E}_{2}$ gel (Prepidil gel) randomized comparison. Am J Obstet Gynecol 1995; 173: 1137-42

32. Magtibay PM, Ramin KD, Harris DY, Ramsey PS, Ogburn PL Jr. Misoprostol as a labor induction agent. J Matern Fetal Med 1998; 7: $15-8$

33. Chyu JK, Strassner HT. Prostaglandin $E_{2}$ for cervical ripening: a randomized comparison of Cervidil versus Prepidil. Am J Obstet Gynecol 1997; 177: 606-11.

34. Dalui R, Suri V, Ray P, Gupta I. Comparison of extraamniotic Foley catheter and intracervical prostaglandin $\mathrm{E}$ gel for preinduction cervical ripening. Acta Obstet Gynecol Scand 2005; 84: 362-7.

35. Egarter CH, Husslein PW, Rayburn WF. Uterine hyperstimulation after low-dose prostaglandin E2 therapy: tocolytic treatment in 181 cases. Am J Obstet Gynecol 1990; 163: 794-6.

36. Facchinetti F, Venturini P, Fazzio M, Volpe A. Elective cervical ripening in women beyond the 290th day of pregnancy: a randomized trial comparing 2 dinoprostone preparations. J Reprod Med 2007; 52: 945-9.

37. Kelly AJ, Kavanagh J, Thomas J. Vaginal prostaglandin (PGE2 and PGF2a) for induction of labour at term. Cochrane Database of Systematic Reviews 2003; 4: CD003101

38. Kemp B, Winkler M, Rath W. Induction of labor by prostaglandin $\mathrm{E}(2)$ in relation to the Bishop score. Int J Gynaecol Obstet 2000; 71: 13-7.

39. Hughes EK, Kelly AJ, Kavanagh J: Dinoprostone vaginal inser for cervical ripening and labor induction: A meta-analysis. Obstet Gynecol 2001; 97: 847-55.

40. Rayburn WF. Clinical experience with a controlled-release, prostaglandin $\mathrm{E}_{2}$ intravaginal insert in the USA. Br J Obstet Gynaeco 1997; 104 Suppl 15: 8-25.
41. Prepidil ${ }^{2} 0,5 \mathrm{mg}$ gel endocervical. Ficha técnica. Pfizer S.A. Disponible en: https://sinaem4.agemed.es/consaem/especialidad.do ?metodo $=$ verFichaWordPdf\&codigo $=57694 \&$ formato $=$ pdf $\&$ formulario=FICHAS (Consulta: 15 septiembre 2010).

42. Propessio $10 \mathrm{mg}$ sistema de liberación vaginal. Ficha técnica. Ferring S.A.U. Disponible en:https://sinaem4 agemed.es/consaem/ especialidad.do? metodo $=$ verFichaWordPdf $\&$ codigo $=62088 \&$ for mato=pdf\&formulario=FICHAS (Consulta: 15 septiembre 2010).

43. Sanchez Ramos L, Olivier F, Delke I, Kaunitz AM. Labor induc tion versus expectant management for postterm pregnancies: a systematic review with meta-analysis. Obstet Gynecol 2003; 101: 1312-8.

44. Davies LM, Drummond MF. Management of labour: consume choice and cost implications. J Obstet Gynaecol 1991; 11 (Suppl 1): S28-S33.

45. Hass SL, Lucas MJ. Net economic benefit of a manufactured dinoprostone gel for pre-induction cervical ripening. Pharmacoeconomics 1994: 5: 39-47.

46. Taylor SJ, Armour CL. Cost implications in the management of induction of labour. Pharmacoeconomics 1997; 12: 547-54.

47. Wieland D, Friedman F Jr. Comparing two dinoprostone agent for preinduction cervical ripening at term. A randomized trial. J Reprod Med 1999; 44: 724-8.

48. Stewart JD, Rayburn WF, Farmer KC, Liles EM, Schipul AH Ji Stanley JR. Effectiveness of prostaglandin $E_{2}$ intracervical gel (Prepidil $^{\mathbb{R}}$ ), with immediate oxytocin, versus vaginal insert (Cervidil $\left.{ }^{*}\right)$ for induction of labor. Am J Obstet Gynecol 1998; 179 ; 1175-80.

49. Taylor S, Armour C. Consumer preference for dinoprostone vagi nal gel using stated preference discrete choice modelling. Pharmacoeconomics 2003; 21: 721-35.

50. Hannah ME, Hannah WJ, Hellman J, Hewson S, Milner R, Willan A. Induction of labor as compared with serial antenata monitoring in post-term pregnancy: A randomized control trial. N Engl J Med 1992; 326: 1587-92.

51. Marconi AM, Bozzetti P, Morabito A, Pardi G. Comparing two dinoprostone agents for cervical ripening and induction of labor: a randomized trial. Eur J Obstet Gynecol Reprod Biol 2008; 138 : $135-40$.

52. Rath W. A clinical evaluation of controlled-release dinoprostone for cervical ripening-a review of current evidence in hospital and outpatient settings. J Perinat Med 2005; 33; 491-9.

53. Ramsey PS, Harris DY, Ogburn PL Jr, Heise RH, Magtibay PM, Ramin KD. Comparative efficacy and cost of the prostaglandin analogs dinoprostone and misoprostol as labor preinduction agents. Am J Obstet Gynecol 2003; 188: 560-5.

54. Sanchez-Ramos L, Farah LA, Kaunitz AM, Adair CD, Del Valle GO, Fuqua P. Preinduction cervical ripening with commercially available prostaglandin $\mathrm{E} 2$ gel: a randomized, double-blind comparison with a hospital-compounded preparation. Am J Obstet Gynecol 1995;173: 1079-84.

55. Nicholson JM, Kellar LC, Cronholm PF, Macones GA. Active management of risk in pregnancy at term in an urban population An association between a higher induction of An association between a highe ind 1516-28.

56. Sanchez-Ramos L, Kaunitz AM, Connor PM. Hygroscopic cervical dilators and prostaglandin E2 gel for preinduction cervical ripening. A randomized, prospective comparison. J Reprod Med 1992; 37: 355-9.

57. Sanchez Ramos L, Kaunitz AM, Delke I, Gaudier FL. Cervical ripening and labor induction with a controlled-release dinoprostone vaginal insert. A meta-analysis. Obstet Gynecol 1999; 94 $878-83$. 


\section{Capítulo 7}

Carrera-Hueso FJ, Ramón Barrios A. Análisis de sensibilidad estructural. Farm Hosp 2011; 35 (Supl 1): 10-17. 


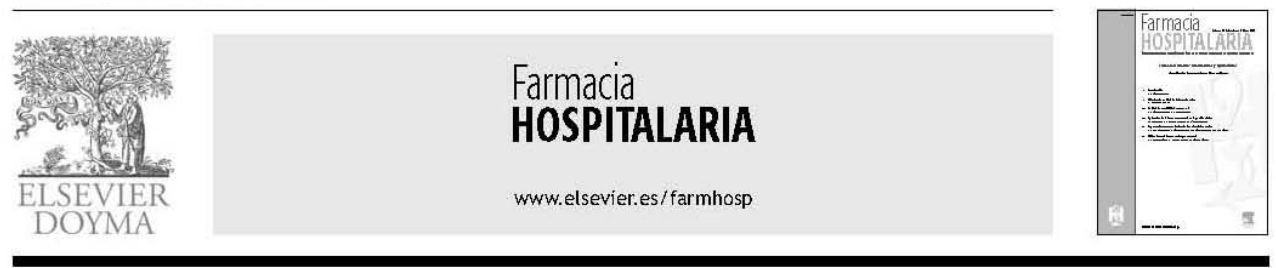

\section{Análisis de sensibilidad estructural}

\section{F.J. Carrera-Hueso* y A. Ramón-Barrios}

Servicio de Farmacia, Hospital Dr. Moliner, Serra, Valencia, España

PALABRAS CLAVE

Árbol de decisión

Análisis de

sensibilidad

estructural;

Farmacoeconomía;

Modelización;

Diagrama de

influencia;

Coste-efectividad

\section{KEYWORDS}

Decision tree;

Structural sensitivity

analysis;

Pharmacoeconomics;

Modelling;

Influence diagram;

Cost-effectiveness
Resumen El objetivo fue realizar un análisis de sensibilidad estructural a un modelo de decisión y extraer sus ventajas y limitaciones. Se tomó como modelo base uno previamente publicado sobre dinoprostona que se modificó teniendo en cuenta dos escenarios: eliminando las hemorragias posparto e incluyendo en los efectos adversos tanto las hemorragias como la hiperestimulación uterina. El resultado del anál isis de sensibilidad estructural realizado muestra la robustez del modelo base y confirma los resultados iniciales: el dispositivo intrauterino de dinoprostona es más coste-efectivo que administrado en gel endocervical. El análisis de sensibilidad estructural debe ser congruente con la situación estudiada y validado clínicamente. Aunque la reducción de la incertidumbre obtenida puede no ser alta, sí aporta información y proporciona mayor validez y fiabilidad al modelo.

(c) 2011 SEFH. Publicado por Elsevier España, S.L. Todos los derechos reservados.

Structural sensitivity analysis

Abstract The aim of this study was to perform a structural sensitivity analysis of a decision model and to identify its advantages and limitations. A previously published model of dinoprostone was modified, taking two scenarios into account: eliminating postpartum hemorrhages and including both hemorrhages and uterine hyperstimulation among the adverse effects. The result of the structural sensitivity analysis shows the robustness of the underlying model and confirmed the initial results: the intrauterine device is more cost-effective than intracervical dinoprostone gel. Structural sensitivity analyses should be congruent with the situation studied and clinically validated. Although uncertainty may be only slightly reduced, these analyses provide information and add greater validity and reliability to the model. (c) 2011 SEFH. Published by Elsevier España, S.L. All rights reserved.

Autor para correspondencia.

Correo electrónico: javier_carrera2690@yahoo.es (F.J. Carrera-Hueso).

1130-6343/\$ - see front matter $\mathbb{2} 2011$ SEFH. Publicado por Elsevier España, S.L. Todos los derechos reservados. 
Resultados y discusión

\section{Introducción}

El análisis de decisión es una técnica cuantitativa que en su aplicación al sector sanitario pretende ayudar a resolver problemas, considerando todas las alternativas y todos los sucesos de interés, incluyendo las probabilidades de los acontecimientos. Los modelos de decisión se emplean con el fin de obtener información del mundo real. En efecto, un modelo es una representación estructurada de los acontecimientos del mundo real o una aproximación sistemática a las condiciones reales, diseñado con el fin de evaluar el impacto de las estrategias médicas sobre los costes generados o los resultados obtenidos, bajo condiciones de incertidumbre $^{1.3}$. La modelización puede realizarse mediante árboles de decisión, diagramas de influencia, modelos de Markovo mediante modelos de eventos discretos ${ }^{2,3}$

La utilización de modelos económicos en farmacoecono. mía ha sido exponencial en los últimos años debido a las grandes ventajas que presenta frente a la realización de es. tudios diseñados expresamente, bien incluidos en ensayos clínicos o realizando estudios naturalísticos ${ }^{46}$. En efecto, la menor cantidad de recursos necesarios para llevar a cabo un modelo es su principal ventaja frente a las opciones anteriormente mencionadas, pero además tampoco están exentos de sesgos este tipo de diseños más pragmáticos, es decir, los estudios naturalísticos no son la panacea de los estudios farmacoeconómicos ${ }^{4,6}$.

La incertidumbre sobre los costes, los beneficios y las relaciones entre ambos puede tener un doble origen. Por un lado, puede existir incertidumbre acerca de los parámetros. Esto ocurre cuando no puede conocerse con certeza cuáles son los verdaderos valores numéricos de tales parámetros. Por otra parte, podemos enfrentarnos a la incertidumbre sobre el modelo, esto es, incertidumbre acerca de cuál es la forma óptima de combinar estos parámetros ${ }^{6}$.

La incertidumbre sobre el valor verdadero de los parámetros puede tener varias causas ${ }^{3}$ : a) en primer lugar, determinados parámetros no pueden ser observados; un ejemplo es la efectividad a largo plazo en algunos trata. mientos, cuyo conocimiento exigiría que la duración del estudio fuese muy larga, y raramente se sigue a los pacientes de forma prolongada en el tiempo; b) una segunda causa de incertidumbre se deriva de la ausencia de consenso teórico acerca del valor más apropiado para un parámetro; por ejemplo, hay opiniones muy diversas sobre la forma de estimar el coste de oportunidad del tiempo perdido por pacientes o familiares que no están trabajando; c) en tercer lugar, puede existir incertidumbre en relación con aspectos básicos del tratamiento; por ejemplo, puede que la epidemiología de la enfermedad, la conducta del médico o la adherencia al tratamiento sean poco conocidas; y d) otra fuente de incertidumbre son las posibles divergencias entre la población de la cual se han obtenido los valores de los parámetros y la población en la cual se quiere estimar el coste-efectividad.

Como se puede ver, la incertidumbre que afecta a los parámetros puede deberse a muy diversas causas y ser originada por diferentes fuentes. Están sujetos a incertidumbre los datos procedentes de ensayos clínicos, los estimados mediante el metaanálisis de la literatura, los sugeridos por expertos, los estimados mediante modelización, etc. Prácticamente, cualquier parámetro incluido en el análisis es susceptible de provocar incertidumbre sobre el resultado de una evaluación económica ${ }^{2-6}$.

Dado que es imposible eliminar la incertidumbre, el obje tivo ha de ser su incorporación al estudio. El método más habitual para tratar el problema de la incertidumbre acerca de los parámetros es el análisis de sensibilidad $30,5,6$.

El análisis de sensibilidad tradicional es un método determinístico - por oposición a los métodos probabilísticos- que trata de aislar los efectos de la variación de los valores de los distintos parámetros para que el analista pueda observar la influencia que dicha variación tiene sobre el resultado fi. nal del estudio?.

La forma tradicional de análisis de sensibilidad es el análisis univariado. El modo de proceder consiste en modificar el valor de un único parámetro y observar cómo cambian los resultados. A continuación, el procedimiento se repite con cada uno de los parámetros sujetos a incertidumbre -uno sólo cada vez-, manteniendo el resto de parámetros constantes. El análisis de sensibilidad univariado permite identificar los parámetros que resultan más influyentes en los resultados finales y sirve para valorar e grado de robustez del análisis. Sin embargo, y pese a ser el más usado, este tipo de análisis no es teóricamente el más correcto. La razón principal estriba en que en la realidad los parámetros no son independientes 0 , incluso aunque lo sean, no suelen variar de forma aislada. Otra razón por la cual el análisis univariado no es la mejor opción desde un punto de vista teórico radica en el hecho de que lo que interesa en una evaluación económica es la relación entre los costes y los beneficios, normalmente expresada mediante un cociente -la ratio coste-efectividad-, y la variabilidad de dicho cociente es mayor que la variabilidad independiente del numerador y el denominador por separado ${ }^{7-9}$.

Existen varias alternativas al análisis univariado a la hora de tratar la incertidumbre. En efecto, disponemos del análisis multivariado, el análisis de escenarios extre mos, el análisis de umbral, y por último, el análisis de sensibilidad probabilístico. No explicaremos aquí estas técnicas al tener limitaciones editoriales y no ser el obje to del trabajo, pero se pueden consultar en múltiples textos de referencia $2,3,7 \cdot 9$.

La incertidumbre puede afectar, no ya a los valores de los parámetros utilizados en el análisis, sino a la forma funcional del modelo que combina tales parámetros. Cuando esto ocurre, es decir, cuando no se tiene certeza sobre la forma matemática en que se combinan determinados parámetros, no existen métodos claros para proceder a su tratamiento e incorporación al análisis. En cualquier caso, lo que sí se ha de hacer es reconocer en el informe esta incertidumbre, así como explicitar la opción metodológica elegida ${ }^{4,6}$.

En realidad, una de las pocas sugerencias que se puede hacer al respecto consiste en llevar a cabo un análisis de sensibilidad de los modelos. Es decir, se podrían calcular las ratios coste-efectividad bajo los dos modelos alternativos y comprobar la magnitud del cambios.

En el campo médico no se realiza habitualmente un análisis de sensibilidad estructural de los modelos utilizados de bido a múltiples factores, sobre todo a su alta complejidad y coste de recursos. En una revisión de las guías publicadas solamente 4 de las 16 seleccionadas como excelentes reco- 
Resultados y discusión

mendaban un análisis estructural del modelo, aunque la mayoría de ellas indicaban que se deben justificar las asunciones consideradas en su elaboración y, además, la relación entre las variables incluidas en el model $0^{6,10 \cdot 13}$.

El objetivo de este trabajo es realizar un análisis de sensibilidad estructural a un modelo de decisión propuesto como ejemplo y extraer las ventajas y limitaciones de su aplicación.

\section{Método}

Se tomó como caso base un trabajo publicado recientemente cuyo objetivo fue determinar cuál de las dos presentaciones de dinoprostona, gel endocervical y dispositivo intrauterino, a dosis usuales, presentaba mejor relación coste-efectividad para la maduración del cérvix antes de la inducción del parto ${ }^{14}$. Las alternativas comparadas fueron dos presentaciones de dinoprostona, dos dosis separadas en tre seis horas de gel endocervical (Prepidil ${ }^{\circ}$ ), y una dosis única del dispositivo intrauterino (Propess $^{\natural}$ ) presentaba mejor relación coste-efectividad para la maduración del cérvix antes de la inducción del parto. Para ello, se diseñó un árbol de decisión que recogió los efectos de los fármacos y sus eventos adversos, siendo la perspectiva utilizada la del hospital y el horizonte temporal contemplado menor a un año. Las fuentes de efectividad y costes, así como las probabilidades de los eventos, están descritas en el trabajo de referencia" ${ }^{14}$.

Los programas informáticos utilizados en el diseño, desarrollo y resolución de los modelos propuestos para el estudio coste-efectividad han sido: Microsoft Office Excel 2003, Data 3.5 (TreeAge Software 1998) ${ }^{15}$ y Elvira versión $0.162^{16}$. Se realizó el diagrama de ínfluencia inicial del modelo con el programa Elvira, introduciendo todas las probabilidades de los acontecimientos, y se resolvió mediante un árbol de decisión mediante el programa Data 3.5, el cual puede consultarse en el trabajo original ${ }^{14}$. El programa MS Excel ha servido de apoyo para comprobar todo el modelo.

Se separaron como resultados obtenidos los partos como cesáreas, vaginales, bien naturales o instrumentales. Se asumió que un parto instrumental conllevaba un día más de hospitalización frente al parto vaginal natural. Los principales eventos adversos de la utilización de dinoprostona localmente se recogen como variables independientes (hemorragias posparto e hiperestimulación uterina). Se esti. mó que en todas las pacientes que presenten hiperestimulación o hipertono se proceda directamente a su manejo instauración de las maniobras del parto, sin necesidad de esperar las $\mathbf{2 4}$ horas para valorar su efectividad. En el mode lo se asume que para la maduración del cérvix es necesario el transcurso de 24 horas del inicio del tratamiento con prostaglandina $E_{2}$ para valorar su eficacia.

$\mathrm{El}$ análisis de sensibilidad estructural se realizó partiendo del modelo base disponible con el diagrama de influencia que es más flexible y sufre menos variaciones que un árbol de decisión ${ }^{17,18}$. Se modificó teniendo en cuenta los dos supuestos contemplados, y fueron: a) eliminar la variable de la hemorragias posparto (modelo 1); $y$ b) incluir en los efectos adversos a los tratamientos, tanto las hemorragias posparto como la hiperestimulación uterina o hipertono (modelo 2).
Cada modelo fue resuelto mediante su árbol de decisión correspondiente ${ }^{18}$. En estos modelos alternativos también se realizó el análisis de sensibilidad, variando los parámetros con los mismos escenarios y supuestos ya relatados anteriormente en el modelo base.

\section{Resultados}

Los principales resultados obtenidos con el modelo base se muestran en la tabla 1, y el resto de resultados obtenidos con el análisis de sensibilidad puede consultarse en la referencia original ${ }^{14}$; además, en la figura 1 se muestra el diagrama de influencia de la relación entre las variables implicadas obtenido con el programa Elvira ${ }^{16}$.

Con el modelo se obtuvieron 108 posibles resultados finales para cada una de las alternativas estudiadas. El coste medio por cada parto vaginal sin complicaciones ni efectos adversos fue de $1,46 €$ para el Propess ${ }^{\oplus}$ y de $8,97 €$ para el grupo tratado con Prepidil ${ }^{\circ}$ gel. La utilización del dispositivo vaginal supone un ahorro por paciente de $669,6 €$ para el hospital. Como se desprende del análisis de estos datos, la opción del Prepidil ${ }^{\oplus} 0,5 \mathrm{mg}$ gel endocervical, administrado dos veces al día, es dominada por la opción de la adminis. tración de una dosis $10 \mathrm{mg}$ de Propess ${ }^{\oplus}$ vía vaginal para la maduración del cérvix antes de la inducción del parto en mujeres gestantes nulíparas. Los resultados del análisis de sensibilidad univariado de todos los parámetros testados muestran la robustez del modelo, ya que la decisión de selección del medicamento para la maduración del cérvix se mantiene sin alterarse.

Al eliminar como variable independiente las hemorragias posparto del modelo base e incluirla en la de efectos adversos se obtiene el modelo 1 del análisis de sensibilidad es. tructural, obteniendo el gráfico de la figura 2.

En la figura 3 se muestra de manera abreviada la resolución del modelo 1 planteado mediante su correspondiente árbol de decisión. La resolución de este modelo se sintetiza en la tabla 2. Por lo tanto, la elección de una presentación de dinoprostona para la maduración del cérvix en mujeres gestantes a término no varía del modelo matriz o madre de que partíamos. La ratio coste-efectividad incremental fue de $0,20 €$, lo que supone un ahorro de 20 céntimos por cada parto sin complicaciones al utilizar Propess ${ }^{\oplus}$ en vez de Prepidil $^{\star}$ gel.

Al considerar todos los efectos adversos de los medicamentos en el mismo nodo que representa esa variable, es decir, incluyendo la hiperestimulación uterina y la hemorragia posparto, nos quedaría el siguiente diagrama de influencia, el modelo 2 representado en la figura 4 . Las probabilidades a priori del nodo de efectos adversos del modelo 2 se muestran en la tabla 3 , y sus principales resultados se detallan en la tabla 4. Por lo tanto, con este modelo obtenemos el mismo resultado, es decir, la decisión óptima en mujeres gestantes nulíparas y que presenten el cuello uterino inmaduro sigue siendo la utilización de una dosis de Propess $10 \mathrm{mg}$ vía vaginal para la maduración del cérvix antes de la posible inducción o no del parto. La ratio coste-efectividad incremental obtenido fue de $-0,45$, similar al modelo base, lo que nos da una idea de la similitud del modelo utilizado con el modelo de referencia, al cambiar su estructura. 
Resultados y discusión

El resultado del análisis de sensibilidad estructural realizado muestra la robustez del modelo base y confirma los resultados obtenidos inicialmente, es decir, que la dinoprostona aplicada mediante el dispositivo intra-

terino posee una relación coste-efectiva más favorable que dos aplicaciones de la dinoprostona en gel endocervical; utilizando dosis convencionales en ambas presentaciones.

Tabla 1 Resultados del análisis coste-efectividad del modelo base

\begin{tabular}{lccccccccc}
\hline \multicolumn{1}{c}{ Estrategia } & $\begin{array}{c}\text { Coste } \\
\text { en } €\end{array}$ & $\begin{array}{c}\text { IC95\% } \\
\text { Coste }^{*}\end{array}$ & $\begin{array}{c}\text { Coste } \\
\text { marginal }\end{array}$ & $\begin{array}{c}\text { Efectividad } \\
\text { (n. partos) }\end{array}$ & $\begin{array}{c}\text { IC95\% } \\
\text { Efect..* }\end{array}$ & $\begin{array}{c}\text { Efectividad } \\
\text { marginal }\end{array}$ & C/E & RCEI & \\
\hline Dispositivo intrauterino & $2.743,5$ & $2.223,3-3.542,93$ & & $1.881,4$ & $0-4.061,07$ & & 1,46 & \\
Gel endocervical & $3.413,1$ & $2.200,83-6.439,30$ & 669,6 & 380,3 & $0-1.028,74$ & $-1.501,0$ & 8,97 & Dominada \\
\hline
\end{tabular}

IC95\% calculados con la simulación de Montecarlo con 10.000 reiteraciones para cada alternativa; RCEl: ratio coste-efectividad incremental.

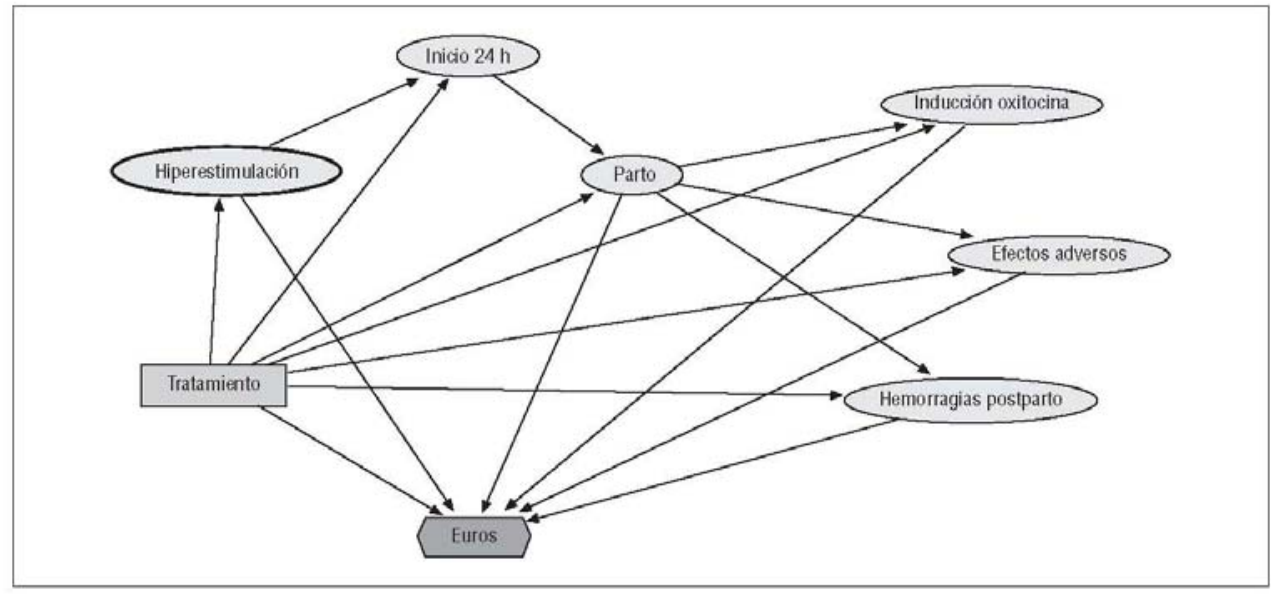

Figura 1 Diagrama de influencía del modelo base.

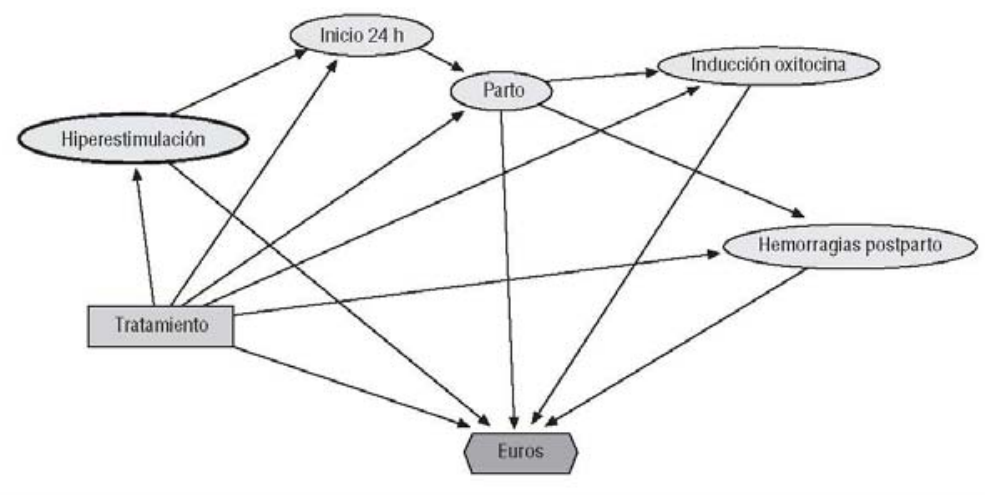

Figura 2 Diagrama de influencia del modelo 1. 
Resultados y discusión

F.J. Carrera-Hueso y A. Ramón-Barrios

\begin{tabular}{|c|c|c|c|c|c|c|}
\hline Estrategia & $\begin{array}{l}\text { Coste } \\
\text { en } €\end{array}$ & $\begin{array}{c}\text { Coste } \\
\text { marginal }\end{array}$ & $\begin{array}{l}\text { Efectividad } \\
\text { (n. partos) }\end{array}$ & $\begin{array}{c}\text { Efectividad } \\
\text { marginal }\end{array}$ & C/E & RCEI \\
\hline Dispositivo intrauterino & $2.595,8$ & & $2.075,9$ & & 1,25 & \\
\hline Gel endocervical & $2.899,4$ & 303,6 & 548,1 & $-1.527,8$ & 5,29 & Dominada \\
\hline
\end{tabular}

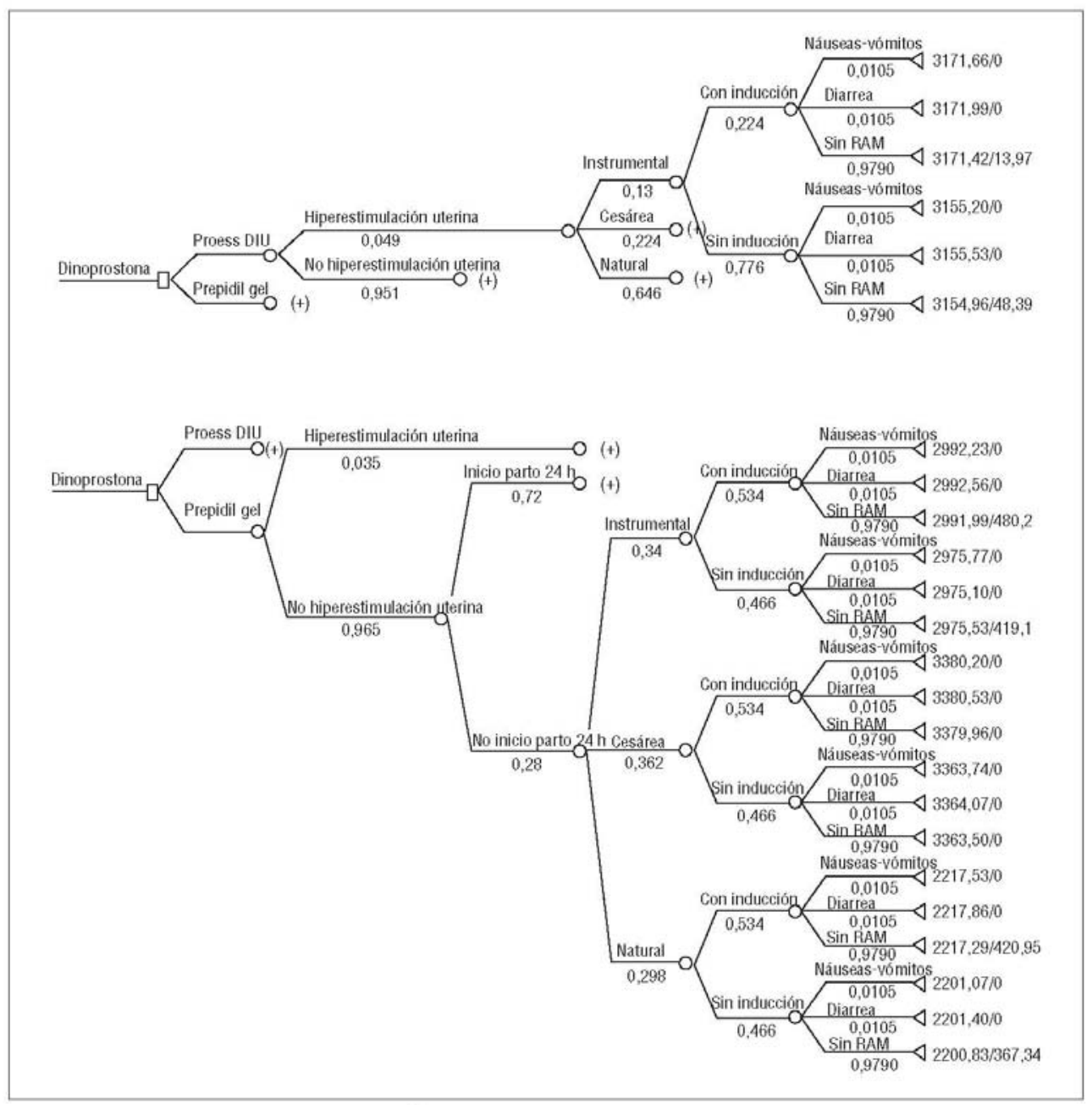

Figura 3 Árbol de decisión del modelo 1 (abreviado). 


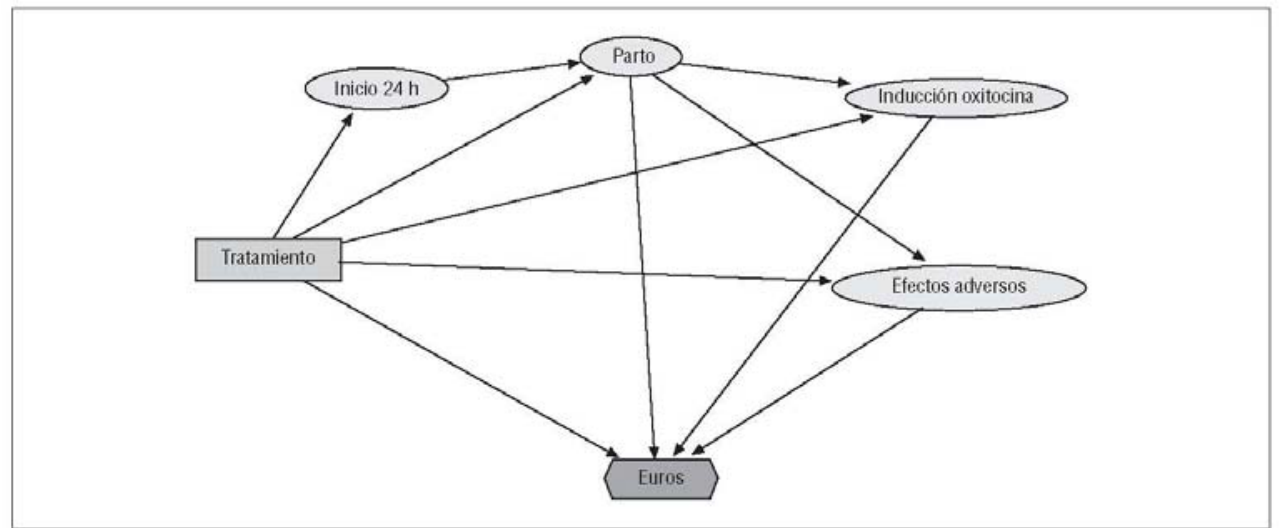

Figura 4 Diagrama de influencia del modelo 2.

Tabla 3 Probabilidades a priori del nodo de efectos adversos en el modelo 2

\begin{tabular}{|c|c|c|c|c|c|c|}
\hline \multirow[t]{2}{*}{ P (eEfectos aAdversos) } & \multicolumn{3}{|c|}{ PROPESS } & \multicolumn{3}{|c|}{ PREPIDIL } \\
\hline & PV instrumental & Cesárea & PV natural & PV instrumental & Cesárea & PV natural \\
\hline Hemorragia post-parto & 0,048 & 0,048 & 0,048 & 0,167 & 0,167 & 0,167 \\
\hline Hiperestimulación uterina & 0,049 & 0,049 & 0,049 & 0,035 & 0,035 & 0,035 \\
\hline Náuseas y vómitos & 0,0105 & 0,0105 & 0,0105 & 0,0105 & 0,0105 & 0,0105 \\
\hline Diarrea & 0,0105 & 0,0105 & 0,0105 & 0,0105 & 0,0105 & 0,0105 \\
\hline Sin RAM & 0,882 & 0,882 & 0,882 & 0,777 & 0,777 & 0,777 \\
\hline
\end{tabular}

Tabla 4 Resultados del modelo 2

\begin{tabular}{lcccccc}
\hline \multicolumn{1}{c}{ Estrategia } & $\begin{array}{c}\text { Coste } \\
\text { en } €\end{array}$ & $\begin{array}{c}\text { Coste } \\
\text { marginal }\end{array}$ & $\begin{array}{c}\text { Efectividad } \\
\left(\mathrm{n} .{ }^{\circ} \text { partos) }\right.\end{array}$ & $\begin{array}{c}\text { Efectividad } \\
\text { marginal }\end{array}$ & C/E & RCEI \\
\hline $\begin{array}{l}\text { Dispositivo intrauterino } \\
\text { Gel endocervical }\end{array}$ & $\begin{array}{l}2.743,5 \\
3.413,1\end{array}$ & 669,6 & $\begin{array}{c}1.857,1 \\
369,9\end{array}$ & $-1.487,2$ & 9,23 & Dominada \\
\hline RCEl: ratio coste-efectividad incremental. & & & & & &
\end{tabular}

\section{Discusión}

Las principales razones que justifican la modelización son: la necesidad de generalización de las intervenciones sanitarias; no siempre los ensayos clínicos son lo duraderos que deberían para recoger todos los eventos de interés; extrapolación de los datos obtenidos de los ensayos clínicos o estudios observacionales, síntesis de información de procesos complejos o el desarrollo de sistemas que generen decisiones basadas en información contrastada ${ }^{3,5}$

Los tipos de modelos pueden clasificarse, dependiendo de su estructura, en árboles de decisión, diagramas de influen. cia, modelos de Markov, modelos de supervivencia, modelos de eventos discretos entre otros. Estos modelos pueden ser determinísticos y estocásticos o probabilísticos, los cuales tienen en cuenta las probabilidades que se dé un resultado por el efecto del azar utilizando técnicas de simulación ${ }^{1-5}$.

Los modelos farmacoeconómicos deben tener las siguientes características: ser explícitos y creíbles clínicamente (justificados), incorporar todas las intervenciones relevantes, ser transparentes y claros (sencillos y entendibles), que los datos incorporados sean válidos, que las asunciones sean debidamente explicadas, y argumentar la metodología utili.

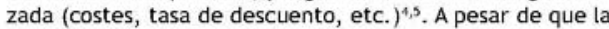
mejora en la metodología utilizada ha apuntalado con más firmeza estos modelos farmacoeconómicos, todavía quedan dudas sobre los mismos para trasladar sus resultados a la 
Resultados y discusión

práctica clínica diaria ${ }^{4 \cdot 7,9}$. El Instituto Nacional Británico para la Salud y la Excelencia Clínica (NICE) ${ }^{10}$ considera los modelos farmacoeconómicos una importante herramienta metodológica para demostrar la eficiencia de las intervenciones evaluadas y por tanto asignar los recursos disponibles, considerando que éstos se utilizarán cada día más, sobre todo si se despejan las dudas de sus detractores, y por tanto es necesaria su estandarización para abandonar la etiqueta de "cajas negras" que les acompañan4.

La incertidumbre es inherente a la toma de decisiones clínicas por lo que la mejor solución es incorporarla al propio modelo planteado ${ }^{2}$. La incertidumbre sobre los costes, los beneficios y las relaciones entre ambos puede tener un doble origen. Por un lado, puede existir incertidumbre acerca de los parámetros. Esto ocurre cuando no puede conocerse con certeza cuáles son los verdaderos valores numéricos de tales parámetros. Por otra parte, podemos enfrentarnos a la incertidumbre sobre el modelo, esto es, incertidumbre acerca de cuál es la forma óptima de combinar los paráme. tros. En efecto, esta incorporación se realiza desde hace tiempo mediante el análisis de sensibilidad, normalmente variando los valores que pueden tomar los parámetros o variables consideradas ${ }^{14}$. Existen dos técnicas para analizar el grado de incertidumbre en farmacoeconomía y son: a) el análisis de sensibilidad cualitativo: univariante y multivariante, análisis de sensibilidad de extremos, análisis de sensibilidad umbral; $y$ b) el análisis estadístico de la incertidumbre que contempla intervalos de confianza del coste efectividad incremental, cajas de confianza, expansión de series de Taylor, elipse de confianza, método Fieller método de remuestreo repetitivo y el análisis de sensibili. dad probabilístico?

No todas las guías incorporan la realización del análisis de sensibilidad estructural, aunque sí describen la metodología para que se minimicen los sesgos en la estructura del modelo seleccionado antes de realizar el estudio. Para minimizar los errores se suelen utilizar checklists que sirven de guía a los propios investigadores o de control a los evaluadores o a los que deciden, a los que va dirigido el informe final $4,6,10-13$. Sobre este tipo de recomendaciones la mayoría establece que debe simplificarse al máximo el modelo, reflejar escrupulosamente la realidad del problema clínico que nos ocupa, establecer claramente el objetivo y la hipótesis de modelo, describir el modelo y su estructura detalladamente, decidir para qué horizonte temporal y perspectiva se utilizará, describir las alternativas que se evalúan, tener en consideración todas las variables importantes que afecten a los resultados tanto económicos como clínicos, establece las fuentes de información tanto de efectividad como de efectos secundarios y costes y, por último, justificar cada una de las elecciones realizadas, así como las asunciones contempladas en nuestro modelo basadas en la mejor evidencia científica disponible ${ }^{4,6,10}$. Por tanto, no es posible incorporar al modelo la situación más improbable o "caso raro" que se nos presente, ya que por una parte aumentaríamos su complejidad y, por otra, su contribución sería prácticamente despreciable para resolver la incertidumbre planteada ${ }^{8}$.

En medicina no se realizan normalmente cambios en la estructura del modelo como ocurre en la ingeniería, la arquitectura, las finanzas o la economía ${ }^{17}$. Cierto es que los resultados obtenidos en intervenciones sucesivas nos puede conllevar resultados muy diferentes e incluso aberrantes, circunstancia que se da en raras ocasiones en otros campos. Por ejemplo, no es posible anteponer la variable muerte a ninguna otra, ya que es un resultado final en sí misma, o poner la administración de un medicamento tras sus efectos secundarios; sin embargo, es posible en la construcción de un puente poner en el árbol de decisión la construcción de la plataforma antes que la de pilotes, o viceversa, de tal manera que se extraiga la situación más eficiente.

En efecto, se ha realizado un trabajo con el objetivo de extraer un documento de consenso o guía a partir de 16 guías o checklists publicados considerados excelentes, para que pueda ser aplicado en la toma de decisiones dentro del propio NICE y cuyo resultado puede ser consultado, determinando la manera y pasos a seguir a la hora de realizar un modelo, garantizando su fiabilidad, validez interna y externa ${ }^{10}$.

Posiblemente, en el campo sanitario no se realizan frecuentemente análisis de sensibilidad estructural, ya que cada uno de los modelos considerados debe ser clínicamente congruente, es decir, debe validarse previamente por clínicos que atienden a los pacientes antes de poder utilizarse.

Otra de las razones es la dificultad metodológica que entrañan, sin minusvalorar los recursos empleados en tiempo y personal para llevarlos a cabo. Sin duda, la utilización de los diagramas de influencia permite omitir en gran parte este último inconveniente, aunque no estén exentos de importantes limitaciones como la mala interpretación en modelos asimétricos, muy común en sanidad, añade restricciones a las variables o utiliza valores de "no observados"; sin embargo, son más intuitivos y su evaluación puede ser automática o por medio de un árbol de decisión, como se ha mostrado en este trabajo ${ }^{17,18}$

La estructura del modelo es claramente una fuente de desconfianza para trasladar sus resultados a la práctica clínica diaria $4,6,10$, siendo el análisis de sensibilidad estructural una herramienta útil para despejar estas dudas, reforzando los resultados obtenidos en las evaluaciones económicas por mejorar su fiabilidad, al igual que sucede en el análisis de sensibilidad de los parámetros y el análisis de sensibilidad probabilístico ${ }^{10.73}$

En este trabajo demostramos que se puede modificar la estructura del modelo respetando su congruencia clínica y sin necesidad de realizar nuevas asunciones que nos podría llevar a una espiral de sinrazones o de estimaciones inco. rrectas de los propios parámetros.

Al comparar cuantitativamente el modelo 2 con el modelo base, al considerar exactamente los mismos valores o resultados a priori pero en variables diferentes, se obtienen resultados del análisis coste-efectividad similares. Al eliminar una variable cuya incidencia es muy baja, como las hemorragias posparto debidas al tratamiento, se comprueba que aunque no varía la decisión final en la toma de decisiones, la variación de los resultados obtenidos en el modelo 1 es mayor respecto al modelo base. Con ello deducimos que se debe tener en cuenta la variable hemorragias posparto en el modelo, independientemente de si se incorpora de manera independiente o en la variable de efectos adversos ${ }^{14}$. Posiblemente, se corrobore con este ejemplo que el análisis de sensibilidad estructural realizado en modelos sencillos no afecte a los resultados ${ }^{6}$, quedando sin clarificar si se debe aplicar de manera sistemática. 
Resultados y discusión

La modificación de la estructura del modelo nos conduce a determinar qué modelo es más sencillo, transparente fiable, y por tanto, más entendible por todos los implicado en el proceso de toma de decisiones, lo que conduce a seleccionar el mejor modelo posible en una situación clínica determinada y explicar el porqué de la misma. Esta selección puede incluso ahorrarnos recursos, eliminando varia bles o costes del modelo al no influir en los resultados finale estudiados o en la eficiencia comparada de las intervenciones, por ejemplo, el análisis coste-utilidad o coste efectividad incremental.

Las conclusiones que podemos extraer o inferir de la realización de un análisis de sensibilidad estructural en los estudios farmacoeconómicos son:

- Es posible realizar en el campo sanitario un análisis de sensibilidad estructural congruente con la situación clínica estudiada.

- Cada uno de los modelos propuestos para realizar el análisis de sensibilidad estructural debe ser validado clínicamente.

- La utilización de diagramas de influencia permite llevar a cabo el análisis de sensibilidad de manera más intuitiva y sin grandes esfuerzos, respecto a los árboles de decisión.

- Aunque la reducción de la incertidumbre obtenida con el análisis de sensibilidad estructural puede no ser muy alta, sin duda aporta información valiosa sobre el problema que se quiere representar $y$ da una mayor validez y fiabilidad al modelo.

\section{Conflicto de intereses}

Los autores declaran no tener ningún conflicto de intereses, actual o pasado, con lo que se expone en el presente artículo ni con los laboratorios que comercializan los medicamentos mencionados en el mismo.

\section{Bibliografía}

1. Barr JT, Schumacher GE. Decision analysis and pharmacoeco nomics evaluations. En: Bootman JL, McGhan WF, Townsend RJ, eds. Principles of Phamacoeconomics. Houston: Harvey Whit ney Books; 2004:175-210.
2. Drummond MF, Stoddart GL, Torrance GW, Sculpher MJ, O'Brien $\mathrm{BJ}$, eds. Methods for the Economic Evaluation of Health Care Programmes. Oxford: Oxford Univ Pr; 2005.

3. Rubio Cebrián $\mathrm{S}$. Análisis de decisión y tratamiento de la incertidumbre. En: Gimeno JA, Rubio S, Tamayo P, eds. Economía de la salud: instrumentos. Manuales de Dirección Médica y Gestión Clínica. Madrid: Ediciones Díaz de Santos; 2006: 157-9.

4. Soto J. Health economic evaluations using decision analytic modeling. Principles and practices-utilization of a checklist to their development and appraisal. Int J Technol Assess Health their development and

5. Rodríguez Barrios JM. Papel de los modelos en las evaluaciones económicas en el campo sanitario. Farm Hosp. 2004;28:231-42.

6. ISPOR Task Force. Principles of good practice for decision analytic modeling in health-care evaluation. Value Health. 2003;6:9-17.

7. Rubio-Terrés C, Cobo E, Sacristán JA, Prieto L, Del Llano J, Badia X. Análisis de la incertidumbre en las evaluaciones económicas de intervenciones sanitarias. Med Clin. 2004 ,

. Brigs AH. Handling uncertainty in economic evaluation. En: Briggs AH. Handting uncertainty in economic evaluation. En: Drummond M, McGuire A, eds. Economic Evaluation. Merging theory with practice. London: Office of Health Economics, Oxford University Press; 2001.

9. Willan AR, Briggs AH, eds. Statistical analysis of cost. effectivenes data. The Atrium, West Sussex: John Wiley \& Sons Ltd; 2006.

10. Philips Z, Ginnelly L, Sculpher M, Claxton K, Golder S, Riemsma $R$, et al. Review of guidelines for good practice in decision-ana ytic modelling inhealth technology assessment. Health Techno

11. Akehurst R, Anderson P, Brazier J, Brennan A, Briggs A, Buxton $M$, et al. Decision analytic modelling in the economic evaluation of health technologies-a consensus statement. Pharmacoeconomics. 2000;17:443-4.

12. Hay JW. Economic modeling and sensitivity analysis. Value Health. 1998;1:187.93

13. Ramsey SD, Sullivan SD. Weighing the economic evidence: guidelines for critical assessment of cost-effectiveness analy. guidelines for critical assessment of cost-ctfoct.

14. Carrera-Hueso FJ, Poquet Jornet JE, Ramón Barrios A, Conde Femández F. Coste-efectividad en la inducción del parto con dinoprostona. Aten Farm. 2010;12:359-68.

15. DATA* v 3.5. TreeAge Software Inc. [citado 20 de ene 2011]. Disponible en: http://www.treeage.com/index.htm

16. Elvira" versión 0.162. UNED [citado 16 de feb de 2010]. Disponible en: http://www.ia.uned.es/-elvira/instalar/instalar.html

17. Ríos Insua S, Bielza Lozoya C, Mateos Caballero A, eds. Funda. mentos de los sistemas de ayuda a la decisión. Madrid: RA.MA Editorial; 2002.

18. Díez Vegas FJ. Probabilidad y teoría de la decisión en medicina. Herramientas de Gestión e investigación sanitaria. Madrid: UNED; 2005. 


\section{Capítulo 8}

Amat Díaz M, Poveda Andrés JL, Carrera-Hueso FJ. Aplicación de la farmacoeconomía en la gestión clínica. Farm Hosp 2011; 35 (Supl 1): 18-24. 


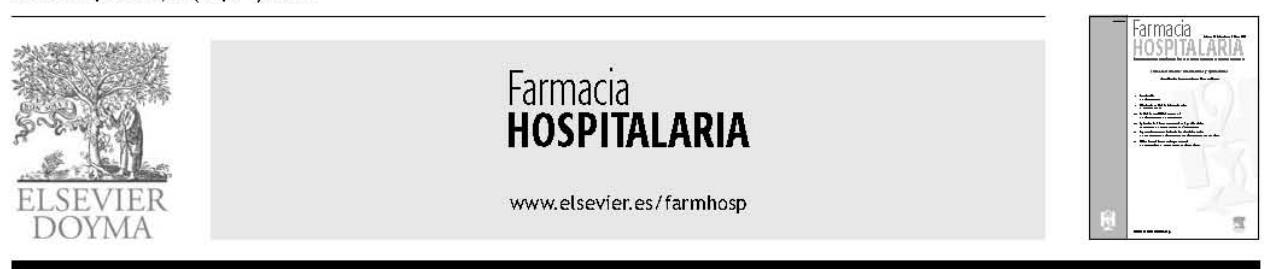

\section{Aplicación de la farmacoeconomía en la gestión clínica}

\section{Amat Díaz ${ }^{\mathrm{a}, *}$, J.L. Poveda Andrés ${ }^{\mathrm{b}}$ y F.J. Carrera-Huesoc}

aServicio de Farmacia, Hospital Universitario y Politécnico La Fe, Valencia, España

- Jefe del Servicio de Farmacia, Hospital Universitario y Politécnico La Fe, Valencia, España

'Servicio de Farmacia, Hospital Dr. Moliner, Serra, Valencia, España

PALABras Clave Farmacoeconomía; Gestión clínica;

Eficiencia;

Toma de decisiones;

Excelencia

\section{KEYWORDS}

Pharmacoeconomics;

Clinical management:

Efficiency;

Decision making:

Excellence
Resumen Se expone la importancia de la gestión clínica en la transformación de las organizaciones y su implicación en el quehacer diario del profesional sanitario y, específicamente, del farmacéutico de hospital. Los cambios sociales obligan a la adaptación de los modelos de asistencia sanitaria hacia nuevas formas de gestión, desde modelos gerenciales más rígidos hacia modelos organizativos basados en la gestión clínica. Desde esta perspectiva, se aborda la farmacoeconomía como una herramienta útil para introducir el criterio de eficiencia en todas las decisiones sujetas a la gestión clínica, incluidas las farmacoterapéuticas. Posteriormente, se muestra la aplicación de esta disciplina en situaciones reales de decisión y se proponen ámbitos para su utilización dentro del marco de trabajo de la farmacia hospitalaria.

(0) 2011 SEFH. Publicado por Elsevier España, S.L. Todos los derechos reservados.

\section{Application of pharmacoeconomics in clinical management}

Abstract The present article discusses the importance of clinical management in the transformation of organizations and its role in the daily activities of health professionals and, in particular, of hospital pharmacists. Because of social changes, healthcare models must make the shift from more rigid management models toward new organizational models based on clinical management. From this perspective, pharmacoeconomics is viewed as a useful tool to introduce the criteria of efficiency in all decisions subject to clinical management, including those on pharmacotherapeutics. Subsequently, the application of this discipline is discussed in real decision-making scenarios and settings for its use within the context of the work of hospital pharmacy are proposed.

(1) 2011 SEFH. Published by Elsevier España, S.L. All rights reserved.

Autor para correspondencia.

Correo electrónico: amat mardia@gva.es (M. Amat Díaz).

1130-6343/\$ - see front matter (c) 2011 SEFH. Publicado por Elsevier España, S.L. Todos los derechos reservados. 
Resultados y discusión

\section{Gestión clínica}

Desde la creación de la Comisión de Reformas Sociales, en 1883 , como punto de partida de una política de protección de la clase obrera', mucho se ha evolucionado hasta dar forma a lo que conocemos como Sistema Nacional de Salud (SNS), cuyo marco normativo básico es la Ley 14/1986 General de Sanidad. EI SNS, regido bajo los principios de unidad, universalidad, equidad y solidaridad, ha resultado un instrumento esencial para garantizar el Estado de Bienestar, contribuyendo, así, a la cohesión económica y social de nuestro país.

El resultado de esta transformación ha sobrevenido, por tanto, en un modelo de asistencia sanitaria "de todos y para todos", consagrando uno de los derechos fundamentales de los seres humanos, el derecho a la protección de su salud, tal y como recoge nuestra Carta Magna. Asimis mo, en su evolución, el SNS ha sabido plasmar su capacidad para proporcionar resultados óptimos en relación a distintos parámetros referentes al estado de salud de la población. Otros resultados de salud alcanzados se han atribuido a la acción del propio sistema sanitario, entre ellos, los relacionados con parámetros de cobertura, acceso y equidad financiera, resultados relacionados con la calidad y seguridad de la atención sanitaria, así como, los referentes al grado de satisfacción de los usuarios y su le gitimación por parte de la población ${ }^{2}$. Y todo ello, aparen temente, a un "buen precio", traducido con un porcentaje del producto interior bruto (PIB) dedicado a sufragar el gasto sanitario por debajo del promedio europeo y situado en un $9 \%$ para 2008 , según los últimos datos proporciona. dos por la Organización para la Cooperación y el Desarrollo Económicos $(\mathrm{OCDE})^{3}$.

Pero sería equívoco caer en la euforia momentánea que puede desprenderse de los logros expuestos. Así, diferentes factores como el envejecimiento de la población, la mayor complejidad de los problemas de salud, el incremento sustancial de sus necesidades y exigencias socio-sanitarias, la inmigración, el aluvión constante de nuevas tecnologías e incluso la amplia variabilidad en la práctica médica, entre muchos otros, pueden dificultar la sostenibilidad del modelo sanitario, más si éste se soporta en base a las recaudaciones fiscales y, por ende, está confinado a los límites de las contribuciones, modulables, a su vez, según el marco económico general del Estado.

Por ello, surge la necesidad de adaptar nuestros modelos de salud, no sólo en cambios que se traduzcan en mejoras de la práctica médica, sino también en la creación de nuevas fórmulas de gestión para las organizaciones sanitarias. De esta forma, resulta preciso desarrollar y aplicar iniciativas que permitan afrontar el reto que se nos plantea. Estas medidas pueden abordarse a nivel macro, es decir, a través de diferentes políticas de salud, si bien, también pueden ser aplicadas desde una dimensión meso, corres pondiente a la perspectiva de las propias organizaciones sanitarias, pero lo que realmente resulta crucial es avan zar hacia organizaciones horizontales, dando valor a los microsistemas y con ello a los profesionales sanitarios que lo conformant. Centrar la toma de decisiones en el propio clínico, en aras del reconocimiento de sus valores profesionales, no es más que una apuesta hacia la consecución de organizaciones más eficientes, lo que implica no sólo la prestación de una asistencia de calidad, sino hacerlo, además, sin malgastar los recursos 5 .

La idea de gestión clínica puede formularse en un doble sentido: hacer más clínica la gestión y hacer más gestora la clínica. A través de ambos componentes se facilita la identificación de aspectos importantes de este patron ${ }^{6}$. En efecto, para hacer más clínica la gestión, el reto se centra en tres ámbitos ${ }^{6,7}$ :

- Gestionar lo esencial, es decir, la función de producción, reforzando el papel y dinamismo de las direcciones asis. tenciales y los objetivos e información de efectividad y calidad.

- La gestión del conocimiento, para aportar desde la red sanitaria una política científica y unos instrumentos de apoyo a las mejores prácticas y a la excelencia.

- La gestión de las interdependencias de red, para abordar los problemas de cronicidad y de respuestas integrales a patologías desde una infraestructura y una logística de red.

Introducir la dimensión gestora en el mundo clínico supone avanzar en varios sentidos ${ }^{6}$

- Buscar formas de organizar los centros, servicios y unidades para que domine la cooperación, la horizontalidad y el trabajo por procesos.

- Fomentar un liderazgo desde el mundo clínico que profesionalice los retos y defienda los proyectos de cambio organizativo en su entorno profesional.

- Mejorar y racionalizar las decisiones clínicas, buscando mejorar las dimensiones de efectividad, eficiencia, calidad y satisfacción de los pacientes.

Así, aceptar las bases de la gestión clínica conlleva para los profesionales sanitarios, la asunción de responsabilida. des más allá de lo estricto de su tarea y, con ello, la obligación de disponer de una aptitud clínica superior que aúne sus habilidades cotidianas junto a conocimientos específicos, en albor de un mayor compromiso institucional y social $^{7}$. La medicina y la gestión, de esta forma, deben caminar juntas con el único fin de conseguir los mejores resultados para los pacientes y al mismo tiempo conseguirlos de la mejor forma posible. Es el camino, por tanto, para garantizar la excelencia, meta de cualquier profesional sanitario.

La gestión clínica, como paradigma emergente de esta década, resulta la piedra angular de las estrategias de mejora del sistema y de búsqueda de sostenibilidad y excelencia. Su avance depende de la factibilidad de una nueva alianza entre políticos, gestores, médicos y resto de profesionales sanitarios, alineando intereses, compartiendo visiones y minimizando los conflictos de interés ${ }^{6}$.

Hemos de pasar a ser, por tanto, los protagonistas del cambio, los elementos clave para asegurar que nuestro sistema sanitario persevere. No podemos olvidar que cada una de las decisiones que tomamos en nuestra práctica diaria va vinculada a una parte del gasto sanitario. Es necesario ser un aval para la racionalización de los recursos, aunque siempre sin eludir el beneficio de aquellos a los que se dirige nuestra atención y nuestra razón de ser, los pacientes. 
Resultados y discusión

\section{Otros factores a considerar}

La incorporación de la atención farmacéutica como paradig ma del rol del farmacéutico y el compromiso explícito que supone la corresponsabilidad en los resultados clínicos, humanísticos y económicos, constituye el pílar sobre el que sustentar el nuevo modelo de actividad en la farmacia de hospital.

La atención farmacéutica no es una tecnología, desde el punto de vista economicista, fácil de medir. En efecto, exis ten multitud de estudios que demuestran su utilidad y efi. ciencia para el sistema sanitario, pero muchos están salpicados de sesgos de diseño, análisis incorrectos, muestras pequeñas y períodos de seguimiento excesivamente cortos?.

Es por ello que el farmacéutico de hospital debe ser capaz de obtener $\mathrm{e}$ interpretar las variables o resultados en salud, tanto de tipo clínico, epidemiológico como económico, pero también debe considerar los resultados humanísticos, que son los de calidad de vida relacionada con la salud y de sa. tisfacción percibida por los pacientes.

Es necesario que desde el punto de vista de nuestra organización seamos capaces de crear, adquirir y transmitir conocimiento. La gestión del conocimiento pasa a ser, por tanto, una herramienta clave para que las organizaciones sean excelentes, y esencial en las sanitarias.

Los servicios de farmacia hospitalarios (SF) son elementos vertebradores del hospital y tienen una visión global del centro hospitalario. Además, son generadores y transmisores de conocimiento. Cada SF debe conocer y desarrollar S capital intelectual y orientarlo siempre hacia el paciente ${ }^{9}$ Con ello, añadirá valor y calidad a la mayoría de procesos asistenciales, tanto los desarrollados dentro de los mismos como los compartidos.

La organización horizontal significa que se deben alinear las necesidades de los profesionales y los pacientes con las estrategias de la institución, y no, solamente, implantar una gestión por procesos. Esto implica impulsar estrategias de innovación y de flexibilidad operativa y financiera en su organización. Para innovar, es necesario pensar en los resulta dos que se obtienen tanto en los propios SF como los obtenidos en otros servicios y departamentos del hospital, consiguiendo una mejor gestión asistencial. Con ello, fomentamos la creación de la cadena de la innovación en la que participen tanto empleados como clientes, internos y externos, en el SF.

\section{La farmacoeconomía como herramienta}

Sin duda, ante la envergadura del reto que se nos plantea debemos estar preparados y ser conocedores de los instrumentos disponibles. La toma de decisiones debe estar nece sariamente fundamentada sobre unos criterios de priorización que nos permitan asignar recursos eficientemente. Consecuentemente, el profesional sanitario debe disponer de las herramientas que le permitan fijar dichos criterios a la hora de elegir entre distintas alternativas.

Atisbar el entorno es el preludio para generar soluciones. Como ya hemos anticipado, uno de los cuantiosos determinantes que están intimidando a nuestro sistema sanitario resulta de la importante repercusión que ha tenido la incor- poración de nuevas tecnologías en el ámbito de la salud. Tanto es así, que se considera uno de los principales factores causantes del crecimiento del gasto sanitario y de la prestación sanitaria media ${ }^{10}$. El incremento del gasto que supone la incorporación de los avances tecnológicos no ha contribuido en la misma medida en la producción de valor social a las poblaciones que lo soportan ni tampoco se han obtenido resultados beneficiosos tan evidentes para los pacientes".

En este punto, cabe hacer una mención especial a la influencia que en esta eclosión tecnológica ha supuesto la in. troducción de nuevos medicamentos en las organizaciones sanitarias. Así, y según datos facilitados por el Strategic Re. search Center Bussines School ${ }^{12}$ en el informe emitido re. cientemente sobre el gasto farmacéutico en España, referenciado al año 2007 , situaba en un $21 \%$ el gasto farmacéutico sobre el gasto sanitario total en nuestro pais, al mismo nivel que países como Italia y República Checa.

Estas cifras, que pueden llegar a ser preocupantes, aún más si cabe, hacen necesaria la colaboración de todos los que formamos parte del sistema sanitario. Desde las administraciones públicas son muchas las medidas que se están valorando para dotar la situación de un mayor control. EI Consejo Interterritorial del SNS aprobó un documento marco con directrices para promover la calidad, la equidad, la cohesión y la sostenibilidad del SNS el pasado 18 de marzo del $2010^{13}$. Entre las acciones propuestas, y en materia de eficiencia del sistema y control del gasto, se señala la importancia de la incorporación de medicamentos en la cartera de servicios del sistema sanitario bajo criterios de coste-efectividad. De la misma manera, se apunta a que la construcción de guías farmacoterapéuticas, como apoyo a la toma de decisiones, se encuentre fundamentada sobre criterios de evidencia y coste-efectividad.

La inclusión de consideraciones de eficiencia en la toma de decisiones parece refundir en un axioma que, a pesar de no caracterizarse por su singularidad, está cada día más patente. Desde principios de los años noventa, en Australia y Canadá, y especialmente con la creación en el Reino Unido del Instituto Nacional Británico para la Salud y Excelencia Clínica en el año 1999, conocido como NICE, que incluye la evaluación en la selección de innovaciones sanitarias la adopción de la evaluación económica en la toma de decisiones se ha convertido en una necesidad inminente, a la que debe darse la oportuna respuesta de carácter práctico y aplicable ${ }^{14}$.

En este sentido, la farmacoeconomía parece emerger de nuevo, constituyéndose como una disciplina cardinal en nuestro quehacer y que permite aproximar al profesional sanitario a un escenario más acorde a nuestras posibilida. des. La farmacoeconomía nos proporciona información sobre todos los aspectos económicos en el sector sanitario, aportándonos una perspectiva más amplia en el abordaje del análisis de todas las implicaciones del medicamento a través de herramientas metodológicas específicas ${ }^{15}$. Dentro de los análisis farmacoeconómicos (tabla 1) se incluyen las evaluaciones económicas, técnicas que nos permiten calcular la eficiencia en función de la relación entre los costes y consecuencias de intervenciones alternativas ${ }^{16}$.

La realidad de la aplicación de la farmacoeconomía en la práctica diaria puede verse reflejada en estudios recientes como el llevado a cabo por Catalá-López $\mathrm{F}$ et $\mathrm{al}^{17}$. En él se 
Resultados y discusión

Tabla 1 Técnicas comúnmente utilizadas para el análísis económico en el sector sanitario ${ }^{15}$

\begin{tabular}{|c|c|c|c|}
\hline Técnica/tipo de análisis & Características diferenciales & Evaluación económica & Fármaco-economía \\
\hline Análisis de costes & $\begin{array}{l}\text { Análisis de los costes asociados a una o más } \\
\text { intervenciones }\end{array}$ & & $x$ \\
\hline $\begin{array}{l}\text { Análisis de minimización } \\
\text { de costes }\end{array}$ & $\begin{array}{l}\text { Análisis de los costes de dos o más } \\
\text { intervenciones cuya efectividad } \\
\text { se haya mostrado equivalente }\end{array}$ & $x$ & $x$ \\
\hline Análisis de coste-efectividad & $\begin{array}{l}\text { Análisis de los costes y beneficios de dos } \\
\text { o más intervenciones. Los beneficios se } \\
\text { miden en términos de unidades naturales }\end{array}$ & $x$ & $x$ \\
\hline Análisis coste-utilidad & $\begin{array}{l}\text { Análisis de los costes y beneficios de dos } \\
\text { o más intervenciones. Los beneficios } \\
\text { se miden en AVAC }\end{array}$ & $x$ & $x$ \\
\hline Análisis coste-beneficio & $\begin{array}{l}\text { Análisis de los costes y beneficios de dos } \\
\text { o más intervenciones. Los beneficios se } \\
\text { miden en términos de unidades monetarias }\end{array}$ & $x$ & $x$ \\
\hline $\begin{array}{l}\text { Análisis de costes } \\
\text { y consecuencias }\end{array}$ & $\begin{array}{l}\text { Análisis de los costes y beneficios de dos } \\
\text { o más intervenciones. Los beneficios se } \\
\text { miden en distintas unidades, sin integrarse } \\
\text { en un indicador único con los costes }\end{array}$ & $x$ & $x$ \\
\hline $\begin{array}{l}\text { Estudio del coste } \\
\text { de la enfermedad }\end{array}$ & $\begin{array}{l}\text { Análisis de los costes asociados } \\
\text { a una enfermedad o factor de riesgo }\end{array}$ & & $x$ \\
\hline $\begin{array}{l}\text { Análisis del impacto } \\
\text { presupuestario }\end{array}$ & $\begin{array}{l}\text { Análisis del impacto agregado, en términos } \\
\text { financieros, de la introducción de una nueva } \\
\text { intervención en una población determinada }\end{array}$ & & $x$ \\
\hline
\end{tabular}

describen las principales características de los trabajos de evaluación económica de intervenciones sanitarias realizados en España durante el período comprendido entre 1983 y 2008. Se muestra una evolución creciente del número de evaluaciones económicas de intervenciones sanitarias, des. tacándose, además, un crecimiento máximo en los últimos cinco años. El ámbito de la mayoría de estudios analizados se situaba en atención especializada.

Con estos datos, parece indudable afirmar que la utilización de las evaluaciones económicas resulta cada día más imprescindible en nuestro ámbito de actividad. Aún así, y a pesar de encontrarnos en el camino correcto, resulta ineludible evocar esfuerzos para que la aplicación de estas herramientas en la toma de decisiones sea una constante en la actividad clínica.

Es prioritario identificar las posibles barreras para la utilización de las evaluaciones económicas por parte de los clínicos, las cuales se detallan en la tabla $2^{18}$. Las principales razones, documentadas por los propios profesionales sanitarios, que dificultan la implantación de criterios de eficiencia en la toma de decisiones son, por un lado, la dificultad que radica en la comprensión de este tipo de estudios o bien en el abordaje que suelen realizar y que se aleja, más bien, de la práctica diaria. Otras argumentaciones que cuestionan la credibilidad de las evaluaciones económicas, son que estos estudios, en la mayoría de ocasiones, se encuentran financiados por las industrias farmacéuticas y, también, surgen de la idea equívoca de entender las evaluaciones económicas como herramientas puramente de ahorro y no de mejora de calidad.

Circunscritos los problemas, sólo cabe la puesta en marcha de soluciones. Así, y tras definir un marco en el que la
Tabla 2 Barreras e incentivos para el uso de evaluaciones económicas de intervenciones sanitarias por los profesionales de la salud18

Barreras

Problemas éticos

Existencia de otros criterios considerados más relevantes Falta de tiempo

Poco conocimiento sobre los métodos de evaluación

El objetivo real es ahorrar y no lograr mayor eficiencia

Problemas de fiabilidad de los estudios

Los estudios no abordan los problemas diarios

aplicación de la farmacoeconomía parece ser un valor en alza en nuestro medio, pero que aún así hay que incentivar, conviene establecer instrumentos que permitan afrontar los problemas que aún parecen advertirse para su utilización en la toma de decisiones clínicas. A razón de esto, se ha publicado recientemente una propuesta de guía para la evalua. ción económica de intervenciones sanitarias ${ }^{19}$ (tabla 3) con la finalidad de ofrecer una serie de reglas que orienten al clínico sobre cuestiones técnicas y metodológicas tanto para la realización de evaluaciones económicas como para su análisis. Estas recomendaciones se encuentran construidas sobre 17 ítems bajo los que se establecen los requisitos que deben cumplir este tipo de estudios, resultando en una mayor garantía desde el punto de vista de la calidad, puesto que es importante no olvidar que el fundamento de las evaluaciones económicas es facilitar la toma de decisiones. 
Resultados y discusión

Tabla 3 Lista de comprobación para el análisis y realización de las evaluaciones económicas ${ }^{19}$

Sí No En parte No procede

1. Pregunta de investigación y objetivos del estudio

¿El estudio establece claramente su objetivo y la pregunta de investigación?

¿La evaluación económica se efectúa de modo general y posteriormente, si los datos

$\square \quad \square \quad \square \quad \square$

lo permiten, en subgrupos de población (edad, sexo, gravedad, niveles de riesgo)

$\square \square \square \square$

en el caso de que haya diferencias relevantes en la efectividad o en los costes entre ellos?

2. Perspectiva

¿La evaluación económica incluye tanto la perspectiva social como la del financiador (Sistema Nacional de Salud)?

¿Se presentan ambas perspectivas de forma separada y diferenciada?

3. Opciones a comparar

¿Se compara la tecnología con, como mínimo, la práctica clínica habitual?

¿Se justifica claramente la elección de la opción a comparar?

4. Tipos de evaluación

¿Se justifica de forma razonable el tipo de análisis elegido en relación a la cuestión planteada?

5. Datos de eficacia/efectividad

¿Se detalla con precisión la fuente utilizada para la obtención de datos de eficacia

y efectividad?

¿Se detallan con precisión el diseño y los métodos utilizados?

6. Medidas de resultados

¿Las medidas de resultados seleccionadas son clínicamente relevantes

(medida de eficacia/efectividad final)?

¿Las escalas sociales de valoración de la calidad de vida relacionada con la salud

han sido validadas a partir de una muestra representativa de la población?

7. Utilización de recursos y costes

¿Se ajustan los costes recogidos a la perspectiva del análisis seleccionada?

¿Se han separado y se detallan de forma adecuada las unidades físicas de los costes y los datos de costes?

8. Horizonte temporal de los costes y beneficios

¿Está el horizonte temporal considerado el más adecuado para captar todos los efectos

diferenciales de las tecnologías evaluadas sobre la salud y los recursos empleados?

9. Modelización

En el caso de aplicar técnicas de modelaje, ¿se justifica y es transparente la elección

del modelo utilizado, asi como los parámetros y supuestos clave de éste?

10. Descuentos para costes y beneficios

¿Se descuentan los costes y resultados que se producen en el futuro empleando la misma tasa?

11. Variabilidad e incertidumbre

¿Se realizó un análisis de sensibilidad?

¿Se justificaron los parámetros clave del estudio y la distribución estadistica de las variables analizadas en el análisis de sensibilidad?

12. Equidad

En caso de incorporar argumentos de justicia social en la evaluación (análisis de equidad), ise presenta este análisis de forma disgregada al caso principal y son transparentes los argumentos empleados?

13. Transferibilidad de resultados

¿Permite el informe extraer conclusiones sobre la transferibilidad o extrapolación

de los resultados a otros contextos?

14. Presentación de resultados

¿Se presentan los resultados mediante un análisis incremental (ICER) y a su vez de forma desagregada (costes y resultados de las alternativas)?

15. Limitaciones del trabajo

¿Se presentan de manera crítica y transparente las limitaciones o puntos débiles del análisis?

16. Conclusiones

¿Las conclusiones del estudio responden a la pregunta de investigación y se desprende

claramente de los resultados obtenidos?

17. Conflicto de intereses

¿Se indica quién encarga, apoya o financia el estudio?

¿Se informa sobre la posible existencia de conflictos de intereses?

$\square \square \square \square$

$\square \square \square \quad \square$

$\square \square \square \square \square$

$\square \square \square \quad \square$

$\square \square \square \square$

$\square \square \square \square$

ㅁ

$\square \quad \square \quad \square \quad \square$

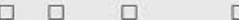

$\square \quad \square \quad \square \quad \square$

$\square \square \square \square$

ㅁ $\square \quad \square$

$\square \square \square \square$

$\square \square \square \square$

$\square \square \square$

ㅁ $\square \square \square$

$\square \square \square \square$

$\square \square \square \square$

$\square \square \square \square$

$\square \square \square \square$

ㅁ $\square$ ㅁ

믐


Resultados y discusión

\section{Una visión más práctica}

Es un hecho evidente que existe la necesidad de introducir elementos de gestión en los hospitales, y en este marco, los estudios farmacoeconómicos pueden servirnos de ayuda en diferentes aspectos ${ }^{20}$.

- Ayuda a estructurar y a articular de forma sistemática y explícita la información a considerar cuando se toma una decisión clínica.

- Ayuda a la distribución adecuada de recursos.

- Apoyo a la elaboración de formularios, protocolos y guías de práctica clínica.

- Apoyo a las decisiones de investigación.

- Apoyo a la justificación de la actividad profesional.

- Ayuda a conseguir el equilibrio entre profesionales.

Todas estas aplicaciones pueden inferir directamente en cada una de las fases del proceso farmacoterapéutico en torno al medicamento: selección, prescripción, preparación, dispensación, administración y seguimiento. De esta forma, los estudios farmaeconómicos pueden servir de base al clínico para fundamentar sus decisiones, incorporando tanto resultados en salud como económicos, a la hora de elegir el tratamiento más adecuado para un paciente, y que, en muchas ocasiones, se caracteriza por un halo de incertidumbre. Permiten incluir medicamentos en formularios y guías farmacoterapéuticas en función de criterios de eficiencia, lo que resulta primordial para la racionalización de la farmacoterapia y conforman, además, una orientación para los agentes decisores a la hora de redistribuir los recursos, consiguiendo, así, una mayor rentabilidad del presupuesto ${ }^{21}$.

En este contexto, los farmacéuticos de hospital, y como consecuencia de su cometido en las organizaciones sanitarias, poseen una situación estratégica tanto para promover como para poner en práctica el uso de los análisis farmacoeconómicos. Entre los estudios realizados en este campo nuestro equipo ha aplicado el análisis coste-beneficio en la implantación de los sistemas automáticos de dispensación de medicamentos en el área de pacientes críticos ${ }^{22}$, los análisis de minimización de costes para la valoración de alternativas terapéuticas, por ejemplo, entre darbepoetina alfa y epoetina alfa en pacientes con insuficiencia renal crónic sometidos a hemodiálisis ${ }^{23}$, o los análisis de coste-efectividad en el tratamiento antifúngico de pacientes hematológi$\cos ^{24}$. Estos trabajos son reflejo de la utilidad real que posee el empleo de las evaluaciones económicas en nuestra actividad asistencial y deben ser un apoyo imprescindible para la toma de decisiones en:

- Asignar los recursos disponibles a las tareas más eficien tes dentro de todo el personal de los SF.

- Reorganizar sus funciones y actividades orientándolas hacia los pacientes, a la vez que contribuye a mejorar ta estructura horizontal de la organización.

- Analizar y evaluar las nuevas tecnologías, haciendo un seguimiento de su grado de penetración en la institución.

- Realizar informes sobre medicamentos y tratamientos farmacoterapéuticos con mayor independencia para las distintas comisiones clínicas, especialmente para la de farmacia y terapéutica, al tener en cuenta la eficiencia de los mismos.
- Identificar oportunidades de mejora que sean más eficientes y, por tanto, su puesta en marcha será más fácilmente al ser apoyadas por la dirección.

- Descentralizar su actividad llevándola al pie de la cama, al poder demostrar el valor añadido que proporcionan los programas de atención farmacéutica que se lleven a cabo.

- Mejorar o reforzar su incorporación al equipo multidisci. plinar al liderar la utilización de los estudios de resultados en salud, incluyendo los de calidad de vida y de satisfacción de los pacientes.

- Utilizar la mejor evidencia científica disponible, por lo que toma parte y se implica en la gestión clínica de manera directa al distribuir y asignar recursos que se utilizan en el proceso farmacoterapéutico. El $70 \%$ de la asignación de recursos de un hospital se realiza en el ámbito clínico.

- Mejorar la función de soporte del proceso farmacoterapéutico que realiza y responsabilizarse de los resultados obtenidos con una visión más independiente de la distribución de recursos más equitativa.

- Realizar evaluaciones farmacoeconómicas en su ámbito de trabajo, bien liderando estudios naturalísticos con diseños pragmáticos, bien mediante la modelización. También puede extrapolar los estudios de la literatura adaptándolos con datos propios de su hospital ${ }^{25}$.

\section{Conclusión}

Las transformaciones sociales obligan a la adaptación de los modelos de asistencia sanitaria hacia nuevas formas de gestión, desde modelos gerenciales más rígidos hacia modelos organizativos basados en la gestión clínica.

El servicio de farmacia, como eje vertebrador de la far. macoterapia que se aplica en el hospital, debe ejercer un liderazgo que necesariamente obliga a incorporar la farma. coeconomía como herramienta para introducir el criterio de eficiencia en todas sus decisiones sujetas a la gestión clinica.

\section{Conflicto de intereses}

Los autores declaran no tener ningún conflicto de intereses, actual o pasado, con lo que se expone en el presente ar. tículo ni con ninguna empresa pública o privada, asegurando su independencia.

\section{Bibliografia}

1. Ministerio de Sanidad, Política Social e Igualdad. Organización Institucional: nuestros orígenes [citado 20 dic 2010]. Disponible en: http://www.ingesa.msc.es/organizacion/origenes/home. $\mathrm{html}$

2. García Armesto S, Abadía B, Durán A, Bernal E. Health Systems in transition: Espana. Análisis del sistema sanitario 2010 [monografia en internet]. Sociedad Espaniola de Salud 2010 [mán pública y Administración Sanitaria; 2010 [citado 10 ene 2011]. Disponible en: http://www.sespas.es/adminweb/uploads/ docs/HiT2010.pdf 
Resultados y discusión

3. Organisation for Economic Co-operation and Developement (OECD). Health Data 2010: key indicators; 2010 [citado 12 ene 2011]. Disponible en: http://www.irdes.fr/EcoSante/ DownLoad/OECDHealthData_FrequentlyRequestedData.xls

4. Bernal Delgado E, Ortún-Rubio V. La calidad del Sistema Nacional de Salud: base de su deseabilidad y sostenibilidad. Gac Sanit. 2010;24:254-8.

5. Martin LA, Neumann CW, Mountford J, Bisogrand M, Nolan $T$. Increasing Efficiency and Enhancing Value in Health Care - Ways to Achieve Savings in Operating Costs per Year [monografía en Internet]. Institute for Healthcare Improvement; 2009 [citado 15 ene 2011]. Disponible en: http://www. ihi.org/NR/rdonlyres/502ADCBF-878A. 4EAA-B191-21A7ADDE5357/0/1H IIncreasingEfficiencyand EnhancingValueinHealthCareWhitePaper2009.pdf

6. Repullo Labrador JR. Cambios y reformas en sistemas y servicios sanitarios. En: Gimeno JA, Rubio S, Tamayo P, eds. Sistemas y servicios sanitarios. Manuales de Dirección Médica y Gestión Clínica. Madrid: Ediciones Díaz de Santos; 2006:121-97.

7. Cosialls Pueyo D. Gestión clínica. Rev Adm San. 2000;16: 119.39.

8. Peiró S. Atención farmacéutica en el entomo hospitalario: la hora de las decisiones. Farm Hosp. 2006; 30:325-7.

9. López González AM, Sánchez Sánchez T. La gestión del conocimiento como herramienta clave para la mejora de las organizaciones sanitarias. Farm Hosp. 2007;31:3.4

10. Garcías-Altés A. La introducción de tecnologías en los sistemas sanitarios: del dicho al hecho. Gac Sanit. 2004;18:398-405.

11. Bernal Delgado E, Oliva J. Es necesario construir una cultura evaluativa en el Sistema Nacional de Salud. El médico. 2010;11: 10-2.

12. González Fuentes MV, García de la Serrana JL, Benavides Rosero C. El gasto farmacéutico en España [monografía en Internet]. Strategic Research Center Bussines School; 2010 [citado 20 ene 2011]. Disponible en: http://www.eae.es/ es/strategic-research-center.html

13. Consejo Interterritorial del Sistema Nacional de Salud. Acciones y medidas para promover la calídad, la equidad, la cohesión y la sostenibilidad del Sistema Nacional de Salud [monografía en Intemet] [citado 24 ente Politica /www.msps.es/ gabinetePrensa/discursos Interv/archivos/180310203430.pdf

14. Documento de posición AES sobre el uso de la evaluación económica en sanidad [monografía en Internet]. Asociación de Economia de la Salud (AES); 2008 [citado 25 ene 2011]. Disponible en: http://www.aes.es/Publicaciones/AESEE2.pd

15. Brosa M, Gisbert R, Rodríguez JM, Soto J. Principios, métodos y aplicaciones del análisis del impacto presupuestario en el sector sanitario. Pharmacoeconomics-spanish research articles. 2005;2:65-78.

16. Drummond MF, O'Brien BJ, Stodart GL, Torrance GW, eds. Métodos para la evaluación económica de los programas de asistencia sanitaria. Madrid: Ediciones Díaz de Santos; 2001

17. Catalá-López F, García-Altés A. Evaluación económica de intervenciones sanitarias en España durante el período 1983-2008. Rev Esp Salud Pública. 2010;84:353-69.

18. Sacristán JA, Rovira J, Ortún V, García-Alonso F, Prieto L, Antoñanzas $F$. Utilización de evaluaciones económicas de intervenciones sanitarias. Med Clin. 2004:122:789-95.

19. López Bastida J, Oliva J, Antoñanzas F, García-Altés A, Gisbert $\mathrm{R}$, Mar J, et at. Propuesta de guía para la evaluación económica aplicada a las tecnologías sanitarias. Madrid: Plan Nacional para el SNS del MSC. Servicio de Evaluación del Servicio Canario de la Salud; 2008. Informes de Evaluación de Tecnologías Sanitarias: SESCS N." 2006/22.

20. Diez Rodrigálvarez MV, Gómez Pajuelo P, Ortega Eslava $A$, Ausejo Segura M, Cruz Martos E, Rubio Terrés C. Integración y aplicaciones de los estudios de farmacoeconomia e investigación aplicaciones de los estudios de farmacoeconomia e investigacion Real Academia Nacional de Farmacia; 2001. Disponible en: Real Academia Nacional de Farmacia; 2001. Disponible
http://www. analesranf.com/ Acceso 27 de enero de 2011.

21. Soto Álvarez J. Estudios de farmacoeconomia, ¿por qué, cómo, cuándo y para qué? MEDIFAM. 2001;11:146-55.

22. Poveda Andrés JL, García Gómez C, Hernández Sansalvador M, Valladolid Walsh A. Análisis coste-beneficio de la implantación de los sistemas automáticos de dispensación de medicamentos en la Unidades de Críticos y Urgencias. Farm Hosp. 2003;27:14-21.

23. Cuesta Grueso C, Poveda Andrés JL, García Pellicer J, Romá Sánchez E. Análisis de minimización de costes de darbepoetin alfa frente a epoetina alfa en pacientes con insuficiencia renal crónica sometidos a hemodiálisis. Farm Hosp. 2010;34:68-75.

24. Romá.Sánchez E, Poveda Andrés Jl, García.Pellicer J, Salavert-Lletí M, Jarque-Ramos I. Estudio coste-efectividad de la estrategia empírica antifúngica en pacientes oncohematológicos. Farm Hosp. 2008;32:7-17.

25. Carrera-Hueso FJ. Aplicación de los estudios farmacoeconómicos en el hospital. Med Clín. 1998;111:347-53. 


\section{Capítulo 9}

Poquet Jornet JE, Carrera-Hueso FJ, Gasent Blesa JM, Peris Godoy M. Aspectos farmacoeconómicos de los citostáticos orales. Farm Hosp 2011; 35 (Supl 1): 25-31. 


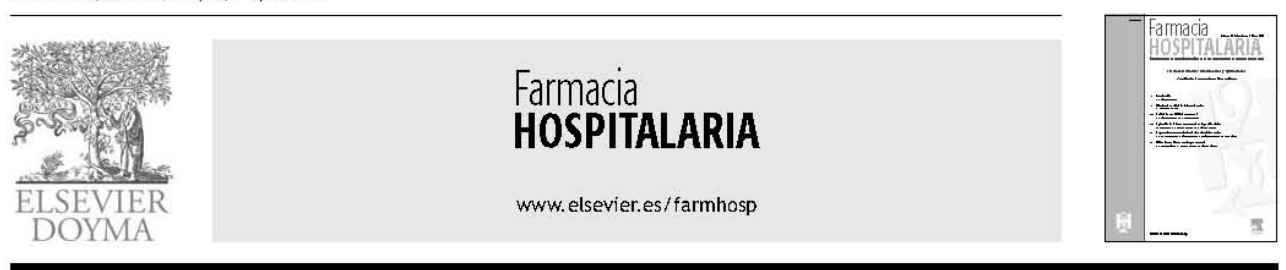

\title{
Aspectos farmacoeconómicos de los citostáticos orales
}

\section{J.E. Poquet Jornet ${ }^{a,{ }^{*}}$, F.J. Carrera-Hueso ${ }^{b}$, J.M Gasent Blesac y M. Peris Godoy}

\author{
aefe del Área Clínica de Farmacia, Hospital de Denia, Denia, Alicante, Espańa \\ - Servicio de Farmacia, Hospital Dr. Moliner, Serra, Valencia, España \\ 'Jefe del Área Clínica de Oncología, Hospital de Denia, Denia, Alicante, España \\ dDirector Asistencial, Hospital de Denia, Denia, Alicante, España
}

PALABRAS CLAVE

Quimioterapia oral;

Citostáticos;

Farmacoeconomía;

Cumplimiento;

Valor

\section{KEY WORDS}

Oral chemotherapy;

Cytostatic agents:

Pharmacoeconomics;

Compliance;

Value
Resumen En la validación de la quimioterapia oral, el farmacéutico debe comprobar la idoneidad de la prescripción, así como la corrección de su contenido, aplicando los mismos estándares de seguridad para los citostáticos parenterales. Cada vez hay más cánceres que tienen disponibles tratamientos por vía oral, mejorando la satisfacción del paciente, ya que pueden tomarse en sus domicilios sin necesidad de visitar el hospital. A medida que se incrementen los tratamientos citostáticos por vía oral será mucho más importante as egurarse de que ese cumplimiento de los tratamientos sea lo más óptimo posible. Los nuevos citostáticos por vía oral son menos tóxicos, reducen los costes indirectos e implican una menor pérdida de tiempo para el paciente y sus cuidadores, pero sus costes deberían estar por debajo de un umbral aceptado por la sociedad. Para ayudar a esta toma de decisiones, deberemos emplear las herramientas que la farmacoeconomía pone en nuestras manos.

(c) 2011 SEFH. Publicado por Elsevier España, S.L. Todos los derechos reservados.

\section{Pharmacoeconomic aspects of oral cytostatic agents}

Abstract When validating oral chemotherapy, pharmacists should confirm the suitability and correctness of the prescription, applying the same safety standards as those used for parenteral cytostatic drugs. There are an increasing number of cancers for which orally administered drugs are available, which increases patient satisfaction as these drugs can be taken at home without the need to visit a hospital. As oral cytostatic treatments increase, so does the importance of ensuring optimal treatment compliance. The new oral cytostatic agents are less toxic, reduce indirect costs and imply less loss of time for patients and their families. However, the cost of these agents should be below a threshold acceptable for society. As an aid to decision making, pharmacoeconomic tools should be used.

(1) 2011 SEFH. Published by Elsevier España, S.L. All rights reserved.

*Autor para correspondencia.

Correo electrónico: jaime.poquet@marinasalud.es (J.E. Poquet Jornet).

1130-6343/\$ - see front matter (O) 2011 SEFH. Publicado por Elsevier España, S.L. Todos los derechos reservados. 
Resultados y discusión

\section{Introducción}

La eficacia y la seguridad son los dos atributos básicos que de ben acompañar a cualquier tratamiento con medicamentos. $\mathrm{E}$ tratamiento con citostáticos es un caso particular en que habitualmente el paciente acude a un hospital a recibir su tratamiento, aunque este concepto ha evolucionado desde la introducción de los citostáticos orales.

El farmacéutico debe comprobar la idoneidad de la prescripción, así como la corrección de su contenido, aplicando los mismos estándares de seguridad en la validación de la quimioterapia oral que la que realiza para los citostáticos parenterales'. El empleo de quimioterapia oral no está libre de riesgos y una dosis mal entendida y mal administrada, diariamente, puede provocar reacciones graves en el paciente. En efecto, los citostáticos orales no son menos tóxicos que los tratamientos intravenosos, $y$ todos ellos presentan un estrecho intervalo terapéutico que ponen al paciente en riesgo de sufrir peligro sos efectos secundarios ${ }^{2,3}$.

Se incluyen como quimioterapia oral aquellos fármacos que tiene actividad antitumoral directa como capecitabina, ciclofosfamida, busulfán, hidroxicarbamida, idarrubicina, clorambucilo vinorelbina, tegafur, etopósido, topotecán, metotrexate y mel falán; o aquéllos que actúan dirigidos contra un receptor como imatinib, lapatinib, erlotinib, gefitinib, sunitinib, sorafenib y everolimus. No se incluyen los fármacos hormonales o antihormonales (tamoxifeno, anastrozol), mientras que sí se incluyen la talidomida y la lenalidomida4. Entre 2006 y 2007, sólo en Inglaterra se han prescrito más de 24 millones de dosis de citostáticos orales ${ }^{4}, y$ se espera que su número se incremente en los próximos años: de los 400 medicamentos que hay en investigación, prácticamente un $25 \%$ serán citostáticos orales s.5.5. $^{3.5}$

El tratamiento del cáncer supone el mayor campo de innovación en medicina, representando además un elevado impacto económico. En efecto, anualmente los costes de los antineoplásicos ascienden a $\mathbf{4 0 . 0 0 0}$ millones de dólares en todo el mundo $^{7}$. La Food and Drug Administration (FDA) ha aprobado 53 nuevas indicaciones en oncología entre los años 2005 y 2007, 18 de ellas correspondientes a nuevas moléculas. La FDA, en 2003, tenía en investigación 925 antineoplásicos, mientras que en 2008 esta cifra se había elevado a 1.440. Actualmente, las ventas de productos anticancerosos crecen a un ritmo superio a un $15 \%$ anual ${ }^{7,8,9}$. Más del $90 \%$ de los tratamientos antineoplásicos aprobados por la FDAen los últimos cuatro años tienen un coste de más de 20.000 dólares anuales 10 . A pesar del esfuerzo económico realizado, la tasa de mortalidad de la mayoría de cánceres ha descendido sólo modestamente y, comparativamente, mucho más lentamente que la mortalidad por patología cardiovascular. En EE. UU., los gastos sanitarios por habitante son más del doble de lo invertido en nuestro país $y$, sin embargo, no presentan unas supervivencias en el tratamiento del cáncer mucho mejores que las del resto de países europeos (Schickedanz, 2010).

Via oral frente a via intravenosa en la administración de citostáticos ${ }^{5-8,11-16}$

\section{Punto de vista del paciente}

Los pacientes pueden preferir la vía oral por las siguientes razones:
- Mayor comodidad en su administración

- Evitar desplazamientos al hospital de día

- Evitar el tiempo necesario para la administración de la quimioterapia IV

- Disminuir la ansiedad en los pacientes

- Cuando el acceso venoso no es accesible

- Menor riesgo de complicaciones

Los pacientes pueden preferir la vía intravenosa cuando:

- Le presuponen una mayor eficacia a la vía intravenosa

- El número total de comprimidos supera los 6-8 por día

- El paciente debe organizar su vida alrededor de la inges. ta de la medicación, por ejemplo, el tegafur más uracilo debe tomarse tres veces al día y separado una hora de las comidas

- La administración por vía oral va acompañada de un mayor control, como en la administración de vinorelbina; el elevado riesgo de neutropenia grado 3.4 hace que se re. quieran analíticas semanales

\section{Punto de vista del oncólogo}

Los facultativos pueden preferir la vía oral por:

- Todas las razones que le convienen al paciente y que han sido citadas anteriormente

- Para solucionar los problemas de escasez de personal, ya que sustituir la quimioterapia intravenosa por oral permitiría incrementar en siete veces el número de pacientes tratados ${ }^{17}$. Cambiar del esquema de Gramont por capecitabina oral en pacientes con cáncer colorrectal avanzado permitiría ahorrar más de 10 horas por paciente en visi. tas médicas, lo que permitiría que por cada 50 pacientes con 5 -fluorouracilo intravenoso se podrían tratar otros 350 pacientes con capecitabina oral ${ }^{18}$

Los facultativos pueden preferir la vía intravenosa cuando:

- Asumen una mayor eficacia que la vía ora

- Problemas sobre la biodisponibilidad de los fármacos:

a) La presencia de glicoproteína $P$ en el epitelio intestinal impide la absorción de varios fármacos (por ejem. plo: paclitaxel oral) ${ }^{19}$.

b) Varios fármacos presentan escasa biodisponibilidad cuando se administran por vía oral: vinorelbina (33\%), topotecán (35\%) y etopósido $(40-75 \%)$

c) Elevada variabilidad en la biodisponibilidad de topotecán provoca una mayor incidencia de neutropenia cuando se da la dosis máxima por vía oral frente a la administración intravenosa.

d) La biodisponibilidad de los citostáticos orales puede verse influida por la presencia de comida (tegafur).

e) Interacciones con otros medicamentos ${ }^{8,18}$. En pacientes con gliobastoma, la administración de un antiepiléptico inductor hepático requiere la administración de $500 \mathrm{mg}$ de imatinib cada 12 horas, mientras que, si no se administra el antiepiléptico, es suficiente con $400 \mathrm{mg}$ diarios. La administración conjunta de erlotinib con inhibidores (ciprofloxacino, itraconazol) 0 in ductores (fenitoína, carbamacepina o fenobarbital) 
Resultados y discusión

del CYP3A4 e inhibidores de la bomba de protones (que al alterar el pH del tracto digestivo pueden modificar la biodisponibilidad y la solubilidad del erlotinib provocando toxicidades inesperadas o importantes pérdidas de eficacia) ${ }^{6}$

f) No tienen la seguridad del cumplimiento correcto y la vía intravenosa proporciona una mayor oportunidad de monitorizar el tratamiento. Un $\mathbf{4 3} \%$ de los pacientes con cáncer de mama no tomaba el citostático ora tal como había sido prescrito ${ }^{20}$. Con los citostáticos orales se pueden continuar presentando sobredosifica-
ciones incluso en presencia de toxicidades que pongan en riesgo la vida del paciente, por lo que es imprescindible realizar un ajuste de dosis individualizado para el manejo adecuado de los efectos adversos.

g) Por las dificultades asociadas a poblaciones especiales que presenten síndrome de malabsorción, disfunciones hepáticas, alteraciones orofaríngeas u obstrucción intestinal.

h) Por el sistema de pago al oncólogo: en EE. UU., Medicare paga al oncólogo por la administración de quimioterapia intravenosa, pero no cubre la administración de citostáticos orales, por lo que su remuneración económica es muy superior si administran quimioterapia intravenosa que si la administran por vía oral ${ }^{6}$. En la última década los salarios de los oncólogos (al menos los de EE. UU.) se han incrementado en un $86 \%$ cuando su actividad ha crecido un $12 \%$ ? Actualmente, Medicare abona al oncólogo un precio medio más un $6 \%$ del precio del fármaco, lo que crea un "incentivo perverso", pues cuanto más caros sean los tratamientos prescritos, mayor es el margen y, por lo tanto, el beneficio económico. La mediana anual del salario de los oncólogos, en EE. UU. es de 358.000 dólares, siendo la media de 523.000 . El $10 \%$ de los oncólogos ganaban, en el 2006, más de un $1 \mathrm{mi}$ llón de dólares anuales ${ }^{10}$.

\section{Errores de medicación y cumplimiento con citostáticos orales}

Entre 1993 y 2002 se han descrito 25 fallecimientos y 26 efectos adversos graves asociados con errores en la prescripción de metotrexate oral, debido a que los pacientes no recibieron suficiente información o ésta fue inadecuada ${ }^{3}$. Se han descrito tres muertes y más de 400 errores relacionados con la administración de citostáticos orales entre los años 2003 y 2007; la mitad de los cuales estaban relacionados con una dosis, una frecuencia o una duración de tratamiento inadecuada 4 .

En caso de producirse un error en la prescripción, valida ción, dispensación o de comprensión por parte de un paciente de un medicamento citostático oral, éste no será detectado hasta la próxima dispensación o la próxima vista médica. Por tanto, es importante seguir una serie de recomendaciones dirigidas a disminuir el error y facilitar la comprensión del paciente $o$ familiar ${ }^{2}$ :

a) Describir las dosis de las diferentes tomas: indicar nú mero de formas farmacéuticas que deben ser tomadas por dosis, evitando especificar únicamente la dosis diaria total.

b) Identificar el número de dosis que deben tomarse, identificando el número de tomas al día y sin dejar duda a la confusión.

c) Ajustar la dosis a la presentación disponible de las formas orales sólidas: por ejemplo, lomustina $130 \mathrm{mg} / \mathrm{m}^{2}$ dosis prescrita $=208 \mathrm{mg}$, entonces dispensar lomustina $200 \mathrm{mg}$ equivalente a 5 cápsulas.

d) En relación con las comidas: por ejemplo cuando se prescribe erlotinib $150 \mathrm{mg}$ una vez al día, cada día durante 30 días, se debe indicar: tomar separado al menos dos horas, de las comidas.

e) Medicación de soporte y concomitante (temozolomida y antieméticos).

f) Confirmación de la comprensión por parte del paciente en la dispensación.

La existencia de personal especializado que realice al principio de cada tratamiento con citostáticos orales una sesión personalizada de 60-90 minutos, la entrega de calendarios que recuerden cómo administrar correctamente la medicación, hacer que el paciente conozca y comprenda cuales son los efectos adversos que se pueden presentary disponer de kits que les ayuden a controlar sus efectos adversos han demostrado que son estrategias que mejoran el cumplimiento con los citostáticos orales y, por lo tanto, los resultados clínicos asociados ${ }^{3 \cdot 5,20,21}$. A medida que se incre. menten los tratamientos citostáticos por vía oral será mucho más importante asegurarse que ese cumplimiento de los tratamientos sea lo más óptimo posible ${ }^{11}$.

La eficacia, y también la toxicidad, de los citostáticos orales dependen de la adherencia adecuada a la dosis pres. crita, siendo el paciente el último responsable de garantizar un cumplimiento óptimo ${ }^{6}$. Entre las razones que se han des crito para justificar este bajo cumplimento destacan las si guientes: sentir que se está mejor y dejar de tomar el fármaco; sentir que se está peor y dejar de tomar el fármaco; la dosificación del citostático oral es complicada y confusa; incapacidad para financiar medicamentos caros ${ }^{15}$.

En ocasiones, la aparición de efectos adversos, más allá de lo que es tolerable o seguro, puede hacer que el paciente deje de tomarse la medicación. Estas "vacaciones terapéuticas" pueden incrementar los costes asociados al tratamiento por la medicación desperdiciada, por la pérdida de los avances obtenidos con la terapia, por la progresión de la enfermedad y por una utilización adicional de servicios sanitarios a medida que se deteriora la salud del paciente. La quimioterapia oral también puede producir efectos ad versos importantes que, al no ser detectados hasta la próxima recogida de medicación o la visita con el oncólogo pueden afectar a la situación clínica del paciente ${ }^{11}$.

Asegurar el cumplimiento de la quimioterapia oral es difícil al tomarse en el domicilio del propio paciente, y sólo se puede monitorizar en función de lo que el paciente notifica, lo que supone no poder aplicar mediciones externas como el recuento de comprimidos ${ }^{6}$ o por el efecto Hawthorne ${ }^{15}$.

A pesar de conocer el hecho de que la supervivencia de paciente y la mejora de su situación clínica dependen de una ingesta adecuada de la medicación oral, las sobredosificaciones, las infradosificaciones y los errores en la admí. nistración de la quimioterapia oral (por ejemplo: 
Resultados y discusión

presencia/ausencia de comidas o administración en el momento inadecuado) pueden hacer que hasta el $80 \%$ de los pacientes no se tomen los citostáticos orales tal como se los ha prescrito el oncólogo. Entendiéndose cumplimiento terapéutico a tomarse la medicación, tal como ha sido prescrita, en más de un $85 \%$ de las tomas. Entre los factores que elevan el riesgo de no cumplimiento destacan ${ }^{11}$ : a) complejidad del régimen terapéutico; b) la edad del paciente (se han descrito especiales problemas de cumplimiento en los adolescentes); c) las expectativas del paciente sobre la efectividad del tratamiento; d) la gravedad de la enfermedad y el cambio clínico esperado; e) la "educación sanitaria" que tengan el paciente y su familia; f) una escasa supervisión de la adherencia por parte de los profesionales del hospital.

Una preocupación expresada por los oncólogos es que los pacientes infranotifiquen los efectos adversos asociados con la administración de citostáticos orales. En efecto, en un estudio se ha detectado que un $23 \%$ de los pacientes que habían tenido un efecto adverso no lo habían notificado porque pensaban que la quimioterapia oral tiene que estar asociada a una mejor tolerancia o porque temen que s comunican el efecto adverso se suspenderá la quimioterapia, con el riesgo de avance del tumor que ello suponet. Los sistemas de contaje de comprimidos no aseguran la ingesta adecuada de los citostáticos orales ${ }^{6}$.

Entre los métodos descritos para mejorar el cumplimiento de la quimioterapia oral destacan las llamadas telefónicas o recordatorios electrónicos, incrementar la educación de los pacientes y de sus cuidadores y un manejo adecuado de los efectos adversos asociados al tratamiento ${ }^{15,22}$

\section{Estudios farmacoeconómicos}

En los ensayos clínicos de medicamentos citostáticos, el in cremento de la supervivencia es generalmente el principal objetivo. Sin embargo, en la práctica clínica estos resultados de supervivencia se suelen emplear para la selección de tratamiento, mientras que la decisión clínica de continuar con las mismas dosis, reducir la dosis de quimioterapia administrada o incluso suspender los tratamientos están relacionados con la aparición de los efectos adversos, y éstos pueden evaluarse con escalas validadas de la calidad de vida de los pacientes. La administración oral de citostáticos, en principio, está asociada con una menor tasa de efectos adversos que la vía intravenosa, con la mejora que ello supone para la calidad de vida, y pueden reducir los costes globales del sistema sanitario ${ }^{12}$

En un estudio de pacientes con cánceres incurables y que recibían quimioterapia paliativa, el $89 \%$ alegaron que preferirían la vía oral a la intravenosa. Sin embargo, el 70\% alegaron que no aceptarían una menor tasa de respuesta y un $74 \%$ dijeron que no aceptarian una menor duración de la respuesta por el hecho de emplear la vía oral. Como era de prever, la eficacia del tratamiento en este tipo de pacientes está por encima de la comodidad de la administración del tratamiento ${ }^{23}$.

En cáncer colorrectal metastático (CCRM), la administración de capecitabina oral generó menores estancias hospitalarias para recibir la quimioterapia, menores días de hospitalización ocasionados por los efectos adversos y una menor necesidad de fármacos para tratar efectos adversos (utilizando fluconazol y antagonistas del $5-\mathrm{HT}_{3}$ ). En un estudio francés en pacientes con cáncer de colon estadio III, la administración de capecitabina generó unos ahorros de 7.025 dólares por paciente cuando se comparaba con el protocolo de la Clínica Mayo y de 3.569 dólares cuando se comparaba con el protocolo de Gramont ${ }^{24}$. En un estudio italiano también se demostraba que la capecitabina era la estrategia dominante, generando unos ahorros de 2.234 euros por paciente ${ }^{25}$.

Capecitabina ha demostrado ser más coste-efectiva que la administración intravenosa de 5-FU, de gemcitabina y de vinorelbina ${ }^{5}$. Un análisis farmacoeconómico de capecitabina oral frente a 5 -fluorouracilo más leucovorin en pacientes con cáncer de colon grado $\mathrm{C}$ de Dukes, aunque los costes de adquisición fueron superiores para capecitabina (2.081 frente a 602 libras), los mayores costes asociados con la administración de la quimioterapia intravenosa (los pacientes con 5FU/LV necesitaron 28 visitas frente a las 7,4 visitas de los pacientes con capecitabina), el empleo de fármacos más económicos para tratar los efectos adversos asociados con la administración de quimioterapia y el menor tiempo empleado en visitas ( 27 frente a 125 horas) permitieron obtener unos costes un $57 \%$ inferiores con la administración de capecitabina. Los ahorros estimados serían de 3.700 libras por paciente, que se elevan a 5.000 libras si se incluyen los costes asociados al desplazamiento del paciente ( 3.357 con capecitabina frente a 8.528 libras con 5FU/LV). La supervivencia a 36 meses fue del $81,3 \%$ con capecitabina y de $77,6 \%$ con $5 F U / L V$ (diferencia absoluta del $3,7 \%$ ) lo que hace que el tratamiento con capecitabina sea la opción dominante ${ }^{26}$.

En un estudio en el que se analizaba el coste del tratamiento de cisplatino más capecitabina frente a cisplatino más fluorouracilo en pacientes con cáncer gástrico, los costes en la rama con capecitabina fueron de 2.688 dólares por paciente y de 4.014 dólares en los pacientes tratados en el brazo de fluorouracilo ${ }^{27}$.

Se analizó la utilización de capecitabina en cáncer colorrectal metastásico (CCRM) (bien en monoterapia, bien asociado a oxaliplatino), en monoterapia en cáncer de mama metastásico (CMM) y el uso de erlotinib en monoterapia en cáncer de pulmón no microcítico metastásico (CPNM), y se compararon los costes asociados con los obtenidos con la quimioterapia intravenosa habitual. El coste asociado al tratamiento intravenoso fue el siguiente: a) CCRM: 20.200 euros y 11.258 euros con capecitabina (ahorro de 8.943 euros por paciente); b) CMM: 13.775 euros y 4.703 euros con capecita. bina (ahorro de 9.072 euros por paciente); y c) CPNM: 12.405 euros y 10.141 euros con erlotinib (ahorro de 2.264 euros por paciente) $)^{28}$.

En pacientes con cáncer de mama metastásico, la administración de capecitabina tenía unos costes mensuales por paciente inferiores a los de vinorelbina $(7.032$ frente a 9.660 dólares), a los de gemcitabina ( 6.930 frente a 12.716 dólares), a los de taxanos más antraciclina (10.895 frente a 13.115 dólares) y frente a otros protocolos con taxanos ( 9.253 frente a 12.116 dólares). Estos menores costes están relacionados con una menor tasa de efectos adversos (mielosupresión y eventos gastrointestinales) y por los menores costes asociados con la administración de capecitabina $^{29}$ (Rugo). Se ha realizado un estudio en que a los 
Resultados y discusión

pacientes HER + y resistentes al trastuzumab que recibieron capecitabina y lapatinib, observando que el coste de añadir el lapatinib a la capecitabina fue de 10.630 dólares con una ganancia esperada de 0,12 años ajustados por calidad (AVAC), obteniendo un coste-efectividad incremental de 166.113 dólares por AVAC, por lo que la adición de lapatinib en estas condiciones, claramente, no es una opción costeefectiva ${ }^{29}$.

En un estudio coste-beneficio realizado en pacientes con cáncer colorrectal, la administración de capecitabina oral frente al protocolo de la Clínica Mayo generó unos ahorros por paciente de 1.610 euros, si se empleaba en primera línea, y de 943 euros si se empleaba en adyudancia ${ }^{30}$. Los be neficios clínicos deben ser expresados como términos absolutos más que como términos relativos, ya que ello evita sobrestimaciones del beneficio clínico. Además, estas mediciones de beneficios clínicos deben ser transparente y fáciles de interpretar ${ }^{9}$

En pacientes con cáncer de pulmón no microcítico, se ha realizado un estudio de impacto presupuestario en el que se simulaba el tratamiento de 50.000 pacientes. Como tratamiento de segunda línea, se comparaba erlotinib frente a docetaxel y permetrexed ( $30 \%$ de los pacientes reciben erlotinib), y en tercera línea se comparaba erlonitinib frente a tratamiento paliativo ( $90 \%$ de los pacientes reciben erlotinib), obteniendo que los costes para un año de tratamiento era de 382.418 dólares para los pacientes que recibieron erlotinib y de 380.968 para los pacientes sin erlotinib (diferencia de 1.450 dólares) ${ }^{31}$

Entre las limitaciones de los estudios de coste efectividad realizados con citostáticos orales hay que destacar que pueden estar basados en el análisis de grandes bases de datos, que se pueden haber realizado en diferentes periodos de tiempo y que se presenten diferencias importantes en el diseño de los estudios y en los criterios de selección de las poblaciones analizadas ${ }^{25}$.

En un estudio realizado en pacientes con cáncer de colon metastásico, los pacientes que recibieron la quimioterapia en su domicilio recibieron 5,3 ciclos, mientras que los pacientes que la recibieron en la consulta privada del oncólogo recibieron 9,0 ciclos. Es fácil imaginar el impacto que tiene donde se recogen los datos de consumos sobre los cos. tes en un estudio de farmacoeconomía ${ }^{32}$. En ciertas ocasiones, el coste del tratamiento está influenciado por la indicación para la que se emplea; así, lenalidomida, que tenía un coste de 55.000 dólares por paciente/año cuando se empleaba como tratamiento de la anemia asociada a síndrome mielodisplástico ( $10 \mathrm{mg} /$ día), elevó su precio hasta 74.000 dólares por paciente/año en cuanto se aprobó su empleo en mieloma múltiple ( $25 \mathrm{mg} /$ día) (Curtiss, 2006). Las conclusiones de diferentes estudios farmacoeconómicos pueden diferir entre las diversas agencias evaluadoras. Además, hay que reevaluar el nuevo impacto económico ante la ampliación de las indicaciones clínicas para las que se emplea el fármaco ${ }^{33}$

\section{El valor de los citostáticos orales}

Para la mayoría de bienes y servicios, la competencia de los mercados ajusta el valor que los consumidores dan a las cosas con el coste de producción de los fabricantes. Si los con- sumidores están bien informados y les pertenece el dinero que gastan, los precios que ellos están dispuestos a pagar se acaban acercando al valor del producto, al tiempo que la competencia obliga a los fabricantes a ajustar este precio a los costes de fabricación. Sin embargo, en salud está mucho menos claro lo que es valor, precio y coste. A los pacientes, a los que financian la sanidad e incluso a los médicos, en ocasiones les falta información sobre la efectividad y riesgos, especialmente con los nuevos medicamentos. Además, las patentes limitan la competencia y reducen la presión competitiva sobre el precio de los productos ${ }^{34}$. En el año 2000 , en EE. UU., se emplearon 128.000 millones de dólares en medicamentos (incremento del $13,6 \%$ respecto al año anterior); de esa subida en el gasto, un $3,9 \%$ fue ocasionado por incrementos de precios y un $9,7 \%$ por un incremento en su utilización ${ }^{35}$.

Uno de los asuntos más espinosos en medicina es el concepto de qué es lo que constituye valor en salud, ya que conocer y acotar este concepto nos llevará a conocer dónde y cómo estamos empleando nuestros recursos ${ }^{\circ}$. El término valor en cáncer lo podríamos definir como aquel tratamiento o procedimiento que prologa la vida del paciente $\mathrm{y} / \mathrm{o} \mathrm{me}$ jora su calidad de vida con unos costes por AVAC razonables para la sociedad ${ }^{36}$

En una reunión de consenso, organizada por el Instituto de Medicina en EE. UU., y que reunió a oncólogos, investigadores, pacientes, industria farmacéutica, economistas y gestores para definir qué es lo que tiene valor en el tratamiento del cáncer, se ha concluido que está constituido por diversas dimensiones como son: resultados, cuidados, pacientes y costes. Y que la medición de la dimensión económica debe realizarse con costes por $A V A C$, es decir, dólares o euros/AVAC? . La comparación de los costes por AVAC proporciona una medida razonable para medir el valor obtenido por cada dólar gastado y proporciona un método estandarizado para evaluar costes y resultados. Al analizar fármacos para el cáncer frente a fármacos utilizados en otro tipo de indicaciones se han obtenido mayores valores de coste-utilidad (media, mediana y mínimo) con los antineoplásicos $(68.000,71.000$ y 63.000 dólares) frente a los que no lo son $(63.037,63.000 \text { y } 9.225 \text { dólares })^{34}$.

En un momento de recursos limitados y de un incremento incesante de los costes de los nuevos tratamientos oncológi$\cos$, es razonable considerar qué es lo que aporta valor tanto para el paciente como para la sociedad cuando se toman decisiones en el tratamiento de los pacientes oncológicos?. Aunque los nuevos tratamientos pueden ofrecer beneficios en salud, sus elevados precios plantean si los recursos que destinamos a su adquisición no podrían invertirse en otras intervenciones sanitarias con un ratio coste-efectividad más favorable. En efecto, para el Instituto Británico para la Salud y Excelencia Clínica (NICE) aquellas con un coste-efectividad inferior a 40.000 euros serían rentables, aunque recientemente NICE ha relajado este límite para aquellos cánceres poco frecuentes que generarán un limitado impacto en las cuentas del National Health Service $(\mathrm{NHS})^{36}$, si hay que plantearse la sostenibilidad del sistema sanitario o el copago por parte de los pacientes y, sobre todo, si los beneficios obtenidos compensan los elevados costes asociados con su administración ${ }^{34}$. En cuanto a la evaluación de los costes, la introducción de fórmulas de copago, cuando el paciente no abona más de un $20 \%$ del valor del fármaco, no 
Resultados y discusión

promociona el uso de los tratamiento con mayor "utilidad terapéutica", sino tan sólo de los de "menor coste"?

El vertiginoso incremento de los costes de la quimiotera pia en los últimos tiempos nos hace ser más exigentes al analizar si se ha incrementado del mismo modo el valor de los resultados clínicos obtenidos. Aunque los costes/AVAC de la mayoría de nuevos antineoplásicos son difíciles de calcular, con frecuencia exceden los 100.000 dólares/AVAC. Por ejemplo, para bevacizumab en cáncer colorrectal metastá. sico o cáncer de mama los costes oscilan entre 200.000 y 300.000 dólares/AVAC, y por tanto el NICE ha recomendado que no sea financiado por el NHS. Recientemente, un alto ejecutivo de la industria farmacéutica ha reconocido que de las nuevas moléculas que están a punto de comercializar "ninguna de ellas está por debajo de 300.000 dólares/AVAC" 36 .

A los oncólogos se les pide que tienen que ponderar si los beneficios clínicos esperados superan los costes del tratamiento, pero incorporar estas decisiones a su práctica clínica no suele ser muy de su agrado (el $88 \%$ de los oncólogos creen que el coste de los tratamientos no debería influir en sus decisiones terapéuticas y consideran que un beneficio clínico de un par de meses ya justificaría un coste-efectividad de 300.000 dólares/AVAC ${ }^{7}$ ). Los pacientes deben elegir entre cantidad y calidad de vida ${ }^{37}$. Hasta un $40 \%$ de los pacientes pueden estas preocupados por los costes del tratamiento oncológico, aunque la mayoría de ellos no transmiten nunca sus inquietudes a su médico ${ }^{7}$.

Se ha analizado el impacto de la introducción de nuevos fármacos en el tratamiento del cáncer de colon metastási co, en dólares, durante ocho semanas (tabla 1). En el numerador de la ecuación coste-beneficio sin quimioterapia la supervivencia media es de 8 meses, de 12 meses con fluorouracilo y 21 meses con FOLFIRI o FOLFOX. La adición de bebacizumab o cetuximab incrementa el coste medio por paciente hasta 161.000 dólares, sin ninguna evidencia de un incremento de la supervivencia por encima de los 21 meses obtenidos con FOLFIRI o con FOLFOX ${ }^{13}$.

Muchos de los nuevos antineoplásicos tienen unos costes superiores a 25.000 dólares por año, mientras que los beneficios, en los mejores casos, tan sólo alcanzan para unos pocos meses. En un estudio realizado con 139 oncólogos de EE. UU., sólo el $30 \%$ reconocía que el coste de los tratamientos influía en sus decisiones terapéuticas, aunque un $71 \%$ creía que durante los próximos años se iba a incremen-

\begin{tabular}{|c|c|}
\hline Protocolo & Coste $(\$$ \\
\hline Clínica Mayo: bolus mensual de fluorouracilo & 63 \\
\hline $\begin{array}{l}\text { Roswell Park: bolus semanal de fluorouracilo } \\
\text { más leucovorin }\end{array}$ & 304 \\
\hline $\begin{array}{l}\text { LVSFU2: administración bisemanal de bolus } \\
\text { de } 48 \mathrm{~h}\end{array}$ & 363 \\
\hline FOLFIRI (LVSFU2 + irinotecan) & 9.831 \\
\hline FOLFOX (LVSFU2 + oxaliplatino) & 11.889 \\
\hline Bebacizumab + (FOLFIRI o FOLFOIX) & 21.000 \\
\hline Cetuximab + FOLFIRI & 30.700 \\
\hline
\end{tabular}

tar el control sobre el coste económico de los tratamientos. Un $62 \%$ justificaba unos incrementos de 70.000 dólares anuales sobre el tratamiento de referencia, si se obtenia un beneficio adicional de sólo $2-4$ meses, mientras que otro $20 \%$ adicional creía que para justificar este importe hacía falta un incremento de la supervivencia, como mínimo, de de 4-6 meses. El bevacizumab tiene un coste de 4.400 dólares con un beneficio medio en supervivencia de 4,7 me$\operatorname{ses}^{38}$.

Los pacientes con citostáticos orales pueden presentar incumplimientos muy elevados; en esos casos, los costes de la quimioterapia oral al realizar el estudio farmacoeconómico, ¿deben basarse en la adherencia real o en la adherencia prescrita ínicialmente? Por otro lado, en evaluaciones económicas, los costes y beneficios se extrapolan a una duración de varios años; si la molécula original pierde su patente y se comercializan genéricos con una disminución de su precio entre el 25 y el $75 \%$, ilos estudios farmacoeconómicos deben anticipar estos cambios futuros de precios? ${ }^{39}$. Es un error asumir en los estudios coste-efectividad que el valor de la vida es lineal o si los AVAC deben tener diferente valor en función de la edad del paciente ${ }^{36}$. Si, por muy elevados que sean los costes/AVAC de los nuevos antineoplásicos, nunca quedan fuera de la financiación pública, ¿por qué tendría que adaptarse la industria farmacéutica a buscar fármacos que no superasen un determinado umbral? ${ }^{36}$.

El tratamiento con quimioterapia en pacientes terminales nos obliga a plantearnos hasta cuándo debemos administrar quimioterapia cuando las expectativas de vida son mínimas. Entre el 16 y el $20 \%$ de los tumores sólidos en EE. UU., estuvieron recibiendo quimioterapia en los 14 días previos a su fallecimiento ${ }^{10}$. En algunas ocasiones, se incrementarían los AVAC obtenidos si estos elevados recursos económicos se empleasen en cribaje de cáncer en lugar de emplearlos en pacientes terminales en los que el beneficio clínico, en ocasiones, tan sólo supone unos meses, o incluso semanas, adicionales de vida (y además, con una escasa calidad de vida) ${ }^{35}$.

Sólo hay dos caminos para reducir el coste de los medicamentos proporcionados a los pacientes: o se reducen los medicamentos administrados o se paga menos por ellos ${ }^{10}$. Los nuevos citostáticos administrados por vía oral son menos tóxicos, reducen los costes indirectos, incluidos los costes de desplazamiento e implican una menor pérdida de tiempo para el paciente y sus cuidadores ${ }^{16}$, pero sus costes deberían estar por debajo de un umbral (medido en coste/AVAC) que la sociedad aceptase gastarse en su adquisición, así como no dedicar esos recursos económicos a otras actividades.

\section{Conflicto de intereses}

Los autores declaran no tener ningún conflicto de intereses, actual o pasado, con lo que se expone en el presente ar. tículo ni con los laboratorios que comercializan los medicamentos mencionados en el mismo.

\section{Bibliografia}

1. Cuesta Grueso C, Munilla Das A, Poquet Jomet JE. El farmacéu. tico especialista del área oncohematológica en el tratamiento 
Resultados y discusión

de pacientes con quimioterapia oral. Aten Farm. 2010;12: 203.4.

2. Pontón JL. Procedimientos de seguridad en el tratamiento con medicamentos citostáticos. Vol 1.3. Formación continuada para farmacéuticos de hospital. Módulo IV. Lab Ferrer. Sociedad Es pañola de Farmacia Hospitalaria.

3. Moody M, Jackowski J. Are patients on oral chemotherapy in your practice setting safe? Clin J Oncol Nurs. 2010;14:339-46.

4. Oakley C, Crowe M, Johnson M. Introducing the United Kingdom oncology nursing Society (UKONS) position statement on oral chemotherapy. Eur J Cancer. 2010;19:1-4.

5. Findlay $M$, Von Minckwitz G, Wardley A. Effective oral chemotherapy for breast cancer: pillars of strength. Ann Oncol. 2008; 19:212.22

6. Banna GL, Collova E, Gebbia V, et al. Anticancer oral therapy: emerging related issues. Cancer Treat Review. 2010;36: 595.605.

7. Schickedanz $A$. Of value: a discussion of cost, communication and evidence to improve cancer care. The Oncologist. 2010;15 (suppl 1):73-9.

8. Di Mario, Ratain MJ. Oral chemotherapy: rationale and future directions. J Clinical Oncology. 1998;16:2557-67.

9. Ramsey S, Schickedanz A. How should we define value in cancer care? The Oncologist. 2010;15 (suppl 1):1-4.

0. Smith TJ, Hillner BE. Concrete options and ideas for increasing value in oncology care: the view from one trench. The Oncolo gist. 2010;15 (suppl 1):65-72.

11. Reese DW, Skyberg JT, Kuntz GA. Oral oncology treatment regimens and the role of medication therapy management on patient adherence and compliance [consultado 12/01/2011] Disponible en: http://www, intellogyhealth.com/pdf/Oral Oisponible en:

12. Gralla RJ. Quality of life considerations in patients with ad vanced lung cancer: effect of topotecan on symptoms pallia. tion and quality of life. The Oncologist. 2004;9 (suppl 6):14-24

13. Curtiss FR. Pharmacy benefits spending on oral chemotherapy drugs. J Managed Care. 2006:12:570-7.

14. Peys F. Pharmacoecomics: where is the link with pharmacokinetics and biophamaceutics? Pharm World Sci. 1997;19:73-81.

15. Barefoot J, Blecher CS, Emery R. Keeping pace with oral chemo therapy. Oncology. 2009;24:36.9.

16. Sparreboom A, De Jonge MJA, Verweij J. The use of oral cyto toxic and cytostatic drugs in cancer treatment. Eur J Cancer 2002;38:18-22.

17. James $R, B$ lanco $C$, Farina $C$. Savings in staff time as a result of switching from de Gramont to oral capecitabina for patients with colorectal cancer. Eur J Cancer. 2003;1 (suppl 5):583 (abstract 271).

18. Irshad S, Maisey N. Considerations when choosing oral chemotherapy: identifying and responding to patient need. Eur J Cancer Care. 2010;19:5-11.

19. Bebawy M, Sze DM. Targeting P-glycoprotein for effective ora anti-cancer chemotherapeutics. Current Cancer Drug Targets. 2008;8:47-52.

20. Lebovits AH, Strain JJ, Schleifer SJ, Tanaka JS, Bhardwaj S, Messe MR. Patient non compliance with self-administered chemotherapy. Cancer. 1990;65:17-22.

21. So J. Improving the quality of oral chemotherapy services using hame care. Eur J Cancer. 2010;19:35.9.
22. Vidall C. Providing community oral chemotherapy services. Eur J Cancer Care. 2010;19:29-34.

23. Liu G, Franssen E, Fitch MI, Warner E. Patient preferences for oral versus intravenous palliative chemotherapy. J Clin Oncol. 1997; 15:110-5.

24. Husseini F, Douillard JY, Ychou M, Perrocheau G, Tilleul P, Maes $\mathrm{P}$, et al. Capecitabine vs. Mayo Clinic and the Gramont 5FU/LV regimens for stage III colon cancer: cost-effectiveness analysis in the French setting. Ann Oncol. 2006;17 (suppl 6):64 (abstract 133).

25. Di Constanzo F, Sobrero A, Twelves C, Douillard J, Giuliani G, Patel K. Italian economic evaluation of capecitabine vs bolus $5 \mathrm{FU} / \mathrm{LV}$ as adjundant chemotherapy for patients with Dukes' $C$ colon cancer. Ann Oncol. 2006;17 (suppl 6):63 (abstract 130).

26. Cassidy J, Douillard JY, Twelves C, McKendrick JJ, Scheithauer W, Bustová I, et al. Pharmacoeconomic analysis of adjudant oral capecitabine vs. intravenous 5.FU/LV in Dukes' $\mathrm{C}$ colon cancer: the X-ACT trial. British J Cancer. 2006;94:1122-9.

27. Darba J, Restovic G, Ramírez de Arellano A. Economic assess ment of capecitabin/cisplatin versus 5-FU/cisplatin regimens in treatment of advanced gastric cancer in Spain. 33th congress of the European Society form Medical Oncology. Stockholm, Suethe European Society form Medical

28. De Benito A, Caballo B, Gómez J, Moyano N, Giménez E, Prior $\mathrm{M}$, et al. Estudio comparativo de costes del tratamiento oral con Xeloda y Tarceva frente al tratamiento quimioterápico intravenoso desde la perspectiva de una aseguradora privada española. Rev Esp Econ Salud. 2010;9:28-36.

29. Rugo HS. Pharmacoeconomic considerations associated with use of oral agents in metastatic breast cancer [consultado 15/01/2011]. Disponible en: http://www.staging.medscape. org/viewarticle/710916

30. Jansman FG, Postma MJ, Van Hartskamp D, Willemse PH, Brou wers JR. Cost-benefit analysis of capecitabine versus 5 -fluorou$\mathrm{racil} /$ leucovorin in the treatment of colorectal cancer in the Netherlands. Clin Ther. 2004;26:579-89.

31. Carlson JJ. Erlotinib in non-small-cell lung cancer: a review of the clinical and economic evidence. Expert Rev Pharmacoeco. nomics Outcomes Res. 2009;9:409.16.

32. Newcomer $L N$. The responsibility to pay for cancer treatments: a health insurer's view of value. The Oncologist. 2010;15 (suppl 1):32.5.

33. Tilson L, O'Leary A, Usher C, Usher C, Barry M. Pharmacoeconomic evaluation in Ireland. Pharmacoeconomics. 2010;28: 307.22.

34. Danzon PM, Taylor E. Drug pricing and value in oncology. The Oncologist. 2010;15 (suppl 1):24.31.

35. Ingramm RA. Counting the true cost and value of medicines. The Oncologist. 2003;8:2-4.

36. Brock DW. Ethical and value issues in insurance coverage for cancer treatment. The Oncologist. 2010;15 (suppl 1):36-42.

37. Ubel PA. Beyond cost and benefits: understanding how patients make health care decisions. The Oncologist. 2010;15 (supp 1): $5 \cdot 10$.

38. Nadler E, Eckert B, Neumann PJ. Do oncologists believe new cancer drugs offer good value? The Oncologist. 2006:11:90-5.

39. Hugues DA, Tilson L, Drummond M. Estimating drug cost in economic evaluations in Ireland and the UK. Pharmacoeconomics. 2009:27:635-43. 


\section{DISCUSIÓN}

GENERAL 
Discusión general

Las transformaciones sociales obligan a la adaptación de los modelos de asistencia sanitaria hacia nuevas formas de gestión, desde modelos gerenciales más rígidos hacia modelos organizativos basados en la gestión clínica.

A pesar de la ingente documentación recibida por los clínicos del hospital respecto al grado de eficacia y seguridad de nuevas tecnologías y moléculas, incluidos fármacos cada día de mayor complejidad, que se incorporan al arsenal farmacoterapéutico disponible, no se disponen de suficiente información acerca de su eficiencia, por lo que la aplicación de los estudios resultados sanitarios y específicamente los farmacoeconómicos, se convierten en una herramienta imprescindible para su análisis.

Sin embargo, su aplicación práctica en la rutina diaria no ha calado de manera generalizada en nuestros hospitales debido a diferentes razones, como la falta de tiempo, la presión asistencial, miedo o resistencia a la implantación de nuevas tecnologías, problemas éticos, existencia de otros criterios considerados más relevantes, poco conocimiento sobre los métodos de evaluación, el objetivo real es ahorrar y no lograr mayor eficiencia, problemas de fiabilidad de los estudios, los estudios no abordan los problemas diarios, entre otras.

Por todo ello es necesario acercar los conocimientos que aportan estos estudios para la toma de decisiones, y sobre todo, para que lleguen a los pacientes por su aplicación clínica diaria, rompiendo las distintas barreras que se deben superar para su definitiva implementación.

La farmacoeconomía tiene un alto valor como herramienta en la toma de decisiones a todos los niveles de las estructuras sanitarias. Ha sido demostrada la utilidad de los estudios farmacoeconómicos a nivel superior de la gestión de servicios sanitarios, es decir, de alta dirección o gestión de los servicios autonómicos de salud y/o gubernamental, es el nivel de la macrogestión. También se ha ido incorporando paulatinamente en otros niveles sanitarios, tanto públicos como privados. En efecto, como se detalla en el Capítulo 2 se utilizan para: a) Autorización para la comercialización de medicamentos, incluso en bastantes países 
por ley (Australia, Canadá, Portugal, Holanda, Finlandia, Noruega y Dinamarca, entre otros); b) Fijación de precios; c) Financiación pública de medicamentos (Reino Unido, Francia, Italia, Alemania, Suecia); d) Soporte de las decisiones sobre investigación y desarrollo en la industria farmacéutica; e) Definir estrategias de marketing de la industria farmacéutica; f) Utilización en la investigación clínica con medicamentos; g) Incorporación de medicamentos a formularios o guías farmacoterapéuticas; y finalmente, i) como soporte en las decisiones farmacoterapéuticas (Carrera-Hueso, 1998).

Estas dos últimas funcionalidades de este tipo de estudios, la g) y la i), se circunscriben más al nivel hospitalario de la meso y microgestión, siendo habitualmente utilizados sus resultados por las Comisiones de Farmacia Hospitalaria en su toma de decisiones, e incluso se han convertido los datos de eficiencia en la "cuarta barrera o garantía" a la hora de seleccionar un medicamento, tras los ya tradicionales: calidad, eficacia y seguridad (Del Llano J et al., 2008).

El servicio de farmacia, como eje vertebrador de la farmacoterapia que se aplica en el hospital, debe ejercer un liderazgo que necesariamente obliga a incorporar la farmacoeconomía como herramienta para introducir el criterio de eficiencia en todas sus decisiones sujetas a la gestión clínica.

La atención farmacéutica se define como la actuación responsable del farmacéuticos junto al resto del equipo multidisciplinar proveyendo un proceso farmacoterapéutico que mejore la calidad de vida del paciente considerado individualmente (Helper y Stand, 1990). No se ha demostrado su eficiencia, aunque disponemos ya de muchos datos que nos orientan a pensarlo, sin embargo contempla la corresponsabilidad del farmacéutico para obtener resultados sanitarios en el proceso farmacoterapéutico que provee el equipo multidisciplinar que atiende al paciente (Carrera-Hueso y Giráldez, 1995). Por ello, el farmacéutico debe liderar la traslación de los resultados de los estudios sanitarios a la práctica clínica diaria, lo que beneficiará a los pacientes y a la sociedad en su conjunto al utilizar los recursos limitados más eficientemente (Amat et al., 2011). 
Discusión general

Las principales estrategias para la incorporación de los estudios farmacoeconómicos en el hospital son: a) Utilizar la bibliografía publicada, b) Utilización de modelos farmacoeconómicos y c) realizar un ensayo clínico, pragmático o estudio observacional que recoja el componente de los costes de las alternativas (Carrera-Hueso, 1998; Sánchez, 1996). Se han detallado las mismas por su complejidad y coste de menor a mayor. Como es bien sabido el coste en tiempo y recursos de realizar un ensayo clínico o un estudio observacional es mucho mayor a extraer datos de la bibliografía. Actualmente se encuentra bastante acertada esta simple clasificación, aunque ya no es válido del todo extraer datos directamente de la bibliografía en mi opinión, a diferencia de otras aproximaciones más simplistas. A continuación haremos una descripción práctica de cada una de las estrategias de manera actualizada.

\section{a) Utilizar la bibliografía publicada.}

El grupo GENESIS de la SEFH ha desarrollado una metodología para la evaluación y selección de medicamentos que aplicada al medio hospitalario permite que las decisiones sean más independientes y eficientes. Este grupo incorpora una evaluación de todas las posibles alternativas utilizando el coste incremental respecto a la terapia de referencia para la adopción de las decisiones. Se utiliza principalmente para recomendar o no la incorporación de la nueva alternativa a la guía farmacoterapéutica o formulario del hospital (GENESIS, 2011, Puigventós et al., 2010).

En el Capítulo 9, Poquet y colaboradores nos muestran una revisión de la bibliografía sobre un grupo de medicamentos, los antineoplásicos orales, que en la actualidad su uso es creciente y cada día más importante, y no solamente desde el punto de vista de gasto presupuestario, sino que al racionalizar su utilización se logrará ahorrar grandes recursos hospitalarios y mejorar la cantidad y calidad de vida de los pacientes oncológicos.

Los autores concluyen que los nuevos citostáticos administrados por vía oral son menos tóxicos, reducen los costes indirectos, incluidos los costes de 
Discusión general

desplazamiento e implican una menor pérdida de tiempo para el paciente y sus cuidadores, pero sus costes deberían estar por debajo de un umbral (medido en coste/AVAC, actualmente se utiliza el de $30000 € /$ AVAC) que la sociedad aceptase gastarse en su adquisición, así como no dedicar esos recursos económicos a otras actividades.

A la hora de evaluar la información disponible en las publicaciones se debe utilizar un check-list, como el detallado en el Capítulo 2, o bien guías publicadas más recientemente, para asegurar la calidad de los resultados, exentos de sesgos, y que sirvan para reforzar las posibles decisiones a tomar (Carrera-Hueso, 1998; LópezBastida et al., 2010).

Esta estrategia también pueden utilizarse, no solo para decidir si incorporar un medicamento o no al formulario, sino para programar su inclusión, es decir cómo influirá la incorporación y a qué velocidad de las nuevas tecnologías (incluidos los fármacos) en nuestro quehacer diario (Camps et al., 2008; Delgado et al., 2009a).

Otros dos temas de decisión nada baladíes, que se pueden incluir inicialmente en este apartado, sin que suponga encorsetarlos estrictamente en esta clasificación, son:

1) Los estudios de impacto presupuestario: tan en boga hoy en día y que se encuentran en una etapa de desarrollo inicial pero abrumador y que sin duda se verán incrementados en un futuro próximo por su utilidad en el ámbito asistencial (Trueman et al., 2001; Brosa et al., 2005; Mar et al., 2008).

Por análisis de impacto presupuestario se entiende el estudio de la variación que sobre el presupuesto del financiador de una nueva intervención sanitaria provocará la introducción de la misma (Trueman et al., 2001).

Se debe utilizar para su elaboración la misma metodología que en un estudio coste-efectividad al uso, por lo que es indispensable conocerla. En efecto, debe explicitarse su perspectiva, horizonte temporal, el análisis de sensibilidad, la tasa de penetración o introducción de la nueva tecnología, etc. También se disponen de 
listas-guía para elaborar y evaluar un análisis de impacto presupuestario (Trueman et al., 2001; Brosa et al., 2005).

Los análisis de impacto presupuestario van a completar a los análisis costeefectividad o coste-utilidad a la hora de decidir como asignar de la mejor manera los limitados recursos sanitarios disponibles (Nuitjen y Rutten, 2000; Trueman et al., 2001).

Es evidente que un resultado positivo de una alternativa terapéutica no conduce de forma sistemática a una ampliación del presupuesto que puede destinarse a su financiación. El problema al que se enfrenta quien decide es que su presupuesto difícilmente puede modificarse a corto plazo. Por lo tanto, aunque pudiera mejorar la eficiencia del sistema en su conjunto, puede existir una incapacidad inicial para afrontar el incremento del gasto que supondría la introducción de esta nueva alternativa. El resultado de la evaluación económica está referido a todo el sistema, sin embargo, el decisor suele ser responsable solamente de una parte del presupuesto de dicho sistema, de "su presupuesto", lo que convierte a la factibilidad en el problema principal. El decisor sabe que no podrá contar con el ahorro que supone la incorporación de una nueva tecnología que se genere en otros ámbitos sanitarios.(Brosa et al., 2005)

Sin duda, los resultados de los análisis de impacto presupuestario son de especial interés tanto para gestores del hospital o área sanitaria así como para los jefes o directores de los SF, ya que les permite gestionar de manera más eficiente sus servicios o departamentos.

2) Adaptación o traslación de los resultados a nuestro ámbito de actuación. Es decir, utilizar datos propios e introducirlos o modificar los datos originarios, de tal manera que los modelos obtenidos difieren pero mejoran su extrapolación. Se utilizan mucho para adaptar estudios de una país a otro o de un sistema sanitario a otro. Por desgracia no se ha utilizado esta estrategia en el ámbito hospitalario como inicialmente podría esperarse, a pesar de que acercaría los resultados de muchos estudios a nuestra realidad clínica (ISPOR, 2003; López-Bastida et al., 2010). 


\section{b) Utilización de modelos farmacoeconómicos.}

El análisis de decisión es una técnica cuantitativa que es su aplicación al sector sanitario, pretende ayudar a resolver problemas, considerando todas las alternativas y todos los sucesos de interés, incluyendo las probabilidades de los acontecimientos. Los modelos de decisión se emplean con el fin de obtener información del mundo real (Ríos-Insua et al., 2002). En efecto, un modelo es una representación estructurada de los acontecimientos del mundo real o una aproximación sistemática a las condiciones reales, diseñado con el fin de evaluar el impacto de las estrategias médicas sobre los costes generados o los resultados obtenidos, bajo condiciones de incertidumbre. La modelización puede realizase mediante árboles de decisión, diagramas de influencia, modelos de Markov o mediante modelos de eventos discretos (Bootman et al., 2004; Gimeno et al., 2006; Díez-Vegas, 2005; Rodríguez-Barrios, 2004).

La utilización de modelos económicos en farmacoeconomía ha sido exponencial en los últimos años debido a las grandes ventajas que presenta frente a la realización de estudios diseñados expresamente, bien incluidos en ensayos clínicos o realizando estudios naturalísticos o pragmáticas (Soto, 2002; ISPOR, 2003; Rodríguez-Barrios, 2004). En efecto, la menor cantidad de recursos necesarios para llevar a cabo un modelo es su principal ventaja frente a las opciones anteriormente mencionadas, pero además tampoco están exentos de sesgos este tipo de diseños más pragmáticos, es decir, los estudios naturalísticos no son la panacea de los estudios farmacoeconómicos y sus principales limitaciones son (Rodríguez-Barrios, 2004).

- En los modelos no se dispone de resultados obtenidos en ensayos clínicos adecuados (se emplean datos procedentes de ensayos de escaso valor probatorio o, en el mejor de los casos, metanálisis de estos).

- En los modelos se pueden introducir datos sesgados procedentes de diferentes observaciones. 
- Se tropieza con dificultades para efectuar la extrapolación de datos clínicos.

- La posibilidad de manipular los modelos es mayor que la de manipular los resultados obtenidos en un ensayo clínico con distribución aleatoria. Los modelos pueden favorecer una determinada intervención basándose en hipótesis sesgadas, si no son lo suficientemente transparentes.

Los modelos farmacoeconómicos deben tener las siguientes características: ser explícitos y creíbles clínicamente (justificados); incorporar todas las intervenciones relevantes, ser transparentes y claros (sencillos y entendibles), los datos incorporados sean válidos, las asunciones sean debidamente explicadas, y argumentar la metodología utilizada (costes, tasa de descuento, etc.). A pesar de que la mejora en la metodología utilizada ha apuntalado con más firmeza estos modelos farmacoeconómicos, todavía quedan dudas sobre los mismos para trasladar sus resultados a la práctica clínica diaria. El Instituto Británico Nacional para la Excelencia Clínica (NICE) considera a los modelos farmacoeconómicos una importante herramienta metodológica para demostrar la eficiencia de las intervenciones evaluadas y por tanto asignar los recursos disponibles; considerando que éstos se utilizarán cada día más, sobre todo si se despejan las dudas de sus detractores, y por tanto es necesaria su estandarización para abandonar la etiqueta de "cajas negras" que les acompañan (Soto, 2002).

En los Capítulos 5 y 6 se realizan dos estudios sobre modelos de distintas situaciones en las que se intenta dar una respuesta objetiva a un problema clínico planteado. En ambos estudios se detallan la perspectiva hospitalaria, el horizonte temporal, las asunciones realizadas, las alternativas estudiadas y la estructura del modelo utilizado mediante un árbol de decisión, así como se extraen los costes y los beneficios, y la tasa de descuento que se aplica o no a los mismos (Carrera-Hueso et al., 2010; Carrera-Hueso et al., 2010a).

Los programas informáticos utilizados en el diseño, desarrollo y resolución de los modelos propuestos para el estudio coste-efectividad realizados en los Capítulos 
Discusión general

5,6 y 7 han sido: Microsoft Office Excel 2003, Data 3.5 (TreeAge Software 1998) y Elvira versión 0.162 (Data, 2011; Elvira, 2010). Se realizó el diagrama de influencia inicial del modelo con el programa Elvira, introduciendo todas las probabilidades de los acontecimientos y se resolvió mediante un árbol de decisión mediante el programa Data 3.5. El programa MS Excel ha servido de apoyo para comprobar todo el modelo.

En ambos estudios se utilizaron una cohorte de 10.000 pacientes por cada alternativa estudiada, obteniendo los suficientes casos para cada uno de los recorridos patológicos probables que pueden sufrir y se muestran en las ramas de cada uno modelo planteado.

Como marcan las guías metodológicas se describieron las asunciones de los modelos en ambos estudios coste-efectividad realizados, explicando el porqué de las mismas y de acuerdo con la evidencia publicada hasta ese momento (Soto, 2002; ISPOR 2003; Rodríguez-Barrios, 2004; López-Bastida et al., 2010).

Ambos estudios difieren metodológicamente en su horizonte temporal, uno cortoplacista como el del Capítulo 6, menor a un año el seguimiento que se realiza tras la utilización de dinoprostona en el parto, y otro a más largo plazo, el del Capítulo 5, en el que se realiza un seguimiento de la tromboprofilaxis tras cirugía mayor de cadera durante 5 años. Algunos autores proponen para los estudios de patologías crónicas utilizar modelos de Markov, ya que presentan más ventajas frente a los árboles de decisión (Rodríguez-Barrios, 2004; Dummond et al., 2005).

La tasa de descuento utilizada en el primer estudio del Capítulo 5 fue del $5 \%$ debido a que las condiciones de la coyuntura económica prevista en 2008, ya que todos los indicadores marcaban esta tendencia y estuvieron en torno a esta cifra durante el año 2007, tanto el euribor como el IPC; además suele ser la mas recomendada en todo tipo de países (Dummond et al., 2005). Como hemos comentado no se aplicó ningún descuento a los costes en el Capítulo 6 al realizar un seguimiento menor a un año. 
Discusión general

Los costes que se obtuvieron en ambos estudios fueron los sanitarios directos, en concordancia con la perspectiva del hospital tomada, de acuerdo con las guías y recomendaciones realizadas por expertos (Bootman et al., 2004; Dummond et al., 2005; López-Bastida et al., 2010).

Los costes promedio por paciente en el Capítulo 5 fueron de 1507 y $583 €$ para enoxaparina y fondaparinux, respectivamente. Sin embargo, el ratio coste efectividad para enoxaparina y para fondaparinux fue de 15,31 y de $1881,61 €$, por evento tromboembólico sintomático, respectivamente. Dicho de otra manera, el coste tromboembólico sintomático evitado fue 15,3 y 5,85€ para enoxaparina y fondaparinux, respectivamente. En el Capítulo 6 no se contemplaron costes indirectos ni intangibles, solamente se tuvieron en cuenta los costes directos sanitarios en cada alternativa, expresados en euros del año 2010: Los costes de todos los medicamentos utilizados se utilizó el Precio de Venta al Público y el resto de las cantidades monetarias han sido obtenidas del servicio de farmacia del hospital. Asimismo los costes por proceso, GRD ajustados, fueron facilitados por el servicio de contabilidad analítica de la Dirección de Gestión del Hospital Dr. José Molina Orosa, excepto para las hemorragias graves que se extrajeron de la base de datos Soikos, y el resto de datos fueron calculados a partir de las asunciones realizadas.

Los resultados de ambos estudios muestran una opción dominante respecto a la otra, al realizar un análisis coste-efectividad incremental de las dos opciones comparadas. En efecto, en el Capítulo 5 se deduce una dominancia absoluta del fondaparinux frente a la enoxaparina (RCEI=-782,93 € por evento evitado), al presentar una mayor efectividad y un menor coste total el pentasacárido. Asimismo en el Capitulo 6 la opción del Prepidilß 0,5 mg gel endocervical administrado dos veces al día es dominada por la opción de la administración de una dosis $10 \mathrm{mg}$ de Propess ${ }^{\circledR}$ vía vaginal para la maduración del cervix antes de la inducción del parto en mujeres gestantes nulíparas; ya que presenta el Prepidil $\circledast$ frente a Propess $₫$ un coste incremental de 669,6€ y una efectividad incremental de -1501 . 
Discusión general

Dado que es imposible eliminar la incertidumbre, el objetivo ha de ser su incorporación al estudio. El método más habitual para tratar el problema de la incertidumbre acerca de los parámetros es el análisis de sensibilidad (ISPOR, 2003; Drummond et al., 2005; Rubio-Terrés et al., 2004; López-Bastida et al., 2010). La incertidumbre sobre los costes, los beneficios y las relaciones entre ambos puede tener un doble origen. Por un lado, puede existir incertidumbre acerca de los parámetros. Esto ocurre cuando no puede conocerse con certeza cuáles son los verdaderos valores numéricos de tales parámetros. Por otra parte, podemos enfrentarnos a la incertidumbre sobre el modelo, esto es, incertidumbre acerca de cuál es la forma óptima de combinar estos parámetros (ISPOR, 2003).

En los capítulos 5 y 6 se realizó un análisis de sensibilidad univariado como multivariante con el fin de contrastar la robustez de los resultados. En el estudio del capítulo 5 uno de los parámetros variado fue la tasa de descuento, entre el $3 \%$ y el $7 \%$, y además se variaron 5 parámetros más, explicando tanto esta selección como sus valores de acuerdo con la evidencia disponible. En el otro estudio se modificaron los valores de los parámetros simulando 9 escenarios posibles. En ambos estudios, estos análisis de sensibilidad refuerzan los resultados obtenidos al obtener la misma decisión que en los casos base analizados.

En efecto, la forma tradicional de análisis de sensibilidad es el análisis univariado. El modo de proceder consiste en modificar el valor de un único parámetro y observar cómo cambian los resultados. A continuación el procedimiento se repite con cada uno de los parámetros sujetos a incertidumbre -uno sólo cada vez-, manteniendo el resto de parámetros constantes. El análisis de sensibilidad univariado permite identificar los parámetros que resultan más influyentes en los resultados finales y sirve para valorar el grado de robustez del análisis. Sin embargo, y pese a ser el más usado, este tipo de análisis no es teóricamente el más correcto. La razón principal estriba en que en la realidad los parámetros no son independientes, o incluso aunque lo sean, no suelen variar de forma aislada. Otra razón por la cual el análisis univariado no es la mejor opción desde un punto de vista teórico radica en el hecho de que lo que interesa en una evaluación económica es la 
relación entre los costes y los beneficios, normalmente expresada mediante un cociente -la ratio coste-efectividad-, y la variabilidad de dicho cociente es mayor que la variabilidad independiente del numerador y el denominador por separado (Puig-Junoy y Pinto JL, 2008; Willan y Briggs, 2006).

La incertidumbre sobre el valor verdadero de los parámetros puede tener varias causas (Gimeno et al., 2006): a) en primer lugar determinados parámetros no pueden ser observados; un ejemplo es la efectividad a largo plazo en algunos tratamientos, cuyo conocimiento exigiría que la duración del estudio fuese muy larga y raramente se sigue a los pacientes de forma prolongada en el tiempo; b) una segunda causa de incertidumbre se deriva de la ausencia de consenso teórico acerca del valor más apropiado para un parámetro; por ejemplo, hay opiniones muy diversas sobre la forma de estimar el coste de oportunidad del tiempo perdido por pacientes o familiares que no están trabajando; c) en tercer lugar, puede existir incertidumbre en relación con aspectos básicos del tratamiento; por ejemplo, puede que la epidemiología de la enfermedad, la conducta del médico o la adherencia al tratamiento sean poco conocidas; y d) otra fuente de incertidumbre son las posibles divergencias entre la población de la cual se han obtenido los valores de los parámetros y la población en la cual se quiere estimar el coste-efectividad (Soto, 2002; Darbá, 2006).

Como se puede ver, la incertidumbre que afecta a los parámetros puede deberse a muy diversas causas y ser originada por diferentes fuentes. Están sujetos a incertidumbre los datos procedentes de ensayos clínicos, los estimados mediante el meta-análisis de la literatura, los sugeridos por expertos, los estimados mediante modelización, etcétera. Prácticamente cualquier parámetro incluido en el análisis es susceptible de provocar incertidumbre sobre el resultado de una evaluación económica (Drummond et al., 2005).

El análisis de sensibilidad tradicional es un método determinístico -por oposición a los métodos probabilísticos- que trata de aislar los efectos de la variación de los valores de los distintos parámetros para que el analista pueda 
observar la influencia que dicha variación tiene sobre el resultado final del estudio (Rodríguez-Barrios, 2004).

Existen varias alternativas al análisis univariado a la hora de tratar la incertidumbre. En efecto, disponemos del análisis multivariado, el "análisis de escenarios extremos", el "análisis de umbral", y por último el análisis de sensibilidad probabilístico (Drummond et al., 2005; Bootman et al., 2004). Muchos autores recomiendan que se utilice el análisis de sensibilidad probabilístico, con la simulación de Monte-Carlo, para testar los parámetros de manera conjunta y minimizar los problemas del análisis univariado (Darbá, 2006; López-Bastida et al., 2010).

Las limitaciones del análisis de sensibilidad determinístico se solucionan mediante el probabilístico, donde se propaga la incertidumbre presente en cada uno de los parámetros del modelo mediante distribuciones probabilísticas (gamma, beta, log-normal, etc.) y simulaciones de Monte Carlo (normalmente se simulan 1.000 o 10.000 resultados, dependiendo de la dificultad del modelo), y creando distribuciones conjuntas de costes y efectos que representan simultáneamente la incertidumbre presente en cada uno de los parámetros. Las distribuciones utilizadas no son para nada arbitrarias y dependen de los datos observacionales obtenidos, del tipo de parámetro modelizado y del proceso de estimación utilizado. Por tanto no todos los pacientes se comportan como el paciente medio y el modelo refleja explícitamente la variabilidad que puede haber entre los distintos pacientes dentro de una cohorte (Darbá, 2006). La interpretación del método probabilístico normalmente se realiza por medio de la nube de puntos en el plano coste-efectividad y en las curvas coste-efectividad (Willan y Briggs, 2006).

Por tanto, una de las limitaciones que presentan los estudios descritos en los Capítulos 5 y 6 , es que no se ha realizado un análisis de sensibilidad probabilístico. Sin embargo, cuando éste se realiza no implica que cambie obligatoriamente la decisión última a tomar por el decisor sino más bien al contrario, suele confirmarse. También es de mucha utilidad realizar el análisis de sensibilidad estocástico para identificar subgrupos de pacientes, con características especiales, que se 
Discusión general

beneficiarían más de la tecnología evaluada (Claxton et al., 2005; Puig-Junoy y Pinto $\mathrm{JL}, 2008)$.

En el estudio del Capítulo 5, el menor coste por paciente del grupo de fondaparinux se obtuvo por un menor coste en el tratamiento de los episodios tromboembólicos, tanto los inmediatos como los tardíos (síndrome postrombótico, tromboembolismo pulmonar tardío o trombosis venosa profunda recurrente). EI mayor número de hemorragias mayores y del coste de adquisición del fármaco no compensa este ahorro obtenido de $924 €$ respecto a la enoxaparina, medicamento de referencia en nuestro medio. En otro estudio obtuvieron los mismos resultados pero la diferencia entre ambos tratamientos profilácticos fue menor, de 52,5€, aunque la distribución de costes proporcionalmente fue muy similar a los obtenidos en nuestro trabajo para ambos grupos de pacientes tratados (Lundkvist et al., 2007). Estas discrepancias en costes con el estudio sueco, pueden ser debidas a que en este trabajo se utilizó un modelo simplificado y el coste de referencia fue diferente tanto para el tratamiento de un episodio de trombosis venosa profunda en hospitales como para tratar las hemorragias mayores. En el estudio comparable al nuestro (Lundkvist et al., 2007) se tomaron valores de 1095,1 $€$ y 1483,3 $€$ (euros año 2006) respectivamente, mientras que en nuestro trabajo se ha tomado un coste de $4172,71 €$ y de $3075,27 €$ (euros año 2008), similar al seleccionado por otros autores (Wade et al., 2004; Slof y Badia, 2006). Las diferencias también pueden ser atribuidas a la distinta situación geográfica o a la toma de diferentes años del valor de la moneda. En nuestro caso es poco plausible que sean debidas a estas razones ya que sería como asegurar que la asistencia sanitaria en Suecia es mucho más barata que en España. Sin embargo, en ambos trabajos las probabilidades de los eventos han sido muy parecidas al tomar como referencia el estudio PENTHIFRA Plus (Eriksson et al., 2003). Otra posible explicación de esta amplia diferencia es que el tamaño del efecto de la tromboprofilaxis es mayor en los pacientes intervenidos de fractura de cadera que en los sometidos al resto de cirugía ortopédica mayor, artroplastia total de rodilla o cadera (Bauer et al., 2001; Lassen et al., 2002; Turpie et al., 2002). 
Discusión general

No se encontraron datos publicados de los sucesos ocurridos en tromboprofilaxis extendida con fondaparinux tras intervención de cirugía ortopédica mayor: prótesis de cadera, prótesis de rodilla y fractura de cadera. Tan sólo se encontraron datos en un horizonte temporal alto de 5 años para los pacientes intervenidos de fractura de cadera con fondaparinux (Eriksson et al., 2003), por lo que han sido utilizados también para enoxaparina.

Otra limitación del análisis del Capítulo 5 es que no consideraron reducciones en calidad de vida que los pacientes sufren cuando tienen un evento tromboembólico venoso, que sí ha sido tenido en cuenta en otros estudios similares (Haentjens et al., 2004). Sin embargo la no inclusión de las posibles pérdidas de calidad de vida en el modelo es una actitud conservadora, ya que favorecería en el análisis coste-efectividad a la alternativa dominada (enoxaparina), porque con fondaparinux la probabilidad de sufrir estos sucesos es menor.

En el modelo de decisión del Capítulo 6 no se tuvo en cuenta la mortalidad de las gestantes ni de los recién nacidos; afortunadamente su prevalencia es muy baja. Su registro diferenciado, es decir de mujeres nulíparas con el cervix inmaduro que serian la población diana de nuestro estudio, del total de mortalidad infantil y de la mortalidad de las gestantes no ha sido posible obtenerla, seguramente porque se necesiten series de pacientes muy grandes para poder registrarlas debido a su muy baja incidencia (SEGO, 2004).

Existen alternativas farmacológicas a la dinoprostona, recientemente se ha comercializado el misoprostol (análogo sintético de la Prostaglandina $E_{1}$ con resultados prometedores) como comprimidos vaginales (Misofar ${ }^{\circledR}$, a dosis de 25 y $200 \mathrm{mcg}$ ). Existen suficientes evidencias para su utilización exitosa en la maduración del cervix e incluso hay estudios que lo recomiendan por tener mejor relación coste efectividad que la Prostaglandina $\mathrm{E}_{2}$ (Wing et al., 1995).

Gracias a los resultados publicados por sobre las preferencias reveladas por mujeres gestantes australianas mediante su disponibilidad a pagar sobre todos los aspectos relacionados con el tratamiento con gel endocervical de dinoprostona o con oxitocina y ruptura artificial de membranas, se abre la posibilidad de realizar estudios coste-utilidad en esta indicación (Taylor y Armour, 2003). El modelo de decisión presentado en este trabajo puede perfectamente utilizarse para realizar 
estudios de coste-utilidad ya que en la práctica asistencial cada día se tienen más en cuenta las opiniones de los pacientes acerca de su tratamiento.

La incertidumbre puede afectar, no ya a los valores de los parámetros utilizados en el análisis como hemos visto, sino a la forma funcional del modelo que combina tales parámetros. Cuando esto ocurre, es decir, cuando no se tiene certeza sobre la forma matemática en que se combinan determinados parámetros, no existen métodos claros para proceder a su tratamiento e incorporación al análisis. En cualquier caso, lo que sí se ha de hacer es reconocer en el informe esta incertidumbre así como explicitar la opción metodológica elegida (Soto, 2002; ISPOR, 2003).

En realidad, una de las pocas sugerencias que se puede hacer al respecto consiste en llevar a cabo un análisis de sensibilidad de los modelos. Es decir, se podrían calcular las ratios coste-efectividad bajo los dos modelos alternativos y comprobar la magnitud del cambio (ISPOR, 2003).

En el campo médico no se realiza habitualmente un análisis de sensibilidad estructural de los modelos utilizados debido a múltiples factores, sobre todo a su alta complejidad y coste de recursos. En una revisión de las guías publicadas solamente 4 de las 16 seleccionadas como excelentes recomendaban un análisis estructural del modelo, aunque la mayoría de ellas indicaban que se deben justificar las asunciones consideradas en su elaboración y además la relación entre las variables incluidas en el modelo (ISPOR, 2003, Akehurst et al., 2000).

En el Capítulo 7 (Carrera-Hueso y Ramón, 2011) realizamos un análisis de sensibilidad estructural del modelo propuesto en el estudio descrito en el Capítulo 6 (Carrera-Hueso et al., 2010).

El resultado es que no varía la decisión seleccionada en el modelo uno y dos respecto al base con este tipo de análisis de sensibilidad. En efecto, al eliminar como variable independiente las hemorragias post-parto del modelo base e incluirla en la de efectos adversos se obtiene el modelo 1 del análisis de sensibilidad estructural obteniéndose una ratio coste-efectividad incremental fue de $-0,20 €$, lo 
que supone un ahorro de 20 céntimos por cada parto sin complicaciones al utilizar Propess $₫$ en vez de Prepidil $\circledast$ gel. Por otra parte, al considerar todos los efectos adversos de los medicamentos en el mismo nodo que representa esa variable, es decir incluyendo la hiperestimulación uterina y la hemorragia post-parto, obtenemos el modelo 2 dándonos el mismo resultado, es decir la decisión óptima en mujeres gestantes nulíparas y que presenten el cuello uterino inmaduro sigue siendo la utilización de una dosis de Propess ${ }^{\circledR} 10 \mathrm{mg}$ vía vaginal para la maduración del cervix antes de la posible inducción o no del parto. El ratio coste-efectividad incremental obtenido fue de $-0,45$, similar al modelo base, lo que nos da una idea de la similitud del modelo utilizado con el modelo de referencia, al cambiar su estructura.

No todas las guías incorporan la realización del análisis de sensibilidad estructural, aunque sí describen la metodología para que se minimicen los sesgos en la estructura del modelo seleccionado antes de realizar el estudio. Para minimizar los errores se suelen utilizar checklist que sirven de guía a los propios investigadores o de control a los evaluadores o decisores a los que va dirigido el informe final (ISPOR, 2003). Sobre este tipo de recomendaciones, la mayoría establece que debe de simplificarse al máximo el modelo, reflejar escrupulosamente la realidad del problema clínico que nos ocupa, establecer claramente el objetivo y la hipótesis del modelo, describir el modelo y su estructura detalladamente, decidir para qué horizonte temporal y perspectiva se utilizará, describir las alternativas que se evalúan, tener en consideración todas las variables importantes que afecten a los resultados tanto económicos como clínicos, establecer las fuentes de información tanto de efectividad como de efectos secundarios y costes; y por último justificar cada una de las elecciones realizadas así como las asunciones contempladas en nuestro modelo basadas en la mejor evidencia científica disponible. Por tanto, no es posible incorporar al modelo la situación más improbable o "caso raro" que se nos presente, ya que por una parte aumentaríamos su complejidad y por otra su contribución sería prácticamente despreciable para resolver la incertidumbre planteada (Soto, 2002). 
En medicina no se realizan normalmente cambios en la estructura del modelo como ocurre en la ingeniería, arquitectura, finanzas o economía (Ríos-Insua et al., 2002). Cierto es que los resultados obtenidos en intervenciones sucesivas nos puede conllevar resultados muy diferentes e incluso aberrantes, circunstancia que se da en raras ocasiones en otros campos (Díez-Vegas, 2005). Por ejemplo, no es posible anteponer la variable muerte a ninguna otra ya que es un resultado final en sí misma o poner la administración de un medicamento tras sus efectos secundarios; sin embargo, es posible en la construcción de un puente poner en el árbol de decisión la construcción de la plataforma antes que la de pilotes, o viceversa, de tal manera que se extraiga la situación más eficiente.

En efecto, se ha realizado un trabajo con el objetivo de extraer un documento de consenso o guía a partir de 16 listas-guía o checklist publicados considerados excelentes, para que pueda ser aplicado en la toma de decisiones dentro del propio NICE y cuyo resultado puede ser consultado, determinando la manera y pasos a seguir a la hora de realizar un modelo garantizando su fiabilidad, validez interna y externa (ISPOR, 2003; López-Bastida et al., 2010).

La estructura del modelo es claramente una fuente de desconfianza para trasladar sus resultados a la práctica clínica diaria, siendo el análisis de sensibilidad estructural una herramienta útil para despejar estas dudas, reforzando los resultados obtenidos en las evaluaciones económicas por mejorar su fiabilidad, al igual que sucede en el análisis de sensibilidad de los parámetros y el análisis de sensibilidad probabilístico (Claxton et al., 2005).

Al comparar cuantitativamente el modelo 2 con el modelo base, al considerar exactamente los mimos valores o resultados a priori pero en variables diferentes, se obtienen resultados del análisis coste-efectividad similares. Al eliminar una variable cuya incidencia es muy baja como las hemorragias post-parto debida al tratamiento, se comprueba que aunque no varía la decisión final en la toma de decisiones, la variación de los resultados obtenidos en el modelo 1 es mayor respecto al modelo base. Con ello deducimos que se debe tener en cuenta la variable hemorragias postparto en el modelo, independientemente si se incorpora de manera independiente o 
incorporada en la variable de efectos adversos. Posiblemente se corrobore con este ejemplo que el análisis de sensibilidad realizado estructural en modelos sencillos no afecte a los resultados, quedando sin clarificar si se debe aplicar de manera sistemática.

En nuestro trabajo del Capítulo 7 hemos utilizado los diagramas de influencia para obtener los distintos modelos clínicos, por su mayor flexibilidad e intuición para representar las variables implicadas. Son considerados un tipo de modelización gráfica más avanzada al obtener con ellos relaciones causales (Stahl, 2008).

Hasta hace relativamente poco tiempo los árboles se presentaban como la única forma de representar y evaluar un problema de decisión dinámico, con varias decisiones encadenadas a tomar. Los diagramas de influencia fueron concebidos inicialmente como método de representación más compacta de problemas, y posteriormente se resolvían como árboles de decisión. En los años 80 se introdujeron algoritmos de evaluación de diagramas de influencia que funcionaban directamente en el propio diagrama, convirtiéndose en un lenguaje grafico de modelización (Ríos-Insua et al., 2002).

Un diagrama de influencia es un grafo dirigido acíclico con tres tipos de nodos: de azar o aleatorios, de decisión y un nodo de valor o utilidad; y con dos tipos de arcos dirigidos: arcos condicionales que apuntan a nodos de azar o al de valor, y arcos informativos, que lo hacen a los nodos de decisión. (Ríos-Insua et al., 2002; Díez-Vegas, 2005).

Recordemos que un grafo viene dado por un conjunto de nodos y un conjunto de enlaces entre los nodos. Cada enlace se define formalmente como un par de nodos. En este curso vamos a estudiar solamente grafos en los que cada enlace une dos nodos diferentes. También vamos a exigir que entre cada par de nodos haya como máximo un enlace.

Cada nodo aleatorio (más propiamente, para cada nodo que representa una variable aleatoria) Ileva asociada una tabla de probabilidad condicional dados sus 
padres; observe que los padres de un nodo aleatorio pueden ser tanto nodos aleatorios como nodos de decisión. El nodo utilidad lleva una tabla que indica la utilidad en función de los valores de los padres de este nodo (Díez-Vegas, 2005).

\begin{tabular}{|l|l|l|}
\hline$\downarrow$ Destino / Origen $\rightarrow$ & Variable aleatoria $X$ & Decisión $D$ 1 \\
\hline Variable aleatoria $Y$ & $\begin{array}{l}X \text { influye causalmente de } \\
\text { forma directa sobre } Y\end{array}$ & $\begin{array}{l}\text { La decisión } D \text { 1 influye } \\
\text { directamente sobre } Y\end{array}$ \\
\hline Decisión $D 2$ & $\begin{array}{l}\text { Al decidir } D 2 \text { se conoce } \\
\text { ya el valor que ha tomado } \\
X\end{array}$ & $\begin{array}{l}\text { La decisión } D \text { 1 se toma } \\
\text { antes que } D 2\end{array}$ \\
\hline Utilidad $U$ & $\begin{array}{l}\text { El valor que toma } X \\
\text { influye directamente en la } \\
\text { utilidad }\end{array}$ & $\begin{array}{l}\text { La decisión } D \text { 1 influye } \\
\text { directamente en la utilidad }\end{array}$ \\
\hline
\end{tabular}

Una de las formas de evaluar un diagrama de influencia consiste en desarrollar y evaluar el árbol de decisión asociado. Para ello, lo primero que hay que hacer es determinar el orden en que aparecen las variables en el árbol, y para ello hay que respetar rigurosamente las cuatro reglas siguientes (Díez-Vegas, 2005):

- Regla 1: a la derecha del todo debe aparecer el valor de la utilidad para cada rama.

- Regla 2: si la decisión D1 se toma antes que la decisión D2, el nodo D1 debe aparecer a la izquierda del nodo D2.

- Regla 3. Las variables cuyo valor se conoce antes de tomar la decisión $\mathrm{D}$, han de aparecer a la izquierda del nodo $\mathrm{D}$ en el desarrollo del árbol; las que no se conocen al decidir $\mathrm{D}$, aparecerán a la derecha.

- Regla 4. De las dos reglas anteriores se deduce que, si el valor de la variable $\mathrm{X}$ se conoce después de la decisión $\mathrm{D} 1$ y antes de la $\mathrm{D} 2$, el nodo $X$ debe aparecer entre D1 y D2. 
Es importante señalar que la ausencia de un enlace entre una variable aleatoria $\mathrm{X} \rightarrow \mathrm{D}$ indica que en el momento de decidir si se aplica el tratamiento, no existe evidencia directa de si la persona padece la enfermedad $\mathrm{X}$, sino sólo evidencia indirecta a través de otra variable interpuesta aleatoria $Y$ (Ríos-Insua et al., 2002; Díez-Vegas, 2005).

También es importante la ausencia de un enlace entre una variable aleatoria $\mathrm{Y} \rightarrow \mathrm{U}$, pues indica que el resultado de la prueba no influye directamente en el estado de salud del paciente; sí influye indirectamente por dos motivos (Díez-Vegas, 2005):

- porque aporta evidencia sobre $\mathrm{X}$, de modo que una persona que da positivo en $Y$ tiene más probabilidad de padecer la enfermedad $y$, por tanto, peor pronóstico (esta influencia se transmite por el camino $\mathrm{Y}-\mathrm{X}-\mathrm{U}$ );

- porque, como veremos al evaluar el árbol, la decisión que tomemos dependerá del resultado de la prueba (influencia por el camino $Y-D-U$ ), por lo que un verdadero positivo o un verdadero negativo ayudan a tomar la decisión correcta, mientras que un falso positivo o un falso negativo llevan a tomar una decisión perjudicial.

La modificación de la estructura del modelo nos conduce a determinar qué modelo es más sencillo, transparente y fiable; y por tanto más entendible por todos los implicados en el proceso de toma de decisiones. Lo que conduce a seleccionar el mejor modelo posible en una situación clínica determinada y explicar el porqué de la misma. Esta selección puede incluso ahorrándonos recursos, eliminando variables o costes del modelo al no influir en los resultados finales estudiados o en la eficiencia comparada de las intervenciones, por ejemplo el análisis coste-utilidad o coste efectividad incremental.

Las conclusiones que podemos extraer o inferir de la realización de un análisis de sensibilidad estructural en los estudios farmacoeconómicos son:

- Es posible realizar en el campo sanitario un análisis de sensibilidad estructural congruente con la situación clínica estudiada. 
- Cada uno de los modelos propuestos para realizar el análisis de sensibilidad estructural debe ser validado clínicamente.

- La utilización de diagramas de influencia permite llevar a cabo el análisis de sensibilidad de manera más intuitiva y sin grandes esfuerzos, respecto a los árboles de decisión.

- Aunque la reducción de la incertidumbre obtenida con el análisis de sensibilidad estructural puede no ser muy alta, sin duda aporta información valiosa sobre el problema que se quiere representar y da una mayor validez y fiabilidad al modelo.

Más recientemente otros tipos de modelos se están empezando a utilizar en el ámbito sanitario: son los modelos de simulación de eventos discretos. Sirven de alternativa a los modelos tradicionales (árboles de decisión y modelos de Markov) permitiendo salvar las limitaciones de los anteriores. Estos modelos ya fueron utilizados ampliamente en otros campos del conocimiento. (Stahl, 2008).

Las principales limitaciones de los modelos de Markov, que se evitan con este tipo de modelos, son principalmente que no tienen memoria de ciclos anteriores, es decir que se "olvida" de la historia clínica del paciente, al tener siempre la misma probabilidad de transferir a otro estado de salud ya que los periodos de tiempo o ciclos son prefijados y constantes; además puede obligar al analista a realizar asunciones del modelo que sean poco realistas con la clínica (Rodríguez-Barrios et al., 2008; Stahl, 2008).

Los modelos de simulación de eventos discretos permiten modelar situaciones tan complejas, que en muchos casos, no hay soluciones analíticas, y es necesaria su resolución mediante técnicas de simulación. Por tanto, permiten representar la enfermedad y su manejo de una manera más flexible, con menos restricciones y por tanto, más asimilable a la realidad que los modelos de Markov. Los modelos de eventos discretos se utilizan para estudiar sistemas y procesos cuyo estado va cambiando. Todos los aspectos relevantes de la enfermedad pueden incorporarse 
de manera explícita y eficiente. El modelo, en su conjunto, puede presentarse de forma transparente y sencilla, y tienen una aplicación importante como instrumento de la actividad clínico asistencial y en el análisis de necesidades de gestión en listas de espera (Rodríguez-Barrios et al., 2008; Stahl, 2008).

Gráficamente los modelos de eventos discretos se pueden considerar redes complejas. Cada interacción entre entidades, que son los elementos dinámicos y normalmente los pacientes, es un evento ya que cambia el propio sistema en su totalidad además del estado de la entidad envuelta. El tiempo entre eventos puede manejarse probabilísticamente, usando incrementos de tiempo fijos o ambos, según se parezca más a la realidad (Stahl, 2008). Por tanto los eventos es todo aquello que puede ocurrir durante la simulación en función del proceso que estamos estudiando, como por ejemplo un efecto adverso o un ingreso hospitalario (Monleón y Ocaña 2006).

Otros elementos del modelo son (Rodríguez-Barrios et al., 2008):

- las variables que definen el modelo: el horizonte temporal, la tasa de descuento o la perspectiva;

- los recursos: personas, bienes, espacios y otros;

- los acumuladores estadísticos: son variables que acumulan información de los que ha pasado sin participar en el suceso, son pasivos, ejemplo cociente coste-efectividad;

- los atributos: son las características de las entidades como la edad, sexo, etc.,

- las colas; cuando un paciente utiliza un recurso deja de estar disponible para otros,

- los retrasos: que pueden ser explícitos (tiempo de visita médica) o implícitos (lista de espera para un TAC), 
- ecuaciones de riesgo y generadores de números aleatorios: pueden ser funciones o distribuciones estadísticas de probabilidad (beta, gamma, exponencial, etc.), y

- reloj de simulación: avanza hasta el siguiente evento y actualiza las variables, repitiéndose de forma indefinida.

Su gran desventaja es que requieren de mayor cantidad de datos y pueden necesitar un mayor tiempo de ejecución, así como el manejo de programas informáticos complejos para su diseño (Monleón y Ocaña 2006; Rodríguez-Barrios et al., 2008).

Las principales etapas para realizar un estudio de eventos discretos son (Monleón y Ocaña 2006; Rodríguez-Barrios et al., 2008): a) formulación de la problemática y determinación de objetivos, b) modelado del sistema, c) implementación del modelo en el ordenador, d) verificación del programa, e) validación del modelo, f) diseño de la simulación y prueba piloto, g) ejecución de la simulación, h) análisis de resultados y, i) documentación del experimento.

\section{c) Realización de un ensayo clínico, pragmático o un estudio observacional.}

A nadie se nos escapa que la realización de un estudio para la resolución de un problema clínico es más costoso, en recursos personales y económicos, y nos conlleva mayor tiempo que la realización de un modelo matemático. Sin embargo en ocasiones esto no será posible como explicamos en el Capítulo 2 (Carrera-Hueso, 1998).

Aunque no hemos diseñado un estudio naturalístico o pragmático para contestar una pregunta de investigación clínica, hemos realizado en el Capítulo 4 un seguimiento de un paciente epiléptico y vemos cómo influye el tratamiento farmacoterapéutico en su calidad de vida, CVRS, en varias medidas repetidas a lo largo del tiempo por el SF-36 (Ware y Sherbourne, 1992; Carrera-Hueso, 1997b). 
En este estudio aunque hayamos utilizado un instrumento genérico únicamente, a diferencia de la recomendación de utilizar conjuntamente con uno específico también (Bungay et al., 2005; Fayers y Machin, 2007), comprobamos la correspondencia de la CVRS del paciente con la clínica.

Hay que subrayar que en aquella época los estudios de CVRS estaban iniciándose y los cuestionarios se utilizaban únicamente para diferenciar entre poblaciones, es decir, la población en general y otra población de pacientes o con ciertas discapacidades. No existía en aquella época un cuerpo de disciplina para la interpretación clínica de las medidas de CVRS como existe actualmente con la aplicación de la teoría de la respuesta a los ítems. Actualmente se utilizan para valorar el efecto de un tratamiento a partir de diferencias grupales (Viladrich y Doval, 2011): a) el tamaño del efecto, b) el índice de Guyatt, c) la respuesta media estandarizada y d) el índice de cambio fiable.

La interpretación de las medidas de sensibilidad al cambio, como otros tantos índices psicométricos, está plagada de reservas. En primer lugar se trata de medidas dependientes de la población a las que se aplican; en segundo lugar por la posible atenuación por falta de fiabilidad de las medidas, y finalmente por no tener en cuenta el efecto de regresión a la media que se produce en los datos longitudinales (Viladrich y Doval, 2011).

Actualmente se han incorporado todo tipo de instrumentos de calidad de vida, fiables y validados, requisitos que se revisan en el Capítulo 3 (Carrera-Hueso, 1997), a la práctica clínica diaria (Fayers y Machin, 2007; Walters, 2009). La CVRS es un resultado en sí mismo e incorpora la opinión de los pacientes al proceso farmacoterapéutico por lo que el profesional sanitario debe conocer, manejar e interpretar este tipo de datos que nos ofrecen los cuestionarios.

También son útiles los estudios de resultados sanitarios para la implantación de nuevos servicios o programas asistenciales en el hospital o en su ámbito de actuación. Podemos nombrar varias experiencias realizadas en nuestra Comunidad Autónoma que centran su análisis en el ámbito hospitalario y el ambulatorio, por 
ejemplo una relativa a la implantación de un sistema novedoso de citación de consultas en oncología en Valencia (Camps et al., 2011); y otra, el programa Consúltenos, que se refiere al consejo farmacéutico para el uso correcto de medicamentos al alta hospitalaria de los pacientes (Pardo et al., 2008).

Desde hace más de veinte años, se han establecido servicios de farmacia que han permitido la intervención clínica del farmacéutico de hospital, de manera recíproca a la incesante innovación a la farmacoterapia. Sin embargo, se ha demostrado que muchos de los ingresos hospitalarios (Johnson y Bootman, 1995), consultas de urgencias y problemas de salud durante el ingreso guardan relación con la medicación utilizada por los pacientes (Calleja et al., 2008). Se han diseñado estrategias, fundamentalmente el seguimiento farmacoterapéutico incluido en programas de atención farmacéutica (Helper y Strand, 1990), para el abordaje de esta problemática aunque con dispar resultado, tanto en nuestro país como en el extranjero. Además existen diferentes criterios de intervención farmacéutica que dificultan analizar y comparar los resultados obtenidos con los programas de atención farmacéutica (Silva-Castro et al., 2010).

Por lo anteriormente descrito, existen dudas reales acerca de los beneficios en salud que producen los programas de atención farmacéutica y condiciona su aplicación o generalización al resto del Sistema Sanitario. En efecto, se intuye que su aplicación puede redundar un beneficio en los pacientes atendidos pero sin tener claro a que coste, por lo que su eficiencia no está ni mucho menos aclarada (Peiró, 2006).

Por mencionar un ejemplo que corrobora lo expuesto hasta el momento son los estudios que demuestran un ahorro de costes por la atención farmacéutica en las unidades de cuidados intensivos, entre aproximadamente 206.000\$-280.000\$ (Kopp et al., 2007). Aunque la mayoría de los estudios publicados presentaron un número de carencias metodológicas importantes como ausencia de grupo control, limitada perspectiva en la recogida de costes y resultados, enfoque en costes sanitarios directos, exclusión de potenciales beneficios en salud, exclusión de los costes 
laborales de los farmacéuticos y ausencia de un análisis de costes incremental (De Rijdt et al., 2008).

Los SF necesitan tomar decisiones acerca de qué programas deben implantar en sus hospitales para mejorar su atención, y los directores o jefes de los mismos carecen en muchos casos de datos fiables para justificarlos, especialmente ratios coste-beneficio para desarrollar programas clínicos de atención farmacéutica (Anderson y Schumock, 2009).

Por lo que para justificar los servicios clínicos de los SF es necesario no mostrar únicamente el posible ahorro sino que se obtienen resultados en salud que beneficien a los pacientes atendidos y a la sociedad en general. En resumen, la implantación de servicios o programas se verán reforzados si se obtienen resultados sanitarios positivos con los mismos, tanto clínicos como humanísticos y de costes, otorgándoles mayor valor por demostrar su eficiencia. 
CONCLUSIONES 
Del presente trabajo se puede concluir:

1. Es factible aplicar los estudios de resultados sanitarios en la práctica hospitalaria diaria, tanto a nivel de Comisiones Clínicas como para la atención de los pacientes.

2. La traslación de los resultados sanitarios de los estudios, previa adaptación a nuestro medio, permiten tomar mejores decisiones al disponer de mayor información fiable.

3. La realización de modelos farmacoeconómicos tanto para reflejar nuestra realidad clínica como para corroborar otras en diferentes países por ejemplo, permiten incorporar todos los posibles resultados sanitarios en la toma de decisiones clínicas.

4. Los modelos farmacoeconómicos no deben restringirse a los árboles de decisión, sino utilizar todas las opciones que nos ofrecen otras tecnologías como los modelos de Markov, los diagramas de influencia o los modelos de eventos discretos.

5. El análisis de sensibilidad, ya sea determinístico o estocástico, es una herramienta necesaria para verificar la robustez de los resultados obtenidos con los estudios realizados.

6. Es posible realizar en el campo sanitario un análisis de sensibilidad estructural congruente con la situación clínica estudiada y aunque la reducción de la incertidumbre obtenida con el mismo puede no ser muy alta, sin duda aporta información valiosa sobre el problema que se quiere representar y da una mayor validez y fiabilidad al modelo.

7. La calidad de vida relacionada con la salud es un resultado en sí mismo por lo que debemos incorporarla como variable en nuestros estudios, de tal manera que incorporamos las preferencias sociales a la toma de decisiones.

8. Los programas asistenciales de atención farmacéutica y nuevos servicios clínicos se verán reforzados al aplicar esta tecnología para su implantación por demostrar su valor. 
BIBLIOGRAFÍA 
Bibliografía

Aaronson NK, Ahmedzai S, Bergman B, Bullinger M, Cull A, Duez NJ, Filiberti A, Flechtner H, Fleishman SB, de Haes JC, et al (1993). The European Organization for Research and Treatment of Cancer QLQ-C30: a quality-of-life instrument for use in international clinical trials in oncology. $\mathrm{J}$ Natl Cancer Inst 85(5): 365-76.

Akehurst R, Anderson P, Brazier J, Brennan A, Briggs A, Buxton M (2000). Decision analytic modelling in the economic evaluation of health technologies-a consensus statement. Pharmacoeconomics 17: 443-4.

Amat Díaz M, Poveda Andrés JL, Carrera-Hueso FJ (2011). Aplicación de la farmacoeconomía en la gestión clínica. Farm Hosp 35 (Supl 1): 18-24.

Anderson SV, Schumock GT (2009). Evaluation and justification of clinical pharmacy services. Expert Rev Pharmacoeco Outcomes Res 9(6): 539-45.

Auray J-P, Béresniak A, Claveranne J-P, Duru G, Murillo C (1998). Diccionario comentado de economía de la salud. Barcelona: Masson. p. 221.

Bauer KA, Eriksson BI, Lassen MR, Turpie AG; Steering Committee of the Pentasaccharide in Major Knee Surgery Study (2001). Fondaparinux compared with enoxaparin for the prevention of venous thromboembolism after elective major knee surgery. N Engl J Med 345(18): 1305-1310.

Bergner M, Bobbitt RA, Pollard WE, Martin DP, Gilson BS (1976). The sickness impact profile: validation of a health status measure. Med Care 14(1): 57-67.

Bernal Delgado E, Ortún-Rubio V (2010). La calidad del Sistema Nacional de Salud: base de su deseabilidad y sostenibilidad. Gac Sanit 24 (3): 254-258.

Bootman JL, McGhan WF, Townsend RJ, eds (2004). Principles of Pharmacoeconomics Third edition. Houston, Texas: Harvey Whitney Books Company. 
Brosa M, Gisbert R, Rodríguez JM, Soto J (2005). Principios, métodos y aplicaciones del análisis del impacto presupuestario en el sector sanitario. Pharmacoeconomics-Spanish Res 2(2): 64-78.

Bungay KM, Boyer JG, Ware JE (2005). Health-related quality of life: an overview. In: Bootman JL, Towsend RJ, McGhan WF, eds. Principles of pharmacoeconomics $3^{a}$ Edition. Cincinnati: Harvey Whiyney Books Company, 149-173.

Calleja MA, Faus Dader MJ, Tuneu L, Silva MM (2008). Seguimiento farmacoterapéutico en pacientes hospitalarios. Granada: Universidad de Granada.

Campbell A (1981). The sense of well-being in America: recent patterns and trenes. New York: McGraw-Hill.

Camps C, Caballero C, Sirera R, Blasco A, Cayuela D, Gil M,-Badía X (2008). Can the Spanish care system assume the new costs of medications against cancer? Clin Transl Oncol 10: 96-101.

Camps C, Iranzo V,-Caballero C,-Blasco A, Godes MJ,-Safont MJ,-Blasco S, - Mengual G,-Berrocal A,-Sirera R (2011). Oncology outside hospital: a new experience for the benefi t of longer survivors. Clin Transl Oncol 13(4):249-53.

Carrera-Hueso FJ, Giráldez J (1995). Estructuras de soporte en la toma de decisiones farmacoterapéuticas: El farmacéutico. Farm Hosp 19 (3): 169-173.

Carrera-Hueso FJ, Ronchera-Oms CL (1996). Estrategias para el manejo de estudios farmacoeconómicos. NFT 15: 9-12.

Carrera-Hueso FJ (1997). Análisis de los resultados sanitarios y su aplicación. Rev Med Univ Navarra 41: 31-38. 
Carrera-Hueso FJ (1997a). Estudios de calidad de vida relacionados con la salud. Farm Clín 14: 98-109.

Carrera-Hueso FJ (1997b). Calidad de vida en un paciente epiléptico. Farm Clín 14: 74-89.

Carrera-Hueso FJ (1998). Aplicación de los estudios farmacoeconómicos en el hospital. Med Clín (Barc) 111: 347-53.

Carrera-Hueso FJ (2000). Aplicación de los estudios farmacoeconómicos y de calidad de vida relacionada con la salud. Rev Esp Farmacoeconomía III: 17-33.

Carrera-Hueso FJ, Poquet Jornet JE, Ramón Barrios A, Conde Farnández F (2010). Coste-efectividad en la inducción del parto con dinoprostona. Aten Farm 12 (6): 359-68.

Carrera-Hueso FJ, Ramón Barrios A, Carrera Hueso JA, Poquet Jornet JE (2010a). Coste-efectividad de la tromboprofilaxis extendida en fractura de cadera: fondaparinux frente a enoxaparina. Aten Farm 12 (3): 144-55.

Carrera-Hueso FJ (2011). El valor de la farmacoeconomía. Farm Hosp 35 (Supl 1): $1-2$.

Carrera-Hueso FJ, Ramón Barrios A (2011). Análisis de sensibilidad estructural. Farm Hosp 35 (Supl 1): 10-17.

Cella DF, Tulsky DS, Gray G, Sarafian B, Linn E, Bonomi A, Silberman M, Yellen SB, Winicour P, Brannon J, et al (1993). The Functional Assessment of Cancer Therapy scale: development and validation of the general measure. J Clin Oncol 11(3): 570-9.

Claxton K, Sculpher M, McCabe C, Briggs A, Akehurst R, Buxton M, Brazier J, O'Hagan T (2005). Health Econ 14: 339-347. 
Cosialls Pueyo D (2000). Gestión clínica. Rev Adm San 16:119-139.

Darbá J (2006). La utilización de métodos probabiblísticos para la evaluación económica de tecnologías sanitarias. Gac Sanit 20(1): 74-9.

DATA $®$ v 3.5 (2011). TreeAge Software Inc. Disponible en internet: http://www.treeage.com/index.htm [Consulta: 20 de enero 2011].

de Haes JC, Stiggelbout AM (1996). Assessment of values, utilities and preferences in cancer patients. Cancer Treat Rev 22 Suppl A: 13-26.

De Rijdt T, Willems L, Simoens S (2008). Economic effects of clinical pharmacy interventions: a literature review. Am J Health Syst Pharm 65(12): 1161-72.

Del Llano J, Ruiz J, Pi G (2008). Del concepto de la utilidad terapéutica de los medicamentos a su aplicabilidad. Madrid: Fundación Gaspar Casal. GSK.

Delgado O (2007). Conciliación de la medicación. Med Clin (Barc) 129(9): 343-8

Delgado O, Nicolás J, Martínez I, Serrano A, Anoz L Fernández F (2009). Errores de conciliación en el ingreso y en el alta hospitalaria en pacientes ancianos polimedicados. Estudio prospectivo aleatorizado multicéntrico. Med Clin (Barc) 133(19):741-744

Delgado O, Puigventós F, Clopés A (2009a). Posicionamiento del farmacéutico de hospital ante la utilización de medicamentos en condiciones diferentes a las autorizadas. Farm Hosp 33(5): 237-9

Devinsky O, Vickrey BG, Cramer J, Perrine K, Hermann B, Meador K, Hays RD (1995). Development of the quality of life in epilepsy inventory. Epilepsia 36(11): 1089-104.

Díez Vegas FJ (2005). Probabilidad y teoría de la decisión en medicina. Herramientas de Gestión e investigación sanitaria. Madrid: UNED. 
DiMasi JA, Caglarcan E, Wood-Armany M (2001). Emerging role of Pharmacoeconomics in the research and development decision-making process. Pharmacoeconomics 19 (7): 753-66.

Donabedian A (1988). The quality of care: how can it be assessed?. JAMA 260; 1743-48.

Drummond MF, Stoddart GL, Torrance GW, Sculpher MJ, O'brien BJ, eds (2005). Methods for the Economic Evaluation of Health Care Programmes. Oxford: Oxford Univ Press.

Ellwood PM (1988). Outcomes management: a technology of patient experience. N Engl J Med 318; 1549-56.

Elvira ${ }^{\circledR}$ versión $0.162 \quad$ (2010). UNED. Disponible en internet: http://www.ia.uned.es/ elvira/instalar/instalar.html [Consulta: 16 de febrero de 2010]

Eriksson $\mathrm{BI}$, Lassen MR; PENTasaccharide in HIp-FRActure Surgery Plus Investigators (2003). Duration of prophylaxis against venous thromboembolism with fondaparinux after hip fracture surgery: a multicenter, randomized, placebocontrolled, double-blind study. Arch Intern Med 163(11): 1337-42.

Espín J, Oliva J, Rodríguez-Barrios JM (2010). Esquemas innovadores de mejora del acceso al mercado de nuevas tecnologías: los acuerdos de riesgo compartido. Gac Sanit 24(6): 491-497.

EuroQol Group (1990). EuroQol--a new facility for the measurement of healthrelated quality of life. Health Policy 16(3): 199-208.

Fayers PM, Machin D, eds (2007). Quality of Life: the assessment, analysis and interpretation of patient-reported outcomes, 2nd ed. Chichester (UK): John Wiley \& son. 
García Armesto S, Abadía B, Durán A, Bernal E (2010). Health Systems in transition: España. Análisis del sistema sanitario 2010 [monografía en internet]. Sociedad Española de Salud pública y Administración Sanitaria. Disponible en internet: http://www.sespas.es/adminweb/uploads/docs/HiT2010.pdf. [Consulta: 10 de enero 2011].

GENESIS (2011). Grupo de Evaluación de Novedades, Estandarización e Investigación en Selección de Medicamentos. Sociedad Española de Farmacia Hospitalaria. Disponible en internet: http://genesis.sefh.es/. [Consulta: 10 abril 2011].

Gimeno JA, Rubio S, Tamayo P, eds (2006). Economía de la salud: instrumentos. Manuales de Dirección Médica y Gestión Clínica. Madrid: Ediciones Díaz de Santos.

Haentjens P, De Gropte K, Annemans L (2004). Prolonged enoxaparin therapy to prevent venous thromboembolism after primary hip or knee replacement. A cost-utility analysis. Arch Orthop Trauma Surg 124 (8): 507-17.

Hepler CD, Strand LM (1990). Opportunities and responsibilities in pharmaceutical care. Am J Health Syst Pharm 47: 533-543.

Hickey AM, Bury G, O'Boyle CA, Bradley F, O'Kelly FD, Shannon W (1996). A new short form individual quality of life measure (SEIQoL-DW): application in a cohort of individuals with HIVIAIDS. BMJ 313(7048): 29-33.

Hunt SM, McEwen J, McKenna SP (1985). Measuring health status: a new tool for clinicians and epidemiologists. J R Coll Gen Pract 35(273): 185-8.

Johnson JA, Bootman JL (1995). Drug-related morbility and mortality. A cost-ofillness model. Arch Intern Med 155: 1949-56. 
Juniper EF, Guyatt GH, Feeny DH, Ferrie PJ, Griffith LE, Townsend M (1996). Measuring quality of life in children with asthma. Qual Life Res 5(1): 35-46.

Khan ZM, Miller DW (1999). Modeling economic evaluation of pharmaceuticals: manipulation or valuable tool? Clin Ther 21: 896-908.

Kopp BJ, Mrsan M, Erstad BL, Duby JJ (2007). Cost implications of and potential adverse events prevented by interventions of a critical care pharmacist. Am J Health Syst Pharm 64(23): 2483-7.

Kozma CM, Reeder CE, Schultz RM (1993). Economic, clinical, and humanistic outcomes: a planing model for pharmacoeconomic research. Clin Ther 15: 112132.

Kuntz KM, Weinstein MC (2001). Modelling in economic evaluation. In Drummond $\mathrm{M}$ and McGuire $\mathrm{A}$. Economic Evaluation in Health Care, merging the theory with practice. Oxford, UK. OHE Oxford University Press.

Lang DL, Lopert R, Hill SR (2003). Use of pharmacoeconomic in prescribing research. Part 5: modelling beyond clinical trials. Journal of Clinical Pharmacy and Therapeutics 28: 433-9.

Lassen MR, Bauer KA, Eriksson BI, Turpie AG; European Pentasaccharide Elective Surgery Study (EPHESUS) Steering Committee (2002). Postoperative fondaparinux versus preoperative enoxaparin for prevention of venous thromboembolism in elective hip-replacement surgery: a randomised doubleblind comparison. Lancet 359: 1715-20.

LEY 29/2006, de 26 de julio, de garantías y uso racional de los medicamentos y productos sanitarios. BOE núm. 128, pág: 28122-28165, de 29 mayo 2003. 
López-Bastida J, Oliva J, Antoñanzas F, García-Altés A, Gisbert R, Mar J, PuigJunoy J (2010). Propuesta de guía para la evaluación económica aplicada a las tecnologías sanitarias. Gac Sanit 24(2): 154-170.

López González AM, Sánchez Sánchez T (2007). La gestión del conocimiento como herramienta clave para la mejora de las organizaciones sanitarias. Farm Hosp 31: 3-4.

Lorenzo S (2006). Busque, compare, y si encuentra algo mejor...Farm Hosp 30: 65-67.

Lundkvist J, Bergqvist D, Jönsson B (2007). Cost-effectiveness of extended prophylaxis with fondaparinux compared with low molecular weight heparin against venous thromboembolism in patients undergoing hip fracture surgery. Eur J Health Econ 8(4): 313-23.

IMS (2010) Indicadores del mercado hospitalario. IMS-España. Disponible en internet:

http://www.aimfa.es/Varios/ftp/Indicadores\%20Mercado\%20Hospitalario\%20julio \%202010.pdf [Consulta: 16 de abril 2011].

Inglehart JK (1994). Health policy report: phycisians and the growth of manage care. N Engl J Med 331; 1167-71.

ISPOR Task Force (2003). Principles of good practice for decision analytic modeling in health-care evaluation. Value Health 6 (1): 9-17.

Mahoney FI, Barthel DW (1965). Functional evaluation: the barthel index. Md State Med J 14: 61-5.

Mar J, Sainz-Ezkerra M, Miranda-Serrano E (2008). Calculation of prevalence with markov models: budget impact analysis of thrombolysis for stroke. Med Decis Making : 28(4): 481-90. 
Martín-Conde JA, Tévar Alfonso E, García García FJ (2011). ¿Valen los medicamentos lo que cuestan? Farm Hosp 35 (Suppl 1), 32-39.

Melzack R (1975). The McGill Pain Questionnaire: major properties and scoring methods. Pain 1(3): 277-99.

Monleón T, Ocaña J (2006). La simulación como herramienta de optimización de la investigación clínica. Med Clin (Barc) 127: 702-4.

MSPSI (2006). Encuesta Nacional de Salud de España 2006. Madrid. Instituto Nacional de Estadística. Disponible en internet: http://www.mspsi.es/estadEstudios/estadisticas/encuestaNacional/encuestalndic e2006.htm [Consulta: 16 de abril 2011].

MSPSI (2010). Datos de facturación de Receta Médica 2010. Madrid. Instituto de Información Sanitaria. Disponible en internet: http://www.msc.es/profesionales/farmacia/datos/diciembre2010.htm [Consulta: 16 de abril 2011].

Nuitjen M, Rutten F (2000). Combining a budget impact analysis and costeffectiveness analysis using decision analytic modelling technique. Pharmacoeconomics 20(22): 855-57.

OCDE (2010). Organisation for Economic Co-operation and Developement. Health Data 2010: key indicators. Disponible en internet: http://www.irdes.fr/EcoSante/DownLoad/OECDHealthData_FrequentlyRequeste dData.xls. [Consulta: 12 de enero 2011].

Oliva J, Antoñanzas F, Rivero-Arias O (2008). Evaluación económica y la toma de decisiones en salud. El papel de la evaluación económica en la adopción y difusión de tecnologías sanitarias. Informe SESPAS2008. Gac Sanit 1:137-42. 
Oliva J, Bernal E, Puig-Junoy J (2008a). Evaluación económica de medicamentos: experiencias y vías de avance. Una visión complementaria. Gac Sanit 22: 358-61.

Ortún V, editor (2003). Gestión clínica y sanitaria: De la práctica diaria a la academia, ida y vuelta. Barcelona: Masson.

Otero López MJ (2010). El papel del farmacéutico en la gestión de la seguridad de los medicamentos diez años después de la publicación del informe "Errar es humano". Farm Hosp 34: 159-62.

Pardo López MÁ, Aznar Saliente MT, Soler Company E (2008). CONSULTENOS: programa de información al alta hospitalaria. Desarrollo y resultados del primer año de funcionamiento en 5 hospitales. Farm Hosp 32(6): 323-30.

Patrick DL, Ericsson P (1993). Health status and health policy. Allocating resources to health care. New York: Oxford University Press.

Peiró S (2006). Atención farmacéutica en el entorno hospitalario: la hora de las decisiones. Farm Hosp 30: 325-27.

Peiró S, Artells JJ, Meneu R (2011). Identificación y priorización de actuaciones de mejora de la eficiencia en el Sistema Nacional de Salud. Gac Sanit 25(2): 95105.

Pinteño M, Martínez-López I, Delgado O (2006). Equivalentes terapéuticos: Concepto y niveles de evidencia. El Farmacéutico Hospitales 178: 5-10.

Pinto JL, Abellán JM (2008). Farmacoeconomía: el gran reto de la sanidad pública. Humanidades Médicas 32: 11-21. 
Pinto JL, Sánchez FI, Abellán JM, eds (2004). Incorporación de las preferencias de los pacientes en la toma de decisiones clínicas. Barcelona: Masson.

Pinto-Prades JL, Loomes G, Brey R (2009). Trying to estimate a monetary value for the QALY. Journal of Health Economics 28: 553-62.

Poquet Jornet JE, Carrera-Hueso FJ, Gasent Blesa JM, Peris Godoy M (2011). Aspectos farmacoeconómicos de los citostáticos orales. Farm Hosp 35 (Supl 1): 25-31.

Puig-Junoy J, Meneu R (2005). Aplicación de los contratos de riesgo compartido a la financiación de nuevos medicamentos. Gestión Clínica y Sanitaria 7(3): 8894.

Puig-Junoy J, Pinto JL (2008). Los métodos de evaluación económica de medicamentos, tecnologías médicas y programas de salud. Programa de Formación en Farmacoeconomía y Economía de la Salud. Universitat Pompeu Fabra, CRES-UPF. Barcelona.

Puigventós F, Santos-Ramos B, Ortega A, Durán-García E (2010). Structure and procedures of the pharmacy and therapeutic committees in Spanish hospitals. Pharm World Sci 32(6): 767-75.

Raferty J (2000). Economic notes: Costing in economic evaluation. Br Med J 318: 1551-2.

Real Decreto-ley 4/2010, de 26 de marzo, de racionalización del gasto farmacéutico con cargo al Sistema Nacional de Salud. BOE núm. 75: pág. 28989-29000, de 27 de marzo de 2010.

Real Decreto-ley 8/2010, de 20 de mayo, por el que se adoptan medidas extraordinarias para la reducción del déficit público. BOE núm. 126: pág. 4507045128, de 24 de mayo de 2010. 
Relman AS (1988). Assessment and accountability: The third era revolution in medical care. N Engl J Med 319; 1220-21.

Relman AS (2009). Doctors as the key to health care reform. N Engl J Med 361(13); 1225-7.

Repullo Labrador JR (2006). Cambios y reformas en sistemas y servicios sanitarios. En: Gimeno JA, Rubio S, Tamayo P, eds. Sistemas y servicios sanitarios. Manuales de Dirección Médica y Gestión Clínica. Madrid: Ediciones Díaz de Santos: 121-97.

Ríos Insua S, Bielza Lozoya C, Mateos Caballero A (2002). Fundamentos de los sistemas de ayuda a la decisión. Madrid: Editorial RA-MA.

Robert M, Stephen G, Pauker M (1983). The Markov Process in medical Prognosis. Med Decis Making 3: 419-45.

Rodríguez Artalejo F, Banegas Banegas JR, González Enríquez J, Martín Moreno JM, Villar Álvarez F (1990). Análisis de decisiones clínicas. Med Clin (Barc) 94: 348-54.

Rodríguez-Barrios JM (2004). Papel de los modelos en las evaluaciones económicas en el campo sanitario. Farm Hosp 28 (4): 231-42.

Rodríguez-Barrios JM, Serrano D, Monleón T, Caro J (2008). Los modelos de simulación de eventos discretos en la evaluación económica de tecnologías y productos sanitarios. Gac Sanit 22(2): 151-61.

Rubio-Terrés C (2000). Introducción a la utilización de los modelos de Markov en el análisis farmacoeconómico. Farm Hosp 24 (4): 241-7. 
Rubio-Terrés C, Cobo E, Sacristán JA, Prieto L, del Llano J, Badia X (2004). Análisis de la incertidumbre en las evaluaciones económicas de intervenciones sanitarias. Med Clin (Barc) 122(17): 668-74.

Sacristán JA, Badía X, Rovira J, eds (1995). Farmacoeconomía: Evaluación económica de medicamentos. Madrid: Editores médicos SA.

Sanchez LA (1996). Pharmacoeconomics and formulary decision making. Pharmacoeconomics 9 (Suppl 1): 16-25.

SEGO (2004). Protocolos asistenciales en Obstetricia. Embarazo cronológicamente prolongado. Protocolo de la S.E.G.O. Madrid.

Segura A (2010). La participación ciudadana, la sanidad y la salud. Gestión clínica y sanitaria 12 (2): 55-62.

Siebert U (2003). When should decision-analytic modeling be used in the economic evaluations of heath care? Eur J Health Econom 4: 143-50.

Silva-Castro MM, Tuneu I, Valls L, Faus MJ (2010). Revisión sistemática sobre la implantación y la evaluación del seguimiento farmacoterapéutico en pacientes hospitalizados. Farm Hosp 34(3): 106-124.

Slof J, Badia X (2006). Evaluación clínica y económica de la profilaxis con fondaparinux en comparación con enoxaparina en cirugía ortopédica mayor. Pharmacoeconomics-Spanish Research Articles 3 (2): 75-88.

Smets EM, Garssen B, Bonke B, De Haes JC (1995). The Multidimensional Fatigue Inventory (MFI) psychometric qualities of an instrument to assess fatigue. J Psychosom Res 39(3): 315-25. 
Soto J (2002). Health economic evaluations using decision analytic modelling. Principles and practices-utilization of a checklist to their development and appraisal. Int J Technol Assess Health Care 18: 94-111.

Stahl JE (2008). Modelling methods for pharmacoeconomics and health technology assessment: an overview and guide. Pharmacoeconomics 26(2): 131-48.

Taylor S, Armour C (2003). Consumer preference for dinoprostone vaginal gel using stated preference discrete choice modelling. Pharmacoeconomics 21(10): 721-35.

Townsend RJ (1987). Postmarketing drug research and development. Drug Intell Clin Pharm 21(1 Pt 2):134-6.

Trueman P, Drummond M, Hutton J (2001). Developing guidance for budget impact analysis. Pharmacoeconomics 19(6): 609-21.

Turpie AG, Bauer KA, Eriksson BI, Lassen MR; PENTATHALON 2000 Study Steering Committee (2002). Postoperative fondaparinux versus postoperative enoxaparin for prevention of venous thromboembolism after elective hipreplacement surgery: a randomised double-blind trial. Lancet 359: 1721-6.

Viladrich C, Doval E (2011). Medición: Fiabilidad y validez (5a ed.). Bellaterra: Laboratori d'Estadística Aplicada i de Modelització (UAB).

Wade WE, Spruill WJ, Leslie RB (2004). Cost analysis of fondaparinux versus enoxaparin as venous thromboembolism prophylaxis in hip Venous Thromboembolism prophylaxis fracture surgery. Am J Ther 11(3): 194-8.

Walters SJ, ed (2009). Quality of life outcomes in clinical trials and health-care evaluation: a practical guide to analysis and interpretation. Chichester (UK): John Wiley \& son. 
Ware JE (1995). The status of health assessment 1994. Ann Rev Public Health 16: $327-54$

Ware JE Jr, Sherbourne CD (1992). The MOS 36-Item Short-Form Health Survey (SF-36). Med Care 32: 473-482.

Weinstein MC (1980) Cost-effectiveness analysis for clinical procedures in oncology. Bull Cancer 67(5): 491-500.

WHO (1948). Basic documents: World Health Organization. Geneve, Switzerland: World Health Organization.

Willan AR, Briggs AH, eds (2006). Statistical analysis of cost-effectiveness data. West Sussex (England): John Wiley \& Sons Ltd.

Wing DA, Rahall A, Jones MM, Goodwin TM, Paul RH (1995). Misoprostol: an effective agent for cervical ripening and labor induction. Am J Obstet Gynecol 172(6): 1811-6.

Zigmond AS, Snaith RP (1983). The hospital anxiety and depression scale. Acta Psychiatr Scand 67(6): 361-70. 